\title{
Total Synthesis of (-)-Maximiscin
}

Kyle S. McClymont, Feng-yuan Wang, Amin Minakar, Phil S. Baran.

Department of Chemistry, The Scripps Research Institute (TSRI), 10550 North Torrey Pines

Road, La Jolla, California, 92037, USA

\section{Supporting Information}




\section{Table of Contents}

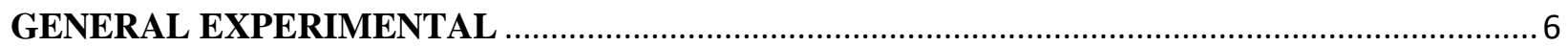

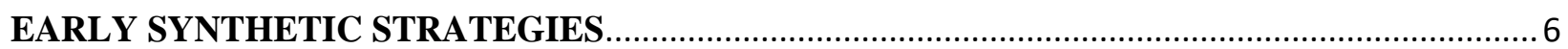

Decarboxylative Cross Coupling Disconnection ....................................................................... 6

Route to Carboxylic Acid Coupling Partner ........................................................................ 7

Decarboxylative Cross Coupling Optimization ..................................................................... 7

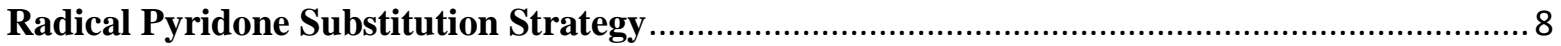

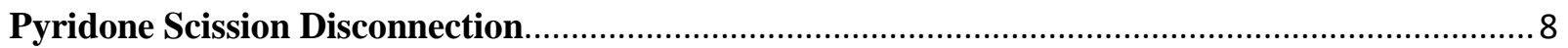

C-H Activation via 7-Membered Metallacycle ..................................................................... 9

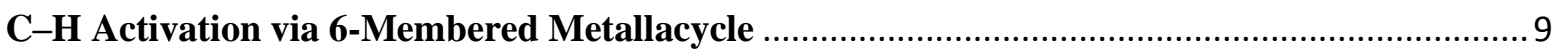

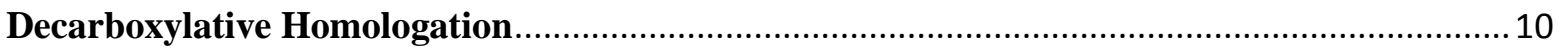

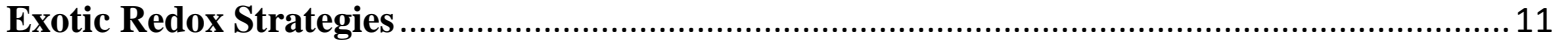

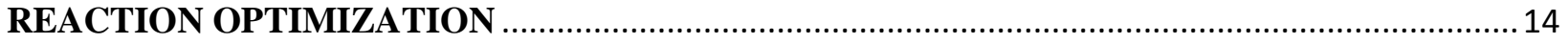

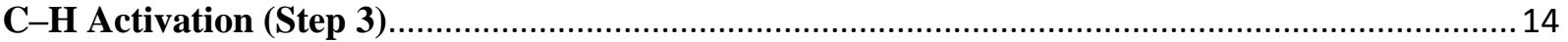

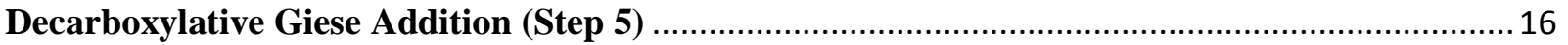

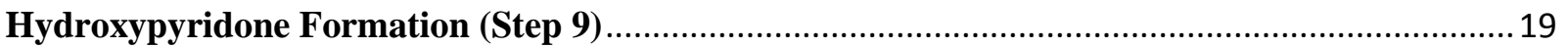

SYNTHETIC PROCEDURES AND CHARACTERIZATION DATA ............................................24

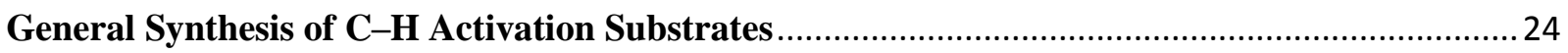

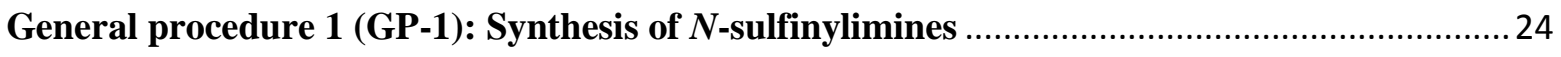

General procedure 2 (GP-2): Synthesis of $N$-sulfinamides …............................................. 24

General procedure 3 (GP-3): Synthesis of amine dihydrochlorides........................................ 24

General procedure 4 (GP-4): Synthesis of C-H activation substrates ....................................2 25

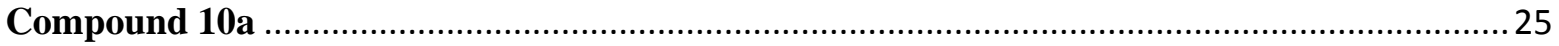

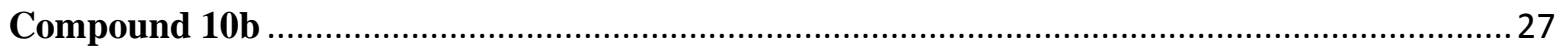

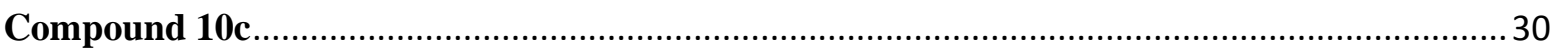

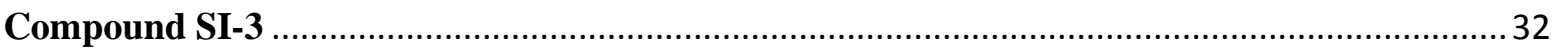

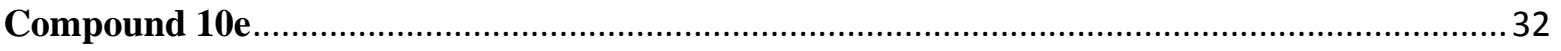

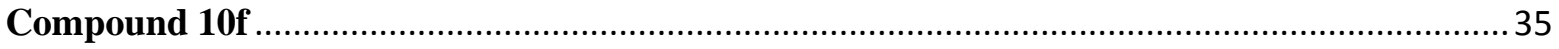

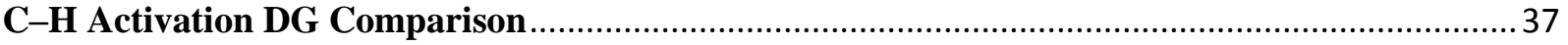

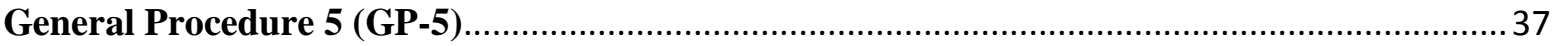

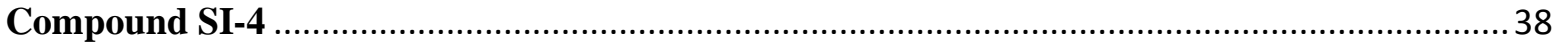

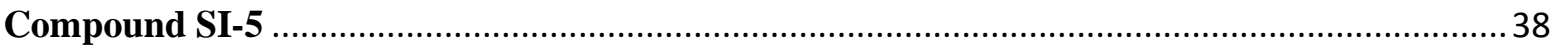

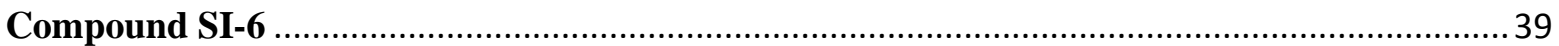




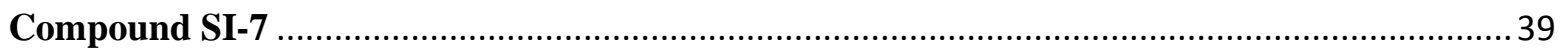

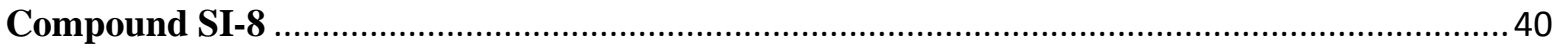

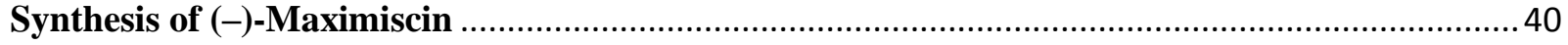

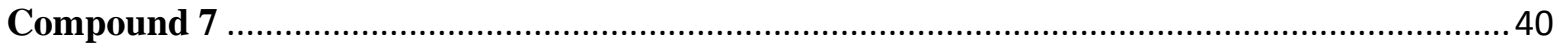

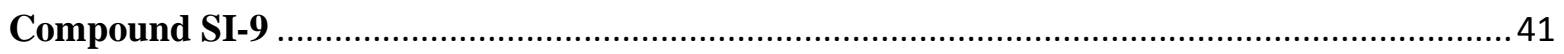

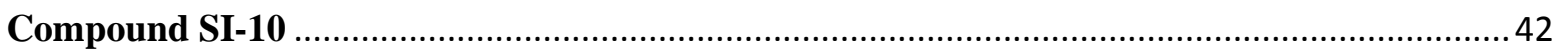

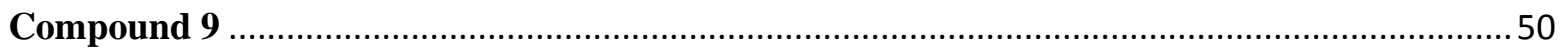

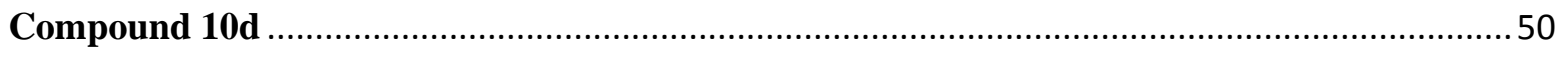

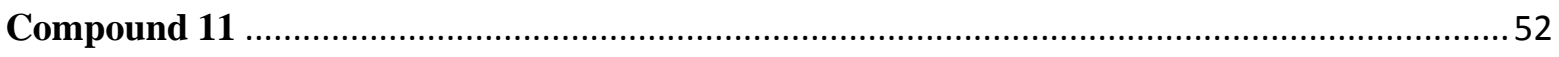

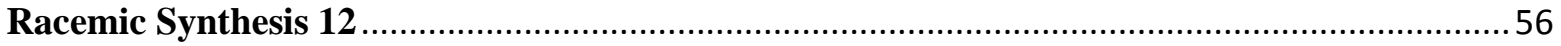

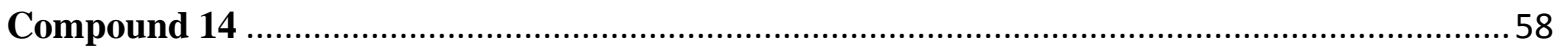

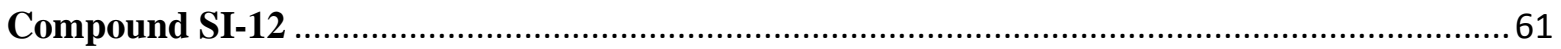

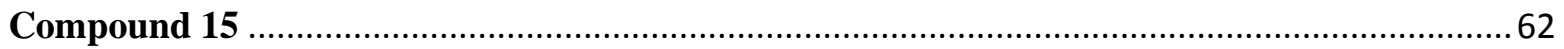

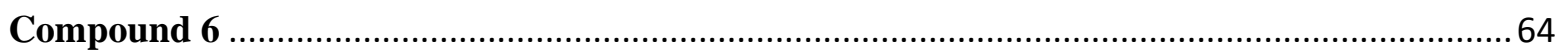

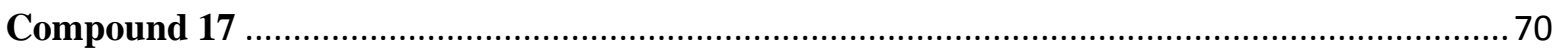

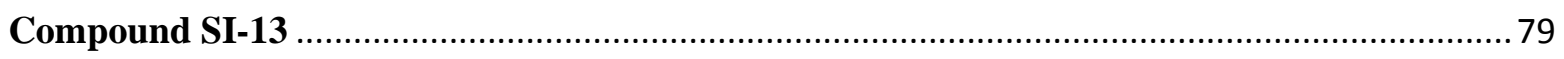

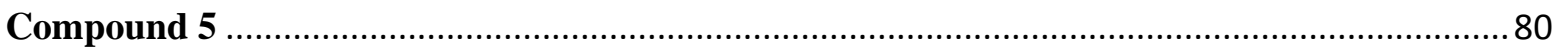

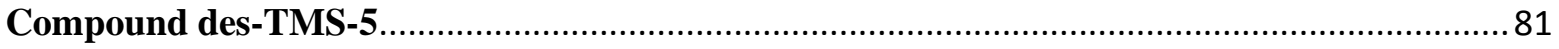

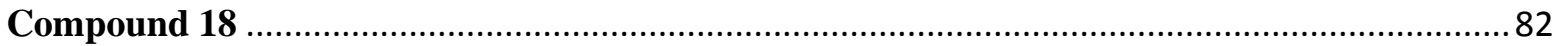

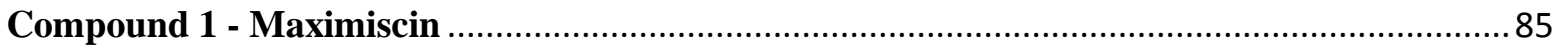

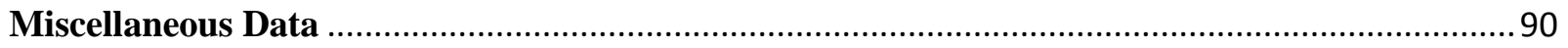

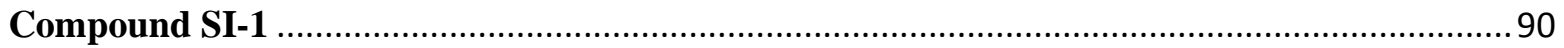

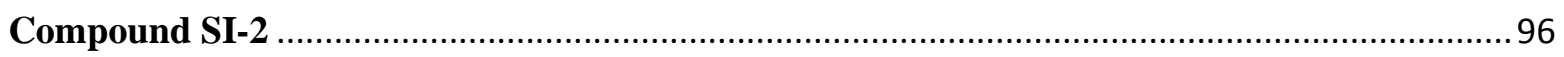

C-H Activation Product Inhibition Study ........................................................................ 96

REFERENCES

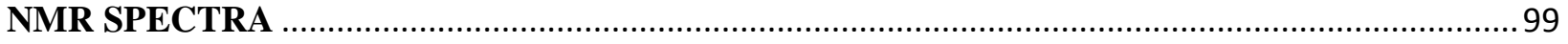

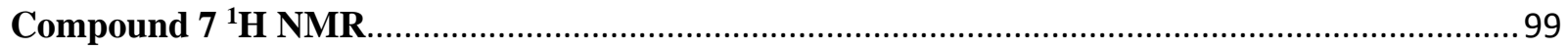

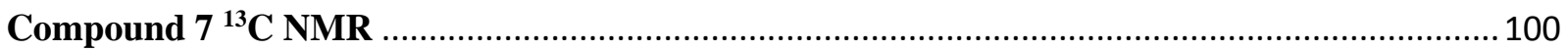

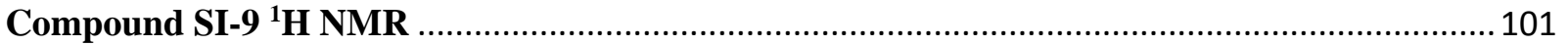

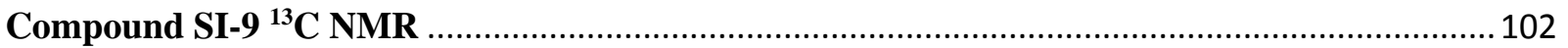

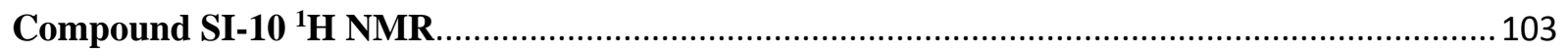

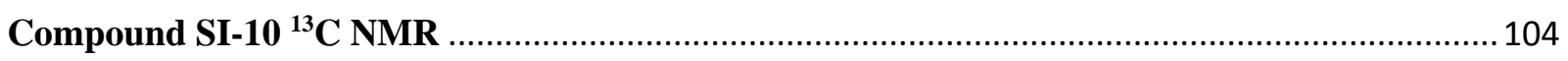

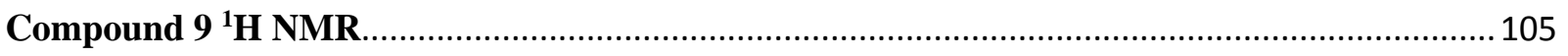




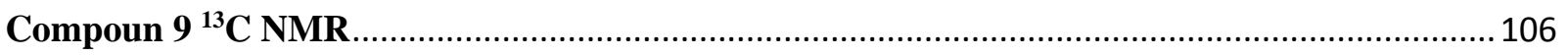

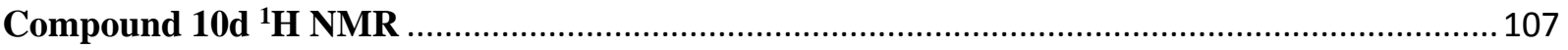

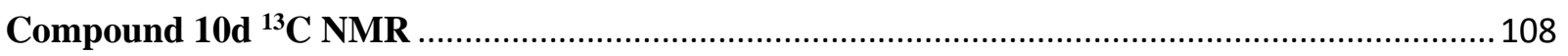

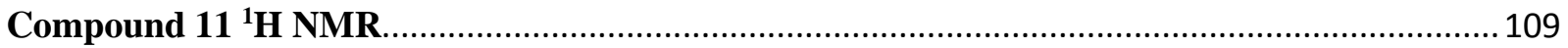

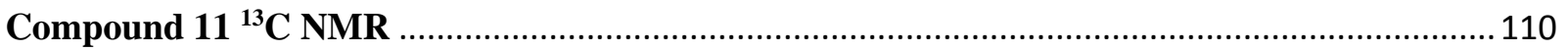

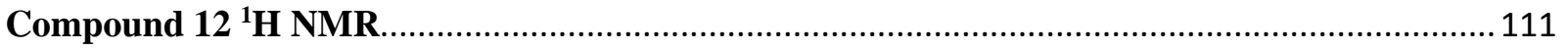

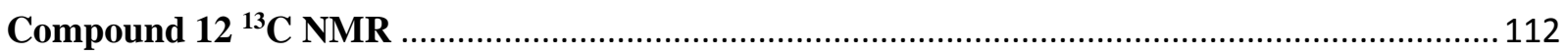

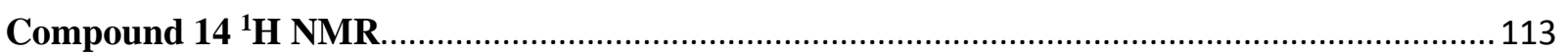

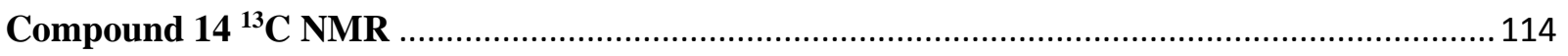

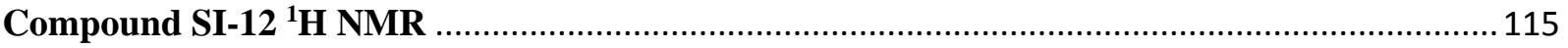

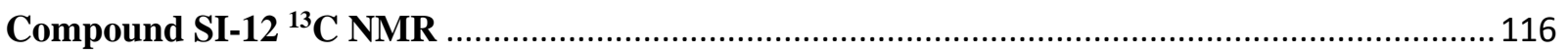

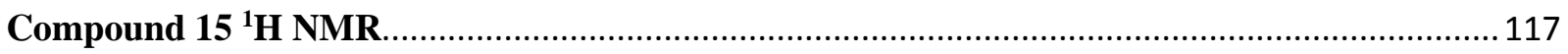

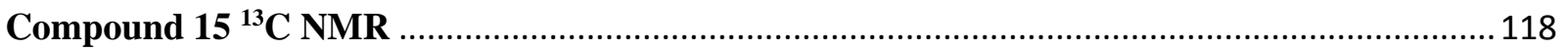

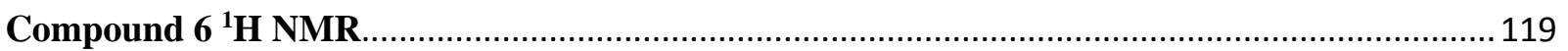

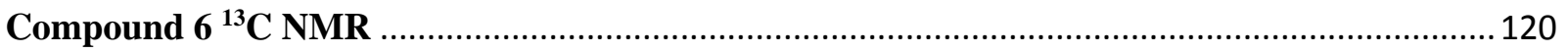

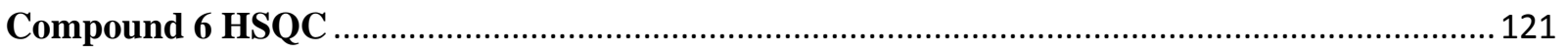

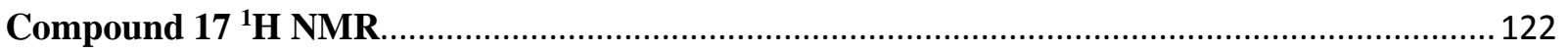

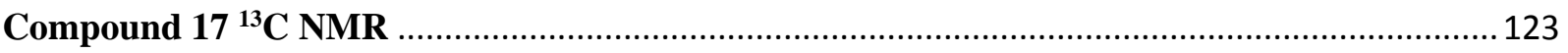

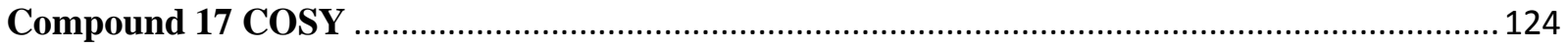

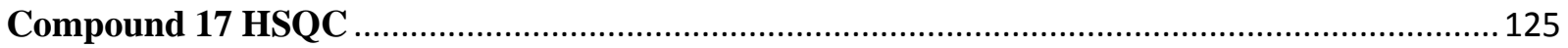

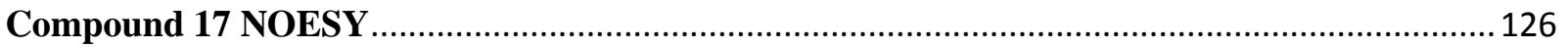

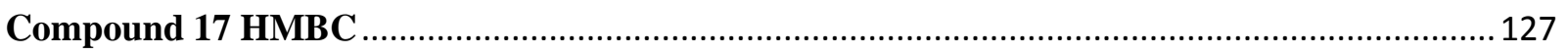

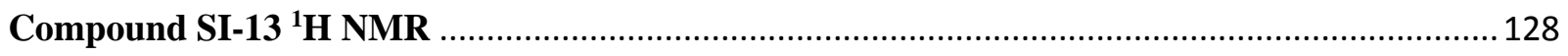

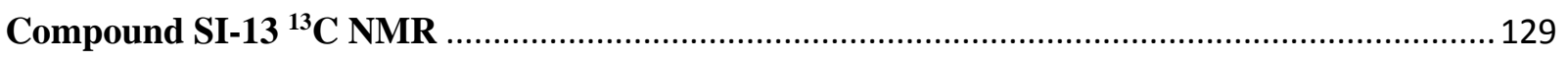

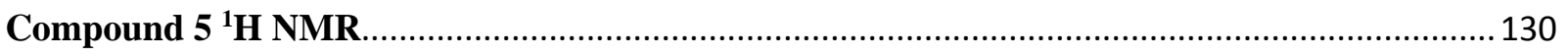

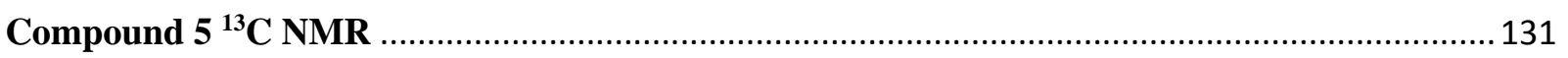

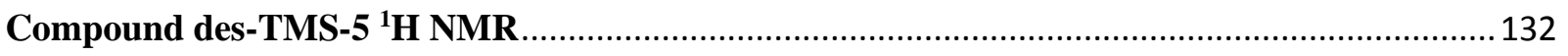

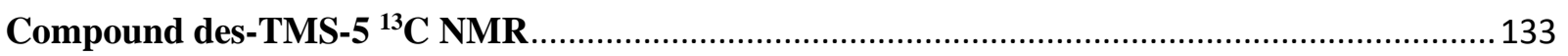

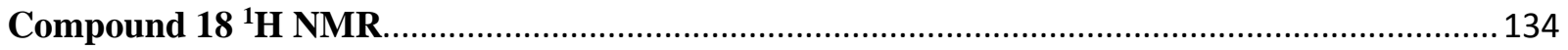

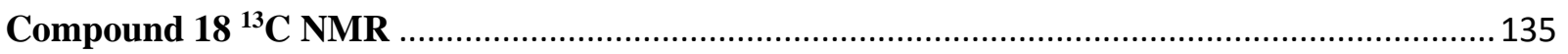

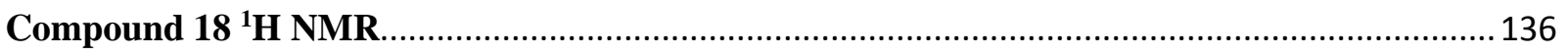

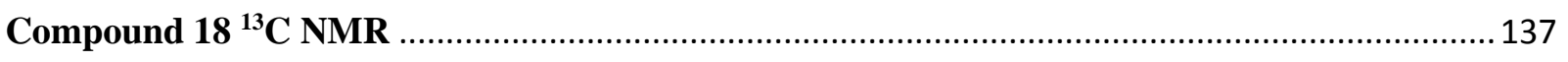

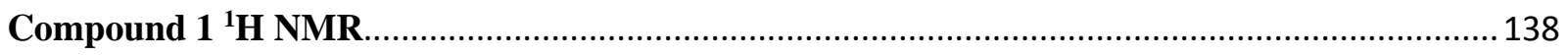




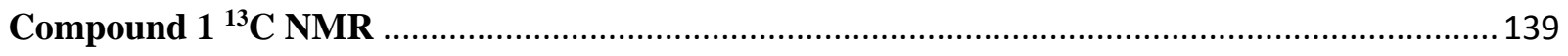

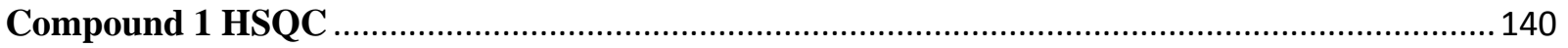

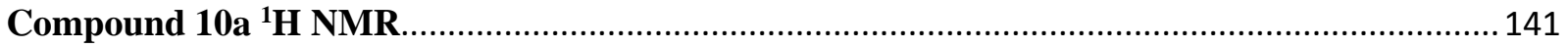

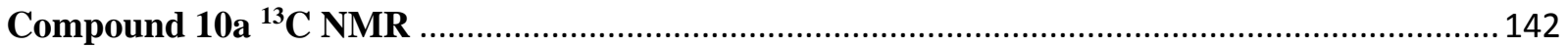

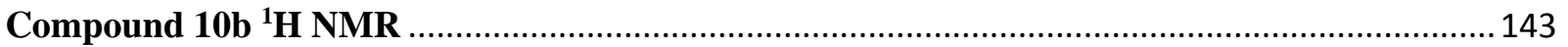

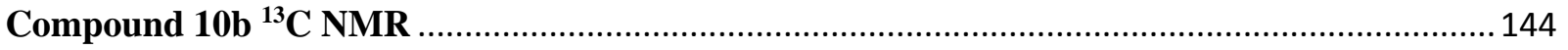

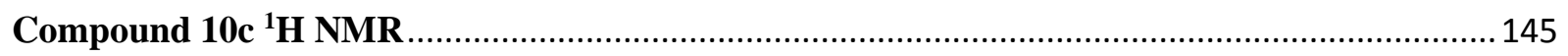

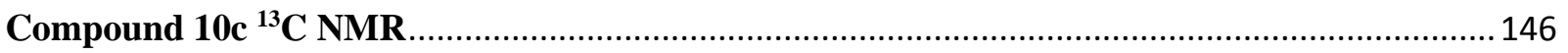

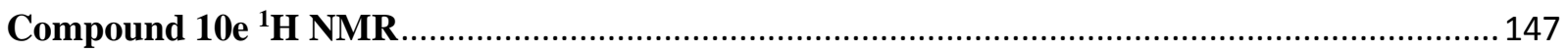

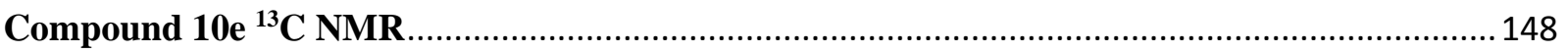

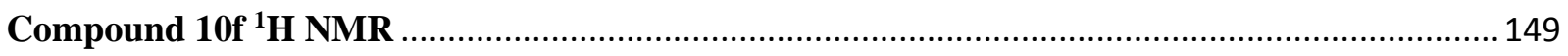

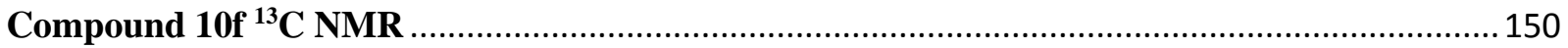

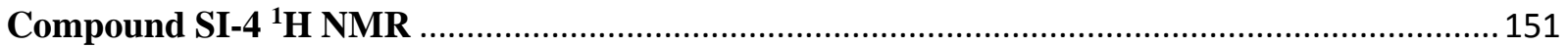

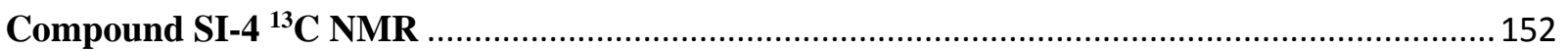

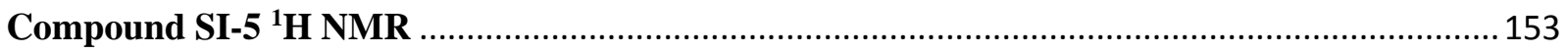

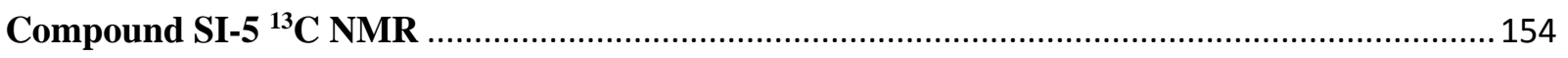

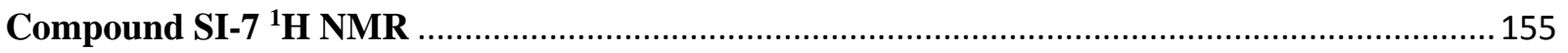

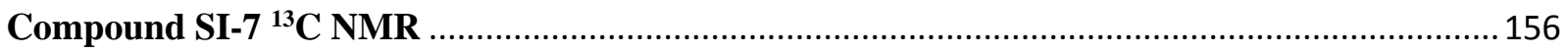

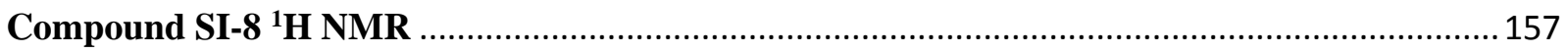

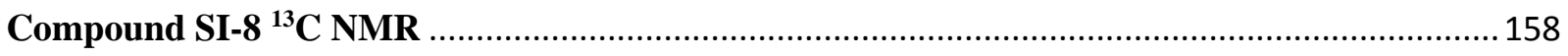

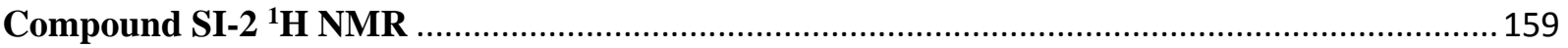

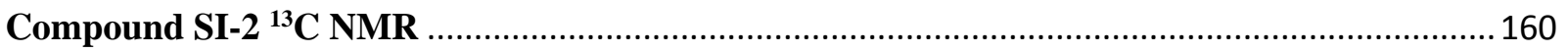

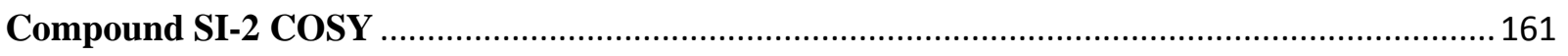

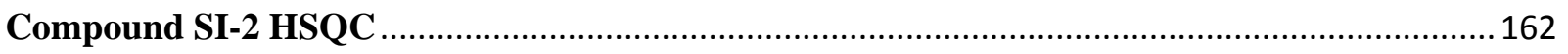




\section{GENERAL EXPERIMENTAL}

Tetrahydrofuran (THF), diethyl ether $\left(\mathrm{Et}_{2} \mathrm{O}\right)$, dichloromethane $\left(\mathrm{CH}_{2} \mathrm{Cl}_{2}\right)$, toluene, $N, N$ dimethylformamide (DMF), acetonitrile, methanol $(\mathrm{MeOH})$ and diisopropylamine (DIPA) were obtained by passing the previously degassed solvents through an activated alumina column. Reagents were purchased at the highest commercial quality and used without further purification, unless otherwise stated. $\mathrm{Pd}(\mathrm{OAc})_{2}$ was purchased from Strem. $\mathrm{PtO}_{2}, \mathrm{AgNO}_{3}$ and $\mathrm{Fe}_{2}\left(\mathrm{SO}_{4}\right)_{3} \cdot 5 \mathrm{H}_{2} \mathrm{O}$ were purchased from SigmaAldrich. AgOTf was purchased from Strem and was stored and weighed in a glove box. Yields refer to chromatographically and spectroscopically $\left({ }^{1} \mathrm{H}\right.$ NMR) homogeneous material, unless otherwise stated. Reactions were monitored by GC/MS, LC/MS, and thin layer chromatography (TLC). TLC was performed using $0.25 \mathrm{~mm}$ E. Merck silica plates (60F-254), using short-wave UV light as the visualizing agent, and $\mathrm{KMnO} 4$ with heat as developing agent. NMR spectra were recorded on Bruker DRX-600, DRX-500, and AMX-400 instruments and are calibrated using residual undeuterated solvent $\left(\mathrm{CHCl}_{3}\right.$ at $7.26 \mathrm{ppm}{ }^{1} \mathrm{H} \mathrm{NMR}$, $77.16 \mathrm{ppm}{ }^{13} \mathrm{C}$ NMR), $\left(\mathrm{C}_{6} \mathrm{H}_{6}\right.$ at $7.16 \mathrm{ppm}{ }^{1} \mathrm{H}$ NMR, $\left.128.06{ }^{13} \mathrm{C} \mathrm{NMR}\right),\left(\mathrm{MeOH}\right.$ at $3.31 \mathrm{ppm}{ }^{1} \mathrm{H}$ NMR, $49.00 \mathrm{ppm}{ }^{13} \mathrm{C}$ NMR), (DMSO at $2.50 \mathrm{ppm}{ }^{1} \mathrm{H}$ NMR, $39.52 \mathrm{ppm}{ }^{13} \mathrm{C}$ NMR). The following abbreviations were used to explain multiplicities: $\mathrm{s}=$ singlet, $\mathrm{d}=$ doublet, $\mathrm{t}=$ triplet, $\mathrm{q}=$ quartet, $\mathrm{m}=$ multiplet, $\mathrm{br}=$ broad. Column chromatography was performed using E. Merck silica (60, particle size $0.043-0.063 \mathrm{~mm}$ ), and pTLC was performed on Merck silica plates (60F254). Highresolution mass spectra (HRMS) were recorded on an Agilent LC/MSD TOF mass spectrometer by electrospray ionization time of flight reflectron experiments. Melting points were recorded on a Fisher-Johns 12-144 melting point apparatus and are uncorrected.

\section{EARLY SYNTHETIC STRATEGIES}

\section{Decarboxylative Cross Coupling Disconnection}

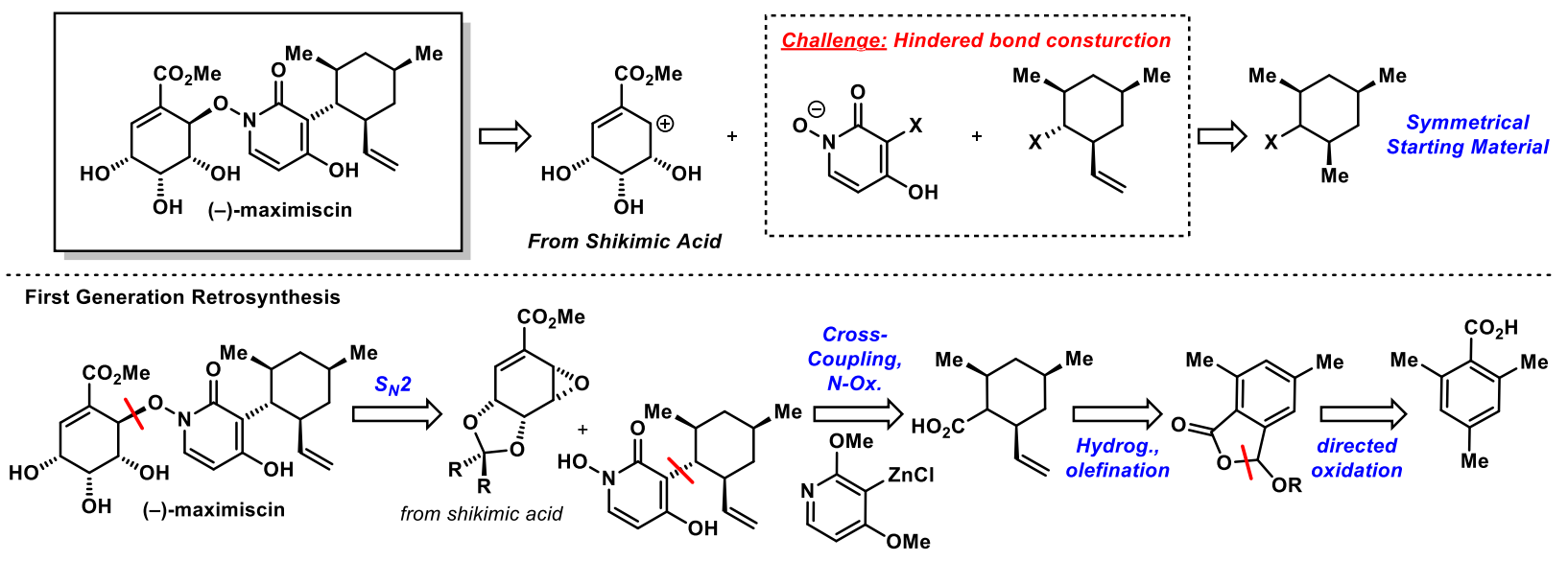




\section{Route to Carboxylic Acid Coupling Partner}

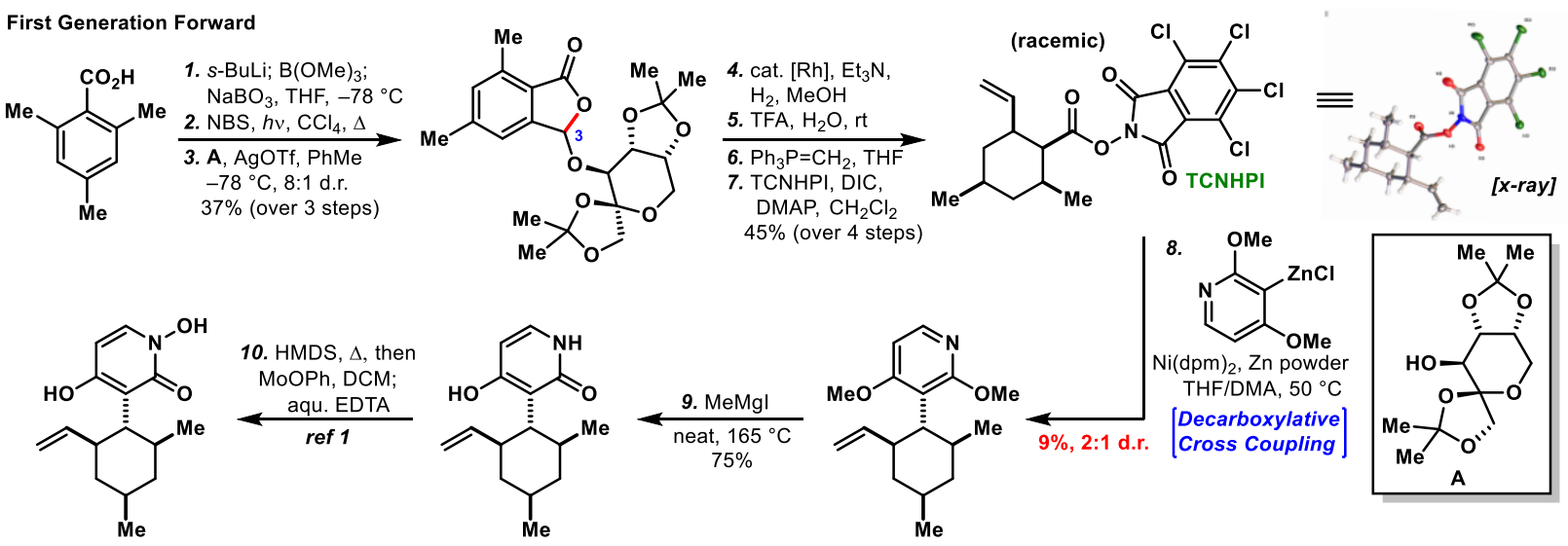

$( \pm)$-pyridoxatin

\section{Route Notes:}

- Step 1: Developed a safe, scalable directed benzylic C-H oxidation (see Racemic Synthesis 12 for detailed experimental)

- Step 3: Alcohol addition proceeded with moderate diastereoselectivity, relative configuration of major 3-hyroxyphthalide not determined.

- Step 4: Diastereoselective hydrogenation provided access to enantiopure material after auxiliary removal (step 5). Also solved hydrogenolysis problem occurring between C-3 and $\mathrm{O}$ (bond highlighted in red) which was prevalent when $\mathrm{C}-3$ substituent was methoxy. Triethylamine additive ( $25 \mathrm{~mol} \%$ ) was also found to help supress hydrogenolysis (using 5 $\mathrm{mol} \% \mathrm{Rh} / \mathrm{C}$ ). Note: Racemic material derived from 3-methoxy-phthalide carried forward for cross-coupling optimization.

- Step 8: $\mathrm{Ni}(\mathrm{dpm})_{2}$ uniquely successful at promoting the reaction. Zinc powder was required to access low valent nickel as the organozinc was too hindered to undergo homocoupling. Product obtained as mixture of atropisomers, mass balance was primarily decarboxylation.

\section{Decarboxylative Cross Coupling Optimization}

Selected Step 8 Optimization

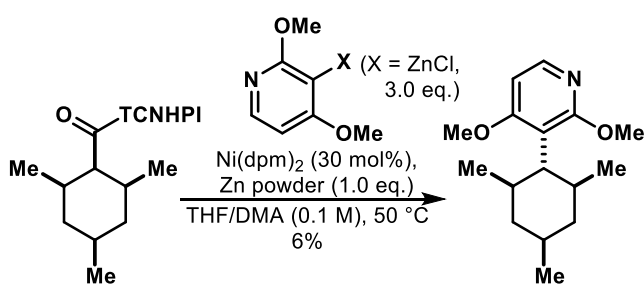

[model substrate]

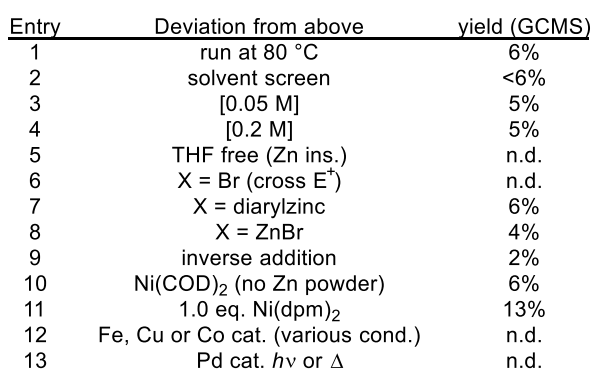

Precatalyst optimization - $\beta$-diketonate ligands $\quad \mathrm{R}=\mathrm{Ph}, \mathrm{CF}_{3}, \mathrm{Ad}, \mathrm{Me}$,

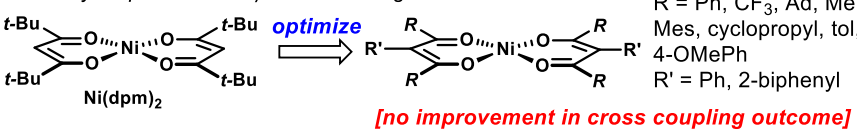

Precatalyst optimization - NHCs (cross coupling yields in brackets)

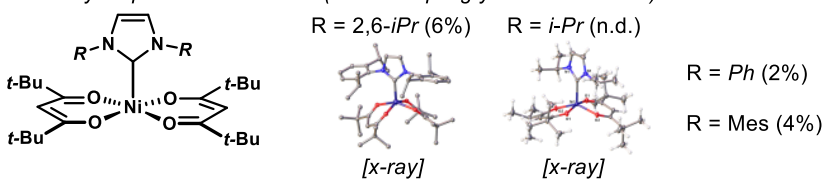

Selected ligand effects [30 mol\%] (>50 screened)
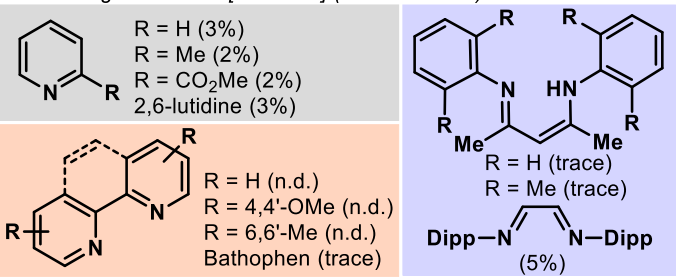

$\mathrm{PPh}_{3}$ (trace) $\mathrm{PCy}_{3}$ (n.d.) QPhos (7\%) DavePhos $(7 \%)$ CyJohnPhos (7\%) MePhos (9\%) BI-DIME $(4 \%)$ MonoPhos (trace)

Xantphos (n.d.) dppf (n.d.) 


\section{Optimization notes:}

- Extensive optimization studies conducted using model substrate.

- Generally, ligand free conditions worked best, although phosphines were tolerated and, in some cases offered marginal yield improvements. $\mathrm{Ni}(\mathrm{dpm})_{2}$ and $\mathrm{Ni}(\mathrm{COD})_{2}$ were the most effective catalysts surveyed, zinc powder was not required for the latter.

- The major by-product observed was consistently decarboxylation, reactions went to completion. Based on studies from Molander and co-workers it seems plausible the intermediate radical formed after decarboxylation is unable to intercept the hindered metal center and undergoes competing hydrogen atom transfer (HAT). ${ }^{2}$ After significant optimization efforts, this route was abandoned.

\section{Radical Pyridone Substitution Strategy}

Radical Addition: Minisci-Tactic
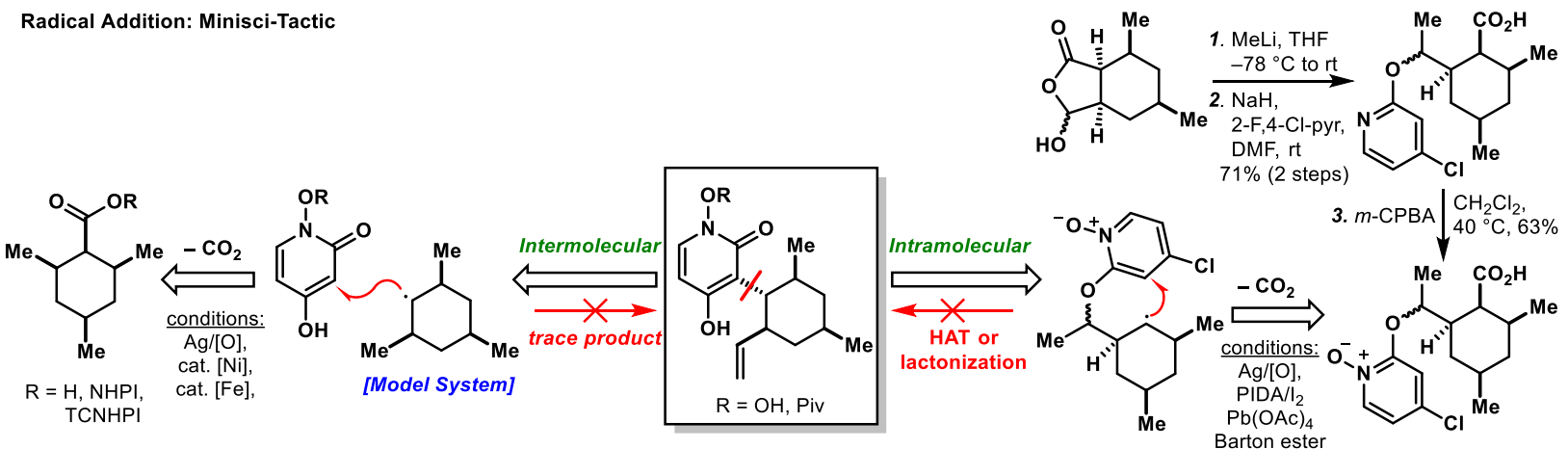

\section{Route notes:}

- Encouraged by the work or Miura involving selective C-3 radical addition into 2pyridones, ${ }^{3}$ we evaluated various intermolecular Minisci-type decarboxylative radical addition strategies. Unfortunately, we never observed significant product formation.

- An intramolecular strategy was evaluated, leveraging a previous synthetic intermediate which could be elaborated to a tethered pyridine- $N$-oxide in 3 steps. It was rationalized that under decarboxylative conditions, the intramolecularly tethered radicalphile would undergo substitution at C-3 to forge the hindered bond; subsequent elimination would unmask the $N$-hydroxypyridone motif. Unfortunately, no product was ever observed; generally the reactions gave non-productive decarboxylation or lactonization with expulsion of an $\mathrm{N}$-hydroxypyridone leaving group.

\section{Pyridone Scission Disconnection}

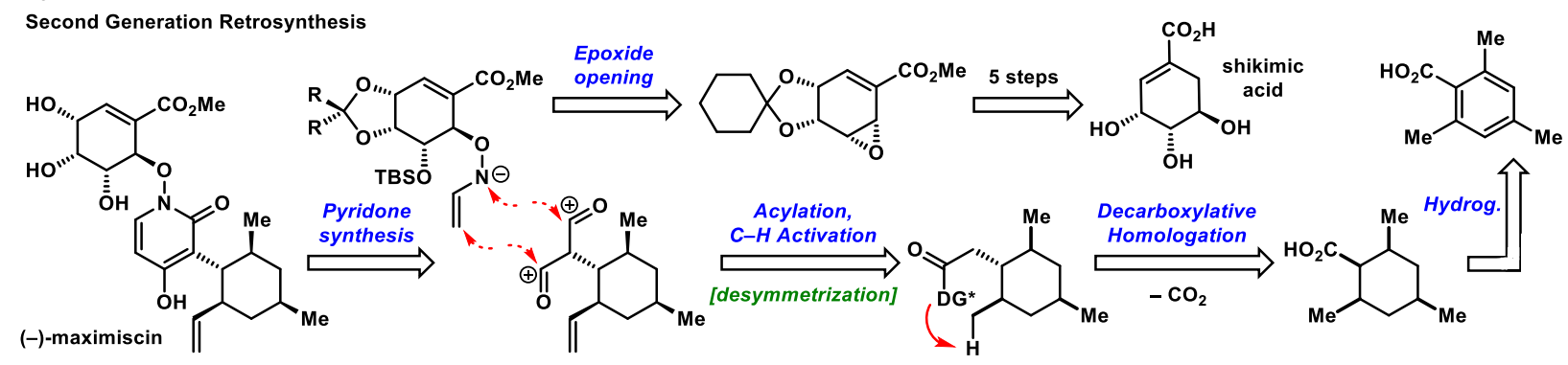




\section{C-H Activation via 7-Membered Metallacycle}

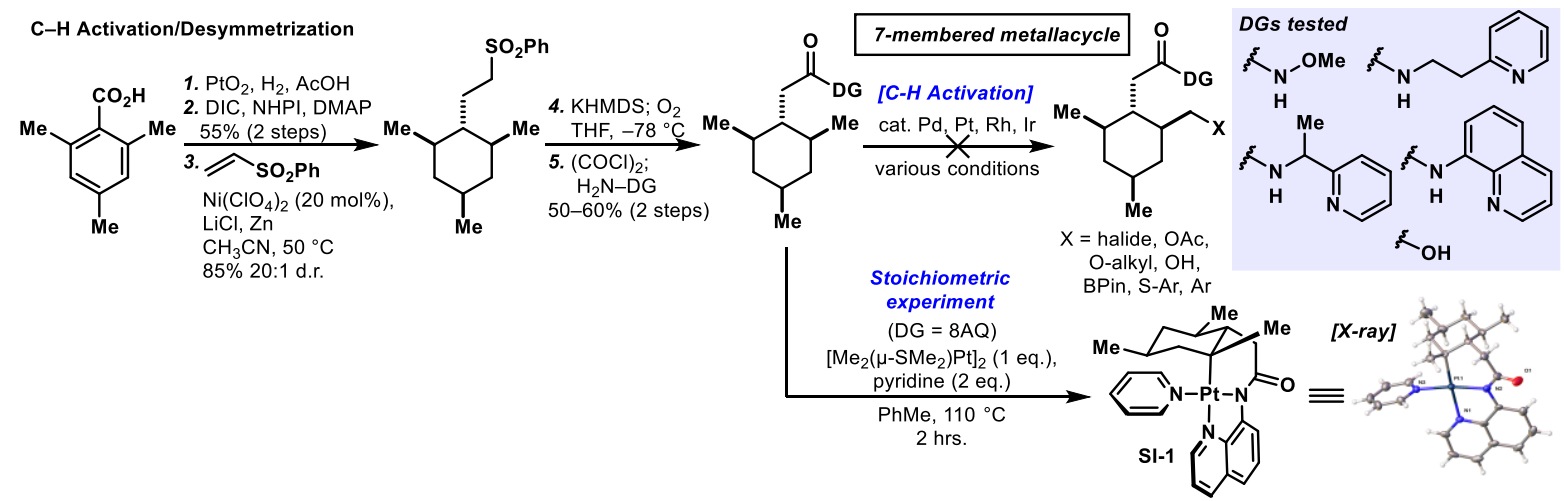

\section{Notes:}

- Despite trying numerous directing groups under various $\mathrm{C}-\mathrm{H}$ functionalization conditions, no methyl $\mathrm{C}-\mathrm{H}$ activation was observed

- Inspired by the work of $\operatorname{Sames}^{4}$ a stoichiometric experiment was conducted using $\left[\mathrm{Me}_{2}(\mu-\right.$ $\left.\left.\mathrm{SMe}_{2}\right) \mathrm{Pt}\right]_{2}$ resulting in an unusual tertiary 6-membered platinacycle SI-1. This was the only cyclometallated species identified from the reaction mixture. At this juncture, $\mathrm{C}-\mathrm{H}$ activation via a more precedented 6-membered metallacycle was targeted.

\section{C-H Activation via 6-Membered Metallacycle}
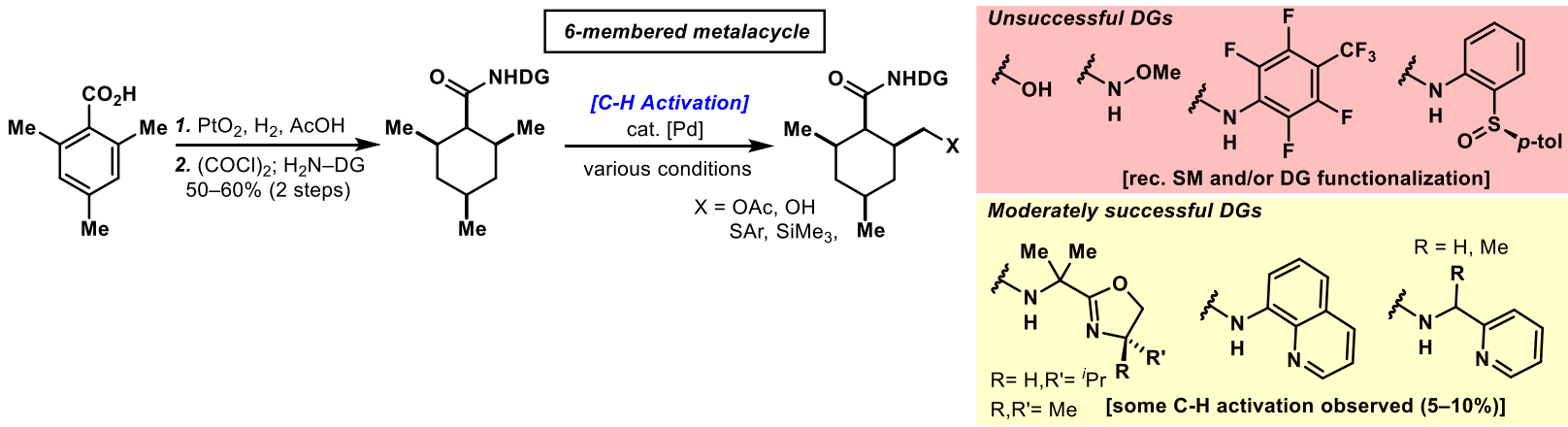

\section{Notes:}

- Numerous directing groups were evaluated under a variety of published $\mathrm{Pd}$ catalyzed $\mathrm{C}-\mathrm{H}$ functionalization conditions. Bidentate directing groups gave superior results, 8-AQ and PIP-type directing groups were most promising.

- Ultimately a tert-butyl substituted PIP-type directing group proved optimal, for detailed optimization studies see: REACTION OPTIMIZATION>C-H Activation (step 3). 


\section{Decarboxylative Homologation}

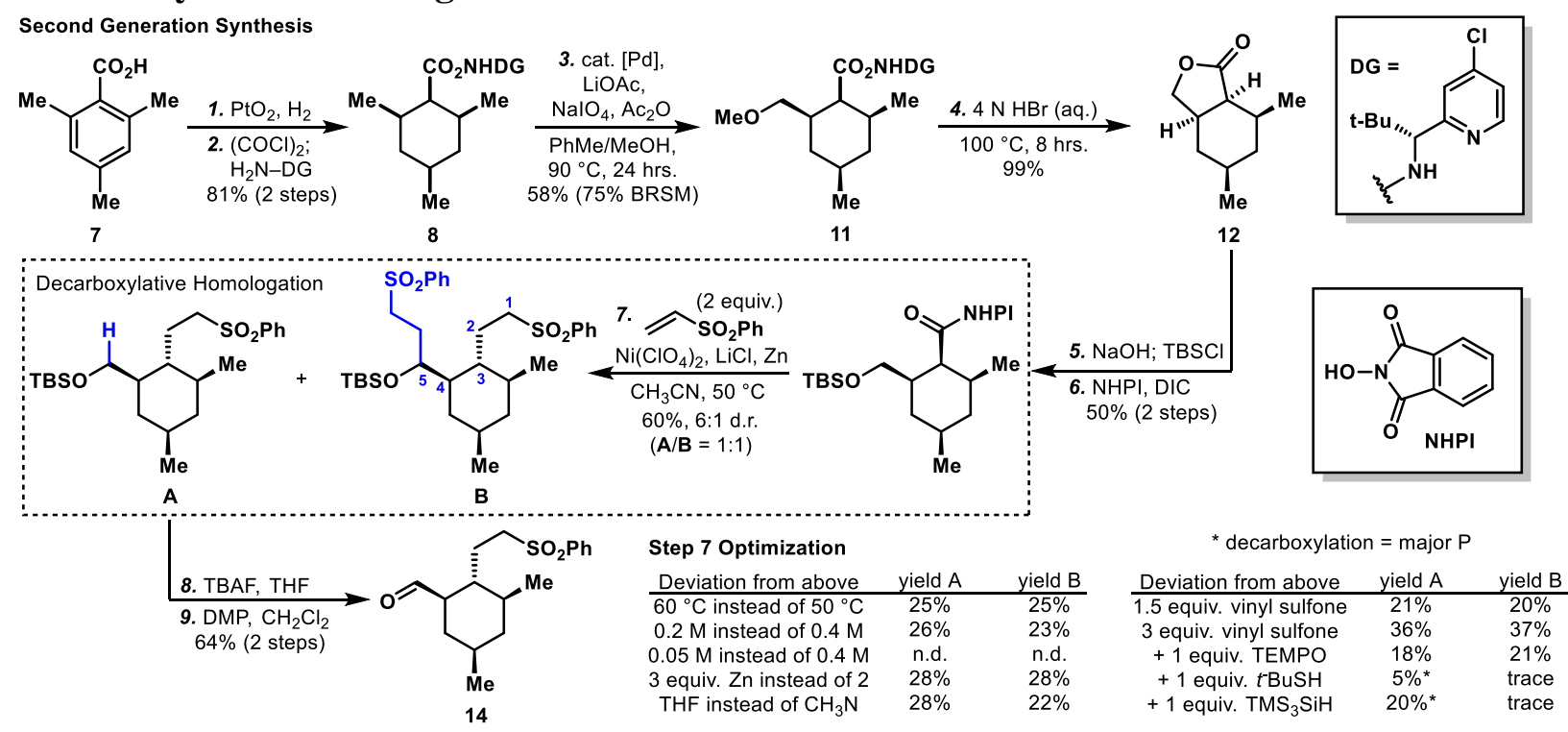

Support for 1,5-HAT Process

Confirm connectivity of double addition by-product
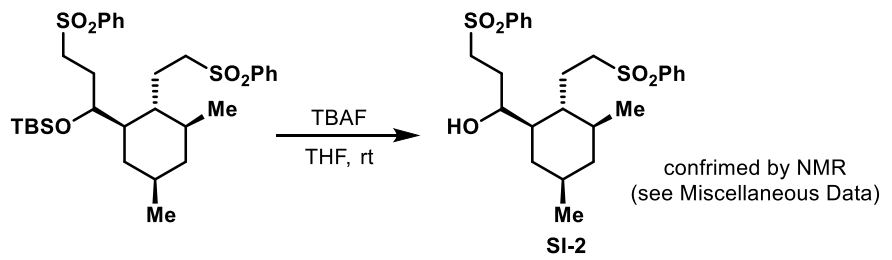

\section{Notes:}

- Step 7: Decarboxylative Giese addition led to a 1,5-HAT process generating an alpha oxy radical which underwent double addition with phenyl vinyl sulfone.

- Double addition 1,5-HAT product confirmed by NMR (see Miscellaneous data, NMR SPECTRA)

- No synthetically useful solutions found to supress 1,5-HAT under reductive conditions

- Opted to explore redox manifolds which could enable oxidation of the $\alpha$-oxy-radical intermediate to access aldehyde $\mathbf{1 4}$ directly. 


\section{Exotic Redox Strategies}

PET to Oxidatively Trap Radical Intermediate

Reactions conducted on $0.1 \mathrm{mmol}$ scale in degassed solvents, photoreactor prepared using blue LED strip (IEKOV TM 5050 SMD) and tin can, cooled under stream of air yields were obtained by NMR using dibromomethane as internal standard

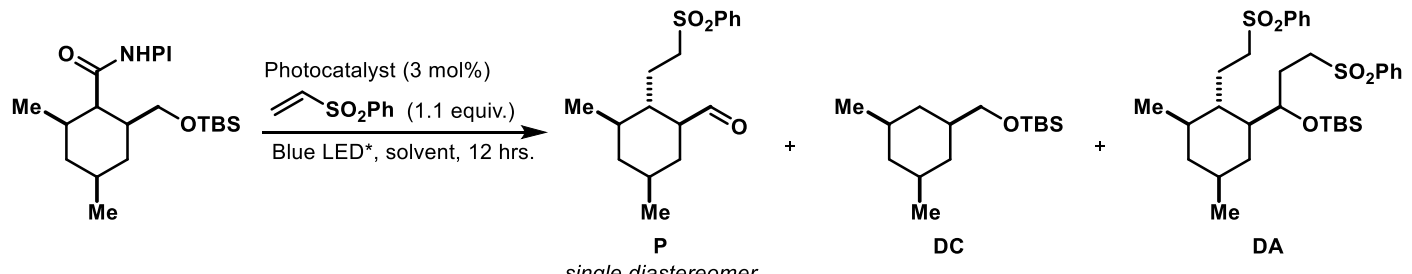

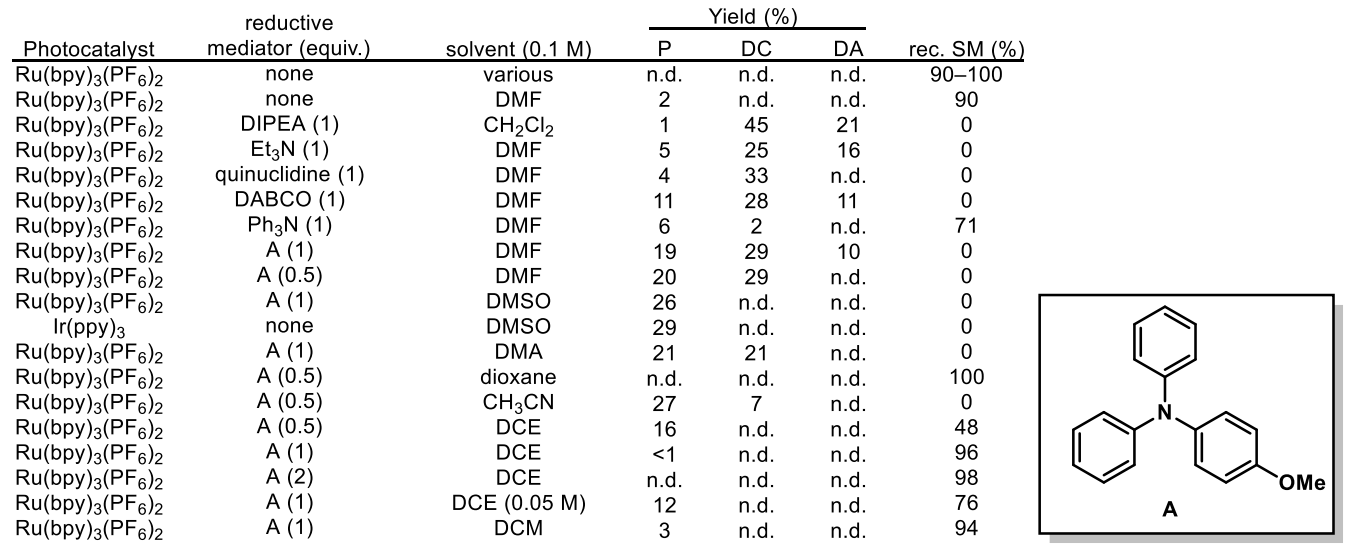

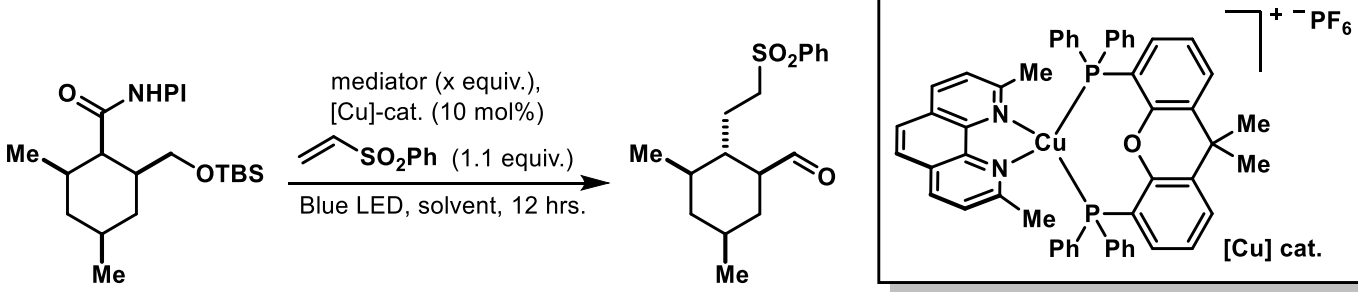

\begin{tabular}{cccc} 
mediator (equiv.) & solvent (M) & Yield (\%) (NMR) & rec. SM (\%) \\
\hline none & DMF (0.1) & n.r. & 98 \\
DABCO (1) & DMF (0.1) & 3 & 0 \\
A (1) & DMSO (0.1) & n.r. & 98 \\
A (1) & CH $_{3}$ CN (0.1) & n.r. & 97 \\
A (1) & DCM (0.1) & 9 & 49 \\
A (1) & DCM (0.1) & n.r. & 98
\end{tabular}

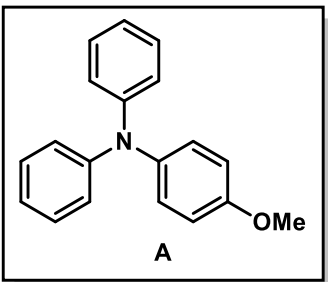




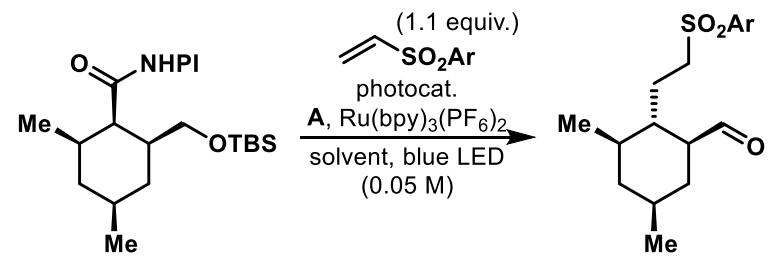

\begin{tabular}{|c|c|c|}
\hline $\mathrm{Ar}=$ & solvent & $\%$ yield \\
\hline $\mathrm{Ph}$ & $\mathrm{CH}_{3} \mathrm{CN}$ & 28 \\
\hline $\mathrm{Ph}$ & $\mathrm{CH}_{3} \mathrm{CN}(0.025 \mathrm{M})$ & 23 \\
\hline $\mathrm{Ph}$ (1.5 equiv.) & $\mathrm{CH}_{3} \mathrm{CN}$ & 28 \\
\hline $\mathrm{Ph}$ & DMSO & 26 \\
\hline BT & $\mathrm{CH}_{3} \mathrm{CN}$ & 19 \\
\hline BT & DMSO & 18 \\
\hline PT & $\mathrm{CH}_{3} \mathrm{CN}$ & 24 \\
\hline PT & DMSO & 13 \\
\hline
\end{tabular}

\section{Additive}

\begin{tabular}{lc} 
Additive (in $\left.\mathrm{CH}_{3} \mathrm{CN}\right)$ & $\%$ yield \\
\hline $\mathrm{LiBF}_{4}(1$ equiv. & 4 \\
$\mathrm{La}(\mathrm{OTf})_{3}(1$ equiv. & messy \\
$\mathrm{Cu}(\mathrm{OTFA})_{2}$ ( 0.1 equiv.) & trace \\
pyrene $(0.15$ equiv.) & 23 \\
$\mathrm{H}_{2} \mathrm{O}$ (10 equiv.) & 30
\end{tabular}

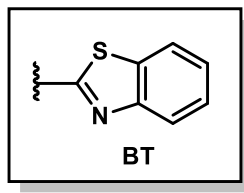

Solvent

Additive/Modification

\begin{tabular}{lc} 
solvent & \% vield \\
\hline $\mathrm{MeOAc} / \mathrm{CH}_{3} \mathrm{CN}(3.5: 1)$ & 16 \\
$\mathrm{MeOAc} / \mathrm{CH}_{3} \mathrm{CN}(4: 1)$ & $20(12 \mathrm{hrs})$. \\
$\mathrm{MeOAc} / \mathrm{CH}_{3} \mathrm{CN}(4: 1)$ & $28(24 \mathrm{hrs})$. \\
$\mathrm{MeOAc/CH} 3 \mathrm{CN}(1: 1)$ & 30 \\
$\mathrm{MeOAc} / \mathrm{DMSO}(4: 1)$ & 36 \\
$\mathrm{MeOAc/DMSO}(6: 1)$ & 34 \\
$\mathrm{MeOAc} / \mathrm{DMSO}(9: 1)$ & 35 \\
DMC/DMSO (9:1) & 24 \\
TFT/DMSO (6:1) & 33
\end{tabular}

\section{MeOAc/DMSO (6:1)}

+10 equiv. $\mathrm{H}_{2} \mathrm{O}$

+10 equiv. $\mathrm{H}_{2} \mathrm{O}$

+20 equiv. $\mathrm{H}_{2} \mathrm{O}$

+10 equiv. HFIP

0.5 eq. $\mathbf{A}+10$ equiv. HFIP

0.5 eq. $\mathbf{A}+10$ equiv. $\mathrm{H}_{2} \mathrm{O}$

sulfolane instead of DMSO

- reaction messy (suspect aldehyde decomp.)

- potential scalability issues

Organic Dye

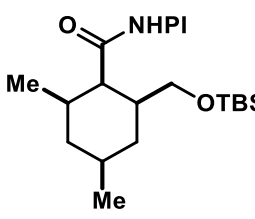

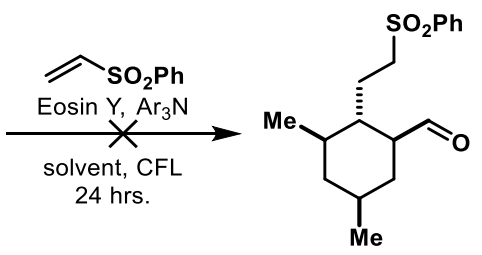<smiles></smiles><smiles>CC1CC(C)[C@H](CCS(=O)(=O)c2ccccc2)C(C=O)C1</smiles>

Kolbe Electrolysis Directly From Free Carboxylic Acid

Kolbe reactions were conducted using an ElectraSyn 2.0 in $1 \mathrm{ml}$ Micro Glass vials using platinum foil electrodes

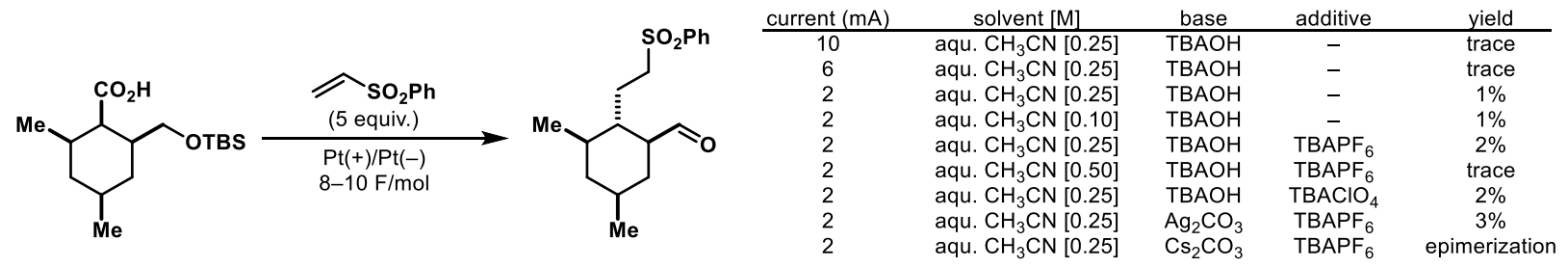

mass balance:

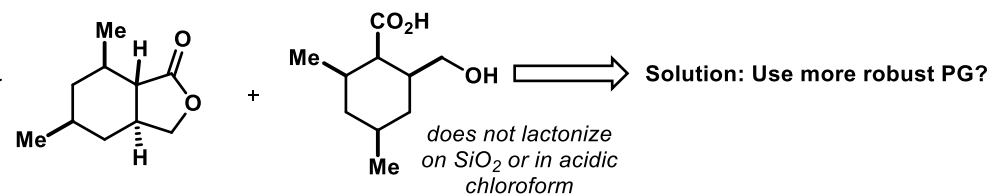




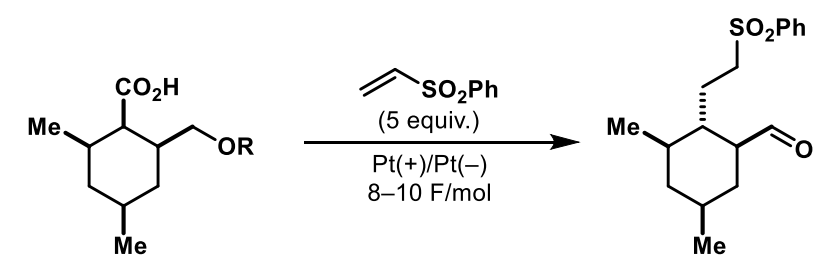

\begin{tabular}{|c|c|c|c|c|c|}
\hline $\mathrm{R}$ & current ( $m A$ ) & solvent [M] & base & additive & yield \\
\hline $\mathrm{Me}$ & 2 & aqu. $\mathrm{CH}_{3} \mathrm{CN}[0.25]$ & TBAOH & TBAPF $_{6}$ & rec SM \\
\hline TBDPS & 2 & aqu. $\mathrm{CH}_{3} \mathrm{CN}[0.25]$ & $\mathrm{TBAOH}$ & TBAPF $_{6}$ & rec. SM \\
\hline TBS & 10 & $\mathrm{DCM} / \mathrm{CH}_{3} \mathrm{CN} / \mathrm{HFIP}[0.2]$ & 2,4,6-colidine & $\mathrm{TBAPF}_{6}, \mathrm{AgPF}_{6}$ & rec SM \\
\hline TBS & 4 & $\mathrm{CH}_{3} \mathrm{CN} / \mathrm{HFIP}[0.25]$ & $\mathrm{Ag}_{2} \mathrm{CO}_{3}$ & TBAPF $_{6}$ & n.d. \\
\hline $\mathrm{Me}$ & 10 & $\mathrm{MeOH}[0.25]$ & $\mathrm{KOH}$ & - & rec SM \\
\hline $\mathrm{Me}$ & 50 & $\mathrm{MeOH}[0.25]$ & $\mathrm{KOH}$ & - & rec. SM, sulfone consumed \\
\hline $\mathrm{Me}$ & 50 & aqu. $\mathrm{CH}_{3} \mathrm{CN}[0.25]$ & $\mathrm{TBAOH}$ & - & rec. SM, sulfone consumed \\
\hline TBDPS & 50 & $\mathrm{MeOH}[0.25]$ & $\mathrm{KOH}$ & - & rec. SM, sulfone consumed \\
\hline
\end{tabular}




\section{REACTION OPTIMIZATION}

\section{C-H Activation (Step 3)}

Reactions were run in small culture tubes sealed with a teflon screw cap, Pd loading $=20 \mathrm{~mol} \%$ using $\mathrm{Pd}(\mathrm{OAc})_{2}, 3$ equiv. PIDA, $0.1 \mathrm{M}, 24 \mathrm{hrs}$, unless otherwise stated. Yields were quantified by GCMS relative to dodecane internal standard or by NMR using dibromomethane as internal standard.

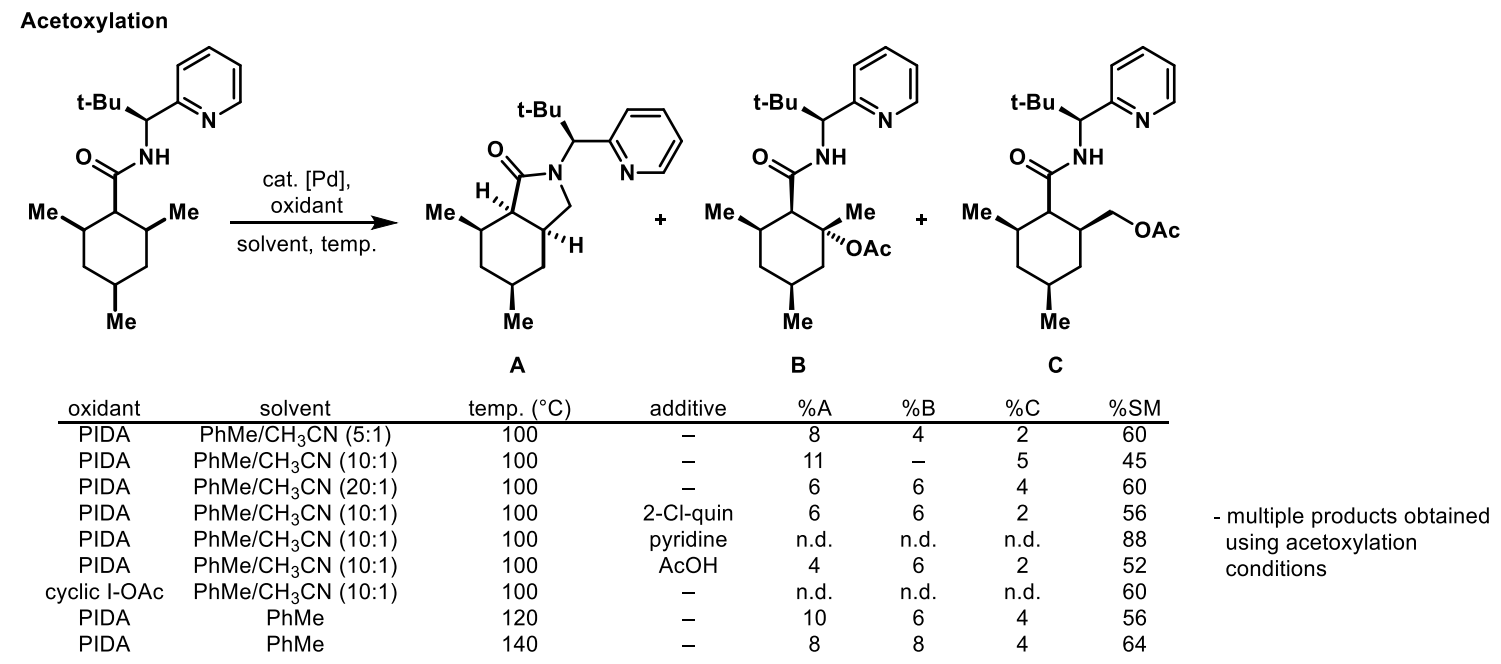

Alkoxylation

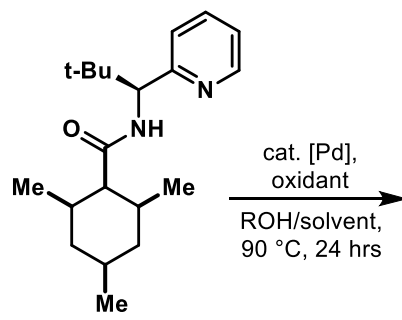

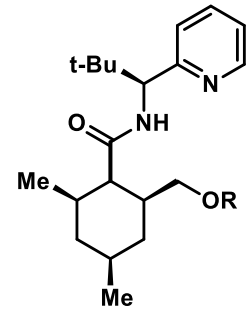

\begin{tabular}{cccc} 
oxidant & $\mathrm{ROH} /$ solvent & $\% \mathrm{P}$ & $\% \mathrm{SM}$ \\
\hline PIDA & BnOH/PhMe $(1: 1)$ & n.d. & - \\
PIDA & MeOH/PhMe (1:1) & 23 & - \\
PIDA & MeOH/PhMe (2:1) & 13 & 86 \\
PIDA & MeOH/PhMe (1:2) & 25 & 69 \\
PIDA & MeOH/PhMe (1:4) & 15 & 60 \\
PIDA & MeOH/PhMe (1:8) & 5 & 57
\end{tabular}

- methoxylation gives single

major product

- high d.r. (>10:1) 


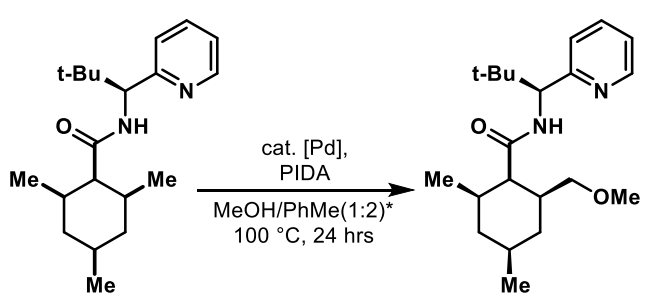

Ligand Screen

$33 \%$ (34\% SM)<smiles>Clc1ccccn1</smiles>

$40 \%(41 \%$ SM)<smiles>Oc1ccc2ccccc2n1</smiles>

$24 \%(46 \%$ SM)

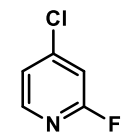

$35 \%(34 \% \mathrm{SM})$

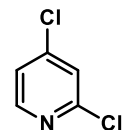

$37 \%(37 \%$ SM)

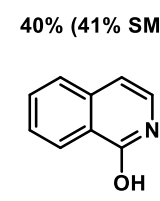

$32 \%$ $28 \%(66 \% \mathrm{SM})$
Solvent screen (MeOH/solv. 1:1.5)*

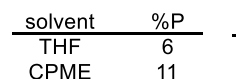

CPME 11

MTBE

dioxane

DME

$\begin{array}{ll}\mathrm{CH}_{3} \mathrm{CN} & 2\end{array}$

NMP trace

DMF

t-BuOH 6

HFIP trace

benzene 14

PhMe 18

TFT

$\mathrm{PhCl}$

DCE trace

$\mathrm{MeOAc} \quad 9$

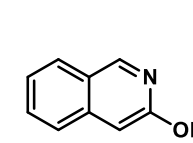

$23 \%(67 \%$ SM)<smiles>Cc1c2c(nc3ccc(C(C)(C)C)cc13)OC(C)CC2</smiles><smiles>Nc1cccc(N)n1</smiles>

$5 \%$<smiles>c1cnc2c(c1)ccc1cccnc12</smiles>

Base screen (1 equiv.)

\begin{tabular}{ccc} 
Base & $\% \mathrm{P}$ & $\% \mathrm{SM}$ \\
\hline $\mathrm{NaOMe}$ & 27 & 59 \\
$\mathrm{Na}_{2} \mathrm{CO}_{3}$ & 14 & 69 \\
$\mathrm{NaHCO}_{3}$ & 33 & 52
\end{tabular}

$\mathrm{NaOPh}$

$\mathrm{K}_{3} \mathrm{PO}_{4}$

$\mathrm{K}_{2} \mathrm{HPO}_{4}$

$\mathrm{KH}_{2} \mathrm{PO}_{4}$

$\mathrm{KHCO}_{3}$

KOAC

LIOAC 43

LiOAc $(2.0$

$\mathrm{Li}_{2} \mathrm{CO}_{3}$
$\mathrm{LiOt}-\mathrm{Bu}$

$\mathrm{LiOH} \times \mathrm{H}_{2} \mathrm{O}$

$\mathrm{Ag}_{2} \mathrm{CO}_{3}$

$\mathrm{AgOAC}$

$\mathrm{Cs}_{2} \mathrm{CO}_{3}$

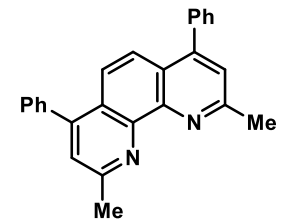

$25 \%(67 \% \mathrm{SM})$<smiles>c1ccc(-c2ccccn2)nc1</smiles>

Directing Group Evaluation

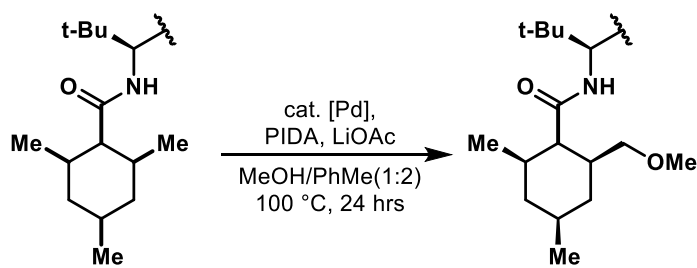<smiles>Cc1cc(Cl)ccn1</smiles>

$53 \%$<smiles>Cc1ccnc(I)c1</smiles><smiles>CCc1cccc(OC)n1</smiles>

mal co-solvent - Li bases perform best

$-\mathrm{Pd}(\mathrm{OAc})_{2}$ adequate cat.

- no ligand acceleration

Screening Reaction Parameters and Additives<smiles>[M]C1CC(C)C(C(=O)NC(c2cc(Cl)ccn2)C2CCCCC2C(=O)OC)C(COC)C1</smiles>

\begin{tabular}{|c|c|c|c|c|c|}
\hline \multirow[b]{2}{*}{ Alt. Base } & \multirow[b]{2}{*}{$\% \mathrm{P}$} & \multirow[b]{2}{*}{ Pd loading } & \multirow[b]{2}{*}{$\% \mathrm{P}$} & \multicolumn{2}{|c|}{ Pd loading $=10 \mathrm{~mol} \%$} \\
\hline & & & & conc. (M) & $\% \mathrm{P}$ \\
\hline LiOMe & 52 & $20 \mathrm{~mol} \%$ & 53 & 0.8 & 5 \\
\hline LiOPiv & 50 & $10 \mathrm{~mol} \%$ & 27 & 0.6 & 6 \\
\hline LiOTFA & 31 & $5 \mathrm{~mol} \%$ & 16 & 0.4 & 12 \\
\hline LiHMDS & 11 & & & 0.2 & 18 \\
\hline $\mathrm{Li}_{3} \mathrm{PO}_{4}$ & 33 & & & 0.1 & 28 \\
\hline $\mathrm{LiH}_{2} \mathrm{PO}_{4}$ & 14 & & & 0.05 & 34 \\
\hline $\mathrm{Li}_{2} \mathrm{SO}_{4}$ & 15 & & & & \\
\hline LiOBz & 49 & & & $\mathrm{Ac}_{2} \mathrm{O}$ (equiv.) & $\% \mathrm{P}$ \\
\hline & \multirow{4}{*}{\multicolumn{3}{|c|}{$\begin{array}{l}\text { - lower concentration improves yield } \\
\text { - } \mathrm{Ac}_{2} \mathrm{O} \text { improves yield, } 2 \text { equiv. chosen, } \\
\text { higher loadings reduced } \mathrm{BRSM} \text { yield }\end{array}$}} & 1 & 37 \\
\hline & & & & 2 & 39 \\
\hline & & & & 3 & 40 \\
\hline & & & & 5 & 39 \\
\hline
\end{tabular}

The role of $\mathrm{Ac}_{2} \mathrm{O}$ is unclear. It is a common additive used in numerous Pd catalyzed $\mathrm{C}-\mathrm{H}$ acetoxylation/alkoxylation reactions. ${ }^{5}$ We did find that the $\mathrm{C}-\mathrm{H}$ activation reaction was somewhat sensitive to moisture, so it may be serving as a desiccant. 


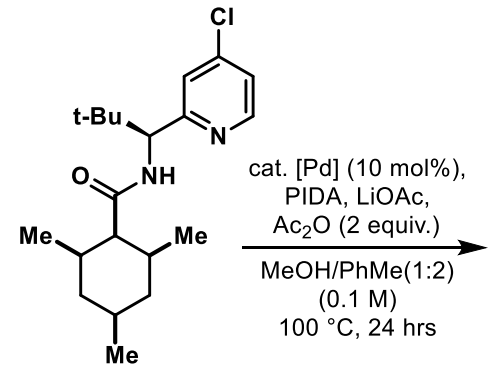<smiles>[M]C1CC(C)C(C(=O)NC(c2cc(Cl)ccn2)C(C)(C)C)C(COC)C1</smiles>

change from left $\mathrm{Pd}(\mathrm{OPiv})_{2}$ instead of $\mathrm{Pd}(\mathrm{OAc})_{2} \quad 37$ $\mathrm{Pd}_{2}(\mathrm{dba})_{3}$ instead of $\mathrm{Pd}(\mathrm{OAc})_{2} \quad 37$ $2 \times 5 \mathrm{~mol} \%[\mathrm{Pd}](t=0,12 \mathrm{hrs})$ $\mathrm{Piv}_{2} \mathrm{O}$ instead of $\mathrm{Ac}_{2} \mathrm{O}$ $\mathrm{AcOH}$ (1 equiv.) DMC (1 equiv.)

- $\mathrm{NaIO}_{4}$ preferable to PIDA (cost, ease of purification)

\begin{tabular}{ccccc}
$\begin{array}{c}\mathrm{NalO}_{4} \\
\text { (equiv.) }\end{array}$ & $\begin{array}{c}\mathrm{Pd} \\
\text { (mol\%) }\end{array}$ & $\begin{array}{c}\text { time } \\
\text { (hrs) }\end{array}$ & \%P & TON \\
\hline 3 & 10 & 48 & 39 & - \\
4 & 10 & 24 & 43 & - \\
5 & 10 & 24 & 37 & - \\
3 & 10 & 24 & 39 & 3.9 \\
3 & 15 & 24 & 51 & 3.4 \\
3 & 20 & 24 & 57 & 2.9
\end{tabular}

\begin{tabular}{|c|c|}
\hline \multirow{2}{*}{\multicolumn{2}{|c|}{$\frac{\text { oxidant (3 eq.) }}{\text { PIFA }}$}} \\
\hline & \\
\hline PhlO & trace \\
\hline TBHP & n.r. \\
\hline oxone & n.r. \\
\hline DMP & n.r. \\
\hline $\mathrm{K}_{2} \mathrm{~S}_{2} \mathrm{O}_{8}$ & n.r. \\
\hline NFSI & n.r. \\
\hline $\mathrm{NalO}_{4}$ & 39 \\
\hline $\mathrm{NaBO}_{3} \times \mathrm{H}_{2} \mathrm{O}$ & n.r. \\
\hline $\mathrm{I}_{2} \mathrm{O}_{5}$ & n.r. \\
\hline IBX & n.r. \\
\hline $\mathrm{LilO}_{4} \times 2 \mathrm{H}_{2} \mathrm{O}$ & n.r. \\
\hline $\mathrm{NBu}_{4} \mathrm{IO}_{4}$ & n.r. \\
\hline$t$-BuOOAC & n.r. \\
\hline
\end{tabular}

using 4 equiv. $\mathrm{NaIO}_{4}$

\begin{tabular}{cc} 
Additive $(0.1$ equiv. $) \quad \% \mathrm{P}$ \\
\hline $\mathrm{Cu}(\mathrm{OAC})_{2}$
\end{tabular} $\begin{array}{ll}\mathrm{Cu}(\mathrm{OAc})_{2} & 31 \\ \mathrm{Fe}(\mathrm{OAc})_{2} & 24\end{array}$ $\mathrm{Mn}(\mathrm{OAC})_{2}$

\begin{tabular}{cc} 
Temp. $\left({ }^{\circ} \mathrm{C}\right)$ & $\% \mathrm{P}$ \\
\hline 80 & 40 \\
90 & 45 \\
110 & 43 \\
120 & 42
\end{tabular}

Final Conditions<smiles>[M]C1CC(C)C(C(=O)NC(c2cc(Cl)ccn2)C(C)(C)C)C(COC)C1</smiles>

\section{Decarboxylative Giese Addition (Step 5)}

Optimization studies were carried out using racemic material on $0.1 \mathrm{mmol}$ scale unless otherwise stated. Yields were determined by NMR using dibromomethane as internal standard.

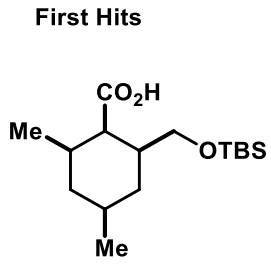

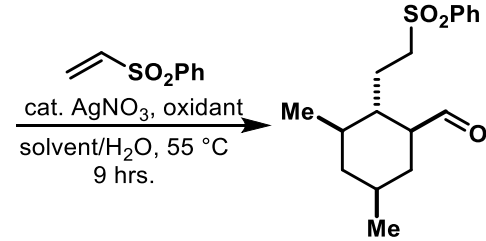

$\mathrm{K}_{2} \mathrm{~S}_{2} \mathrm{O}_{8}, \mathrm{CH}_{3} \mathrm{CN}=6 \%$

selectfluor, acetone $=10 \%+6 \%$ aldehyde epimer

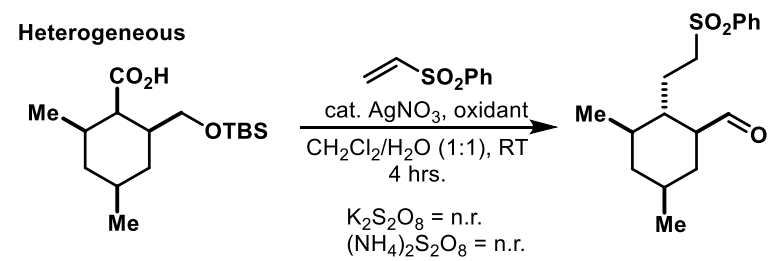

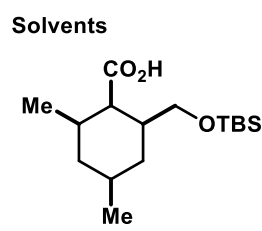

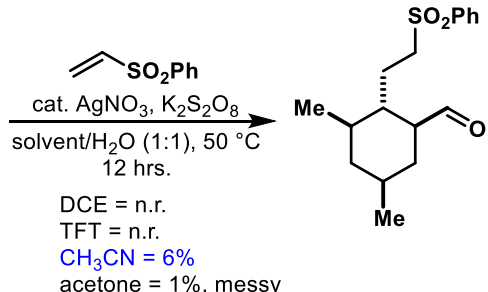



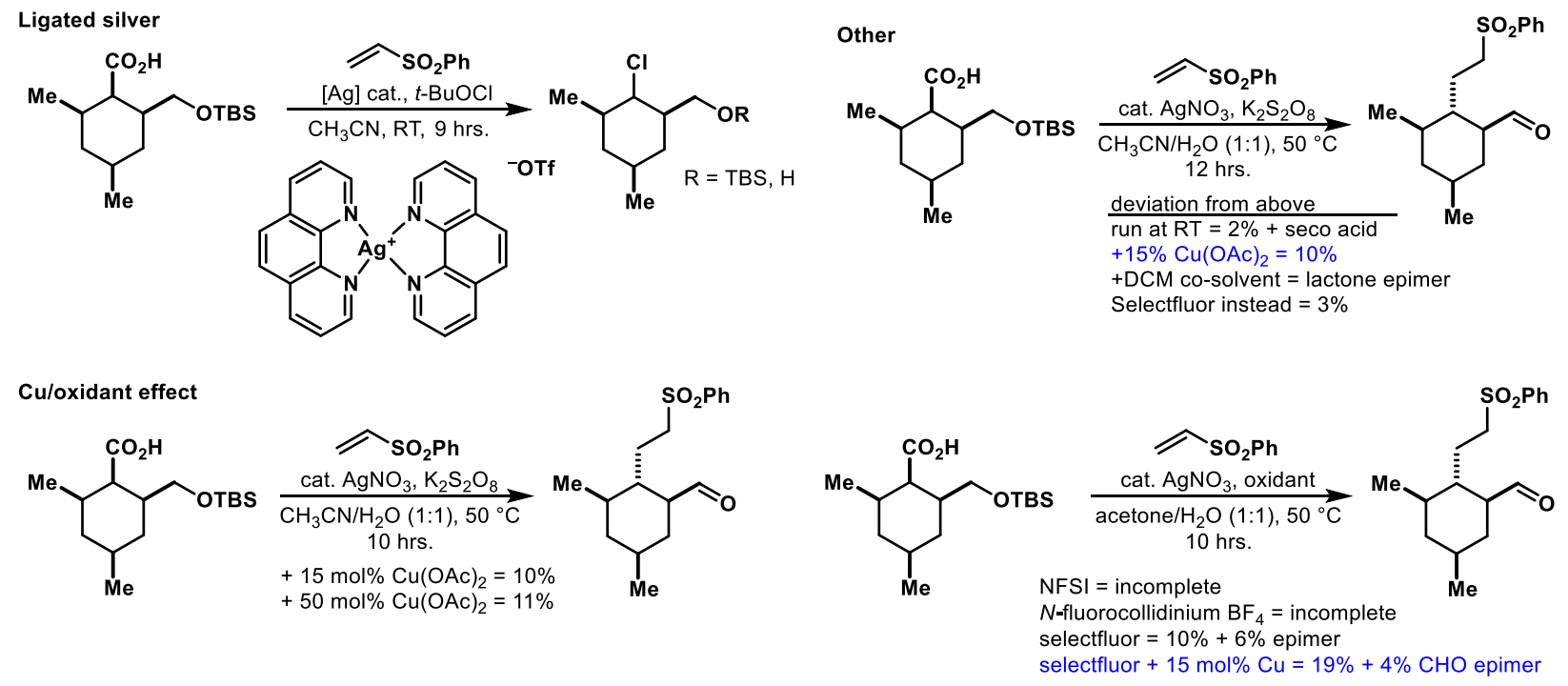

Estabilishing Reactive
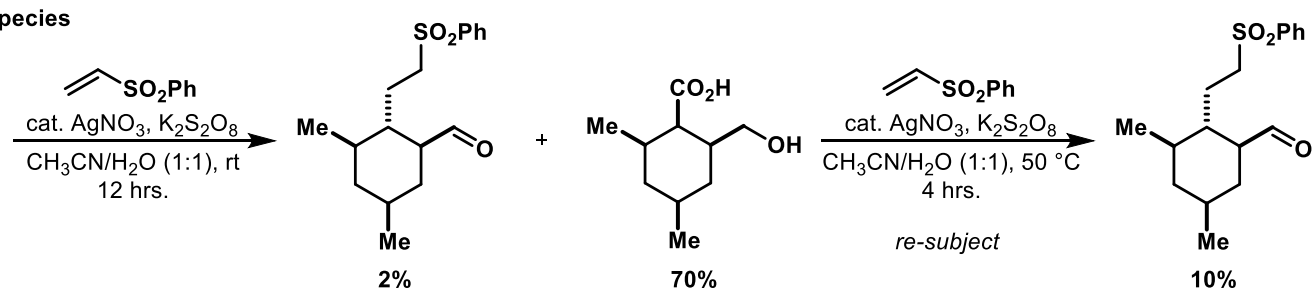

- seco-acid consumed - TBS PG not required
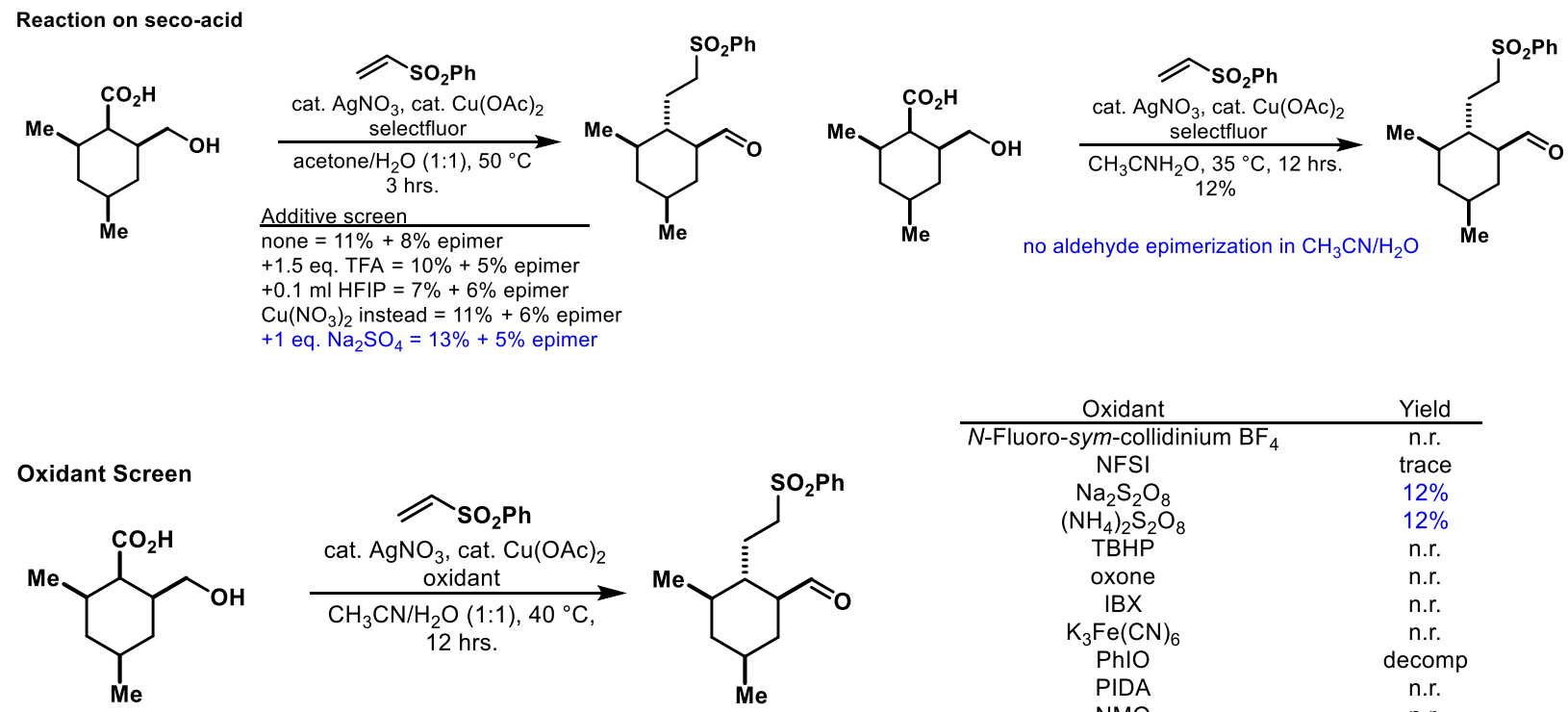

\begin{tabular}{cc} 
Oxidant & Yield \\
\hline$N$-Fluoro-sym-collidinium $\mathrm{BF}_{4}$ & n.r. \\
$\mathrm{NFSI}$ & trace \\
$\mathrm{Na}_{2} \mathrm{~S}_{2} \mathrm{O}_{8}$ & $12 \%$ \\
$\left(\mathrm{NH}_{4}\right)_{2} \mathrm{~S}_{2} \mathrm{O}_{8}$ & $12 \%$ \\
$\mathrm{TBHP}$ & n.r. \\
oxone & n.r. \\
IBX & n.r. \\
$\mathrm{K}_{3} \mathrm{Fe}(\mathrm{CN})_{6}$ & n.r. \\
$\mathrm{PhIO}$ & decomp \\
$\mathrm{PIDA}$ & n.r. \\
$\mathrm{NMO}$ & n.r. \\
$\mathrm{NaOCl}$ & n.r.
\end{tabular}


Ligated cat.<smiles>CC1CC(C)C(C(=O)O)C(CO)C1</smiles>

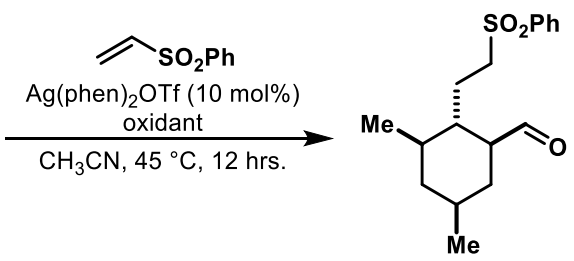

\begin{tabular}{cc} 
Oxidant & Yield \\
\hline TBHP & n.r. \\
PIDA & n.r. \\
$\mathrm{Na}_{2} \mathrm{~S}_{2} \mathrm{O}_{8}$ & epi-lactone
\end{tabular}

Stoichiometry<smiles>CC1CC(C)C(C(=O)O)C(CO)C1</smiles><smiles>CC1CC(C)[C@@H](CCS(=O)(=O)Oc2ccccc2)C(C=O)C1</smiles>

\begin{tabular}{cccc} 
Sulfone (equiv.) & Persulfate (equiv.) & Concentration [M] & Yield \\
\hline 1.2 & 2.5 & 0.1 & $14 \%$ \\
3 & 2.5 & 0.1 & $15 \%$ \\
5 & 5 & 0.1 & $15 \%$ \\
2 & 2 & 0.1 & $14 \%$ \\
2 & 3 & 0.1 & $15 \%$ \\
2 & 5 & 0.1 & $14 \%$ \\
2 & 2.5 & 0.05 & $15 \%$ \\
2 & 2.5 & 0.2 & $15 \%$ \\
2 & 2.5 & 0.1 & $15 \%{ }^{*}$ \\
& $*$ & &
\end{tabular}

Solvent Ratios<smiles>CC1CC(CO)C(C(=O)O)C([N+](=O)[O-])C1</smiles>

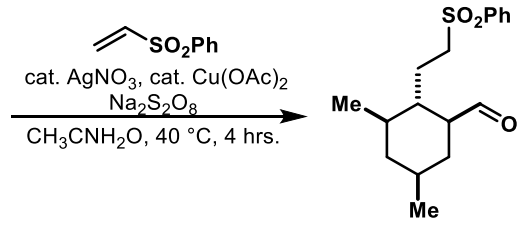

\begin{tabular}{cc}
$\mathrm{CH}_{3} \mathrm{CN} / \mathrm{H}_{2} \mathrm{O}$ ratio & yield \\
\hline $9: 1$ & $8 \%$ \\
$4: 1$ & $11 \%$ \\
$2: 1$ & $11 \%$ \\
$1: 2$ & $22 \%$ \\
$1: 4$ & $22 \%$ \\
$1: 9$ & $22 \%$
\end{tabular}

- higher water fraction improves reaction

Copper Source<smiles>CC1CC(C)C(C(=O)O)C(CO)C1</smiles>

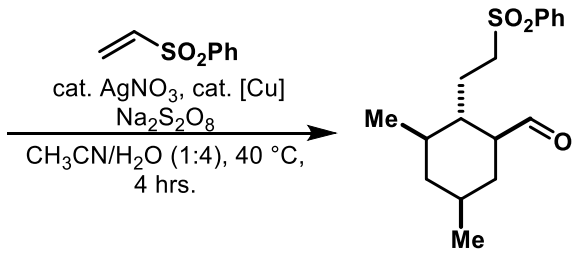

\begin{tabular}{cc} 
Copper & Yield \\
\hline $\mathrm{Cu}(\mathrm{acac})_{2}$ & $23 \%$ \\
$\mathrm{CuSO}{ }_{4} \times 5 \mathrm{H}_{2} \mathrm{O}$ & $20 \%$ \\
$\mathrm{Cu}(\mathrm{OTf})_{2}$ & $22 \%$ \\
$\mathrm{Cu}(\mathrm{OTFA})_{2}$ & $21 \%$ \\
$\mathrm{Cu}\left(\mathrm{CH}_{3} \mathrm{CN}\right)_{4} \mathrm{PF}_{6}$ & $23 \%$
\end{tabular}

Silver Source<smiles>CC1CC(CO)C(C(=O)O)C(CO)C1</smiles>

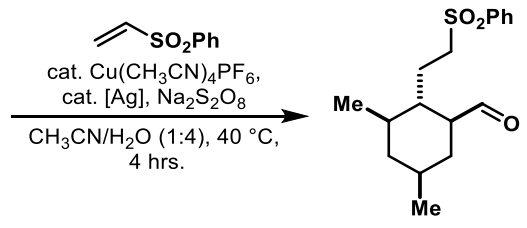

\begin{tabular}{cc} 
Silver & Yield \\
\hline none & n.d. \\
$\mathrm{AgOTf}$ & $22 \%$ \\
$\mathrm{Ag}_{2} \mathrm{CO}_{3}$ & $19 \%$ \\
$\mathrm{AgOAc}_{\mathrm{AgPF}}$ & $23 \%$ \\
$\mathrm{Ag}_{2} \mathrm{O}$ & $20 \%$ \\
$\mathrm{AgF}$ & $22 \%$ \\
$\mathrm{AgF}$ & $21 \%$
\end{tabular}

Co-catalyst Screen ${ }_{\mathrm{SO}_{2} \mathrm{Ph}}^{(2 \text { eq. }}$ $\mathrm{AgNO}_{3}(30 \mathrm{~mol} \%)$ [M] $(20 \mathrm{~mol} \%)$<smiles>C[C@@H]1CC(C(=O)O)C(CO)C([N+](=O)[O-])C1</smiles>
$\mathrm{Na}_{2} \mathrm{~S}_{2} \mathrm{O}_{8}$ (2.5 equiv.) $\mathrm{CH}_{3} \mathrm{CN} / \mathrm{H}_{2} \mathrm{O}(1: 4), 40^{\circ} \mathrm{C}$, $3 \mathrm{hrs}$.<smiles>C[C@@H]1CC[C@@H](CCS(=O)(=O)c2ccccc2)C(C=O)C1</smiles>

Iron Co-catalyst Screen<smiles>CC1C[C@@H](C)CC(CO)C1C(=O)O</smiles>

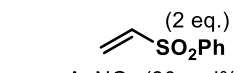
( 2 eq.) $\mathrm{AgNO}_{3}(30 \mathrm{~mol} \%)$, [Fe] $(20 \mathrm{~mol} \%)$, $\underset{\mathrm{CH}_{3} \mathrm{CN} / \mathrm{H}_{2} \mathrm{O}(1: 4), 40^{\circ} \mathrm{C},}{\stackrel{\mathrm{Na}_{2} \mathrm{~S}_{2} \mathrm{O}_{8} \text { (2.5 equiv.) }}{\longrightarrow}}$ $3 \mathrm{hrs}$.<smiles>CC1CC(C)[C@@H](CCS(=O)(=O)c2ccccc2)C(C=O)C1</smiles>
\begin{tabular}{l} 
Additive \\
\hline $\mathrm{Co}(\mathrm{acac})_{2} \times \mathrm{H}_{2} \mathrm{O}$ \\
$\mathrm{Co}(\mathrm{OAc})_{2} \times 4 \mathrm{H}_{2} \mathrm{O}$ \\
$\left.\mathrm{Co}(\mathrm{ClO})_{2}\right)_{2} \times 6 \mathrm{H}_{2} \mathrm{O}$ \\
$\mathrm{Mn}(\mathrm{OAC})_{2} \times 4 \mathrm{H}_{2} \mathrm{O}$ \\
$\mathrm{Fe}(\mathrm{OAc})_{2} \times \mathrm{H}_{2} \mathrm{O}$ \\
$\mathrm{CoCp}_{2}$ \\
$\quad \mathrm{PcCo}$ \\
$\mathrm{Co}(\mathrm{salen}) \times \mathrm{H}_{2} \mathrm{O}$ \\
Co(SO $\left.\mathrm{SO}_{4}\right) \times 7 \mathrm{H}_{2} \mathrm{O}$ \\
vitamin $\mathrm{B}_{12}$
\end{tabular} yield
$22 \%$
$27 \%$
$28 \%$
$24 \%$
$48 \%$
$24 \%$
$29 \%$
$7 \%$
$28 \%$
$2 \%$
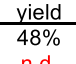
n.d. $47 \%$ $43 \%$
$63 \%$ $63 \%$

$60 \%$ $67 \%$ $71 \%$ [1.5 mmol] 


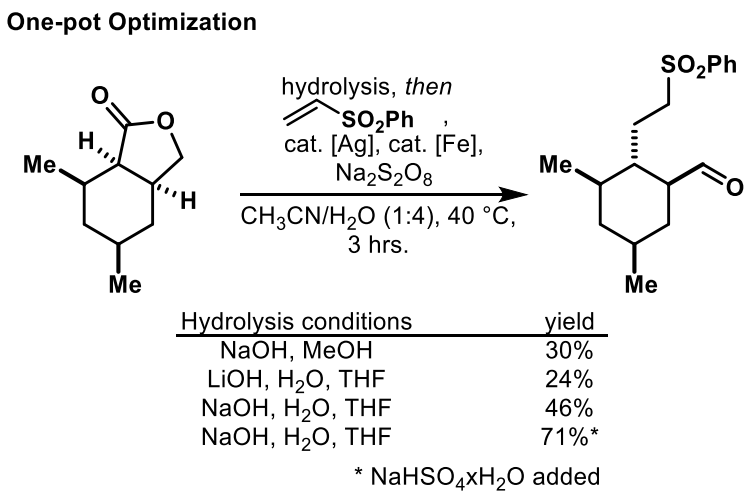

\section{Hydroxypyridone Formation (Step 9)}

Yields for reactions using model substrate were obtained by LCMS relative to 4 4'-di-tertbutylbiphenyl as internal standard (NMR calibration). All yields for reactions on maximiscin scaffold are isolated.
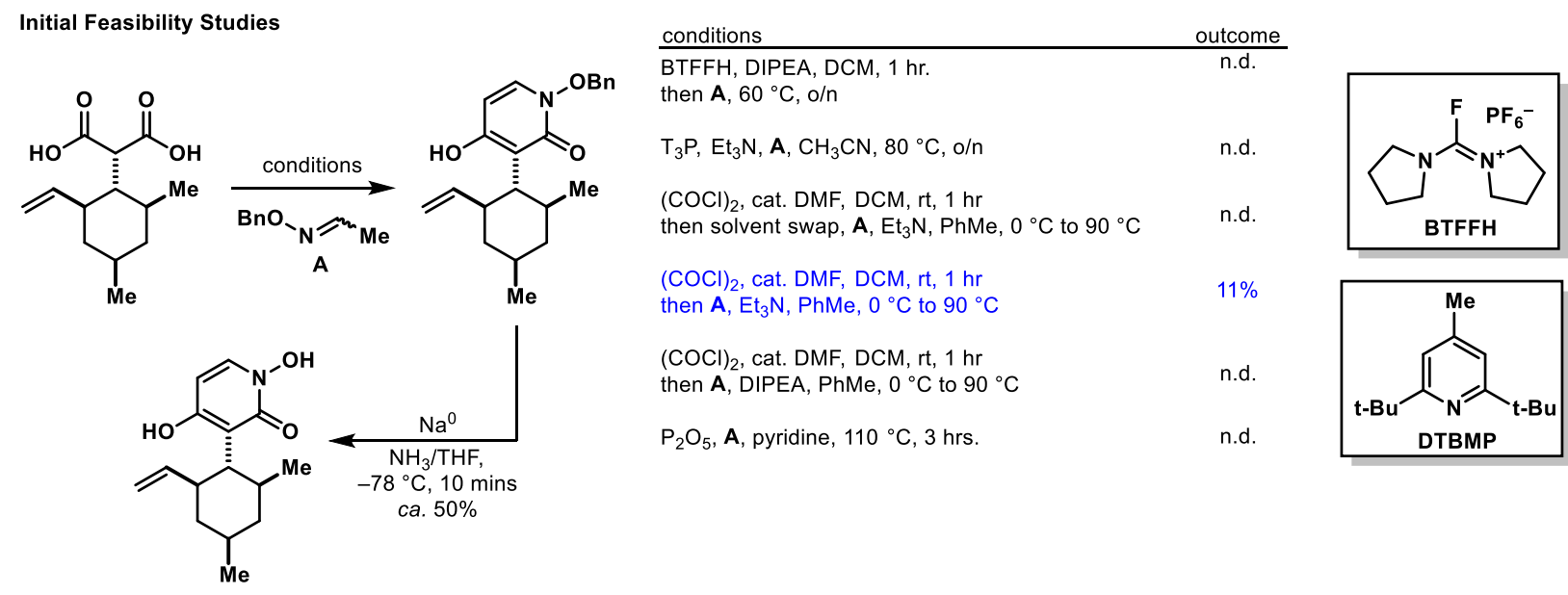

( \pm )-pyridoxatin

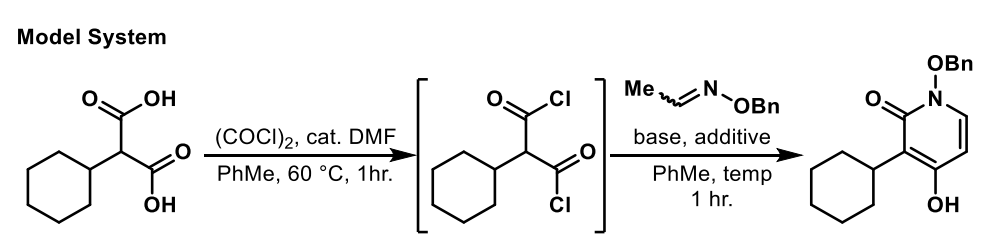

\begin{tabular}{cccc} 
conc. $(\mathrm{M})$ & base (eq.) & temperature $\left({ }^{\circ} \mathrm{C}\right)$ & yield $(\%)$ \\
\hline 0.1 & $\mathrm{Et}_{3} \mathrm{~N}(3)$ & 90 & $11 \%$ (isolated) \\
0.1 & none & 90 & no rxn (NMR) \\
0.05 & $\mathrm{Et}_{3} \mathrm{~N}(3)$ & 90 & $10 \%$ (NMR) \\
0.025 & $\mathrm{Et}_{3} \mathrm{~N}(3)$ & 90 & $8 \%$ (NMR) \\
0.1 & $\mathrm{Et}_{3} \mathrm{~N}(3)$ & $\mathrm{rt}$ & decomp. \\
0.1 & $\mathrm{Et}_{3} \mathrm{~N}(3)$ & 60 & trace \\
0.1 & $\mathrm{Et}_{3} \mathrm{~N}(4)$ & 90 & trace \\
0.1 & $\mathrm{Et}_{3} \mathrm{~N}(5)$ & 90 & decomp. \\
0.1 & $\mathrm{DTBMP}(3)+$ AgOTf & $-78{ }^{\circ} \mathrm{C}$ to rt & $10 \%$ (NMR)
\end{tabular}


Application to Maximiscin Scaffold<smiles>C=CC1CC(C)CC(C)[C@H]1C(C(=O)O)C(=O)O</smiles>
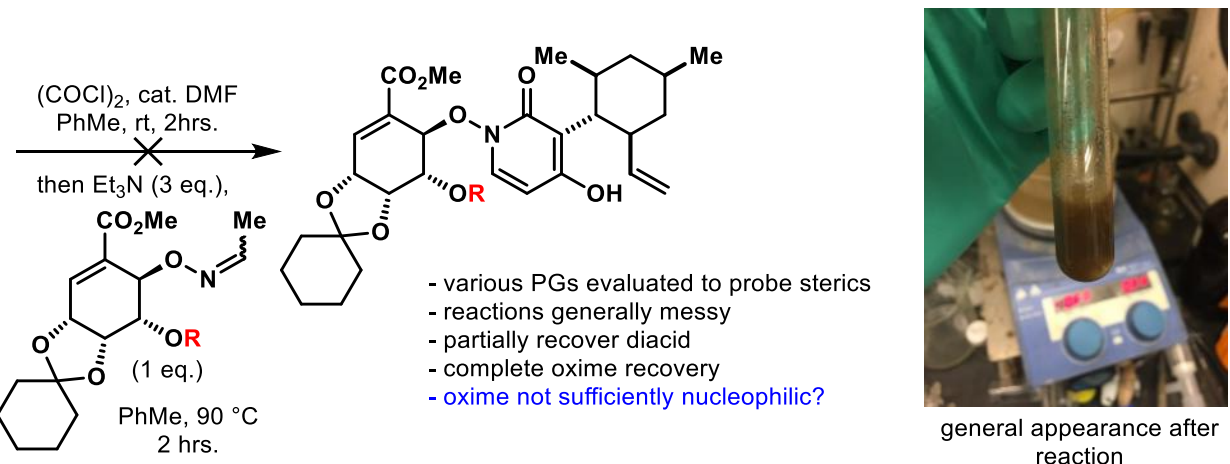

$\mathrm{R}=\mathrm{TBS}, \mathrm{MOM}, \mathrm{Ac}, \mathrm{H}$

Back to Model System - Understand Reactivity - what is the electrophile?<smiles>CC(C)CC(=O)C(C(=O)Cl)C1CCCCC1</smiles>
$1 \mathrm{hr}$.<smiles>O=C(Cl)C(C(=O)Cl)C1CCCCC1</smiles>

- not observed (NMR) no change in acid chloride:hydrolysis ratio
- enhance oxime nucleophilicity

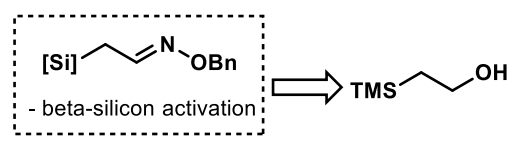

- TMS-oxime reactive at room temperature
- dual activation<smiles>O=C(Cl)C(C(=O)Cl)C1CCCCC1</smiles>

AgOTf, DTBMP

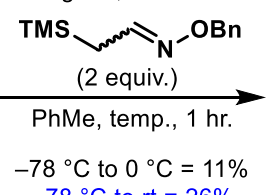
$-78{ }^{\circ} \mathrm{C}$ to $0{ }^{\circ} \mathrm{C}=11 \%$
$-78{ }^{\circ} \mathrm{C}$ to $\mathrm{rt}=26 \%$<smiles>CCCCn1ccc(O)c(C2CCCCC2)c1=O</smiles>

- TMS + Ag activation delivers highest yield
- substituted diacid chloride<smiles>C=CC1CC(C)CC(C)C1C(C(=O)Cl)C(=O)Cl</smiles><smiles>C=CC1CC(C)CC([N+](=O)[O-])[C@H]1c1c(O)ccn(OCCCC)c1=O</smiles>

- substituted oxime<smiles>O=C(Cl)C(C(=O)Cl)C1CCCCC1</smiles><smiles>[B-]O[C@@H]1C(O/N=C\C[W])C(C(=O)OC)=C[C@@H]2OC3(CCCCC3)O[C@H]21</smiles>

TMS

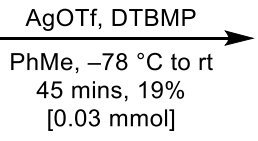<smiles>CO[C@H]1C(On2ccc(O)c(C3CCCCC3)c2=O)[C@H]([OH2+])C(C(C)=O)=C[C@H]2OC3(CCCCC3)O[C@H]21</smiles> 
Application to Maximsicin

note: unless otherwise stated, solvent swap was conducted after diacid chloride formation
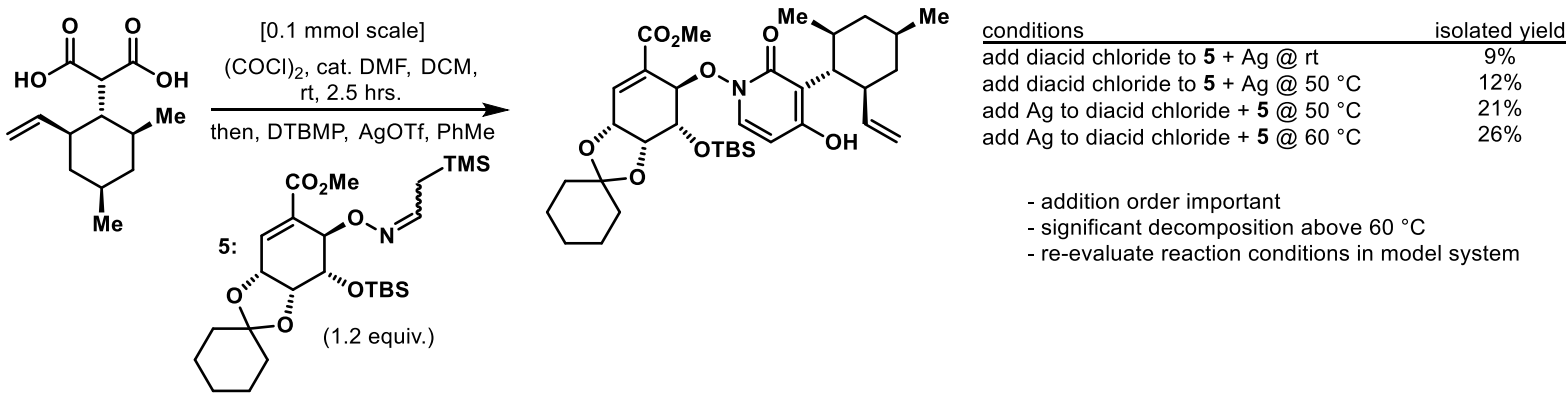

Solvent/Ag Effects

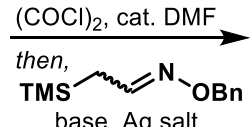

base, $\mathrm{Ag}$ salt

solvent, $60^{\circ} \mathrm{C}, 10 \mathrm{mins}$

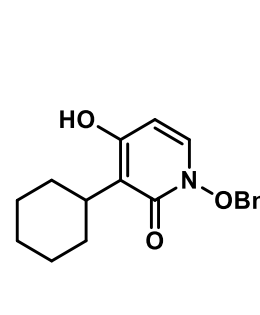

\begin{tabular}{|c|c|c|c|}
\hline \multicolumn{2}{|c|}{$\begin{array}{l}1.2 \text { equiv. oxime, } \\
2 \text { equiv. AgOTf }\end{array}$} & \multicolumn{2}{|c|}{$\begin{array}{l}1.2 \text { equiv. oxime, } \\
9: 1 \mathrm{MeNO}_{2} / \mathrm{PhMe}\end{array}$} \\
\hline solvent & yield & $\mathrm{Ag}^{+}$source & yield \\
\hline $\mathrm{PhMe}$ & $15 \%$ & AgOTf & $24 \%$ \\
\hline TFT & n.d. & $\mathrm{AgBF}_{4}$ & $10 \%$ \\
\hline $\mathrm{MeNO}_{2}$ & $26 \%$ & $\mathrm{AgSbF}_{6}$ & $23 \%$ \\
\hline DCE & $11 \%$ & $\mathrm{AgCN}$ & n.d. \\
\hline DCM & $11 \%$ & AgOTFA & $8 \%$ \\
\hline & & $\mathrm{AgNO}_{3}$ & n.d. \\
\hline ethane & & $\begin{array}{c}\mathrm{Ag}_{2} \mathrm{O} \\
\mathrm{AgF}\end{array}$ & $\begin{array}{l}\text { n.d. } \\
\text { n.d. }\end{array}$ \\
\hline
\end{tabular}

Application to Maximiscin
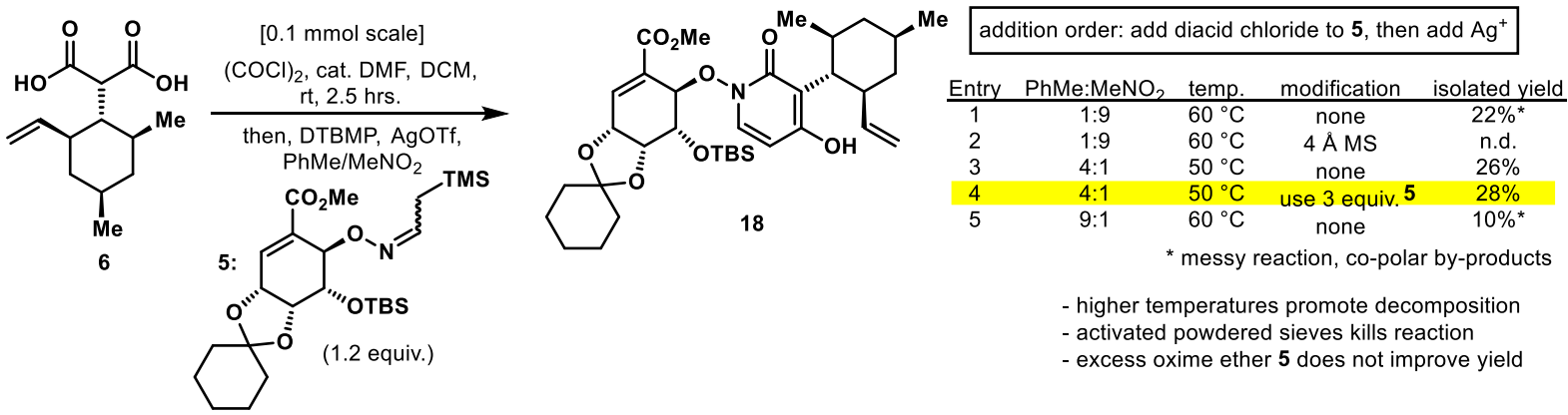

During the review process, it was suggested that silyl oxime 5 may react via initial $C-S i$ to $N$-Si transfer to form a transient silyl enamine. We do not believe a silyl enamine is generated under these reaction conditions. In nearly all cases, under Ag promoted conditions mass recovery of the silyl oxime $\mathbf{5}$ was excellent, after reaction workup. For example, entry 4 (see above) using a large

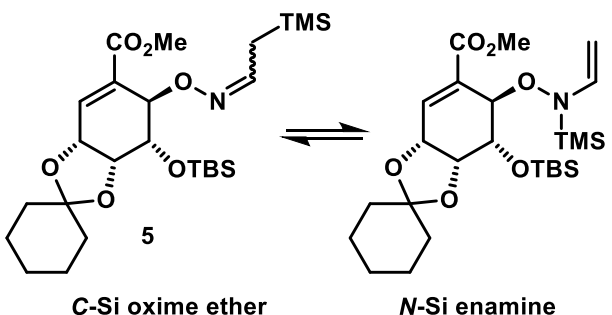
excess of oxime 5 ( $0.3 \mathrm{mmol}, 3$ equiv.) we obtained the product 18 in $28 \%$ yield $(0.028 \mathrm{mmol})$ with $95 \%(0.26 \mathrm{mmol})$ recovered $\mathbf{5}$. This result suggests that there is no silyl migration under productive reaction conditions, since the resulting silyl enamine would be quenched on aqueous workup to give des-TMS-5. The higher ratio of desTMS-5 to 5 observed under the final reaction conditions in $\mathrm{CH}_{3} \mathrm{CN}$ (vide infra) likely results from the harsher $\mathrm{MeOH}$ quench, which leads to desilylation of $\mathbf{5}$. 
Evaluate Other Lewis Acids

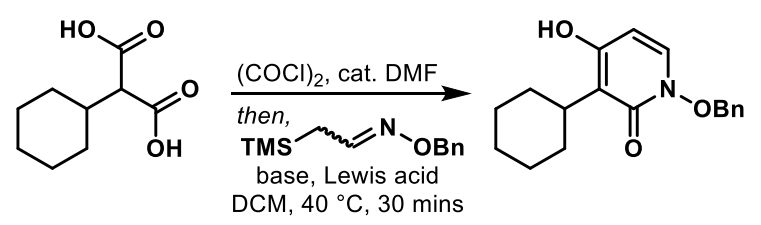

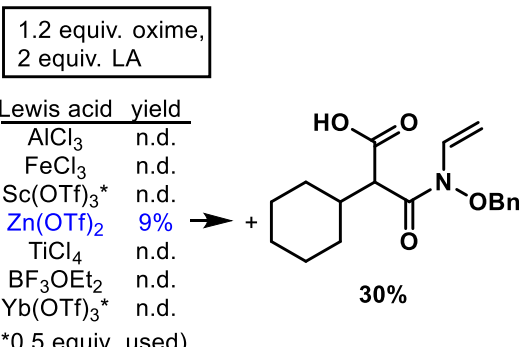

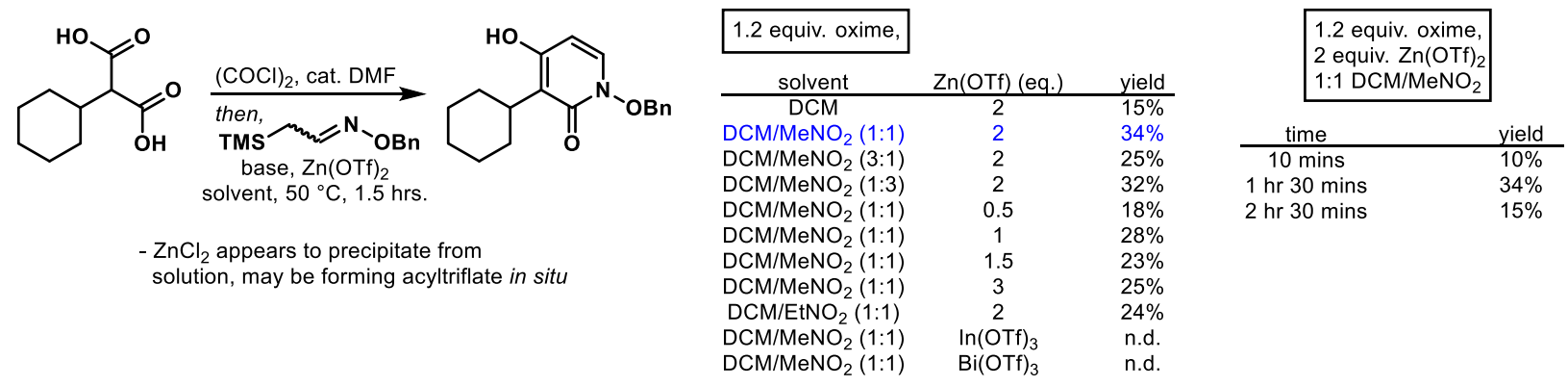

Results Do Not Translate to Maximiscin Scaffold
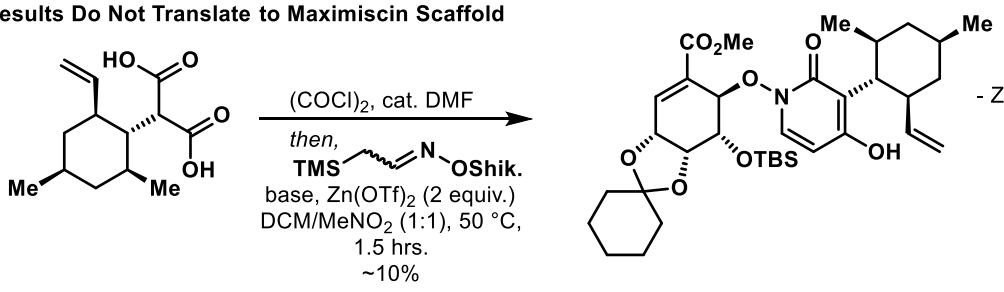

- Zn(OTf $)_{2}$ promoted significant decomposition and generated impurities which were co-polar with product

Back to model

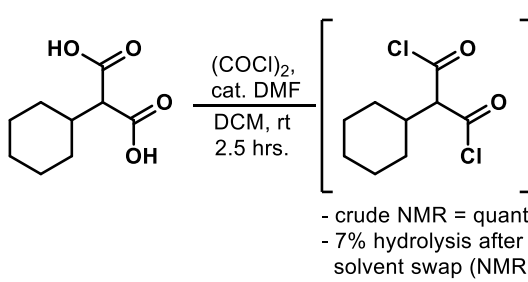

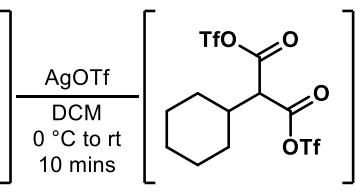

- $\mathrm{AgCl}$ precipitates from $\mathrm{r} \times$ - residual AgOTf insoluble - transfered as clear, yellow $\mathrm{CH}_{2} \mathrm{Cl}_{2}$ solution

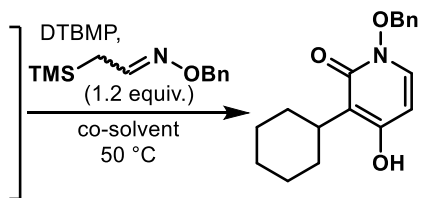

- acetonitirle optimal solven

- reaction likely proceeds via acyl triflate

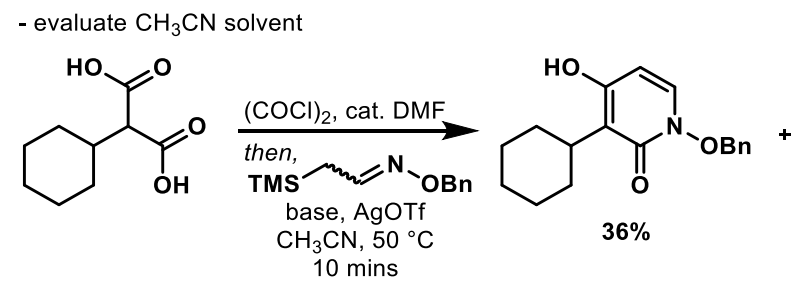<smiles>O=C(O)C(C(=O)Oc1ccn(OCCc2ccccc2)c(=O)c1C1CCCCC1)C1CCCCC1</smiles>

$23 \%$ 
- $\mathrm{MeOH}$ quench liberates product

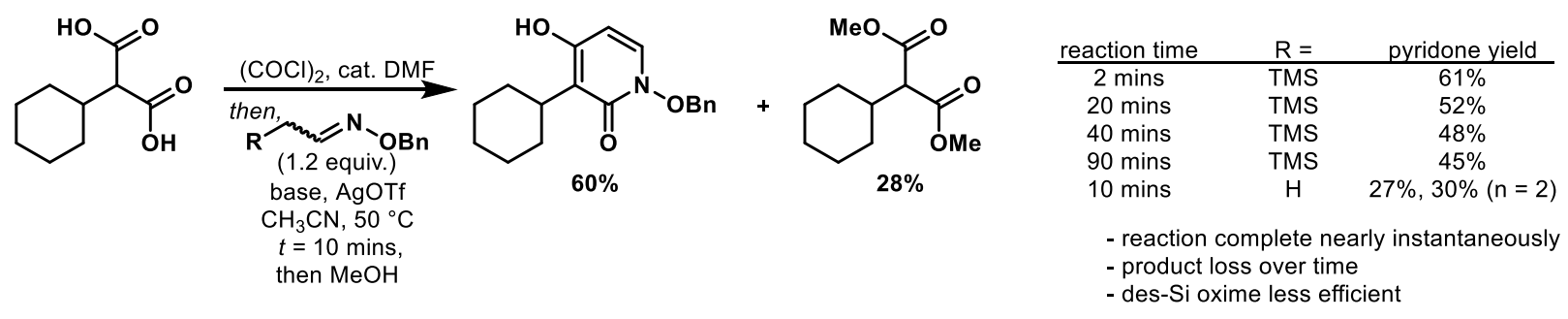

Application to Maximiscin<smiles>C=CC1CC(C)CC(C)[C@H]1C(C(=O)O)C(=O)O</smiles>

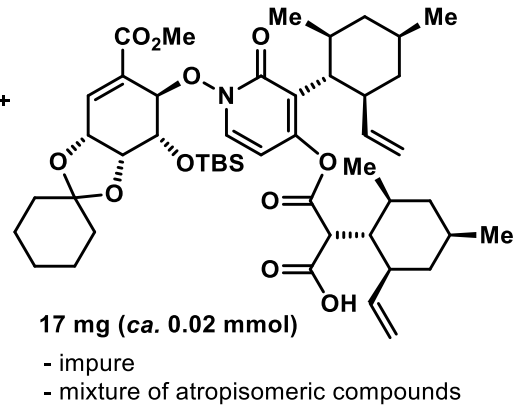

(1.2 equiv. $)$ 


\section{SYNTHETIC PROCEDURES AND CHARACTERIZATION DATA}

\section{General Synthesis of $\mathbf{C}-\mathbf{H}$ Activation Substrates}

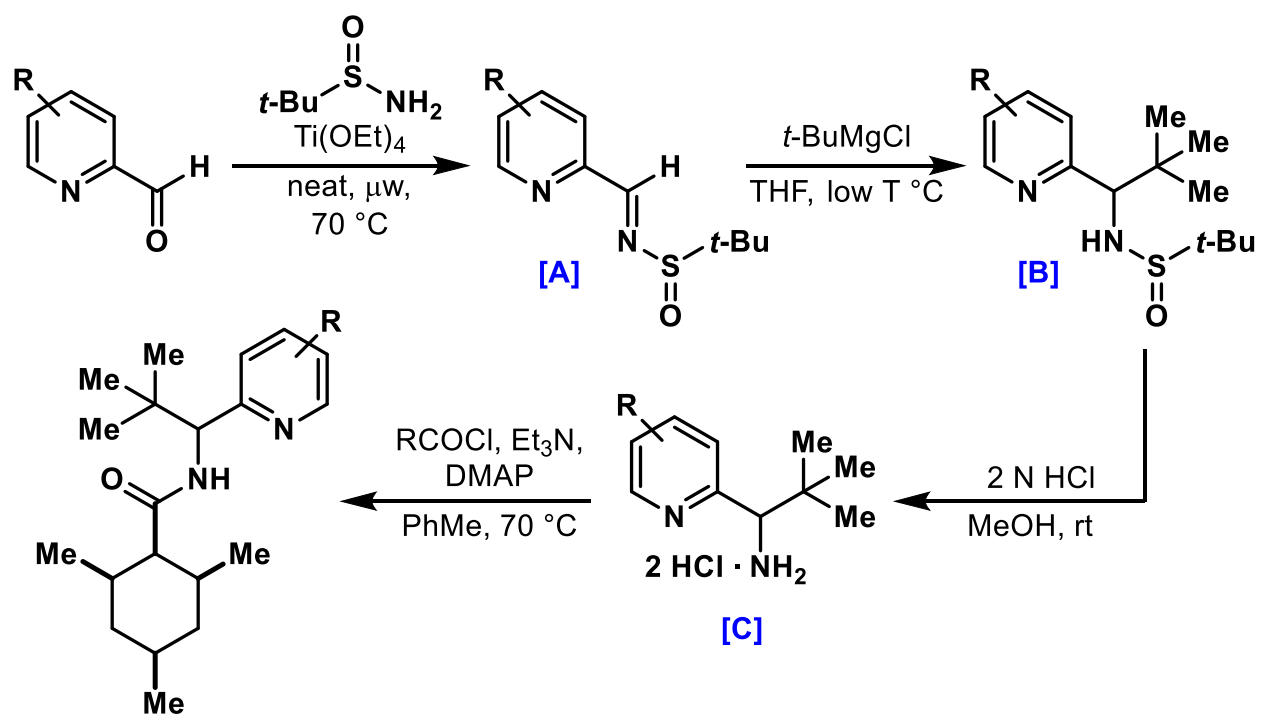

General procedure 1 (GP-1): Synthesis of $N$-sulfinylimines. $N$-Sulfinylimines were prepared using a modified literature procedure. ${ }^{6}$ To a $20 \mathrm{~mL}$ microwave vial equipped with egg-shaped stir bar was added the respective pyridinecarboxaldehyde (1 equiv.), $(S)-(-)-$ or $(R)-(+)-2$-methyl-2propanesulfinamide (1 equiv.) and titanium ethoxide (2 equiv.). The mixture was sealed under argon and allowed to react at $70{ }^{\circ} \mathrm{C}$ under microwave irradiation for 10-15 mins (Biotage Initiator). In the case of heterogeneous mixtures, a small volume of anhydrous $\mathrm{CH}_{2} \mathrm{Cl}_{2}$ could be added to facilitate reagent mixing. The reaction mixture was diluted with EtOAc $(5 \mathrm{~mL} / \mathrm{mmol})$ and added to a rapidly stirring solution of saturated aqueous sodium chloride $(0.3 \mathrm{~mL} / \mathrm{mmol})$. The resulting suspension was filtered over Celite ${ }^{\circledR}$, concentrated under reduced pressure and purified by column chromatography, if necessary.

General procedure 2 (GP-2): Synthesis of $N$-sulfinamides. $N$-Sulfinamides were prepared using a modified literature procedure. ${ }^{7}$ A solution of $N$-sulfinylimine ( 1 equiv.) in anhydrous THF $(0.3$ M) was cooled to $-78^{\circ} \mathrm{C}$ (unless otherwise specified). Tert-butyl magnesium chloride solution (1.5 equiv., $1 \mathrm{M}$ in THF) was added dropwise and the mixture was allowed to stir until complete by TLC ( $c a .1-3 \mathrm{hrs}$ ). The reaction mixture was quenched with saturated aqueous ammonium chloride and extracted three times with EtOAc. The combined organics were washed with brine, dried with sodium sulfate and concentrated in vacuo. The resulting syrup was purified by column chromatography.

General procedure 3 (GP-3): Synthesis of amine dihydrochlorides. Amine dihydrochlorides were prepared using a modified literature procedure. ${ }^{7}$ A solution of $N$-sulfinamide (1 equiv.) in anhydrous $\mathrm{MeOH}(0.3 \mathrm{M})$ was treated with $2 \mathrm{~N} \mathrm{HCl}^{\text {in }} \mathrm{Et}_{2} \mathrm{O}$ ( 3 equiv.) and the mixture was allowed to stir at room temperature for 3 hours. The reaction was concentrated under reduced pressure, and co-evaporated with toluene several times to yield a solid residue. The residue was triturated with $\mathrm{Et}_{2} \mathrm{O}$ and the suspension was poured over a fritted funnel. The solid was washed with two volumes 
of $\mathrm{Et}_{2} \mathrm{O}$ and quickly transferred to a vial (very hygroscopic). Solids were dried under high vacuum overnight before use in the next step.

General procedure 4 (GP-4): Synthesis of $\mathbf{C}-\mathbf{H}$ activation substrates. Carboxylic acid (1 equiv.) was treated with oxalyl chloride (1.2 equiv.) and $\mathrm{DMF}\left(0.05\right.$ equiv.) in $\mathrm{CH}_{2} \mathrm{Cl}_{2}(0.3 \mathrm{M})$ at room temperature. After 2 hours, solvent was removed in-vacuo (rotary evaporator in fume hood) and the yellow residue was re-suspended in anhydrous toluene $(1 \mathrm{M})$. A separate flame dried flask was charged with amine dihydrochloride (1 equiv.), DMAP (0.05 equiv.) and toluene (0.55 M). Triethylamine (3.7 equiv.) was added and the mixture was transferred to an oil bath preheated to $70{ }^{\circ} \mathrm{C}$. The acid chloride was added via cannula transfer, rinsing with toluene $(4 \mathrm{M})$ and the reaction was allowed to stir vigorously for 3 hours. The mixture was cooled to room temperature, diluted with $\mathrm{CH}_{2} \mathrm{Cl}_{2}$ and transferred to a separatory funnel. The organics were washed with saturated aqueous ammonium chloride and the aqueous layer was extracted twice with $\mathrm{CH}_{2} \mathrm{Cl}_{2}$. The combined organic extracts were washed with brine, dried with sodium sulfate and concentrated. The resulting oil was purified by column chromatography.

Analytical LCMS: The sample was analyzed on a Waters I-Class with a Waters Cortecs C18 column $(1.6 \mu \mathrm{m}, 2.1 \times 55 \mathrm{~mm})$ using a $0.1 \%$ aqueous formic acid:acetonitrile gradient $(0.8 \mathrm{~mL} / \mathrm{min}$, $10-99 \%$ acetonitrile over 2.5 minutes) at $35^{\circ} \mathrm{C}$. Compounds were detected by UV light (MaxPlot 220-400 nm) and identified by mass spec $\left(\mathrm{ESI}^{+}\right.$or $\left.\mathrm{ESI}^{-}\right)$.

Chiral SFC Analysis: The sample was analyzed on a Waters UPC2 SFC with a Daicel IG column (3 $\mu \mathrm{m}, 4.6 \times 250 \mathrm{~mm}$ ) under isocratic conditions $\left(4 \mathrm{~mL} / \mathrm{min}, 7 \% \mathrm{MeOH} / \mathrm{CO}_{2}, 1600\right.$ psi backpressure) at $30{ }^{\circ} \mathrm{C}$. The enantiomers were detected by UV light (260 nm).

\section{Compound 10a}<smiles>CC1CC(C)C(C(=O)NC(c2ccccn2)C(C)(C)C)C(C)C1</smiles>

$(S, E)-2-m e t h y l-N$-(pyridin-2-ylmethylene)propane-2-sulfinamide. [A]

Prepared according to GP-1 on $5.0 \mathrm{mmol}$ scale using (S)-(-)-2-methyl-2-propanesulfinamide. The product $(0.44 \mathrm{~g}, 2.07 \mathrm{mmol}, 41 \%$ yield $)$ was obtained after column chromatography (50\% EtOAc in hexanes)

${ }^{1}$ H NMR (600 MHz, CDCl 3$): \delta 8.68-8.64(\mathrm{~m}, 1 \mathrm{H}), 8.62(\mathrm{~s}, 1 \mathrm{H}), 7.95(\mathrm{~d}, J=7.9 \mathrm{~Hz}, 1 \mathrm{H}), 7.75$ $(\mathrm{td}, J=7.7,1.6 \mathrm{~Hz}, 1 \mathrm{H}), 7.33(\mathrm{ddd}, J=7.5,4.8,1.1 \mathrm{~Hz}, 1 \mathrm{H}), 1.21(\mathrm{~s}, 9 \mathrm{H})$. 
${ }^{13}$ C NMR (150 MHz, CDCl3): $\delta$ 163.2, 151.9, 149.7, 136.4, 125.46, 122.6, 57.6, 22.2.

HRMS (ESI-TOF): calculated for $\mathrm{C}_{10} \mathrm{H}_{15} \mathrm{~N}_{2} \mathrm{OS}[\mathrm{M}+\mathrm{H}]^{+}:$: 211.0900, found 211.0908.

(S)- $N$-((S)-2,2-dimethyl-1-(pyridin-2-yl)propyl)-2-methylpropane-2-sulfinamide.[B]

Prepared according to GP-2 on $2.1 \mathrm{mmol}$ scale. The product was obtained after column chromatography $(60 \rightarrow 80 \%$ EtOAc in hexanes) as a 9:1 mixture of diastereomers favoring the desired compound ( $0.50 \mathrm{~g}, 1.88 \mathrm{mmol}, 91 \%$ yield).

${ }^{1} \mathbf{H}$ NMR (400 MHz, CDCl $) \delta 8.54(\mathrm{~d}, J=4.4 \mathrm{~Hz}, 1 \mathrm{H}), 7.59(\mathrm{t}, J=7.9 \mathrm{~Hz}, 1 \mathrm{H}), 7.23-7.09(\mathrm{~m}$, 2H), 4.05 (d, $J=7.9 \mathrm{~Hz}, 1 \mathrm{H}), 1.29$ (s, 9H), $0.91(\mathrm{~s}, 9 \mathrm{H})$.

${ }^{13}$ C NMR (150 MHz, CDCl3) $\delta$ 159.5, 148.7, 135.7, 124.0, 122.3, 68.7, 56.3, 36.6, 26.8, 23.2.

HRMS (ESI-TOF): calculated for $\mathrm{C}_{14} \mathrm{H}_{25} \mathrm{~N}_{2} \mathrm{OS}[\mathrm{M}+\mathrm{H}]^{+}: 269.1682$, found 269.1691 .

(S)-2,2-dimethyl-1-(pyridin-2-yl)propan-1-amine dihydrochloride. [C]

Prepared according to GP-3 on $1.88 \mathrm{mmol}$ scale. The product was obtained after filtration as an orange solid ( $0.247 \mathrm{~g}, 1.05 \mathrm{mmol}, 56 \%$ yield).

${ }^{1}$ H NMR (400 MHz, DMSO-d $) \delta 8.66(\mathrm{~d}, J=4.3 \mathrm{~Hz}, 1 \mathrm{H}), 8.46(\mathrm{~s}, 3 \mathrm{H}), 7.93(\mathrm{t}, J=7.3 \mathrm{~Hz}, 1 \mathrm{H})$, $7.55(\mathrm{~d}, J=7.7 \mathrm{~Hz}, 1 \mathrm{H}), 7.47$ (dd, $J=7.2,5.1 \mathrm{~Hz}, 1 \mathrm{H}), 4.26(\mathrm{q}, J=5.4 \mathrm{~Hz}, 1 \mathrm{H}), 0.94(\mathrm{~s}, 9 \mathrm{H})$.

${ }^{13}$ C NMR (150 MHz, DMSO-d 6 ) $\delta$ 154.0, 147.2, 138.4, 124.8, 124.2, 61.9, 34.2, 26.2.

HRMS (ESI-TOF): calculated for $\mathrm{C}_{10} \mathrm{H}_{17} \mathrm{~N}_{2}[\mathrm{M}+\mathrm{H}]^{+}$: 165.1386 , found 165.1391 .

$N$-((S)-2,2-dimethyl-1-(pyridin-2-yl)propyl)-2,4,6-trimethylcyclohexane-1-carboxamide 10a. Prepared according to GP-4 on 1.00 mmol scale. Purified by column chromatography $(10 \rightarrow 15 \%$ EtOAc in hexanes) to afford $0.24 \mathrm{~g}(76 \%)$ of the title compound 10a.

Physical state: white crystalline solid

TLC: $\mathrm{R}_{f}=0.5$ (20\% EtOAc in hexanes; visualization by UV, $\left.\mathrm{KMnO}_{4}\right)$

m.p.: $68-70{ }^{\circ} \mathrm{C}$

${ }^{1} \mathbf{H}$ NMR $\left(600 \mathrm{MHz}, \mathbf{C D C l}_{3}\right): \delta 8.50(\mathrm{~d}, J=4.7 \mathrm{~Hz}, 1 \mathrm{H}), 7.56(\mathrm{td}, J=7.6,1.8 \mathrm{~Hz}, 1 \mathrm{H}), 7.15(\mathrm{~d}, J$ $=7.8 \mathrm{~Hz}, 1 \mathrm{H}), 7.13(\mathrm{dd}, J=7.5,4.9 \mathrm{~Hz}, 1 \mathrm{H}), 6.85(\mathrm{~d}, J=8.9 \mathrm{~Hz}, 1 \mathrm{H}), 4.88(\mathrm{~d}, J=9.2 \mathrm{~Hz}, 1 \mathrm{H})$, $2.21(\mathrm{t}, J=4.7 \mathrm{~Hz}, 1 \mathrm{H}), 1.71-1.60(\mathrm{~m}, 2 \mathrm{H}), 1.58(\mathrm{q}, J=12.3 \mathrm{~Hz}, 1 \mathrm{H}), 1.43-1.27(\mathrm{~m}, 3 \mathrm{H}), 1.25$ $-1.19(\mathrm{~m}, 1 \mathrm{H}), 1.02(\mathrm{~d}, \mathrm{~J}=6.8 \mathrm{~Hz}, 3 \mathrm{H}), 0.91(\mathrm{~s}, 9 \mathrm{H}), 0.89(\mathrm{~d}, \mathrm{~J}=6.3 \mathrm{~Hz}, 3 \mathrm{H}), 0.57(\mathrm{~d}, J=7.0 \mathrm{~Hz}$, $3 \mathrm{H})$.

${ }^{13}$ C NMR (125 MHz, $\left.\mathbf{C D C l}_{3}\right): \delta$ 173.2, 159.2, 148.6, 135.6, 124.3, 122.1, 60.7, 53.4, 37.8, 37.8, $35.7,35.3,35.1,32.7,27.0,22.5,20.4,19.8$. 
HRMS (m/z): calculated for $\mathrm{C}_{20} \mathrm{H}_{33} \mathrm{~N}_{2} \mathrm{O}[\mathrm{M}+\mathrm{H}]^{+}: 317.2587$, found 317.2597.

Analytical LCMS:

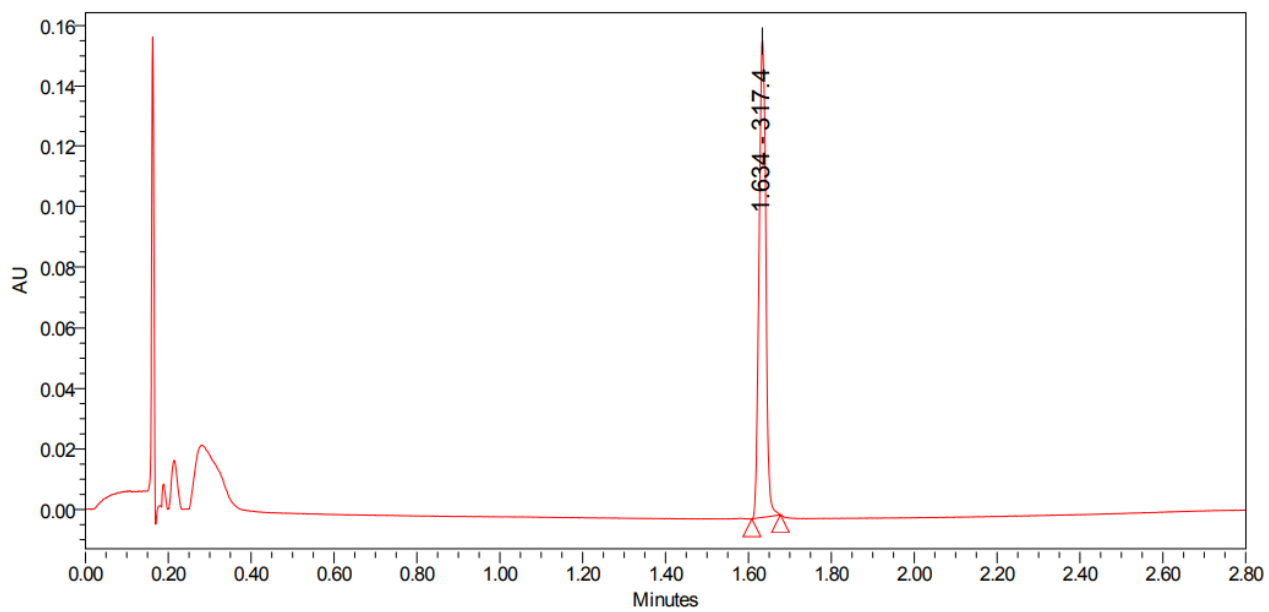

Chiral SFC: $($ Ent $1=9.4 \%$, Ent $2=90.6 \%)$

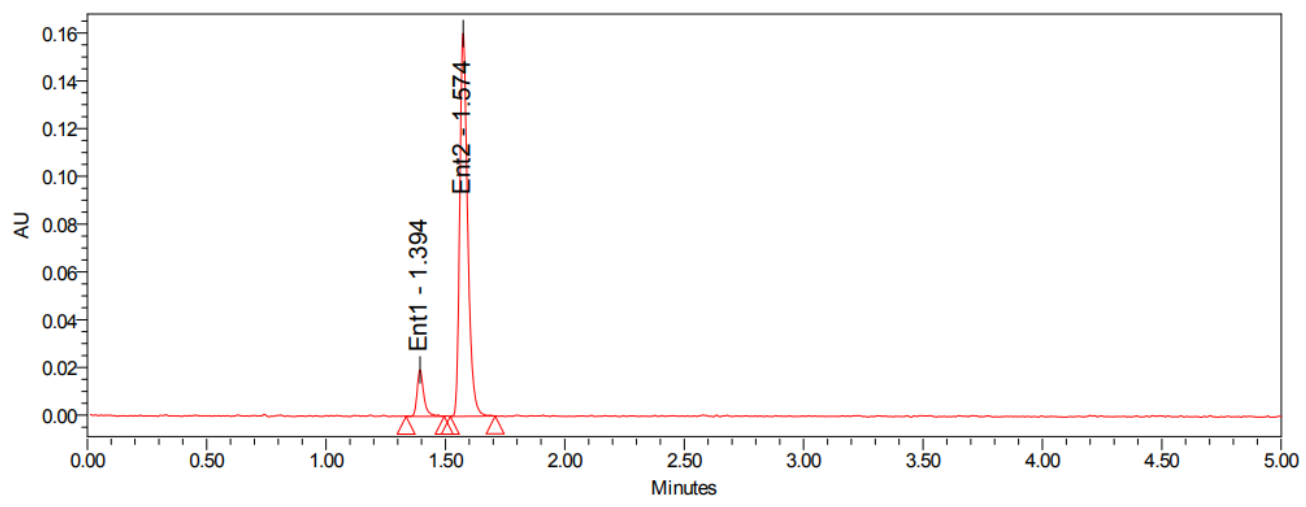

\section{Compound 10b}<smiles>COc1cccc(C(NC(=O)C2C(C)CC(C)CC2C)C(C)(C)C)n1</smiles>

$10 b$

(S,E)- $N$-((6-methoxypyridin-2-yl)methylene)-2-methylpropane-2-sulfinamide. [A]

Prepared according to GP-1 on $7.30 \mathrm{mmol}$ scale using $(S)$-(-)-2-methyl-2-propanesulfinamide. The product (1.43 g, $6.81 \mathrm{mmol}, 93 \%$ yield) was sufficiently pure to be used in the next step. 
${ }^{1} \mathbf{H}$ NMR (600 MHz, CDCl 3$): \delta 8.59(\mathrm{~s}, 1 \mathrm{H}), 7.66(\mathrm{t}, J=7.7 \mathrm{~Hz}, 1 \mathrm{H}), 7.61(\mathrm{dd}, J=7.3,0.8 \mathrm{~Hz}$, $1 \mathrm{H}), 6.85(\mathrm{dd}, J=8.1,0.9 \mathrm{~Hz}, 1 \mathrm{H}), 3.98(\mathrm{~s}, 3 \mathrm{H}), 1.28(\mathrm{~s}, 9 \mathrm{H})$.

${ }^{13}$ C NMR (150 MHz, CDCl3): $\delta$ 164.3, 163.9, 150.2, 139.0, 116.1, 114.1, 58.2, 53.7, 22.8.

HRMS (ESI-TOF): calculated for $\mathrm{C}_{11} \mathrm{H}_{17} \mathrm{~N}_{2} \mathrm{O}_{2} \mathrm{~S}[\mathrm{M}+\mathrm{H}]^{+}:$: 241.1005, found 241.1011.

(S)-N-((S)-1-(6-methoxypyridin-2-yl)-2,2-dimethylpropyl)-2-methylpropane-2-sulfinamide.

[B]

Prepared according to GP-2 on $6.41 \mathrm{mmol}$ scale. The product was obtained after column chromatography $(40 \rightarrow 60 \%$ EtOAc in hexanes) as a $4: 1$ mixture of diastereomers favoring the desired compound (0.95 g, $3.19 \mathrm{mmol}, 50 \%$ yield).

${ }^{1}$ H NMR (600 MHz, CDCl $): \delta 7.47(\mathrm{dd}, J=8.2,7.3 \mathrm{~Hz}, 1 \mathrm{H}), 6.75(\mathrm{~d}, J=7.2 \mathrm{~Hz}, 1 \mathrm{H}), 6.59$ (d, $J=8.0 \mathrm{~Hz}, 1 \mathrm{H}), 5.20(\mathrm{~d}, J=7.9 \mathrm{~Hz}, 1 \mathrm{H}), 3.95(\mathrm{~d}, J=8.0 \mathrm{~Hz}, 1 \mathrm{H}), 3.89$ (s, 3H), $1.28(\mathrm{~s}, 9 \mathrm{H}), 0.91$ $(\mathrm{s}, 9 \mathrm{H})$.

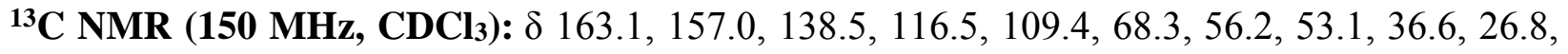
23.1 .

HRMS (ESI-TOF): calculated for $\mathrm{C}_{15} \mathrm{H}_{27} \mathrm{~N}_{2} \mathrm{O}_{2} \mathrm{~S}[\mathrm{M}+\mathrm{H}]^{+}:$: 299.1788, found 299.1798.

(S)-1-(6-methoxypyridin-2-yl)-2,2-dimethylpropan-1-amine dihydrochloride. [C]

Prepared according to GP-3 on $3.19 \mathrm{mmol}$ scale. The product was obtained after filtration as an off-white solid (0.72 g, $2.69 \mathrm{mmol}, 84 \%$ yield).

${ }^{1}$ H NMR (500 MHz, DMSO-d6): $\delta 8.35$ (s, 3H), $7.72(\mathrm{~d}, J=8.0 \mathrm{~Hz}, 1 \mathrm{H}), 7.03$ (d, $J=7.2 \mathrm{~Hz}$, $1 \mathrm{H}), 6.80(\mathrm{~d}, J=8.3 \mathrm{~Hz}, 1 \mathrm{H}), 4.10(\mathrm{q}, J=4.9,4.3 \mathrm{~Hz}, 1 \mathrm{H}), 3.91$ (s, 3H), 0.97 (s, 9H).

${ }^{13}$ C (151 MHz, DMSO-d6): $\delta$ 162.8, 152.6, 139.3, 117.1, 110.0, 62.1, 53.5, 34.0, 26.3.

HRMS (ESI-TOF): calculated for $\mathrm{C}_{11} \mathrm{H}_{19} \mathrm{~N}_{2} \mathrm{O}^{+}[\mathrm{M}+\mathrm{H}]^{+}:$195.1492, found 195.1484.

(N-(S)-1-(6-methoxypyridin-2-yl)-2,2-dimethylpropyl)-2,4,6-trimethylcyclohexane-1-

carboxamide 10b. Prepared according to GP-4 on $1.50 \mathrm{mmol}$ scale. The product was purified by column chromatography $(10 \rightarrow 15 \%$ EtOAc in hexanes $)$ to afford $0.31 \mathrm{~g}(60 \%$ yield $)$ of the title compound 10b.

Physical state: white crystalline solid

TLC: $\mathrm{R}_{f}=0.65$ (20\% EtOAc in hexanes; visualization by $\left.\mathrm{UV}, \mathrm{KMnO}_{4}\right)$

m.p.: $69-71{ }^{\circ} \mathrm{C}$

${ }^{1}$ H NMR (400 MHz, CDCl $): \delta 7.47(\mathrm{dd}, J=8.3,7.2 \mathrm{~Hz}, 1 \mathrm{H}), 6.75(\mathrm{~d}, J=6.9 \mathrm{~Hz}, 1 \mathrm{H}), 6.63$, (brs, $1 \mathrm{H}), 6.59(\mathrm{dd}, J=8.3,0.6 \mathrm{~Hz}, 1 \mathrm{H}), 4.81(\mathrm{~d}, J=9.4 \mathrm{~Hz}, 1 \mathrm{H}), 3.91(\mathrm{~s}, 3 \mathrm{H}), 2.19(\mathrm{t}, J=4.5 \mathrm{~Hz}, 1 \mathrm{H})$, $1.73-1.55(\mathrm{~m}, 3 \mathrm{H}), 1.46-1.17(\mathrm{~m}, 4 \mathrm{H}), 1.02(\mathrm{~d}, J=6.6 \mathrm{~Hz}, 3 \mathrm{H}), 0.93(\mathrm{~s}, 9 \mathrm{H}), 0.90(\mathrm{~d}, J=6.1$ $\mathrm{Hz}, 3 \mathrm{H}), 0.62$ (d, J = 7.0 Hz, 3H). 


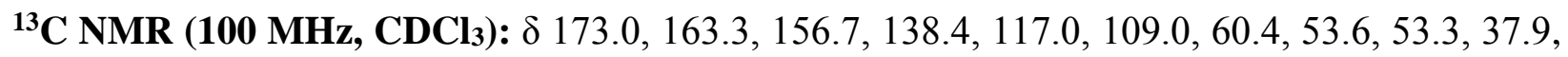
$37.7,35.6,35.4,35.1,32.7,27.1,22.5,20.3,20.1$.

HRMS (ESI-TOF): calculated for $\mathrm{C}_{21} \mathrm{H}_{34} \mathrm{~N}_{2} \mathrm{NaO}_{2}$ [M+Na] $]^{+}: 369.2512$, found 369.2518. Analytical LCMS:

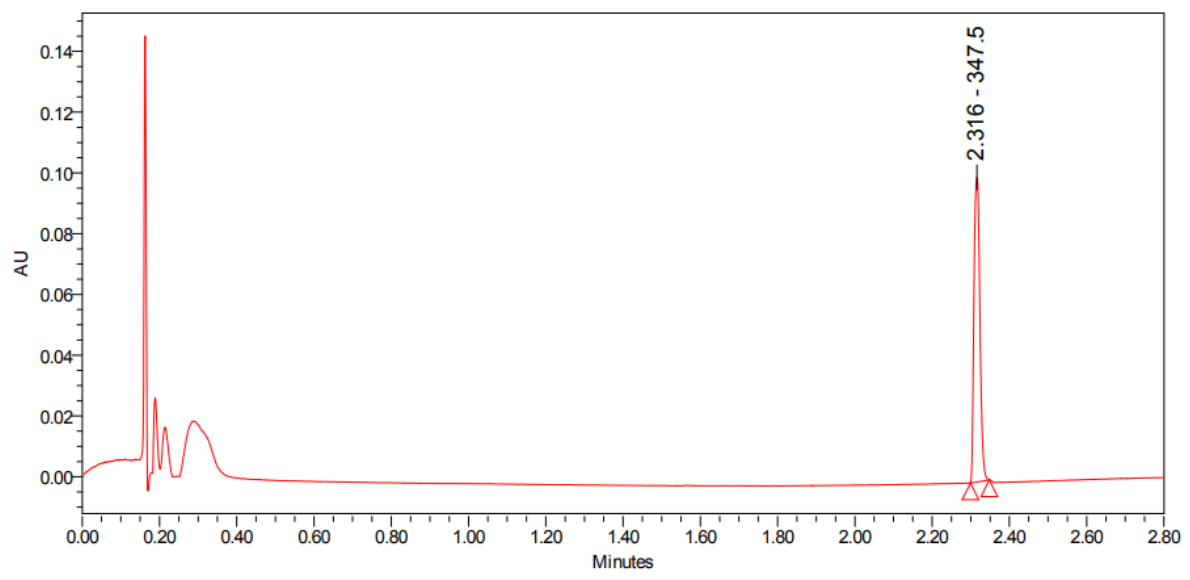

Chiral SFC: $($ Ent $1=16.3 \%$, Ent $2=83.7 \%)$

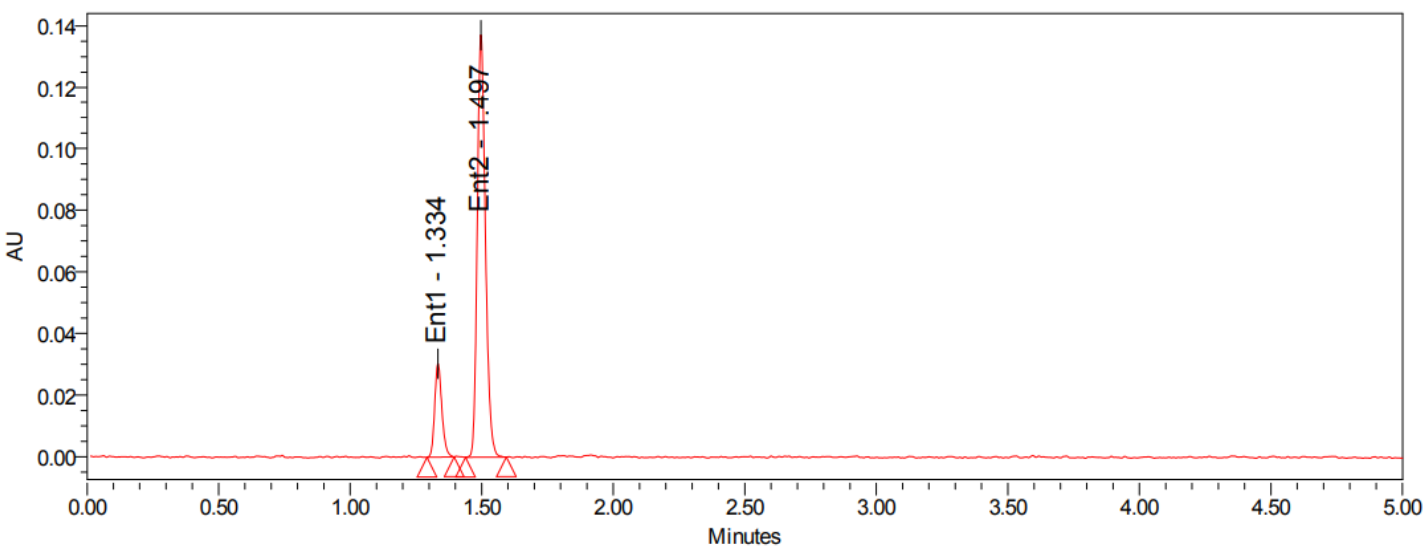




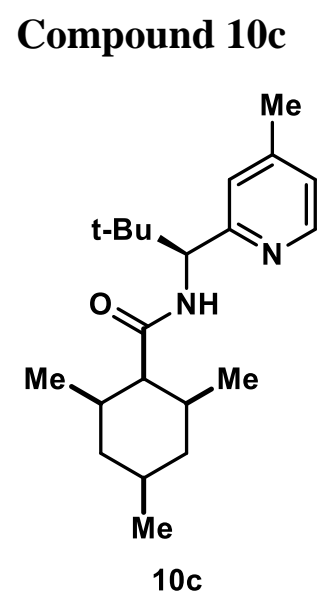

(S,E)-2-methyl- $N$-((4-methylpyridin-2-yl)methylene)propane-2-sulfinamide. [A]

Prepared according to GP-1 on $5.30 \mathrm{mmol}$ scale using $(S)$-(-)-2-methyl-2-propanesulfinamide. The product $(0.50 \mathrm{~g}, 2.01 \mathrm{mmol}, 42 \%$ yield $)$ was obtained after purification by column chromatography $(40 \rightarrow 50 \%$ EtOAc in hexanes).

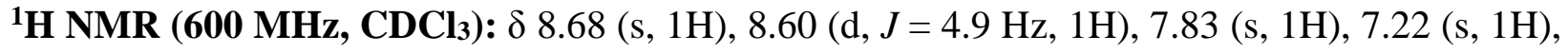
$2.44(\mathrm{~s}, 3 \mathrm{H}), 1.29$ (s, 9H).

${ }^{13}$ C NMR (150 MHz, CDCl3): $\delta$ 164.1, 152.5, 150.1, 148.3, 127.0, 124.0, 58.2, 22.9, 21.2.

HRMS (ESI-TOF): calculated for $\mathrm{C}_{11} \mathrm{H}_{17} \mathrm{~N}_{2} \mathrm{OS}^{+}[\mathrm{M}+\mathrm{H}]^{+} 225.1056$, found 225.1064.

(S)-N-((S)-2,2-dimethyl-1-(4-methylpyridin-2-yl)propyl)-2-methylpropane-2-sulfinamide.

[B]

Prepared according to GP-2 on 2.01 mmol scale. The product was obtained after column chromatography $(40 \rightarrow 60 \%$ EtOAc in hexanes) as a $10: 1$ mixture of diastereomers favoring the desired compound $(0.22 \mathrm{~g}, 0.78 \mathrm{mmol}, 39 \%$ yield).

${ }^{1}$ H NMR (500 MHz, CDCl $): \delta 8.38(\mathrm{~d}, J=5.0 \mathrm{~Hz}, 1 \mathrm{H}), 6.98(\mathrm{~s}, 1 \mathrm{H}), 6.95(\mathrm{~d}, J=4.7 \mathrm{~Hz}, 1 \mathrm{H})$, $5.27(\mathrm{~d}, J=7.6 \mathrm{~Hz}, 1 \mathrm{H}), 3.98(\mathrm{~d}, J=7.7 \mathrm{~Hz}, 1 \mathrm{H}), 2.31(\mathrm{~s}, 3 \mathrm{H}), 1.28(\mathrm{~s}, 9 \mathrm{H}), 0.90(\mathrm{~s}, 9 \mathrm{H})$.

${ }^{13}$ C NMR (125 MHz, CDCl 3$): \delta 159.4,148.4,146.8,124.9,123.3,68.8,56.3,36.6,26.9,23.2$, 21.2 .

HRMS (ESI-TOF): calculated for $\mathrm{C}_{15} \mathrm{H}_{27} \mathrm{~N}_{2} \mathrm{OS}[\mathrm{M}+\mathrm{H}]^{+}: 283.1839$, found 283.1834.

(S)-2,2-dimethyl-1-(4-methylpyridin-2-yl)propan-1-amine dihydrochloride. [C]

Prepared according to GP-3 on $0.78 \mathrm{mmol}$ scale. The product was obtained after filtration as an off-white solid ( $0.188 \mathrm{~g}, 0.75 \mathrm{mmol}, 96 \%$ yield).

${ }^{1}$ H NMR (500 MHz, DMSO-d6) $\delta 8.51(\mathrm{~d}, J=5.0 \mathrm{~Hz}, 1 \mathrm{H}), 8.34(\mathrm{~s}, 3 \mathrm{H}), 7.36(\mathrm{~s}, 1 \mathrm{H}), 7.31(\mathrm{~d}, J$ $=4.4 \mathrm{~Hz}, 1 \mathrm{H}), 4.18(\mathrm{~d}, J=5.4 \mathrm{~Hz}, 1 \mathrm{H}), 2.37(\mathrm{~s}, 3 \mathrm{H}), 0.94(\mathrm{~s}, 9 \mathrm{H})$.

${ }^{13}$ C NMR (500 MHz, DMSO-d6) $\delta$ 128.9, 128.2, 125.3, 125.2, 124.5, 62.1, 34.0, 26.2, 20.6. 
HRMS (ESI-TOF): calculated for $\mathrm{C}_{11} \mathrm{H}_{19} \mathrm{~N}_{2}[\mathrm{M}+\mathrm{H}]^{+}: 179.1543$, found 179.1541 .

(N-(S)-2,2-dimethyl-1-(4-methylpyridin-2-yl)propyl)-2,4,6-trimethylcyclohexane-1carboxamide 10c. Prepared according to GP-4 on $0.75 \mathrm{mmol}$ scale. The product was purified by column chromatography $(10 \rightarrow 15 \%$ EtOAc in hexanes) to afford the title compound 10c $(0.160 \mathrm{~g}$, $0.48 \mathrm{mmol}, 65 \%$ yield).

Physical state: white crystalline solid.

TLC: $\mathrm{R}_{f}=0.5\left(20 \%\right.$ EtOAc in hexanes, visualization $\left.\mathrm{UV}, \mathrm{KMnO}_{4}\right)$

m.p.: $55-57^{\circ} \mathrm{C}$

${ }^{1} \mathbf{H}$ NMR (400 MHz, CDCl $): \delta 8.32(\mathrm{~d}, J=4.7 \mathrm{~Hz}, 1 \mathrm{H}), 6.95(\mathrm{~s}, 1 \mathrm{H}), 6.92(\mathrm{~d}, J=4.8 \mathrm{~Hz}, 1 \mathrm{H})$, $6.84(\mathrm{~d}, J=9.0 \mathrm{~Hz}, 1 \mathrm{H}), 4.80(\mathrm{~d}, J=9.3 \mathrm{~Hz}, 1 \mathrm{H}), 2.29$ (s, 3H), 2.19 (t, $J=4.3 \mathrm{~Hz}, 1 \mathrm{H}), 1.71-$ $1.46(\mathrm{~m}, 3 \mathrm{H}), 1.40-1.29(\mathrm{~m}, 3 \mathrm{H}), 1.21(\mathrm{~d}, J=12.2 \mathrm{~Hz}, 1 \mathrm{H}), 1.00(\mathrm{~d}, J=6.4 \mathrm{~Hz}, 3 \mathrm{H}), 0.89$ (s, $9 \mathrm{H}), 0.87(\mathrm{~d}, J=5.2 \mathrm{~Hz}, 3 \mathrm{H}), 0.56(\mathrm{~d}, J=6.8 \mathrm{~Hz}, 3 \mathrm{H})$.

${ }^{13}$ C NMR (125 MHz, CDCl 3$): ~ \delta 173.3,158.9,148.3,146.7,125.2,123.1,60.6,53.3,37.8,37.8$, 35.6, 35.3, 35.1, 32.7, 27.0, 22.5, 21.1, 20.4, 19.8.

HRMS (ESI-TOF): calculated for $\mathrm{C}_{21} \mathrm{H}_{34} \mathrm{~N}_{2} \mathrm{NaO}[\mathrm{M}+\mathrm{Na}]^{+}: 353.2563$, found 353.2570. Analytical LCMS:

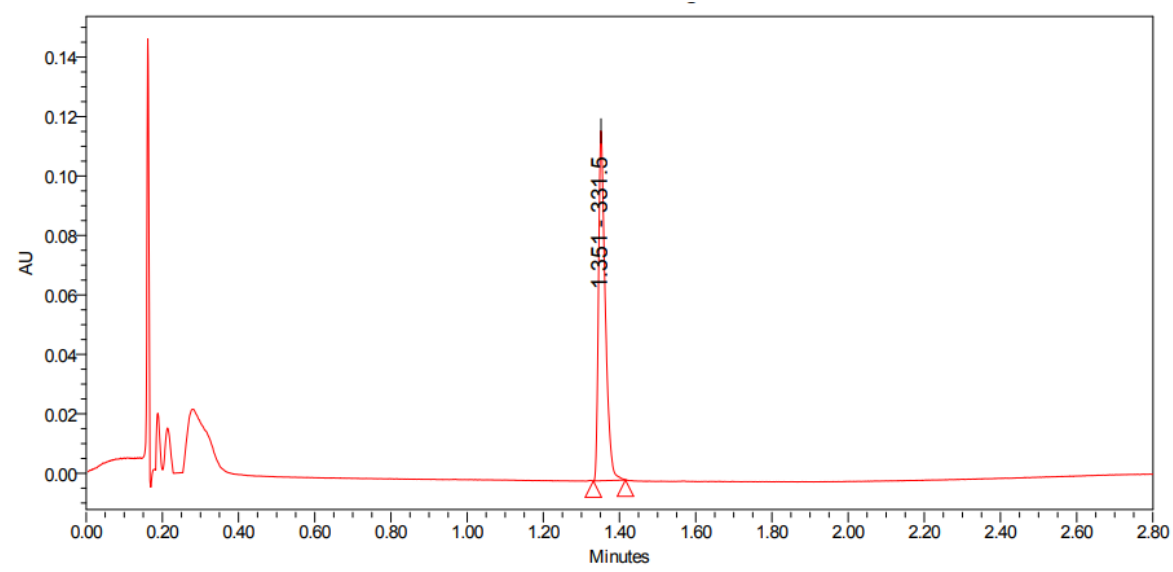


Chiral SFC: $($ Ent $1=6.4 \%$, Ent $2=93.6 \%)$

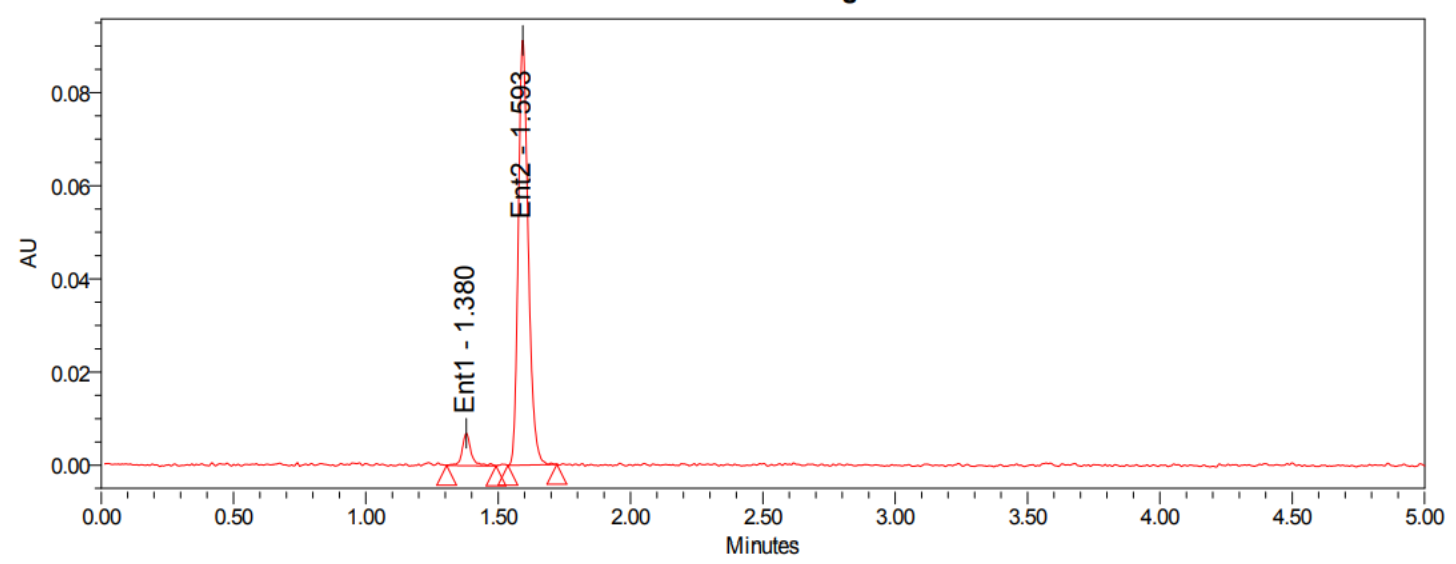

Compound SI-3.<smiles>CC1CC(C)C(C(=O)NC(c2cc(Cl)ccn2)C(C)(C)C)C(C)C1</smiles>

$N$-((S)-1-(4-chloropyridin-2-yl)-2,2-dimethylpropyl)-2,4,6-trimethylcyclohexane-1carboxamide. For preparation and spectral data, see compound 10d.

\section{Compound 10e}<smiles>CC1CC(C)C(C(=O)NC(c2ccc(Cl)cn2)C(C)(C)C)C(C)C1</smiles>

(S,E)-N-((5-chloropyridin-2-yl)methylene)-2-methylpropane-2-sulfinamide. [A] 
Prepared according to GP-1 on $7.10 \mathrm{mmol}$ scale using $(S)$-(-)-2-methyl-2-propanesulfinamide. The product $(0.94 \mathrm{~g}, 3.83 \mathrm{mmol}, 54 \%$ yield $)$ was sufficiently pure to be used in the next step.

${ }^{1}$ H NMR (600 MHz, CDCl $): \delta 8.64(\mathrm{dd}, J=2.3,0.4,1 \mathrm{H}), 8.62(\mathrm{~s}, 1 \mathrm{H}), 7.95$ (dd, $J=8.4,0.4$, $1 \mathrm{H}), 7.75$ (ddd, $J=8.3,2.4,0.4,1 \mathrm{H}), 1.24(\mathrm{~s}, 9 \mathrm{H})$.

${ }^{13}$ C NMR (150 MHz, CDCl3): $\delta$ 162.6, 150.6, 149.2, 136.6, 134.7, 123.6, 58.3, 22.8 .

HRMS (ESI-TOF): calculated for $\mathrm{C}_{10} \mathrm{H}_{14} \mathrm{ClN}_{2} \mathrm{OS}[\mathrm{M}+\mathrm{H}]^{+}: 245.0510$, found 245.0519 .

(S)-N-((S)-1-(5-chloropyridin-2-yl)-2,2-dimethylpropyl)-2-methylpropane-2-sulfinamide.

[B]

Prepared according to GP-2 on $3.40 \mathrm{mmol}$ scale. The product was obtained after column chromatography $(40 \rightarrow 60 \%$ EtOAc in hexanes) as a $4: 1$ mixture of diastereomers favoring the desired compound ( $0.52 \mathrm{~g}, 1.72 \mathrm{mmol}, 51 \%$ yield $)$.

${ }^{1}$ H NMR (500 MHz, Methanol-d4) $\delta 8.57$ (d, $\left.J=2.4 \mathrm{~Hz}, 1 \mathrm{H}\right), 7.79(\mathrm{dd}, J=8.3,2.5 \mathrm{~Hz}, 1 \mathrm{H}), 7.42$ $(\mathrm{d}, J=8.4 \mathrm{~Hz}, 1 \mathrm{H}), 5.39(\mathrm{~d}, J=8.9 \mathrm{~Hz}, 1 \mathrm{H}), 4.19(\mathrm{~d}, J=8.9 \mathrm{~Hz}, 1 \mathrm{H}), 1.32(\mathrm{~s}, 9 \mathrm{H}), 0.96(\mathrm{~s}, 9 \mathrm{H})$.

${ }^{13}$ C NMR (125 MHz, Methanol-d4) $\delta$ 159.2, 148.20, 136.9, 131.8, 126.1, 69.9, 57.6, 37.4, 27.1, 23.3 .

HRMS (ESI-TOF): calculated for $\mathrm{C}_{14} \mathrm{H}_{24} \mathrm{ClN}_{2} \mathrm{OS}[\mathrm{M}+\mathrm{H}]^{+}:$303.1292, found 303.1283.

(S)-1-(5-chloropyridin-2-yl)-2,2-dimethylpropan-1-amine dihydrochloride. [C]

Prepared according to GP-3 on $1.72 \mathrm{mmol}$ scale. The product was obtained after filtration as a brown solid (0.36 g, $1.33 \mathrm{mmol}, 78 \%$ yield).

${ }^{1}$ H NMR (500 MHz, Methanol-d4) $\delta 8.66(\mathrm{~d}, J=2.3 \mathrm{~Hz}, 1 \mathrm{H}), 7.91(\mathrm{dd}, J=8.3,2.4 \mathrm{~Hz}, 1 \mathrm{H}), 7.50$ (d, $J=8.3 \mathrm{~Hz}, 1 \mathrm{H}), 4.33$ (s, 1H), 1.04 (s, 9H).

${ }^{13}$ C NMR (125 MHz, Methanol-d4) $\delta$ 154.0, 148.9, 137.6, 133.3, 126.7, 63.9, 30.6, 26.6.

HRMS (ESI-TOF): calculated for $\mathrm{C}_{10} \mathrm{H}_{16} \mathrm{ClN}_{2}[\mathrm{M}+\mathrm{H}]^{+}:$199.0997, found 199.0987.

$N$-((S)-1-(5-chloropyridin-2-yl)-2,2-dimethylpropyl)-2,4,6-trimethylcyclohexane-1-

carboxamide 10e. Prepared according to GP-4 on $1.32 \mathrm{mmol}$ scale. The product was purified by column chromatography (15\% EtOAc in hexanes) to afford 10e $(0.32 \mathrm{~g} 0.91 \mathrm{mmol}, 69 \%$ yield).

Physical state: white crystalline solid

TLC: $\mathrm{R}_{f}=0.4\left(10 \%\right.$ EtOAc in hexanes, visualization $\left.\mathrm{UV}, \mathrm{KMnO}_{4}\right)$

m.p.: $127-129^{\circ} \mathrm{C}$

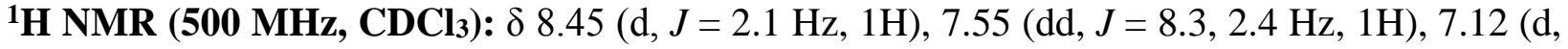
$J=8.3 \mathrm{~Hz}, 1 \mathrm{H}), 6.72(\mathrm{~d}, J=9.0 \mathrm{~Hz}, 1 \mathrm{H}), 4.88(\mathrm{~d}, J=9.3 \mathrm{~Hz}, 1 \mathrm{H}), 2.20(\mathrm{t}, J=4.5 \mathrm{~Hz}, 1 \mathrm{H}), 1.75-$ $1.57(\mathrm{~m}, 2 \mathrm{H}), 1.54(\mathrm{q}, J=11.9 \mathrm{~Hz}, 1 \mathrm{H}), 1.43-1.18(\mathrm{~m}, 4 \mathrm{H}), 1.00(\mathrm{~d}, \mathrm{~J}=6.8 \mathrm{~Hz}, 3 \mathrm{H}), 0.89$ (s, $9 \mathrm{H}), 0.88(\mathrm{~d}, J=7.0 \mathrm{~Hz}, 3 \mathrm{H}), 0.58(\mathrm{~d}, J=6.9 \mathrm{~Hz}, 3 \mathrm{H})$. 


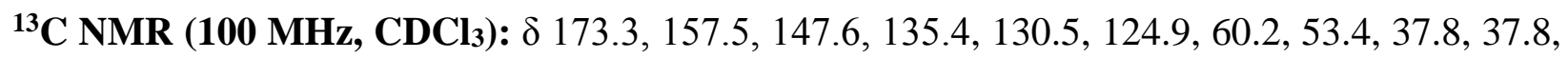
$35.7,35.3,35.1,32.7,26.9,22.5,20.4,19.9$

HRMS (m/z): calculated for $\mathrm{C}_{20} \mathrm{H}_{32} \mathrm{ClN}_{2} \mathrm{O}[\mathrm{M}+\mathrm{H}]^{+}: 351.2198$, found 351.2200.

\section{Analytical LCMS:}

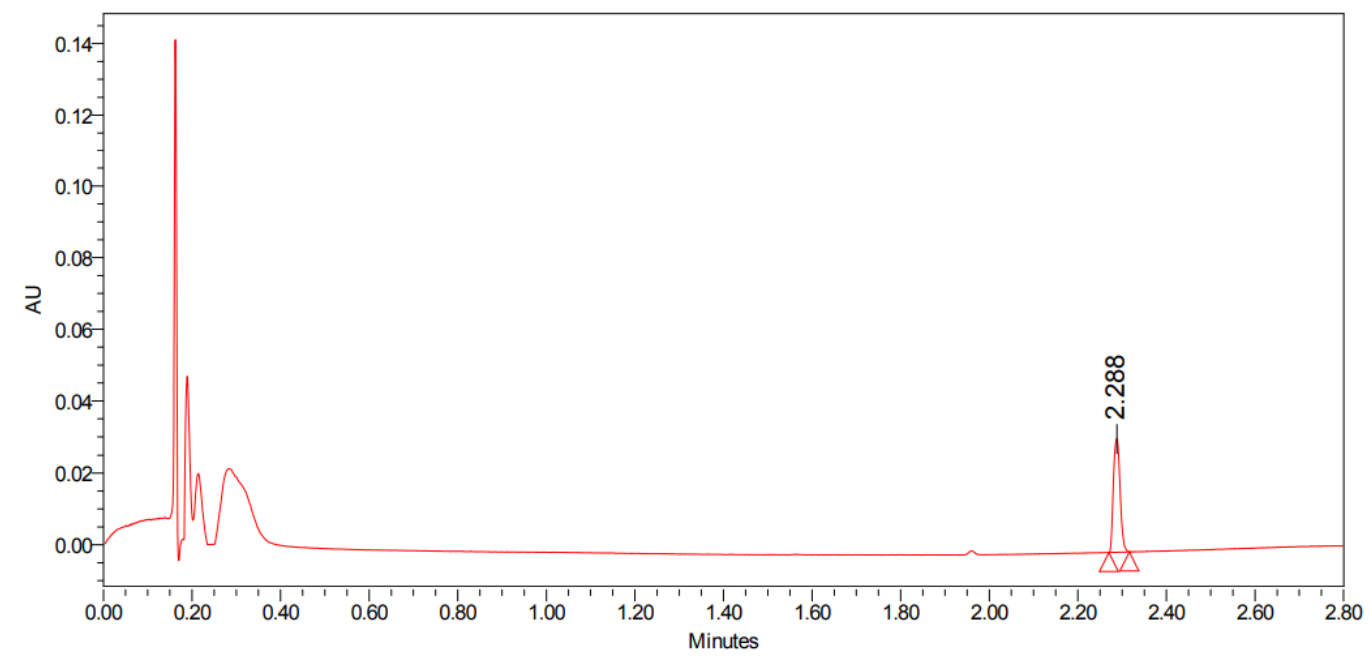

Chiral SFC: (unable to resolve enantiomers)

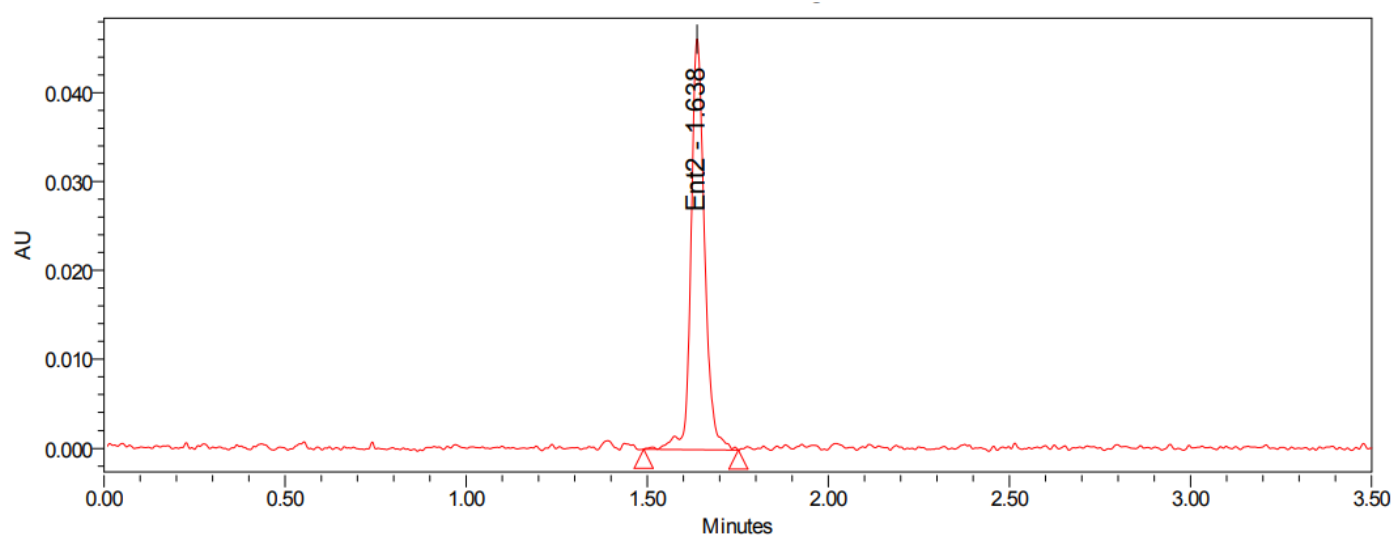




\section{Compound 10f}<smiles>CC1CC(C)C(C(=O)NC(c2ccc3ccccc3n2)C(C)(C)C)C(C)C1</smiles>

( $S, E)$-2-methyl- $N$-(quinolin-2-ylmethylene)propane-2-sulfinamide. [A]

Prepared according to GP-1 on $6.37 \mathrm{mmol}$ scale using $(S)$-(-)-2-methyl-2-propanesulfinamide. The product was obtained after purification through a plug of silica gel $\left(\mathrm{Et}_{2} \mathrm{O}\right)(1.41 \mathrm{~g}, 5.42 \mathrm{mmol}$, $85 \%$ yield).

Spectra matched the literature. ${ }^{8}$

${ }^{1} \mathbf{H}$ NMR $\left(\mathbf{5 0 0} \mathbf{M H z}, \mathbf{C D C l}_{3}\right): \delta 8.89(\mathrm{~s}, 1 \mathrm{H}), 8.26(\mathrm{~d}, J=8.5 \mathrm{~Hz}, 1 \mathrm{H}), 8.21(\mathrm{~d}, J=8.5 \mathrm{~Hz}, 1 \mathrm{H})$, $8.16(\mathrm{~d}, J=8.5 \mathrm{~Hz}, 1 \mathrm{H}), 7.88(\mathrm{~d}, J=8.2 \mathrm{~Hz}, 1 \mathrm{H}), 7.79(\mathrm{t}, J=7.7 \mathrm{~Hz}, 1 \mathrm{H}), 7.64(\mathrm{t}, J=7.5 \mathrm{~Hz}, 1 \mathrm{H})$, $1.32(\mathrm{~s}, 9 \mathrm{H})$.

(S)-N-((S)-2,2-dimethyl-1-(quinolin-2-yl)propyl)-2-methylpropane-2-sulfinamide.

Prepared according to GP-2 on $5.38 \mathrm{mmol}$ scale, the reaction was run at $-40{ }^{\circ} \mathrm{C}$. The product was obtained after column chromatography $(30 \rightarrow 60 \%$ EtOAc in hexanes) as a 5:3 mixture of diastereomers favoring the desired compound $(0.778 \mathrm{~g}, 2.45 \mathrm{mmol}, 46 \%$ yield).

${ }^{1}$ H NMR (500 MHz, CDCl $): \delta 8.08-7.99$ (combined, m, 2H), 7.79 (minor, d, $J=8.1 \mathrm{~Hz}, 1 \mathrm{H}$ ), 7.76 (major, d, $J=8.0 \mathrm{~Hz}, 1 \mathrm{H}$ ), 7.69 (minor, t, $J=7.7 \mathrm{~Hz}, 1 \mathrm{H}$ ), 7.64 (major, t, $J=7.7 \mathrm{~Hz}, 1 \mathrm{H}$ ), 7.51 (minor, t, $J=7.5 \mathrm{~Hz}, 1 \mathrm{H}$ ), 7.47 (major, t, $J=7.5 \mathrm{~Hz}, 1 \mathrm{H}$ ), 7.30 (combined, d, $J=8.4 \mathrm{~Hz}, 1 \mathrm{H}$ ), 5.78 (major, d, $J=7.3 \mathrm{~Hz}, 1 \mathrm{H}$ ), 4.90 (minor, d, $J=6.6 \mathrm{~Hz}, 1 \mathrm{H}$ ), 4.35 (minor, d, $J=6.7 \mathrm{~Hz}, 1 \mathrm{H}$ ), 4.24 (major, d, $J=7.4 \mathrm{~Hz}, 1 \mathrm{H}$ ), 1.32 (major, s, 9H), 1.08 (minor, s, 9H), 1.02 (minor, s, 9H), 0.95 (major, s, 9H).

${ }^{13}$ C NMR (125 MHz, CDCl3): $\delta 160.0$ (major), 159.9 (minor), 147.3 (minor), 147.1 (major), 135.4 (combined), 129.5 (minor), 129.4 (major), 129.4 (minor), 129.3 (major), 127.6 (minor), 127.5 (major), 127.4 (major), 127.2 (minor), 126.4 (minor), 126.2 (major), 122.3 (major), 121.8 (minor), 69.4 (minor), 68.4 (major), 56.3 (combined), 37.0 (major), 36.4 (minor), 27.0 (minor), 26.8 (major), 23.2 (major), 22.4 (minor).

HRMS (ESI-TOF): calculated for $\mathrm{C}_{24} \mathrm{H}_{35} \mathrm{~N}_{2} \mathrm{O}[\mathrm{M}+\mathrm{H}]^{+}: 319.1839$, found 319.1835 . 
(S)-2,2-dimethyl-1-(quinolin-2-yl)propan-1-amine dihydrochloride. [C]

Prepared according to GP-3 on $2.45 \mathrm{mmol}$ scale. The product was obtained after filtration as a yellow solid (0.662 $\mathrm{g}, 2.31 \mathrm{mmol}, 94 \%$ yield).

${ }^{1}$ H NMR (500 MHz, DMSO-d6): $\delta 8.89$ (s, 3H), $8.64(\mathrm{~d}, J=7.1 \mathrm{~Hz}, 1 \mathrm{H}), 8.20(\mathrm{~d}, J=8.3 \mathrm{~Hz}, 1 \mathrm{H})$, $8.12(\mathrm{~d}, J=8.1 \mathrm{~Hz}, 1 \mathrm{H}), 7.90(\mathrm{t}, J=7.6 \mathrm{~Hz}, 1 \mathrm{H}), 7.84(\mathrm{~d}, J=8.0 \mathrm{~Hz}, 1 \mathrm{H}), 7.72(\mathrm{t}, J=7.5 \mathrm{~Hz}, 1 \mathrm{H})$, $4.57(\mathrm{~s}, 1 \mathrm{H}), 1.02(\mathrm{~s}, 9 \mathrm{H})$.

${ }^{13}$ C NMR (150 MHz, DMSO-d6): 155.5, 144.5, 138.3, 131.2, 128.3, 127.7, 127.4, 126.9, 122.1, $62.1,34.7,26.4$.

HRMS (ESI-TOF): calculated for $\mathrm{C}_{14} \mathrm{H}_{19} \mathrm{~N}_{2}[\mathrm{M}+\mathrm{H}]^{+}:$215.1543, found 215.1545.

$N$-(S)-2,2-dimethyl-1-(quinolin-2-yl)propyl)-2,4,6-trimethylcyclohexane-1-carboxamide $10 f$. Prepared according to GP-4 on $1.30 \mathrm{mmol}$ scale. Purified by column chromatography (10\% EtOAc in hexanes) to afford $(0.31 \mathrm{~g}, 0.85 \mathrm{mmol}, 60 \%$ yield $)$ of the title compound $\mathbf{1 0 f}$.

Physical state: yellow solid

TLC: $\mathrm{R}_{f}=0.5\left(20 \%\right.$ EtOAc in hexanes, visualization $\left.\mathrm{UV}, \mathrm{KMnO}_{4}\right)$

m.p.: $138-140{ }^{\circ} \mathrm{C}$

${ }^{1}$ H NMR (500 MHz, CDCl3): $\delta 8.03(\mathrm{dd}, J=8.3,4.0 \mathrm{~Hz}, 2 \mathrm{H}), 7.80(\mathrm{~d}, J=8.0 \mathrm{~Hz}, 1 \mathrm{H}), 7.68(\mathrm{t}, J$ $=7.7 \mathrm{~Hz}, 1 \mathrm{H}), 7.51(\mathrm{t}, J=7.4 \mathrm{~Hz}, 1 \mathrm{H}), 7.30(\mathrm{~d}, J=8.4 \mathrm{~Hz}, 1 \mathrm{H}), 7.17(\mathrm{~d}, J=8.7 \mathrm{~Hz}, 1 \mathrm{H}), 5.09(\mathrm{~d}$, $J=9.1 \mathrm{~Hz}, 1 \mathrm{H}), 2.33(\mathrm{t}, J=4.6 \mathrm{~Hz}, 1 \mathrm{H}), 1.74-1.59(\mathrm{~m}, 3 \mathrm{H}), 1.42-1.28(\mathrm{~m}, 3 \mathrm{H}), 1.23(\mathrm{~d}, J=$ $12.5 \mathrm{~Hz}, 1 \mathrm{H}), 1.06(\mathrm{~d}, J=6.7 \mathrm{~Hz}, 3 \mathrm{H}), 0.98(\mathrm{~s}, 9 \mathrm{H}), 0.91(\mathrm{~d}, J=6.2 \mathrm{~Hz}, 3 \mathrm{H}), 0.57$ (d, $J=6.9 \mathrm{~Hz}$, $3 \mathrm{H})$.

${ }^{13}$ C NMR (125 MHz, CDCl3): $\delta$ 173.3, 159.8, 147.4, 135.3, 129.4, 129.3, 127.7, 127.3, 126.2, 122.7, 61.0, 53.4, 37.9, 37.8, 36.3, 35.3, 35.1, 32.7, 27.1, 22.5, 20.3, 20.0.

HRMS (ESI-TOF): calculated for $\mathrm{C}_{24} \mathrm{H}_{35} \mathrm{~N}_{2} \mathrm{O}[\mathrm{M}+\mathrm{H}]^{+}:$367.2744, found 367.2756.

\section{Analytical LCMS:}

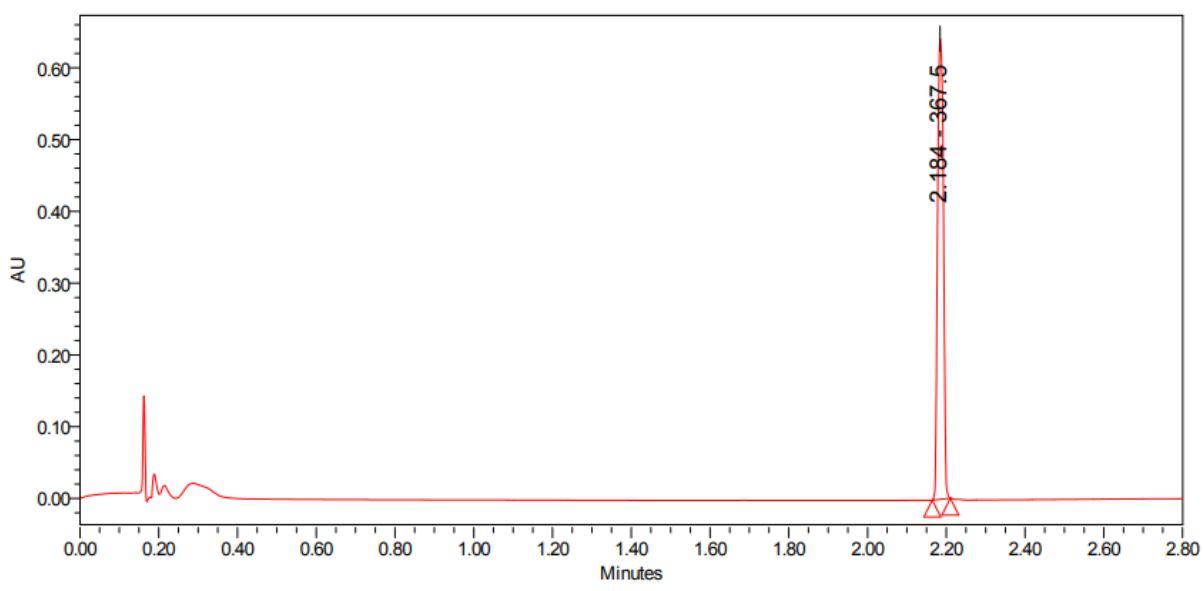


Chiral SFC: (Ent1 33.8\%, Ent2 66.2\%)

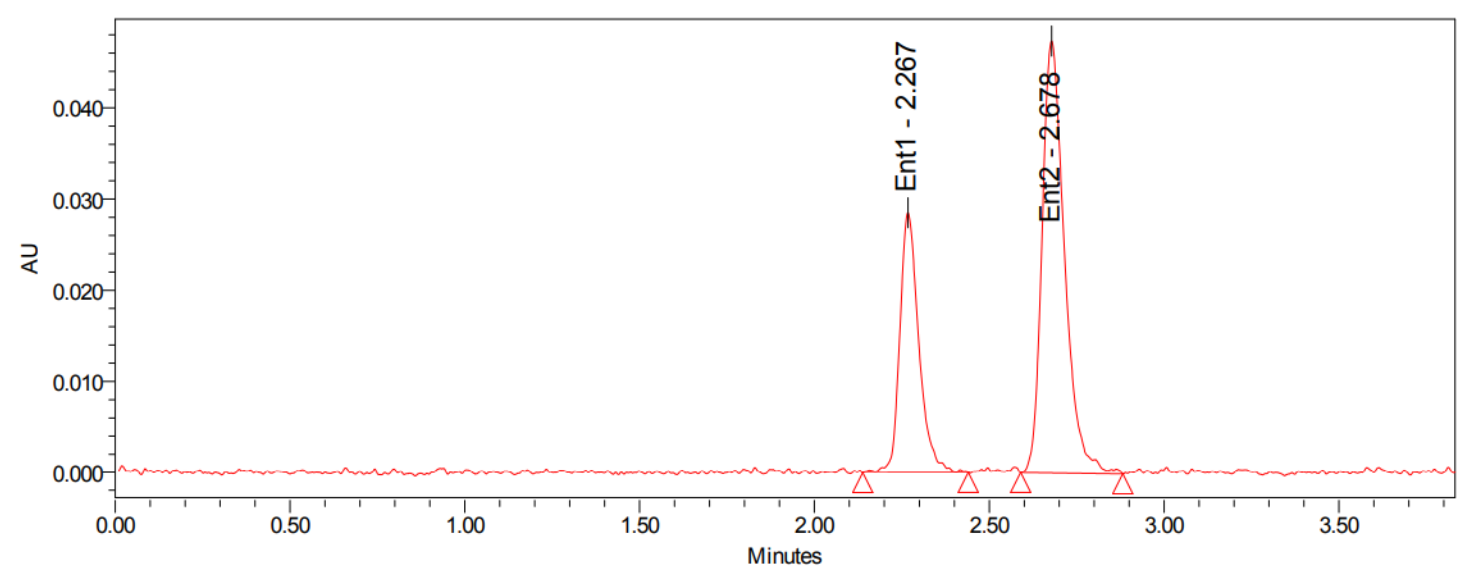

\section{C-H Activation DG Comparison}

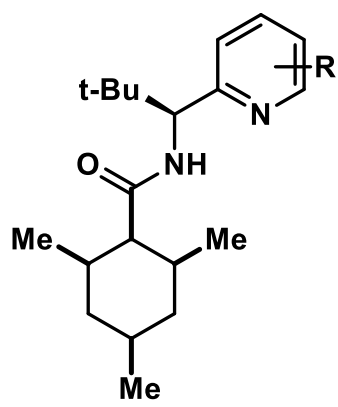

$\mathrm{Pd}(\mathrm{OAc})_{2}(15 \mathrm{~mol} \%)$, LiOAc (1 equiv.), $\mathrm{Ac}_{2} \mathrm{O}$ (2 equiv.) $\mathrm{NaIO}_{4}$ (4 equiv.)

$\mathrm{PhMe} / \mathrm{MeOH}$ (2:1), $90{ }^{\circ} \mathrm{C}, 22 \mathrm{hrs}$.

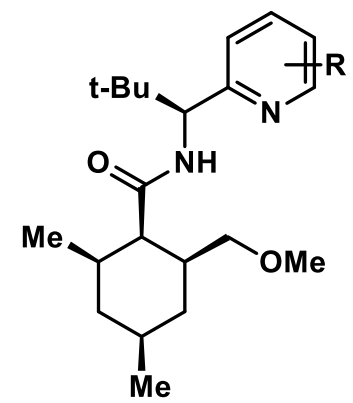

\begin{tabular}{ll}
$(\mathrm{R}=)$ & yield \\
\hline $10 \mathrm{a}(\mathrm{H})$ & $44 \%$
\end{tabular} $10 \mathrm{~b}(6-\mathrm{OMe}) \quad$ n.r. 10 c (4-Me) $31 \%$ SI-3 (4-Cl) $\quad 56 \%$ 10 e $(5-\mathrm{Cl}) \quad 53 \%$ $10 f$ (Quin) 23\%

General Procedure 5 (GP-5): An oven dried small culture tube equipped with stir bar was charged with $\mathrm{C}-\mathrm{H}$ activation substrate $\left(0.1 \mathrm{mmol}, 1.00\right.$ equiv.), $\mathrm{Pd}(\mathrm{OAc})_{2}(0.015 \mathrm{mmol}, 0.15$ equiv.), $\mathrm{LiOAc}\left(0.1 \mathrm{mmol}, 1.00\right.$ equiv.) and $\mathrm{NaIO}_{4}(0.4 \mathrm{mmol}, 4.00$ equiv.). The tube was evacuated and backfilled with argon (repeated twice) before sealing with a septum screw cap. A 2:1 mixture of anhydrous $\mathrm{PhMe} / \mathrm{MeOH}(2 \mathrm{~mL}, 0.05 \mathrm{M})$ was added, followed by acetic anhydride ( $0.2 \mathrm{mmol}, 2.00$ equiv.). The cap was quickly replaced with a PTFE lined solid screw cap and the mixture was transferred to an oil bath pre-heated to $90{ }^{\circ} \mathrm{C}$. The mixture was allowed to stir at this temperature for 22 hours, before cooling and diluting with $\mathrm{Et}_{2} \mathrm{O}$. The suspension was filtered over Celite $^{\circledR}$ and concentrated under reduced pressure. Purification by preparative thin-layer chromatography provided the de-symmetrized product. In all cases product d.r. exceeded 10:1 by crude NMR. 


\section{Compound SI-4}<smiles>COCC1CC([Si])CC(C)C1C(=O)NC(c1ccccn1)C(C)(C)C</smiles>

Prepared according to GP-5 on $0.1 \mathrm{mmol}$ scale. The product SI-4 (15.2 mg, $0.044 \mathrm{mmol}$, 44\% yield) was obtained after preparative thin layer chromatography (25\% EtOAc in hexanes).

${ }^{1}$ H NMR (600 MHz, CDCl $): \delta 8.51(\mathrm{~d}, J=4.0 \mathrm{~Hz}, 1 \mathrm{H}), 7.57(\mathrm{td}, J=7.6,1.8 \mathrm{~Hz}, 1 \mathrm{H}), 7.18-$ $7.10(\mathrm{~m}, 2 \mathrm{H}), 6.96(\mathrm{~d}, J=9.0 \mathrm{~Hz}, 1 \mathrm{H}), 4.87(\mathrm{~d}, J=9.2 \mathrm{~Hz}, 1 \mathrm{H}), 3.38-3.35(\mathrm{~m}, 4 \mathrm{H}), 3.27(\mathrm{dd}, J$ $=9.2,5.5 \mathrm{~Hz}, 1 \mathrm{H}), 2.50(\mathrm{t}, J=4.6 \mathrm{~Hz}, 1 \mathrm{H}), 1.96-1.87(\mathrm{~m}, 1 \mathrm{H}), 1.66-1.57(\mathrm{~m}, 1 \mathrm{H}), 1.53(\mathrm{q}, J$ $=12.3 \mathrm{~Hz}, 1 \mathrm{H}), 1.47-1.34(\mathrm{~m}, 2 \mathrm{H}), 1.34-1.29(\mathrm{~m}, 1 \mathrm{H}), 1.28-1.22(\mathrm{~m}, 1 \mathrm{H}), 0.93(\mathrm{~s}, 9 \mathrm{H}), 0.90$ $(\mathrm{d}, J=6.0 \mathrm{~Hz}, 3 \mathrm{H}), 0.58(\mathrm{~d}, J=6.9 \mathrm{~Hz}, 3 \mathrm{H})$.

${ }^{13}$ C NMR (150 MHz, CDCl3): $\delta$ 172.9, 159.2, 148.7, 135.6, 124.2, 122.1, 76.2, 60.9, 58.9, 48.1, $40.9,38.2,35.7,34.9,32.5,32.3,26.9,22.5,19.7$.

HRMS (ESI-TOF): calculated for $\mathrm{C}_{21} \mathrm{H}_{35} \mathrm{~N}_{2} \mathrm{O}_{2}{ }^{+}[\mathrm{M}+\mathrm{H}]^{+}:$347.2693, found 347.2695.

\section{Compound SI-5}<smiles>COCC1CC([Si])CC(C)C1C(=O)NC(c1cc(C)ccn1)C(C)(C)C</smiles>

Prepared according to GP-5 on $0.1 \mathrm{mmol}$ scale. The product SI-5 (11.2 mg, $0.031 \mathrm{mmol}, 31 \%$ yield) was obtained after preparative thin layer chromatography (25\% EtOAc in hexanes).

${ }^{1}$ H NMR (600 MHz, CDCl3): $\delta 8.35(\mathrm{~d}, J=4.9 \mathrm{~Hz}, 1 \mathrm{H}), 6.98-6.93(\mathrm{~m}, 3 \mathrm{H}), 4.81(\mathrm{~d}, J=9.3 \mathrm{~Hz}$, $1 \mathrm{H}), 3.38-3.35(\mathrm{~m}, 4 \mathrm{H}), 3.27(\mathrm{~d}, J=9.2,5.6 \mathrm{~Hz}, 1 \mathrm{H}), 2.50(\mathrm{t}, J=4.6 \mathrm{~Hz}, 1 \mathrm{H}), 2.31(\mathrm{~s}, 3 \mathrm{H}), 1.94$ $-1.89(\mathrm{~m}, 1 \mathrm{H}), 1.64-1.60(\mathrm{~m}, 1 \mathrm{H}), 1.54(\mathrm{q}, J=12.2 \mathrm{~Hz}, 1 \mathrm{H}), 1.43-1.37(\mathrm{~m}, 2 \mathrm{H}), 1.33-1.31$ $(\mathrm{m}, 1 \mathrm{H}), 1.28-1.25(\mathrm{~m}, 1 \mathrm{H}), 0.95-0.88(\mathrm{~m}, 12 \mathrm{H}), 0.59(\mathrm{~d}, J=6.9 \mathrm{~Hz}, 3 \mathrm{H})$. 
${ }^{13}$ C NMR (150 MHz, $\left.\mathbf{C D C l}_{3}\right): \delta$ 172.9, 158.9, 148.4, 146.7, 125.1, 123.1, 76.2, 60.8, 58.9, 48.1, $41.0,38.2,35.6,34.9,32.5,32.3,27.0,22.5,21.2,19.7$.

HRMS (ESI-TOF): calculated for $\mathrm{C}_{22} \mathrm{H}_{37} \mathrm{~N}_{2} \mathrm{O}_{2}{ }^{+}[\mathrm{M}+\mathrm{H}]^{+}: 361.2850$, found 361.2848.

\section{Compound SI-6}<smiles>COCC1C[C@H]([N+](=O)[O-])C[C@H](C)C1C(=O)NC(c1ccccc1)c1cc(Cl)ccn1</smiles>

Prepared according to GP-5 on $0.1 \mathrm{mmol}$ scale. The product SI-6 $(21.3 \mathrm{mg}, 0.056 \mathrm{mmol}, 56 \%$ yield) was obtained after preparative thin layer chromatography (20\% EtOAc in hexanes).

For spectral data see compound 11.

\section{Compound SI-7}<smiles>B#[N+]C1CC(COC)C(C(=O)NC(c2ccc(Cl)cn2)C(C)(C)C)[C@@H](C)C1</smiles>

Prepared according to GP-5 on $0.1 \mathrm{mmol}$ scale. The product SI-7 $(20.2 \mathrm{mg}, 0.053 \mathrm{mmol}, 53 \%$ yield) was obtained after preparative thin layer chromatography (20\% EtOAc in hexanes).

${ }^{1} \mathbf{H}$ NMR $\left(600 \mathrm{MHz}, \mathbf{C D C l}_{3}\right): \delta 8.5(\mathrm{~d}, J=2.4 \mathrm{~Hz}, 1 \mathrm{H}), 7.56(\mathrm{dd}, J=8.3,2.5 \mathrm{~Hz}, 1 \mathrm{H}), 7.12(\mathrm{~d}, J$ $=8.3 \mathrm{~Hz}, 1 \mathrm{H}), 6.81(\mathrm{~d}, J=9.2 \mathrm{~Hz}, 1 \mathrm{H}), 4.87(\mathrm{~d}, J=9.2 \mathrm{~Hz}, 1 \mathrm{H}), 3.36-3.35(\mathrm{~m}, 4 \mathrm{H}), 3.26(\mathrm{dd}, J$ $=9.2,5.4 \mathrm{~Hz}, 1 \mathrm{H}), 2.49(\mathrm{t}, J=4.6 \mathrm{~Hz}, 1 \mathrm{H}), 1.95-1.89(\mathrm{~m}, 1 \mathrm{H}), 1.66-1.58(\mathrm{~m}, 1 \mathrm{H}), 1.54-1.45$ $(\mathrm{m}, 1 \mathrm{H}), 1.46-1.34(\mathrm{~m}, 2 \mathrm{H}), 1.33-1.23(\mathrm{~m}, 2 \mathrm{H}), 0.92(\mathrm{~s}, 9 \mathrm{H}), 0.90(\mathrm{~d}, J=5.9 \mathrm{~Hz}, 3 \mathrm{H}), 0.60(\mathrm{~d}$, $J=6.9 \mathrm{~Hz}, 3 \mathrm{H})$. 
${ }^{13}$ C NMR (150 MHz, CDCl $): ~ \delta 172.9,157.5,147.7,135.4,130.5,124.8,76.1,60.4,58.9,48.1$, $40.9,38.2,35.6,34.9,32.4,32.3,26.8,22.5,19.8$.

HRMS (ESI-TOF): calculated for $\mathrm{C}_{21} \mathrm{H}_{34} \mathrm{ClN}_{2} \mathrm{O}_{2}[\mathrm{M}+\mathrm{H}]^{+}: 381.2303$, found 381.2310.

\section{Compound SI-8}<smiles>COCC1C[C@H](N)C[C@@H](C)[C@H]1C(=O)NC(c1ccc2ccccc2n1)C(C)(C)C</smiles>

Prepared according to GP-5 on $0.1 \mathrm{mmol}$ scale. The product SI-8 $(9.1 \mathrm{mg}, 0.023 \mathrm{mmol}, 23 \%$ yield) was obtained after preparative thin layer chromatography (15\% EtOAc in hexanes).

${ }^{1} \mathbf{H}$ NMR $\left(600 \mathrm{MHz}, \mathbf{C D C l}_{3}\right): \delta 8.03(\mathrm{t}, J=8.1 \mathrm{~Hz}, 2 \mathrm{H}), 7.79(\mathrm{~d}, J=8.0 \mathrm{~Hz}, 1 \mathrm{H}), 7.72-7.66(\mathrm{~m}$, $1 \mathrm{H}), 7.53-7.48(\mathrm{~m}, 1 \mathrm{H}), 7.31-7.27(\mathrm{~m}, 2 \mathrm{H}), 5.06(\mathrm{~d}, \mathrm{~J}=9.0 \mathrm{~Hz}, 1 \mathrm{H}), 3.44-3.41(\mathrm{~m}, 4 \mathrm{H}), 3.32$ $(\mathrm{dd}, J=9.1,5.4 \mathrm{~Hz}, 1 \mathrm{H}), 2.60(\mathrm{t}, J=4.6 \mathrm{~Hz}, 1 \mathrm{H}), 1.99-1.89(\mathrm{~m}, 1 \mathrm{H}), 1.67-1.53(\mathrm{~m}, 2 \mathrm{H}), 1.48$ $-1.30(\mathrm{~m}, 3 \mathrm{H}), 1.24(\mathrm{~d}, \mathrm{~J}=12.3 \mathrm{~Hz}, 1 \mathrm{H}), 0.98(\mathrm{~s}, 9 \mathrm{H}), 0.91(\mathrm{~d}, J=6.2 \mathrm{~Hz}, 3 \mathrm{H}), 0.56(\mathrm{~d}, J=6.9$ $\mathrm{Hz}, 3 \mathrm{H})$.

${ }^{13}$ C NMR (150 MHz, CDCl3): $\delta 172.9,159.7,147.5,135.3,129.4,129.4,127.7,127.3,126.2$, 122.6, 76.3, 61.2, 59.0, 48.2, 41.1, 38.2, 36.4, 34.9, 32.5, 32.4, 27.1, 22.5, 19.9 .

HRMS (ESI-TOF): calculated for $\mathrm{C}_{25} \mathrm{H}_{37} \mathrm{~N}_{2} \mathrm{O}_{2}[\mathrm{M}+\mathrm{H}]^{+}:$397.2850, found 397.2847.

\section{Synthesis of (-)-Maximiscin}

\section{Compound 7}

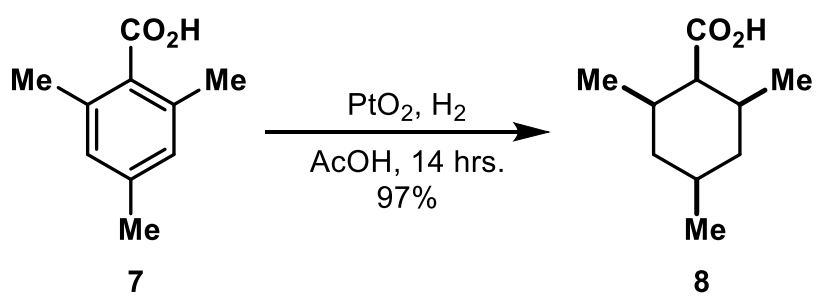

Prepared according to a modified literature procedure. ${ }^{9}$ A $100 \mathrm{~mL}$ round-bottomed flask was charged with a wide stir bar, 7 (7.00 g, 42.7 mmol, 1.00 equiv.) and Platinum(IV) oxide (0.48 g, 
$2.13 \mathrm{mmol}, 0.05$ equiv.) then dissolved in $34 \mathrm{~mL}$ glacial acetic acid. The flask was placed in a Parr high pressure hydrogenation apparatus (Note 1) and sealed. The vessel was slowly pressurized to 400 psi with hydrogen gas, then allowed to vent; this process was repeated three times. After repressurization to 450 psi with hydrogen, the vessel was sealed and placed on a stir plate at 1400 rpm behind a blast shield. After 14 hours, the vessel was slowly depressurized and opened to reveal a clear reaction mixture with black precipitate. The mixture was filtered over Celite ${ }^{\circledR}$, and the resulting acetic acid solution was blown down using compressed air over several hours to afford a white solid. This solid was re-suspended in toluene and subjected to rotary evaporation to afford pure 8 ( $7.07 \mathrm{~g}, 41.6 \mathrm{mmol}, 97 \%$ yield $)$.

Note 1 - The flask was supported by a rubber filter adapter to keep it upright inside the vessel.

Spectral data consistent with the literature. ${ }^{9}$

Physical state: white solid

TLC: $\mathrm{R}_{f}=0.3$ (10\% EtOAc in hexanes; visualization by bromocresol green)

m.p.: $84-86^{\circ} \mathrm{C}$

${ }^{1} \mathbf{H}$ NMR $\left(600 \mathrm{MHz}, \mathbf{C D C l}_{3}\right): \delta 2.56(\mathrm{t}, J=4.9 \mathrm{~Hz}, 1 \mathrm{H}), 1.73(\mathrm{ddt}, J=8.7,6.9,4.7 \mathrm{~Hz}, 2 \mathrm{H}), 1.40$ $-1.47(\mathrm{~m}, 1 \mathrm{H}), 1.38(\mathrm{dt}, J=13.1,3.6 \mathrm{~Hz}, 2 \mathrm{H}), 1.30(\mathrm{q}, J=12.3 \mathrm{~Hz}, 2 \mathrm{H}), 0.99(\mathrm{~d}, J=7.0 \mathrm{~Hz}, 6 \mathrm{H})$, $0.92(\mathrm{~d}, J=6.4 \mathrm{~Hz}, 3 \mathrm{H})$.

${ }^{13} \mathbf{C}$ NMR (150 MHz, $\left.\mathbf{C D C l}_{3}\right): \delta 179.9,51.2,37.5,34.5,32.5,22.4,20.0$.

HRMS (m/z): calculated for $\mathrm{C}_{10} \mathrm{H}_{17} \mathrm{O}_{2}[\mathrm{M}-\mathrm{H}]^{-}:$: 69.1234 , found 169.1224 .

\section{Compound SI-9}

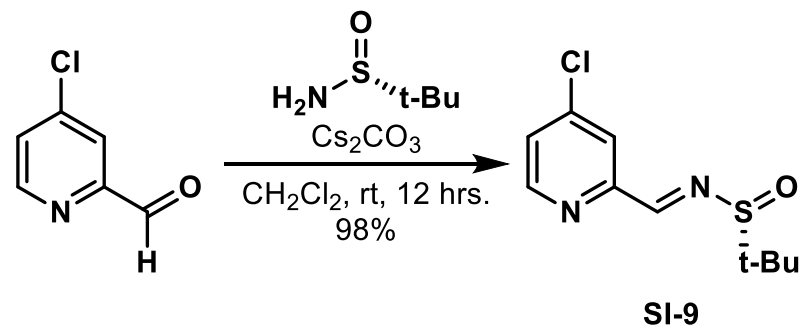

Prepared according to a modified literature procedure. ${ }^{10}$ A $500 \mathrm{~mL}$ round-bottomed flask was charged with a stir bar, 4-chloropyridine-2-carbaldehyde (11.9 g, $84.0 \mathrm{mmol}, 1.00$ equiv.), $(R)$ (+)-2-methyl-2-propanesulfinamide (10.2 g, $84 \mathrm{mmol}, 1.00$ equiv.), anhydrous cesium carbonate (32.8 g, $101 \mathrm{mmol}, 1.20$ equiv.) and $280 \mathrm{~mL}(0.3 \mathrm{M}) \mathrm{CH}_{2} \mathrm{Cl}_{2}$. The reaction was stirred vigorously overnight, then diluted with $\mathrm{CH}_{2} \mathrm{Cl}_{2}(c a .200 \mathrm{~mL})$ and filtered over Celite ${ }^{\circledR}$ into a separatory funnel. The organic layer was washed with water $(250 \mathrm{~mL})$, dried with sodium sulfate, filtered and concentrated under reduced pressure. The resulting oil was purified by silica gel chromatography (30\% $\mathrm{Et}_{2} \mathrm{O}$ in hexanes) to afford the title compound SI-9 (20.1 g, $82 \mathrm{mmol}, 98 \%$ yield). 
Physical state: pale yellow oil

TLC: $\mathrm{R}_{f}=0.4\left(30 \%\right.$ EtOAc in hexanes; visualization by $\left.\mathrm{UV}, \mathrm{KMnO}_{4}\right)$

$[\alpha]_{D}^{20.0}=-74.6\left(c=1.0, \mathrm{CHCl}_{3}\right)$

${ }^{1}$ H NMR (600 MHz, CDCl 3$): ~ \delta 8.64(\mathrm{~s}, 1 \mathrm{H}), 8.61(\mathrm{~d}, J=5.3 \mathrm{~Hz}, 1 \mathrm{H}), 7.99(\mathrm{~d}, J=1.7 \mathrm{~Hz}, 1 \mathrm{H})$, $7.38(\mathrm{dd}, J=5.3,2.0 \mathrm{~Hz}, 1 \mathrm{H}), 1.26(\mathrm{~s}, 9 \mathrm{H})$.

${ }^{13}$ C NMR (150 MHz, CDCl3): $\delta$ 162.9, 153.9, 151.0, 145.1, 126.1, 123.1, 58.4, 22.8.

HRMS (m/z): calculated for $\mathrm{C}_{10} \mathrm{H}_{14} \mathrm{ClN}_{2} \mathrm{OS}[\mathrm{M}+\mathrm{H}]^{+}:$245.0510, found 245.0516.

\section{Compound SI-10}

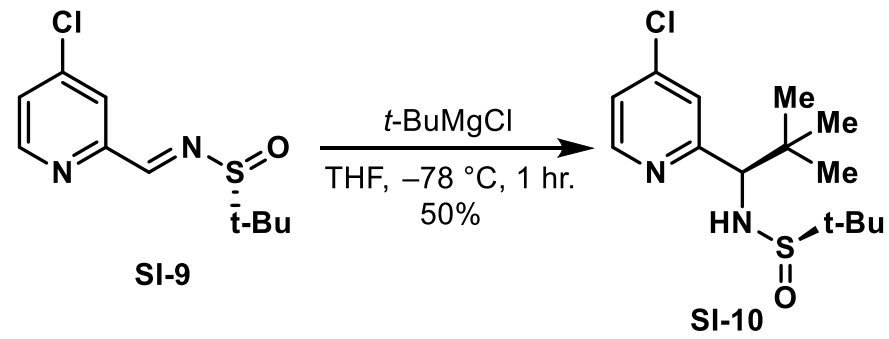

A $500 \mathrm{~mL}$ flame dried round-bottomed flask was charged with stir bar, SI-9 $(20.1 \mathrm{~g}, 82.0 \mathrm{mmol}$, 1.00 equiv. ) and anhydrous THF $(273 \mathrm{ml}, 0.3 \mathrm{M})$, then cooled to $-78{ }^{\circ} \mathrm{C}$. A $1 \mathrm{M}$ solution of tertbutyl magnesium chloride in THF (98.4 mL, $98.4 \mathrm{mmol}, 1.20$ equiv.) was slowly added (Note 1). The reaction was allowed to stir at this temperature for one hour, until complete by TLC (40\% EtOAc in hexanes). Saturated aqueous ammonium chloride $(100 \mathrm{~mL})$ was added, followed by 100 $\mathrm{mL}$ water and $150 \mathrm{~mL}$ EtOAc; the mixture was allowed to warm to ambient temperature with stirring. The aqueous layer was extracted with EtOAc $(2 \mathrm{X} 100 \mathrm{~mL})$ and the combined organics were washed with brine $(200 \mathrm{~mL})$, dried with sodium sulfate, filtered and concentrated in-vacuo to afford a brown oil. Purification by column chromatography $(20 \rightarrow 40 \%$ EtOAc in hexanes) (Note 2) provided pure SI-10 as a brown oil (12.5 g, $41.3 \mathrm{mmol}, 50 \%$ yield). Crystalization of this material from hexanes at $-20{ }^{\circ} \mathrm{C}$ provided material suitable for $\mathrm{x}$-ray analysis and removed the brown color. (Note 3)

Note 1 - The Grignard reagent was added via cannula transfer from a $100 \mathrm{~mL}$ commercial bottle (Sigma). The addition took approximately 10 mins.

Note 2 - Compound is streaky on silica gel and was collected over numerous fractions. Minor diastereomer and major impurities elute just prior to the product.

Note 3 - The brown color does not affect NMR purity or reactivity in subsequent steps. Recrystallization can be used to remove it (vide supra).

Physical state: tan crystals 
TLC: $\mathrm{R}_{f}=0.25$ (30\% EtOAc in hexanes; visualization by $\mathrm{UV}, \mathrm{KMnO}_{4}$ )

m.p.: $96-97{ }^{\circ} \mathrm{C}$

$[\alpha]_{D}^{20.0}=-185.0\left(c=1.0, \mathrm{CHCl}_{3}\right)$

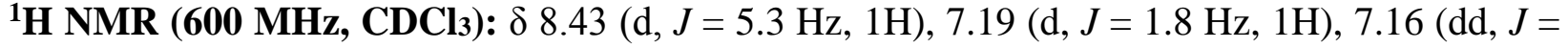
$5.3,1.9 \mathrm{~Hz}, 1 \mathrm{H}), 5.16(\mathrm{~d}, J=8.2 \mathrm{~Hz}, 1 \mathrm{H}), 3.98(\mathrm{~d}, J=8.2 \mathrm{~Hz}, 1 \mathrm{H}), 1.28(\mathrm{~s}, 9 \mathrm{H}), 0.91(\mathrm{~s}, 9 \mathrm{H})$.

${ }^{13}$ C NMR (150 MHz, CDCl3): $\delta$ 161.4, 149.6, 143.8, 124.2, 122.7, 68.8, 56.4, 36.7, 26.8, 23.1.

HRMS (m/z): calculated for $\mathrm{C}_{14} \mathrm{H}_{24} \mathrm{ClN}_{2} \mathrm{OS}[\mathrm{M}+\mathrm{H}]^{+}: 303.1292$, found 303.1299.

\section{X-ray Structure SI-10:}

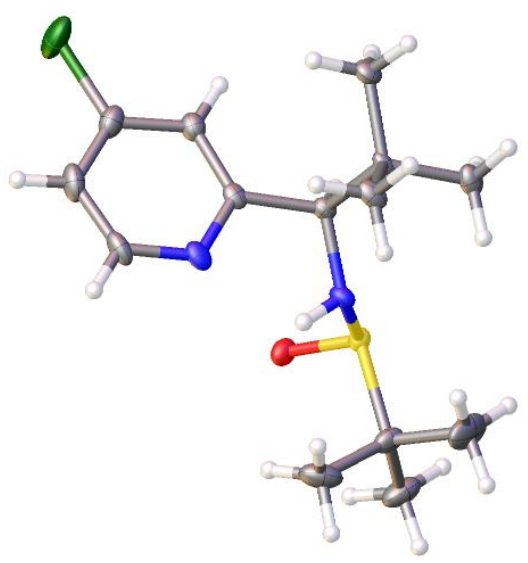

\section{Experimental Summary}

The single crystal X-ray diffraction studies were carried out on a Bruker X8-ApexII diffractometer equipped with $\mathrm{Mo} \mathrm{K}_{\alpha}$ radiation $(\lambda=0.7107 \AA)$.

Crystals of the subject compound were used as received (grown from $\mathrm{Et}_{2} \mathrm{O} / \mathrm{Hexanes}$ ). A $0.34 \times 0.12$ x $0.12 \mathrm{~mm}$ piece of a colorless crystal was mounted on a Cryoloop with Paratone oil. Data were collected in a nitrogen gas stream at 100(2) $\mathrm{K}$ using $\varpi$ scans. Crystal-to-detector distance was $40 \mathrm{~mm}$ and exposure time was 10 or 12 seconds depending on the $2 \theta$ range per frame using a scan width of $0.7^{\circ}$. Data collection was $99.9 \%$ complete to $25.242^{\circ}$ in $\theta$. A total of 35841 reflections were collected covering the indices, $9<=\mathrm{h}<=10,-16<=\mathrm{k}<=19,-29=1<=29.6532$ reflections were found to be symmetry independent, with a $\mathrm{R}_{\text {int }}$ of 0.0543. Indexing and unit cell refinement indicated a Primitive, Orthorhombic lattice. The space group was found to be $\boldsymbol{P} \mathbf{2}_{1} \mathbf{2}_{1} \mathbf{2}_{1}$. The data were integrated using the Bruker SAINT Software program and scaled using the SADABS software program. Solution by direct methods (SHELXT) produced a complete phasing model consistent with the proposed structure.

All nonhydrogen atoms were refined anisotropically by full-matrix least-squares (SHELXL-2014). All carbon bonded hydrogen atoms were placed using a riding model. Their positions were constrained 
relative to their parent atom using the appropriate HFIX command in SHELXL-2014. Crystallographic data are summarized in Table 1.

Notes: Great data! Absolute stereochemistry was conclusively assigned (Flack $=0.01(3))$.

Table 1. Crystal data and structure refinement for Baran743.

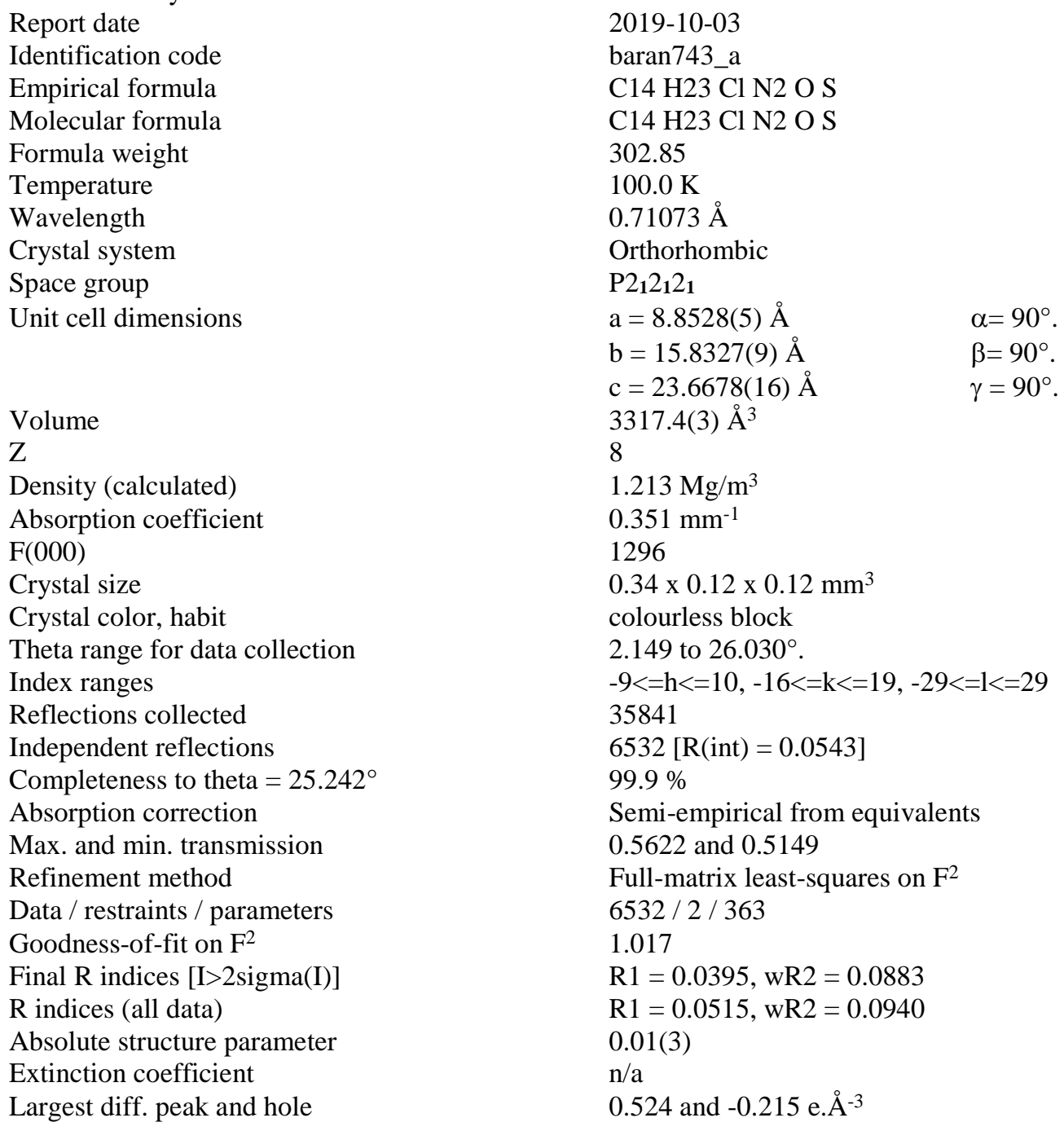

Table 2. Atomic coordinates $\left(\mathrm{x} 10^{4}\right)$ and equivalent isotropic displacement parameters $\left(\AA^{2} \times 10^{3}\right)$ for Baran743. $\mathrm{U}(\mathrm{eq})$ is defined as one third of the trace of the orthogonalized $\mathrm{U}^{\mathrm{ij}}$ tensor.

\begin{tabular}{lrrrc}
\hline & $\mathrm{x}$ & $\mathrm{y}$ & $\mathrm{z}$ & $\mathrm{U}(\mathrm{eq})$ \\
\hline $\mathrm{Cl}(1 \mathrm{~A})$ & $10004(1)$ & $5926(1)$ & $1390(1)$ & $43(1)$ \\
$\mathrm{S}(1 \mathrm{~A})$ & $7831(1)$ & $3671(1)$ & $3876(1)$ & $15(1)$ \\
$\mathrm{O}(1 \mathrm{~A})$ & $6537(2)$ & $3980(2)$ & $3531(1)$ & $21(1)$ \\
$\mathrm{N}(1 \mathrm{~A})$ & $9475(3)$ & $5760(2)$ & $3278(1)$ & $20(1)$ \\
$\mathrm{N}(2 \mathrm{~A})$ & $9310(3)$ & $4314(2)$ & $3846(1)$ & $16(1)$ \\
$\mathrm{C}(1 \mathrm{~A})$ & $9186(4)$ & $6479(2)$ & $2999(2)$ & $24(1)$ \\
$\mathrm{C}(2 \mathrm{~A})$ & $9328(4)$ & $6556(2)$ & $2422(2)$ & $27(1)$ \\
$\mathrm{C}(3 \mathrm{~A})$ & $9807(4)$ & $5864(2)$ & $2122(2)$ & $24(1)$
\end{tabular}




\begin{tabular}{lrrrr}
$\mathrm{C}(4 \mathrm{~A})$ & $10121(4)$ & $5111(2)$ & $2394(2)$ & $20(1)$ \\
$\mathrm{C}(5 \mathrm{~A})$ & $9939(4)$ & $5085(2)$ & $2979(1)$ & $16(1)$ \\
$\mathrm{C}(6 \mathrm{~A})$ & $10222(3)$ & $4286(2)$ & $3321(1)$ & $15(1)$ \\
$\mathrm{C}(7 \mathrm{~A})$ & $11922(3)$ & $4124(2)$ & $3468(1)$ & $14(1)$ \\
$\mathrm{C}(8 \mathrm{~A})$ & $12544(4)$ & $4840(2)$ & $3836(2)$ & $22(1)$ \\
$\mathrm{C}(9 \mathrm{~A})$ & $12035(4)$ & $3287(2)$ & $3794(1)$ & $22(1)$ \\
$\mathrm{C}(10 \mathrm{~A})$ & $12852(4)$ & $4052(2)$ & $2926(2)$ & $22(1)$ \\
$\mathrm{C}(11 \mathrm{~A})$ & $7333(4)$ & $3893(2)$ & $4618(2)$ & $22(1)$ \\
$\mathrm{C}(12 \mathrm{~A})$ & $5881(4)$ & $3393(3)$ & $4720(2)$ & $36(1)$ \\
$\mathrm{C}(13 \mathrm{~A})$ & $8605(5)$ & $3568(3)$ & $4994(2)$ & $48(1)$ \\
$\mathrm{C}(14 \mathrm{~A})$ & $7040(5)$ & $4830(3)$ & $4701(2)$ & $36(1)$ \\
$\mathrm{Cl}(1 \mathrm{~B})$ & $4622(2)$ & $6318(1)$ & $1310(1)$ & $50(1)$ \\
$\mathrm{S}(1 \mathrm{~B})$ & $6941(1)$ & $8728(1)$ & $3764(1)$ & $17(1)$ \\
$\mathrm{O}(1 \mathrm{~B})$ & $8324(2)$ & $8441(2)$ & $3459(1)$ & $22(1)$ \\
$\mathrm{N}(1 \mathrm{~B})$ & $5367(3)$ & $6564(2)$ & $3180(1)$ & $19(1)$ \\
$\mathrm{N}(2 \mathrm{~B})$ & $5546(3)$ & $8032(2)$ & $3721(1)$ & $17(1)$ \\
$\mathrm{C}(1 \mathrm{~B})$ & $5576(4)$ & $5822(2)$ & $2914(2)$ & $26(1)$ \\
$\mathrm{C}(2 \mathrm{~B})$ & $5351(4)$ & $5715(2)$ & $2341(2)$ & $29(1)$ \\
$\mathrm{C}(3 \mathrm{~B})$ & $4913(4)$ & $6412(2)$ & $2034(2)$ & $27(1)$ \\
$\mathrm{C}(4 \mathrm{~B})$ & $4707(4)$ & $7185(2)$ & $2288(1)$ & $19(1)$ \\
$\mathrm{C}(5 \mathrm{~B})$ & $4926(3)$ & $7226(2)$ & $2871(1)$ & $15(1)$ \\
$\mathrm{C}(6 \mathrm{~B})$ & $4624(3)$ & $8032(2)$ & $3200(1)$ & $13(1)$ \\
$\mathrm{C}(7 \mathrm{~B})$ & $2916(4)$ & $8145(2)$ & $3356(1)$ & $16(1)$ \\
$\mathrm{C}(8 \mathrm{~B})$ & $2351(4)$ & $7395(2)$ & $3701(2)$ & $22(1)$ \\
$\mathrm{C}(9 \mathrm{~B})$ & $2721(4)$ & $8956(2)$ & $3700(2)$ & $24(1)$ \\
$\mathrm{C}(10 \mathrm{~B})$ & $1978(4)$ & $8222(2)$ & $2814(1)$ & $22(1)$ \\
$\mathrm{C}(11 \mathrm{~B})$ & $7363(4)$ & $8547(3)$ & $4523(1)$ & $26(1)$ \\
$\mathrm{C}(12 \mathrm{~B})$ & $7726(5)$ & $7628(3)$ & $4628(2)$ & $45(1)$ \\
$\mathrm{C}(13 \mathrm{~B})$ & $8729(5)$ & $9102(3)$ & $4648(2)$ & $52(1)$ \\
$\mathrm{C}(14 \mathrm{~B})$ & $5999(4)$ & $8841(2)$ & $4867(2)$ & $29(1)$ \\
\hline & & & \\
\hline
\end{tabular}

Table 3. Bond lengths $[\AA]$ and angles $\left[{ }^{\circ}\right]$ for Baran 743 .

\begin{tabular}{llll}
\hline $\mathrm{Cl}(1 \mathrm{~A})-\mathrm{C}(3 \mathrm{~A})$ & $1.744(4)$ & $\mathrm{C}(8 \mathrm{~A})-\mathrm{H}(8 \mathrm{AB})$ & 0.9800 \\
$\mathrm{~S}(1 \mathrm{~A})-\mathrm{O}(1 \mathrm{~A})$ & $1.490(2)$ & $\mathrm{C}(8 \mathrm{~A})-\mathrm{H}(8 \mathrm{AC})$ & 0.9800 \\
$\mathrm{~S}(1 \mathrm{~A})-\mathrm{N}(2 \mathrm{~A})$ & $1.661(3)$ & $\mathrm{C}(9 \mathrm{~A})-\mathrm{H}(9 \mathrm{AA})$ & 0.9800 \\
$\mathrm{~S}(1 \mathrm{~A})-\mathrm{C}(11 \mathrm{~A})$ & $1.846(4)$ & $\mathrm{C}(9 \mathrm{~A})-\mathrm{H}(9 \mathrm{AB})$ & 0.9800 \\
$\mathrm{~N}(1 \mathrm{~A})-\mathrm{C}(1 \mathrm{~A})$ & $1.341(4)$ & $\mathrm{C}(9 \mathrm{~A})-\mathrm{H}(9 \mathrm{AC})$ & 0.9800 \\
$\mathrm{~N}(1 \mathrm{~A})-\mathrm{C}(5 \mathrm{~A})$ & $1.345(4)$ & $\mathrm{C}(10 \mathrm{~A})-\mathrm{H}(10 \mathrm{~A})$ & 0.9800 \\
$\mathrm{~N}(2 \mathrm{~A})-\mathrm{H}(2 \mathrm{~A})$ & $0.87(2)$ & $\mathrm{C}(10 \mathrm{~A})-\mathrm{H}(10 \mathrm{~B})$ & 0.9800 \\
$\mathrm{~N}(2 \mathrm{~A})-\mathrm{C}(6 \mathrm{~A})$ & $1.482(4)$ & $\mathrm{C}(10 \mathrm{~A})-\mathrm{H}(10 \mathrm{C})$ & 0.9800 \\
$\mathrm{C}(1 \mathrm{~A})-\mathrm{H}(1 \mathrm{~A})$ & 0.9500 & $\mathrm{C}(11 \mathrm{~A})-\mathrm{C}(12 \mathrm{~A})$ & $1.528(5)$ \\
$\mathrm{C}(1 \mathrm{~A})-\mathrm{C}(2 \mathrm{~A})$ & $1.376(5)$ & $\mathrm{C}(11 \mathrm{~A})-\mathrm{C}(13 \mathrm{~A})$ & $1.524(5)$ \\
$\mathrm{C}(2 \mathrm{~A})-\mathrm{H}(2 \mathrm{AA})$ & 0.9500 & $\mathrm{C}(11 \mathrm{~A})-\mathrm{C}(14 \mathrm{~A})$ & $1.519(5)$ \\
$\mathrm{C}(2 \mathrm{~A})-\mathrm{C}(3 \mathrm{~A})$ & $1.372(5)$ & $\mathrm{C}(12 \mathrm{~A})-\mathrm{H}(12 \mathrm{~A})$ & 0.9800 \\
$\mathrm{C}(3 \mathrm{~A})-\mathrm{C}(4 \mathrm{~A})$ & $1.383(5)$ & $\mathrm{C}(12 \mathrm{~A})-\mathrm{H}(12 \mathrm{~B})$ & 0.9800 \\
$\mathrm{C}(4 \mathrm{~A})-\mathrm{H}(4 \mathrm{~A})$ & 0.9500 & $\mathrm{C}(12 \mathrm{~A})-\mathrm{H}(12 \mathrm{C})$ & 0.9800 \\
$\mathrm{C}(4 \mathrm{~A})-\mathrm{C}(5 \mathrm{~A})$ & $1.397(5)$ & $\mathrm{C}(13 \mathrm{~A})-\mathrm{H}(13 \mathrm{~A})$ & 0.98000 \\
$\mathrm{C}(5 \mathrm{~A})-\mathrm{C}(6 \mathrm{~A})$ & $1.522(5)$ & $\mathrm{C}(13 \mathrm{~A})-\mathrm{H}(13 \mathrm{~B})$ & 0.9800 \\
$\mathrm{C}(6 \mathrm{~A})-\mathrm{H}(6 \mathrm{~A})$ & 1.0000 & $\mathrm{C}(13 \mathrm{~A})-\mathrm{H}(13 \mathrm{C})$ & 0.9800 \\
$\mathrm{C}(6 \mathrm{~A})-\mathrm{C}(7 \mathrm{~A})$ & $1.566(4)$ & $\mathrm{C}(14 \mathrm{~A})-\mathrm{H}(14 \mathrm{~A})$ & 0.9800 \\
$\mathrm{C}(7 \mathrm{~A})-\mathrm{C}(8 \mathrm{~A})$ & $1.530(4)$ & $\mathrm{C}(14 \mathrm{~A})-\mathrm{H}(14 \mathrm{~B})$ & 0.9800 \\
$\mathrm{C}(7 \mathrm{~A})-\mathrm{C}(9 \mathrm{~A})$ & $1.536(4)$ & $\mathrm{C}(14 \mathrm{~A})-\mathrm{H}(14 \mathrm{C})$ & $1.740(4)$ \\
$\mathrm{C}(7 \mathrm{~A})-\mathrm{C}(10 \mathrm{~A})$ & $1.530(5)$ & $\mathrm{Cl}(1 \mathrm{~B})-\mathrm{C}(3 \mathrm{~B})$ & $1.493(2)$ \\
$\mathrm{C}(8 \mathrm{~A})-\mathrm{H}(8 \mathrm{AA})$ & 0.9800 & $\mathrm{~S}(1 \mathrm{~B})-\mathrm{O}(1 \mathrm{~B})$ & \\
& & &
\end{tabular}




\begin{tabular}{|c|c|}
\hline $\mathrm{S}(1 \mathrm{~B})-\mathrm{N}(2 \mathrm{~B})$ & $1.658(3)$ \\
\hline $\mathrm{S}(1 \mathrm{~B})-\mathrm{C}(11 \mathrm{~B})$ & $1.855(4)$ \\
\hline $\mathrm{N}(1 \mathrm{~B})-\mathrm{C}(1 \mathrm{~B})$ & $1.346(4)$ \\
\hline $\mathrm{N}(1 \mathrm{~B})-\mathrm{C}(5 \mathrm{~B})$ & $1.336(4)$ \\
\hline $\mathrm{N}(2 \mathrm{~B})-\mathrm{H}(2 \mathrm{~B})$ & $0.86(2)$ \\
\hline $\mathrm{N}(2 \mathrm{~B})-\mathrm{C}(6 \mathrm{~B})$ & $1.479(4)$ \\
\hline $\mathrm{C}(1 \mathrm{~B})-\mathrm{H}(1 \mathrm{~B})$ & 0.9500 \\
\hline C(1B)-C(2B) & $1.382(5)$ \\
\hline $\mathrm{C}(2 \mathrm{~B})-\mathrm{H}(2 \mathrm{BA})$ & 0.9500 \\
\hline $\mathrm{C}(2 \mathrm{~B})-\mathrm{C}(3 \mathrm{~B})$ & $1.377(5)$ \\
\hline $\mathrm{C}(3 \mathrm{~B})-\mathrm{C}(4 \mathrm{~B})$ & $1.376(5)$ \\
\hline $\mathrm{C}(4 \mathrm{~B})-\mathrm{H}(4 \mathrm{~B})$ & 0.9500 \\
\hline $\mathrm{C}(4 \mathrm{~B})-\mathrm{C}(5 \mathrm{~B})$ & $1.395(4)$ \\
\hline$C(5 B)-C(6 B)$ & $1.519(4)$ \\
\hline $\mathrm{C}(6 \mathrm{~B})-\mathrm{H}(6 \mathrm{~B})$ & 1.0000 \\
\hline $\mathrm{C}(6 \mathrm{~B})-\mathrm{C}(7 \mathrm{~B})$ & $1.567(4)$ \\
\hline $\mathrm{C}(7 \mathrm{~B})-\mathrm{C}(8 \mathrm{~B})$ & $1.525(4)$ \\
\hline C(7B)-C(9B) & $1.530(4)$ \\
\hline C(7B)-C(10B) & $1.533(4)$ \\
\hline $\mathrm{C}(8 \mathrm{~B})-\mathrm{H}(8 \mathrm{BA})$ & 0.9800 \\
\hline $\mathrm{C}(8 \mathrm{~B})-\mathrm{H}(8 \mathrm{BB})$ & 0.9800 \\
\hline $\mathrm{C}(8 \mathrm{~B})-\mathrm{H}(8 \mathrm{BC})$ & 0.9800 \\
\hline $\mathrm{C}(9 \mathrm{~B})-\mathrm{H}(9 \mathrm{BA})$ & 0.9800 \\
\hline $\mathrm{C}(9 \mathrm{~B})-\mathrm{H}(9 \mathrm{BB})$ & 0.9800 \\
\hline C(9B)-H(9BC) & 0.9800 \\
\hline C(10B)-H(10D) & 0.9800 \\
\hline C(10B)-H(10E) & 0.9800 \\
\hline $\mathrm{C}(10 \mathrm{~B})-\mathrm{H}(10 \mathrm{~F})$ & 0.9800 \\
\hline$C(11 B)-C(12 B)$ & $1.512(6)$ \\
\hline$C(11 B)-C(13 B)$ & $1.524(5)$ \\
\hline$C(11 B)-C(14 B)$ & $1.530(5)$ \\
\hline $\mathrm{C}(12 \mathrm{~B})-\mathrm{H}(12 \mathrm{D})$ & 0.9800 \\
\hline C(12B)-H(12E) & 0.9800 \\
\hline $\mathrm{C}(12 \mathrm{~B})-\mathrm{H}(12 \mathrm{~F})$ & 0.9800 \\
\hline C(13B)-H(13D) & 0.9800 \\
\hline$C(13 B)-H(13 E)$ & 0.9800 \\
\hline $\mathrm{C}(13 \mathrm{~B})-\mathrm{H}(13 \mathrm{~F})$ & 0.9800 \\
\hline C(14B)-H(14D) & 0.9800 \\
\hline $\mathrm{C}(14 \mathrm{~B})-\mathrm{H}(14 \mathrm{E})$ & 0.9800 \\
\hline $\mathrm{C}(14 \mathrm{~B})-\mathrm{H}(14 \mathrm{~F})$ & 0.9800 \\
\hline $\mathrm{O}(1 \mathrm{~A})-\mathrm{S}(1 \mathrm{~A})-\mathrm{N}(2 \mathrm{~A})$ & $112.37(14)$ \\
\hline $\mathrm{O}(1 \mathrm{~A})-\mathrm{S}(1 \mathrm{~A})-\mathrm{C}(11 \mathrm{~A})$ & $106.02(15)$ \\
\hline $\mathrm{N}(2 \mathrm{~A})-\mathrm{S}(1 \mathrm{~A})-\mathrm{C}(11 \mathrm{~A})$ & $96.44(15)$ \\
\hline $\mathrm{C}(1 \mathrm{~A})-\mathrm{N}(1 \mathrm{~A})-\mathrm{C}(5 \mathrm{~A})$ & $118.3(3)$ \\
\hline $\mathrm{S}(1 \mathrm{~A})-\mathrm{N}(2 \mathrm{~A})-\mathrm{H}(2 \mathrm{~A})$ & 112(2) \\
\hline $\mathrm{C}(6 \mathrm{~A})-\mathrm{N}(2 \mathrm{~A})-\mathrm{S}(1 \mathrm{~A})$ & $116.5(2)$ \\
\hline $\mathrm{C}(6 \mathrm{~A})-\mathrm{N}(2 \mathrm{~A})-\mathrm{H}(2 \mathrm{~A})$ & $109(2)$ \\
\hline $\mathrm{N}(1 \mathrm{~A})-\mathrm{C}(1 \mathrm{~A})-\mathrm{H}(1 \mathrm{~A})$ & 118.4 \\
\hline $\mathrm{N}(1 \mathrm{~A})-\mathrm{C}(1 \mathrm{~A})-\mathrm{C}(2 \mathrm{~A})$ & $123.2(3)$ \\
\hline $\mathrm{C}(2 \mathrm{~A})-\mathrm{C}(1 \mathrm{~A})-\mathrm{H}(1 \mathrm{~A})$ & 118.4 \\
\hline $\mathrm{C}(1 \mathrm{~A})-\mathrm{C}(2 \mathrm{~A})-\mathrm{H}(2 \mathrm{AA})$ & 121.0 \\
\hline $\mathrm{C}(3 \mathrm{~A})-\mathrm{C}(2 \mathrm{~A})-\mathrm{C}(1 \mathrm{~A})$ & $118.0(3)$ \\
\hline $\mathrm{C}(3 \mathrm{~A})-\mathrm{C}(2 \mathrm{~A})-\mathrm{H}(2 \mathrm{AA})$ & 121.0 \\
\hline $\mathrm{C}(2 \mathrm{~A})-\mathrm{C}(3 \mathrm{~A})-\mathrm{Cl}(1 \mathrm{~A})$ & $120.0(3)$ \\
\hline $\mathrm{C}(2 \mathrm{~A})-\mathrm{C}(3 \mathrm{~A})-\mathrm{C}(4 \mathrm{~A})$ & $120.7(3)$ \\
\hline
\end{tabular}

$\begin{array}{ll} & \\ \text { C(4A)-C(3A)-Cl(1A) } & 119.3(3) \\ \text { C(3A)-C(4A)-H(4A) } & 121.2 \\ \text { C(3A)-C(4A)-C(5A) } & 117.6(3) \\ \text { C(5A)-C(4A)-H(4A) } & 121.2 \\ \text { N(1A)-C(5A)-C(4A) } & 122.2(3) \\ \text { N(1A)-C(5A)-C(6A) } & 115.6(3) \\ \text { C(4A)-C(5A)-C(6A) } & 122.2(3) \\ \text { N(2A)-C(6A)-C(5A) } & 109.2(2) \\ \text { N(2A)-C(6A)-H(6A) } & 107.7 \\ \text { N(2A)-C(6A)-C(7A) } & 110.0(2) \\ \text { C(5A)-C(6A)-H(6A) } & 107.7 \\ \text { C(5A)-C(6A)-C(7A) } & 114.4(3) \\ \text { C(7A)-C(6A)-H(6A) } & 107.7 \\ \text { C(8A)-C(7A)-C(6A) } & 110.6(2) \\ \text { C(8A)-C(7A)-C(9A) } & 109.3(3) \\ \text { C(9A)-C(7A)-C(6A) } & 108.4(3) \\ \text { C(10A)-C(7A)-C(6A) } & 110.0(3) \\ \text { C(10A)-C(7A)-C(8A) } & 109.8(3) \\ \text { C(10A)-C(7A)-C(9A) } & 108.8(3) \\ \text { C(7A)-C(8A)-H(8AA) } & 109.5 \\ \text { C(7A)-C(8A)-H(8AB) } & 109.5 \\ \text { C(7A)-C(8A)-H(8AC) } & 109.5 \\ \text { H(8AA)-C(8A)-H(8AB) } & 109.5 \\ \text { H(8AA)-C(8A)-H(8AC) } & 109.5 \\ \text { H(8AB)-C(8A)-H(8AC) } & 109.5 \\ \text { C(7A)-C(9A)-H(9AA) } & 109.5 \\ \text { C(7A)-C(9A)-H(9AB) } & 109.5 \\ \text { C(7A)-C(9A)-H(9AC) } & 109.5 \\ \text { H(9AA)-C(9A)-H(9AB) } & 109.5 \\ \text { H(9AA)-C(9A)-H(9AC) } & 109.5 \\ \text { H(9AB)-C(9A)-H(9AC) } & 109.5 \\ \text { C(7A)-C(10A)-H(10A) } & 109.5 \\ \text { C(7A)-C(10A)-H(10B) } & 109.5 \\ \text { C(7A)-C(10A)-H(10C) } & 109.5 \\ \text { H(10A)-C(10A)-H(10B) } & 109.5 \\ \text { H(10A)-C(10A)-H(10C) } & 109.5 \\ \text { H(10B)-C(10A)-H(10C) } & 109.5 \\ \text { C(12A)-C(11A)-S(1A) } & 104.6(2) \\ \text { C(13A)-C(11A)-S(1A) } & 108.4(3) \\ \text { C(13A)-C(11A)-C(12A) } & 110.8(3) \\ \text { C(14A)-C(11A)-S(1A) } & 110.4(3) \\ \text { C(14A)-C(11A)-C(12A) } & 110.0(3) \\ \text { C(14A)-C(11A)-C(13A) } & 112.4(3) \\ \text { C(11A)-C(12A)-H(12A) } & 109.5 \\ \text { C(11A)-C(12A)-H(12B) } & 109.5 \\ \text { C(11A)-C(12A)-H(12C) } & 109.5 \\ \text { H(12A)-C(12A)-H(12B) } & 109.5 \\ \text { H(12A)-C(12A)-H(12C) } & 109.5 \\ \text { H(12B)-C(12A)-H(12C) } & 109.5 \\ \text { C(11A)-C(13A)-H(13A) } & 109.5 \\ \text { C(11A)-C(13A)-H(13B) } & 109.5 \\ \text { C(11A)-C(13A)-H(13C) } & 109.5 \\ \text { H(13A)-C(13A)-H(13B) } & 109.5 \\ \text { H(13A)-C(13A)-H(13C) } & 109.5 \\ \text { H(13B)-C(13A)-H(13C) } & 109.5 \\ \text { C(11A)-C(14A)-H(14A) } & 109.5 \\ & \\ & \\ \end{array}$




\begin{tabular}{|c|c|c|c|}
\hline $\mathrm{C}(11 \mathrm{~A})-\mathrm{C}(14 \mathrm{~A})-\mathrm{H}(14 \mathrm{~B})$ & 109.5 & $\mathrm{C}(7 \mathrm{~B})-\mathrm{C}(8 \mathrm{~B})-\mathrm{H}(8 \mathrm{BC})$ & 109.5 \\
\hline $\mathrm{C}(11 \mathrm{~A})-\mathrm{C}(14 \mathrm{~A})-\mathrm{H}(14 \mathrm{C})$ & 109.5 & $\mathrm{H}(8 \mathrm{BA})-\mathrm{C}(8 \mathrm{~B})-\mathrm{H}(8 \mathrm{BB})$ & 109.5 \\
\hline $\mathrm{H}(14 \mathrm{~A})-\mathrm{C}(14 \mathrm{~A})-\mathrm{H}(14 \mathrm{~B})$ & 109.5 & $\mathrm{H}(8 \mathrm{BA})-\mathrm{C}(8 \mathrm{~B})-\mathrm{H}(8 \mathrm{BC})$ & 109.5 \\
\hline $\mathrm{H}(14 \mathrm{~A})-\mathrm{C}(14 \mathrm{~A})-\mathrm{H}(14 \mathrm{C})$ & 109.5 & $\mathrm{H}(8 \mathrm{BB})-\mathrm{C}(8 \mathrm{~B})-\mathrm{H}(8 \mathrm{BC})$ & 109.5 \\
\hline $\mathrm{H}(14 \mathrm{~B})-\mathrm{C}(14 \mathrm{~A})-\mathrm{H}(14 \mathrm{C})$ & 109.5 & $\mathrm{C}(7 \mathrm{~B})-\mathrm{C}(9 \mathrm{~B})-\mathrm{H}(9 \mathrm{BA})$ & 109.5 \\
\hline $\mathrm{O}(1 \mathrm{~B})-\mathrm{S}(1 \mathrm{~B})-\mathrm{N}(2 \mathrm{~B})$ & $112.25(14)$ & $\mathrm{C}(7 \mathrm{~B})-\mathrm{C}(9 \mathrm{~B})-\mathrm{H}(9 \mathrm{BB})$ & 109.5 \\
\hline $\mathrm{O}(1 \mathrm{~B})-\mathrm{S}(1 \mathrm{~B})-\mathrm{C}(11 \mathrm{~B})$ & $104.86(15)$ & C(7B)-C(9B)-H(9BC) & 109.5 \\
\hline$N(2 B)-S(1 B)-C(11 B)$ & $96.18(15)$ & $\mathrm{H}(9 \mathrm{BA})-\mathrm{C}(9 \mathrm{~B})-\mathrm{H}(9 \mathrm{BB})$ & 109.5 \\
\hline $\mathrm{C}(5 \mathrm{~B})-\mathrm{N}(1 \mathrm{~B})-\mathrm{C}(1 \mathrm{~B})$ & $117.9(3)$ & $H(9 B A)-C(9 B)-H(9 B C)$ & 109.5 \\
\hline $\mathrm{S}(1 \mathrm{~B})-\mathrm{N}(2 \mathrm{~B})-\mathrm{H}(2 \mathrm{~B})$ & $112(2)$ & $\mathrm{H}(9 \mathrm{BB})-\mathrm{C}(9 \mathrm{~B})-\mathrm{H}(9 \mathrm{BC})$ & 109.5 \\
\hline $\mathrm{C}(6 \mathrm{~B})-\mathrm{N}(2 \mathrm{~B})-\mathrm{S}(1 \mathrm{~B})$ & $117.5(2)$ & $\mathrm{C}(7 \mathrm{~B})-\mathrm{C}(10 \mathrm{~B})-\mathrm{H}(10 \mathrm{D})$ & 109.5 \\
\hline $\mathrm{C}(6 \mathrm{~B})-\mathrm{N}(2 \mathrm{~B})-\mathrm{H}(2 \mathrm{~B})$ & $111(2)$ & $\mathrm{C}(7 \mathrm{~B})-\mathrm{C}(10 \mathrm{~B})-\mathrm{H}(10 \mathrm{E})$ & 109.5 \\
\hline $\mathrm{N}(1 \mathrm{~B})-\mathrm{C}(1 \mathrm{~B})-\mathrm{H}(1 \mathrm{~B})$ & 118.4 & $\mathrm{C}(7 \mathrm{~B})-\mathrm{C}(10 \mathrm{~B})-\mathrm{H}(10 \mathrm{~F})$ & 109.5 \\
\hline $\mathrm{N}(1 \mathrm{~B})-\mathrm{C}(1 \mathrm{~B})-\mathrm{C}(2 \mathrm{~B})$ & $123.1(3)$ & $\mathrm{H}(10 \mathrm{D})-\mathrm{C}(10 \mathrm{~B})-\mathrm{H}(10 \mathrm{E})$ & 109.5 \\
\hline $\mathrm{C}(2 \mathrm{~B})-\mathrm{C}(1 \mathrm{~B})-\mathrm{H}(1 \mathrm{~B})$ & 118.4 & $\mathrm{H}(10 \mathrm{D})-\mathrm{C}(10 \mathrm{~B})-\mathrm{H}(10 \mathrm{~F})$ & 109.5 \\
\hline $\mathrm{C}(1 \mathrm{~B})-\mathrm{C}(2 \mathrm{~B})-\mathrm{H}(2 \mathrm{BA})$ & 121.3 & $\mathrm{H}(10 \mathrm{E})-\mathrm{C}(10 \mathrm{~B})-\mathrm{H}(10 \mathrm{~F})$ & 109.5 \\
\hline $\mathrm{C}(3 \mathrm{~B})-\mathrm{C}(2 \mathrm{~B})-\mathrm{C}(1 \mathrm{~B})$ & $117.4(3)$ & $\mathrm{C}(12 \mathrm{~B})-\mathrm{C}(11 \mathrm{~B})-\mathrm{S}(1 \mathrm{~B})$ & $110.5(2)$ \\
\hline $\mathrm{C}(3 \mathrm{~B})-\mathrm{C}(2 \mathrm{~B})-\mathrm{H}(2 \mathrm{BA})$ & 121.3 & $\mathrm{C}(12 \mathrm{~B})-\mathrm{C}(11 \mathrm{~B})-\mathrm{C}(13 \mathrm{~B})$ & $110.8(4)$ \\
\hline $\mathrm{C}(2 \mathrm{~B})-\mathrm{C}(3 \mathrm{~B})-\mathrm{Cl}(1 \mathrm{~B})$ & $119.5(3)$ & $C(12 B)-C(11 B)-C(14 B)$ & $111.9(3)$ \\
\hline $\mathrm{C}(4 \mathrm{~B})-\mathrm{C}(3 \mathrm{~B})-\mathrm{Cl}(1 \mathrm{~B})$ & $119.2(3)$ & $C(13 B)-C(11 B)-S(1 B)$ & $105.0(3)$ \\
\hline $\mathrm{C}(4 \mathrm{~B})-\mathrm{C}(3 \mathrm{~B})-\mathrm{C}(2 \mathrm{~B})$ & $121.4(3)$ & $\mathrm{C}(13 \mathrm{~B})-\mathrm{C}(11 \mathrm{~B})-\mathrm{C}(14 \mathrm{~B})$ & $110.4(3)$ \\
\hline $\mathrm{C}(3 \mathrm{~B})-\mathrm{C}(4 \mathrm{~B})-\mathrm{H}(4 \mathrm{~B})$ & 121.5 & $\mathrm{C}(14 \mathrm{~B})-\mathrm{C}(11 \mathrm{~B})-\mathrm{S}(1 \mathrm{~B})$ & $108.1(2)$ \\
\hline$C(3 B)-C(4 B)-C(5 B)$ & $117.0(3)$ & $\mathrm{C}(11 \mathrm{~B})-\mathrm{C}(12 \mathrm{~B})-\mathrm{H}(12 \mathrm{D})$ & 109.5 \\
\hline $\mathrm{C}(5 \mathrm{~B})-\mathrm{C}(4 \mathrm{~B})-\mathrm{H}(4 \mathrm{~B})$ & 121.5 & $\mathrm{C}(11 \mathrm{~B})-\mathrm{C}(12 \mathrm{~B})-\mathrm{H}(12 \mathrm{E})$ & 109.5 \\
\hline $\mathrm{N}(1 \mathrm{~B})-\mathrm{C}(5 \mathrm{~B})-\mathrm{C}(4 \mathrm{~B})$ & $123.1(3)$ & $\mathrm{C}(11 \mathrm{~B})-\mathrm{C}(12 \mathrm{~B})-\mathrm{H}(12 \mathrm{~F})$ & 109.5 \\
\hline$N(1 B)-C(5 B)-C(6 B)$ & $115.5(3)$ & $\mathrm{H}(12 \mathrm{D})-\mathrm{C}(12 \mathrm{~B})-\mathrm{H}(12 \mathrm{E})$ & 109.5 \\
\hline $\mathrm{C}(4 \mathrm{~B})-\mathrm{C}(5 \mathrm{~B})-\mathrm{C}(6 \mathrm{~B})$ & $121.4(3)$ & $\mathrm{H}(12 \mathrm{D})-\mathrm{C}(12 \mathrm{~B})-\mathrm{H}(12 \mathrm{~F})$ & 109.5 \\
\hline $\mathrm{N}(2 \mathrm{~B})-\mathrm{C}(6 \mathrm{~B})-\mathrm{C}(5 \mathrm{~B})$ & $109.3(2)$ & $\mathrm{H}(12 \mathrm{E})-\mathrm{C}(12 \mathrm{~B})-\mathrm{H}(12 \mathrm{~F})$ & 109.5 \\
\hline $\mathrm{N}(2 \mathrm{~B})-\mathrm{C}(6 \mathrm{~B})-\mathrm{H}(6 \mathrm{~B})$ & 108.4 & $\mathrm{C}(11 \mathrm{~B})-\mathrm{C}(13 \mathrm{~B})-\mathrm{H}(13 \mathrm{D})$ & 109.5 \\
\hline $\mathrm{N}(2 \mathrm{~B})-\mathrm{C}(6 \mathrm{~B})-\mathrm{C}(7 \mathrm{~B})$ & $109.6(2)$ & $\mathrm{C}(11 \mathrm{~B})-\mathrm{C}(13 \mathrm{~B})-\mathrm{H}(13 \mathrm{E})$ & 109.5 \\
\hline $\mathrm{C}(5 \mathrm{~B})-\mathrm{C}(6 \mathrm{~B})-\mathrm{H}(6 \mathrm{~B})$ & 108.4 & $\mathrm{C}(11 \mathrm{~B})-\mathrm{C}(13 \mathrm{~B})-\mathrm{H}(13 \mathrm{~F})$ & 109.5 \\
\hline$C(5 B)-C(6 B)-C(7 B)$ & $112.7(3)$ & $\mathrm{H}(13 \mathrm{D})-\mathrm{C}(13 \mathrm{~B})-\mathrm{H}(13 \mathrm{E})$ & 109.5 \\
\hline $\mathrm{C}(7 \mathrm{~B})-\mathrm{C}(6 \mathrm{~B})-\mathrm{H}(6 \mathrm{~B})$ & 108.4 & $\mathrm{H}(13 \mathrm{D})-\mathrm{C}(13 \mathrm{~B})-\mathrm{H}(13 \mathrm{~F})$ & 109.5 \\
\hline$C(8 B)-C(7 B)-C(6 B)$ & $110.7(3)$ & $\mathrm{H}(13 \mathrm{E})-\mathrm{C}(13 \mathrm{~B})-\mathrm{H}(13 \mathrm{~F})$ & 109.5 \\
\hline $\mathrm{C}(8 \mathrm{~B})-\mathrm{C}(7 \mathrm{~B})-\mathrm{C}(9 \mathrm{~B})$ & $109.3(3)$ & $\mathrm{C}(11 \mathrm{~B})-\mathrm{C}(14 \mathrm{~B})-\mathrm{H}(14 \mathrm{D})$ & 109.5 \\
\hline $\mathrm{C}(8 \mathrm{~B})-\mathrm{C}(7 \mathrm{~B})-\mathrm{C}(10 \mathrm{~B})$ & $109.4(3)$ & $\mathrm{C}(11 \mathrm{~B})-\mathrm{C}(14 \mathrm{~B})-\mathrm{H}(14 \mathrm{E})$ & 109.5 \\
\hline$C(9 B)-C(7 B)-C(6 B)$ & $109.3(3)$ & $\mathrm{C}(11 \mathrm{~B})-\mathrm{C}(14 \mathrm{~B})-\mathrm{H}(14 \mathrm{~F})$ & 109.5 \\
\hline$C(9 B)-C(7 B)-C(10 B)$ & $108.5(3)$ & $\mathrm{H}(14 \mathrm{D})-\mathrm{C}(14 \mathrm{~B})-\mathrm{H}(14 \mathrm{E})$ & 109.5 \\
\hline$C(10 B)-C(7 B)-C(6 B)$ & $109.5(3)$ & $\mathrm{H}(14 \mathrm{D})-\mathrm{C}(14 \mathrm{~B})-\mathrm{H}(14 \mathrm{~F})$ & 109.5 \\
\hline $\mathrm{C}(7 \mathrm{~B})-\mathrm{C}(8 \mathrm{~B})-\mathrm{H}(8 \mathrm{BA})$ & 109.5 & $\mathrm{H}(14 \mathrm{E})-\mathrm{C}(14 \mathrm{~B})-\mathrm{H}(14 \mathrm{~F})$ & 109.5 \\
\hline $\mathrm{C}(7 \mathrm{~B})-\mathrm{C}(8 \mathrm{~B})-\mathrm{H}(8 \mathrm{BB})$ & 109.5 & & \\
\hline
\end{tabular}

Symmetry transformations used to generate equivalent atoms:

Table 4. Anisotropic displacement parameters $\left(\AA^{2} \times 10^{3}\right)$ for Baran743. The anisotropic displacement factor exponent takes the form: $-2 \pi^{2}\left[h^{2} a^{* 2} U^{11}+\ldots+2 h k a^{*} b^{*} U^{12}\right]$

\begin{tabular}{lcccccc}
\hline & $\mathrm{U}^{11}$ & $\mathrm{U}^{22}$ & $\mathrm{U}^{33}$ & $\mathrm{U}^{23}$ & $\mathrm{U}^{13}$ & $\mathrm{U}^{12}$ \\
\hline $\mathrm{Cl}(1 \mathrm{~A})$ & $71(1)$ & $32(1)$ & $27(1)$ & $12(1)$ & $-4(1)$ & $-3(1)$ \\
$\mathrm{S}(1 \mathrm{~A})$ & $14(1)$ & $16(1)$ & $16(1)$ & $2(1)$ & $-2(1)$ & $-2(1)$ \\
$\mathrm{O}(1 \mathrm{~A})$ & $17(1)$ & $29(1)$ & $18(1)$ & $4(1)$ & $-4(1)$ & $-1(1)$ \\
$\mathrm{N}(1 \mathrm{~A})$ & $12(1)$ & $18(2)$ & $29(2)$ & $-4(1)$ & $-5(1)$ & $1(1)$ \\
$\mathrm{N}(2 \mathrm{~A})$ & $13(1)$ & $15(2)$ & $21(2)$ & $-4(1)$ & $1(1)$ & $0(1)$ \\
$\mathrm{C}(1 \mathrm{~A})$ & $18(2)$ & $13(2)$ & $40(2)$ & $-4(2)$ & $-6(2)$ & $3(1)$
\end{tabular}




\begin{tabular}{lcccccc}
$\mathrm{C}(2 \mathrm{~A})$ & $26(2)$ & $16(2)$ & $39(2)$ & $5(2)$ & $-11(2)$ & $-1(2)$ \\
$\mathrm{C}(3 \mathrm{~A})$ & $25(2)$ & $25(2)$ & $23(2)$ & $6(2)$ & $-6(2)$ & $-4(2)$ \\
$\mathrm{C}(4 \mathrm{~A})$ & $17(2)$ & $17(2)$ & $25(2)$ & $0(1)$ & $-3(2)$ & $-2(1)$ \\
$\mathrm{C}(5 \mathrm{~A})$ & $10(2)$ & $15(2)$ & $24(2)$ & $-2(1)$ & $-3(1)$ & $-1(1)$ \\
$\mathrm{C}(6 \mathrm{~A})$ & $13(2)$ & $15(2)$ & $17(2)$ & $-3(1)$ & $-1(1)$ & $0(1)$ \\
$\mathrm{C}(7 \mathrm{~A})$ & $11(2)$ & $13(2)$ & $19(2)$ & $-4(1)$ & $-4(1)$ & $3(1)$ \\
$\mathrm{C}(8 \mathrm{~A})$ & $14(2)$ & $23(2)$ & $28(2)$ & $-7(2)$ & $-6(2)$ & $2(1)$ \\
$\mathrm{C}(9 \mathrm{~A})$ & $22(2)$ & $20(2)$ & $24(2)$ & $-2(2)$ & $-9(2)$ & $3(1)$ \\
$\mathrm{C}(10 \mathrm{~A})$ & $15(2)$ & $26(2)$ & $25(2)$ & $-1(2)$ & $1(2)$ & $4(2)$ \\
$\mathrm{C}(11 \mathrm{~A})$ & $20(2)$ & $35(2)$ & $13(2)$ & $1(2)$ & $-2(1)$ & $-4(2)$ \\
$\mathrm{C}(12 \mathrm{~A})$ & $31(2)$ & $55(3)$ & $21(2)$ & $6(2)$ & $3(2)$ & $-10(2)$ \\
$\mathrm{C}(13 \mathrm{~A})$ & $32(2)$ & $90(4)$ & $22(2)$ & $16(2)$ & $-8(2)$ & $6(3)$ \\
$\mathrm{C}(14 \mathrm{~A})$ & $26(2)$ & $51(3)$ & $31(2)$ & $-16(2)$ & $6(2)$ & $0(2)$ \\
$\mathrm{Cl}(1 \mathrm{~B})$ & $86(1)$ & $41(1)$ & $22(1)$ & $-14(1)$ & $18(1)$ & $-18(1)$ \\
$\mathrm{S}(1 \mathrm{~B})$ & $14(1)$ & $18(1)$ & $19(1)$ & $-5(1)$ & $2(1)$ & $-3(1)$ \\
$\mathrm{O}(1 \mathrm{~B})$ & $15(1)$ & $32(1)$ & $20(1)$ & $-3(1)$ & $3(1)$ & $0(1)$ \\
$\mathrm{N}(1 \mathrm{~B})$ & $14(2)$ & $14(2)$ & $28(2)$ & $3(1)$ & $0(1)$ & $0(1)$ \\
$\mathrm{N}(2 \mathrm{~B})$ & $17(1)$ & $16(2)$ & $18(2)$ & $2(1)$ & $-4(1)$ & $-2(1)$ \\
$\mathrm{C}(1 \mathrm{~B})$ & $19(2)$ & $16(2)$ & $43(2)$ & $0(2)$ & $7(2)$ & $2(1)$ \\
$\mathrm{C}(2 \mathrm{~B})$ & $32(2)$ & $18(2)$ & $38(2)$ & $-11(2)$ & $19(2)$ & $-4(2)$ \\
$\mathrm{C}(3 \mathrm{~B})$ & $30(2)$ & $27(2)$ & $22(2)$ & $-8(2)$ & $13(2)$ & $-12(2)$ \\
$\mathrm{C}(4 \mathrm{~B})$ & $19(2)$ & $18(2)$ & $22(2)$ & $-1(1)$ & $7(2)$ & $-4(1)$ \\
$\mathrm{C}(5 \mathrm{~B})$ & $8(2)$ & $19(2)$ & $18(2)$ & $1(1)$ & $3(1)$ & $-2(1)$ \\
$\mathrm{C}(6 \mathrm{~B})$ & $14(2)$ & $14(2)$ & $11(2)$ & $1(1)$ & $0(1)$ & $-2(1)$ \\
$\mathrm{C}(7 \mathrm{~B})$ & $16(2)$ & $16(2)$ & $16(2)$ & $4(1)$ & $3(1)$ & $0(1)$ \\
$\mathrm{C}(8 \mathrm{~B})$ & $18(2)$ & $28(2)$ & $21(2)$ & $7(2)$ & $2(2)$ & $-3(1)$ \\
$\mathrm{C}(9 \mathrm{~B})$ & $22(2)$ & $26(2)$ & $25(2)$ & $-3(2)$ & $11(2)$ & $2(2)$ \\
$\mathrm{C}(10 \mathrm{~B})$ & $16(2)$ & $28(2)$ & $21(2)$ & $4(2)$ & $2(2)$ & $3(2)$ \\
$\mathrm{C}(11 \mathrm{~B})$ & $19(2)$ & $45(2)$ & $14(2)$ & $-11(2)$ & $0(1)$ & $-2(2)$ \\
$\mathrm{C}(12 \mathrm{~B})$ & $54(3)$ & $65(3)$ & $15(2)$ & $0(2)$ & $-10(2)$ & $26(2)$ \\
$\mathrm{C}(13 \mathrm{~B})$ & $28(2)$ & $102(4)$ & $26(2)$ & $-26(3)$ & $3(2)$ & $-24(3)$ \\
$\mathrm{C}(14 \mathrm{~B})$ & $22(2)$ & $41(2)$ & $23(2)$ & $-9(2)$ & $4(2)$ & $-5(2)$ \\
\hline & & & & & & \\
\hline & & & & & & \\
& & & & & \\
\end{tabular}

Table 5. Hydrogen coordinates (x $\left.10^{4}\right)$ and isotropic displacement parameters $\left(\AA^{2} \times 10^{3}\right)$ for Baran743.

\begin{tabular}{lclll}
\hline & \multicolumn{1}{c}{$\mathrm{x}$} & \multicolumn{1}{c}{$\mathrm{y}$} & \multicolumn{1}{c}{$\mathrm{z}$} & $\mathrm{U}(\mathrm{eq})$ \\
\hline & & & & \\
$\mathrm{H}(2 \mathrm{~A})$ & $9050(40)$ & $4838(15)$ & $3910(14)$ & $18(10)$ \\
$\mathrm{H}(1 \mathrm{~A})$ & 8868 & 6958 & 3209 & 29 \\
$\mathrm{H}(2 \mathrm{AA})$ & 9101 & 7073 & 2237 & 32 \\
$\mathrm{H}(4 \mathrm{~A})$ & 10450 & 4629 & 2189 & 24 \\
$\mathrm{H}(6 \mathrm{~A})$ & 9859 & 3796 & 3091 & 18 \\
$\mathrm{H}(8 \mathrm{AA})$ & 12458 & 5376 & 3631 & 33 \\
$\mathrm{H}(8 \mathrm{AB})$ & 13607 & 4729 & 3923 & 33 \\
$\mathrm{H}(8 \mathrm{AC})$ & 11963 & 4872 & 4188 & 33 \\
$\mathrm{H}(9 \mathrm{AA})$ & 11526 & 3344 & 4160 & 33 \\
$\mathrm{H}(9 \mathrm{AB})$ & 13100 & 3146 & 3855 & 33 \\
$\mathrm{H}(9 \mathrm{AC})$ & 11551 & 2837 & 3575 & 33 \\
$\mathrm{H}(10 \mathrm{~A})$ & 12412 & 3617 & 2682 & 33 \\
$\mathrm{H}(10 \mathrm{~B})$ & 13894 & 3897 & 3020 & 33 \\
$\mathrm{H}(10 \mathrm{C})$ & 12846 & 4595 & 2728 & 33 \\
$\mathrm{H}(12 \mathrm{~A})$ & 5084 & 3606 & 4471 & 53 \\
$\mathrm{H}(12 \mathrm{~B})$ & 5567 & 3459 & 5115 & 53 \\
& & & &
\end{tabular}




\begin{tabular}{lllll}
$\mathrm{H}(12 \mathrm{C})$ & 6062 & 2794 & 4640 & 53 \\
$\mathrm{H}(13 \mathrm{~A})$ & 8792 & 2971 & 4911 & 72 \\
$\mathrm{H}(13 \mathrm{~B})$ & 8316 & 3630 & 5392 & 72 \\
$\mathrm{H}(13 \mathrm{C})$ & 9525 & 3895 & 4921 & 72 \\
$\mathrm{H}(14 \mathrm{~A})$ & 7999 & 5138 & 4686 & 54 \\
$\mathrm{H}(14 \mathrm{~B})$ & 6560 & 4922 & 5069 & 54 \\
$\mathrm{H}(14 \mathrm{C})$ & 6371 & 5034 & 4401 & 54 \\
$\mathrm{H}(2 \mathrm{~B})$ & $5850(40)$ & $7528(15)$ & $3802(14)$ & $15(9)$ \\
$\mathrm{H}(1 \mathrm{~B})$ & 5894 & 5349 & 3131 & 31 \\
$\mathrm{H}(2 \mathrm{BA})$ & 5493 & 5181 & 2165 & 35 \\
$\mathrm{H}(4 \mathrm{~B})$ & 4427 & 7670 & 2076 & 23 \\
$\mathrm{H}(6 \mathrm{~B})$ & 4941 & 8523 & 2962 & 16 \\
$\mathrm{H}(8 \mathrm{BA})$ & 2367 & 6885 & 3467 & 33 \\
$\mathrm{H}(8 \mathrm{BB})$ & 1316 & 7505 & 3828 & 33 \\
$\mathrm{H}(8 \mathrm{BC})$ & 3008 & 7314 & 4030 & 33 \\
$\mathrm{H}(9 \mathrm{BA})$ & 3237 & 8896 & 4064 & 37 \\
$\mathrm{H}(9 \mathrm{BB})$ & 1643 & 9060 & 3764 & 37 \\
$\mathrm{H}(9 \mathrm{BC})$ & 3158 & 9432 & 3492 & 37 \\
$\mathrm{H}(10 \mathrm{D})$ & 2375 & 8684 & 2582 & 32 \\
$\mathrm{H}(10 \mathrm{E})$ & 923 & 8339 & 2912 & 32 \\
$\mathrm{H}(10 \mathrm{~F})$ & 2036 & 7692 & 2601 & 32 \\
$\mathrm{H}(12 \mathrm{D})$ & 6801 & 7291 & 4594 & 67 \\
$\mathrm{H}(12 \mathrm{E})$ & 8146 & 7562 & 5008 & 67 \\
$\mathrm{H}(12 \mathrm{~F})$ & 8466 & 7433 & 4348 & 67 \\
$\mathrm{H}(13 \mathrm{D})$ & 9586 & 8917 & 4417 & 78 \\
$\mathrm{H}(13 \mathrm{E})$ & 8993 & 9055 & 5049 & 78 \\
$\mathrm{H}(13 \mathrm{~F})$ & 8488 & 9691 & 4558 & 78 \\
$\mathrm{H}(14 \mathrm{D})$ & 5757 & 9427 & 4769 & 43 \\
$\mathrm{H}(14 \mathrm{E})$ & 6235 & 8805 & 5271 & 43 \\
$\mathrm{H}(14 \mathrm{~F})$ & 5129 & 8479 & 4783 & 43 \\
& & & & \\
\hline
\end{tabular}




\section{Compound 9}

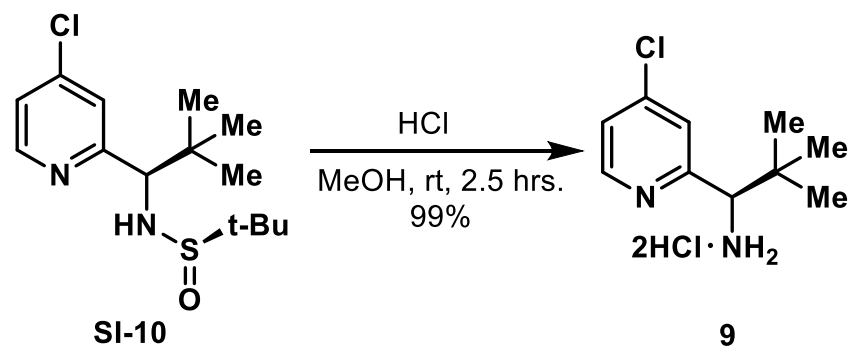

See GP-3. Prepared on $41 \mathrm{mmol}$ scale to afford 9 (10.9 g, 40.4 mmol, $99 \%$ yield) as deliquescent light brown crystals.

Physical state: deliquescent light brown crystals

m.p.: $236-238{ }^{\circ} \mathrm{C}$

$[\boldsymbol{\alpha}]_{D}^{20.0}=-3.6(c=1.0, \mathrm{MeOH})$

${ }^{1}$ H NMR (600 MHz, DMSO-d6): $\delta 8.60$ (d, $\left.J=5.4 \mathrm{~Hz}, 1 \mathrm{H}\right), 8.56$ (s, 3H), 7.74 (d, $J=1.8 \mathrm{~Hz}$, $1 \mathrm{H}), 7.58(\mathrm{dd}, J=5.4,2.0 \mathrm{~Hz}, 1 \mathrm{H}), 4.23(\mathrm{q}, J=5.6 \mathrm{~Hz}, 1 \mathrm{H}), 0.94(\mathrm{~s}, 9 \mathrm{H})$.

${ }^{13}$ C NMR (150 MHz, DMSO-d6): $\delta$ 157.1, 150.0, 143.1, 124.4, 123.6, 62.0, 34.1, 26.2.

HRMS (m/z): calculated for $\mathrm{C}_{10} \mathrm{H}_{16} \mathrm{ClN}_{2}[\mathrm{M}+\mathrm{H}]^{+}$: 199.0997, found 199.1002.

\section{Compound 10d}

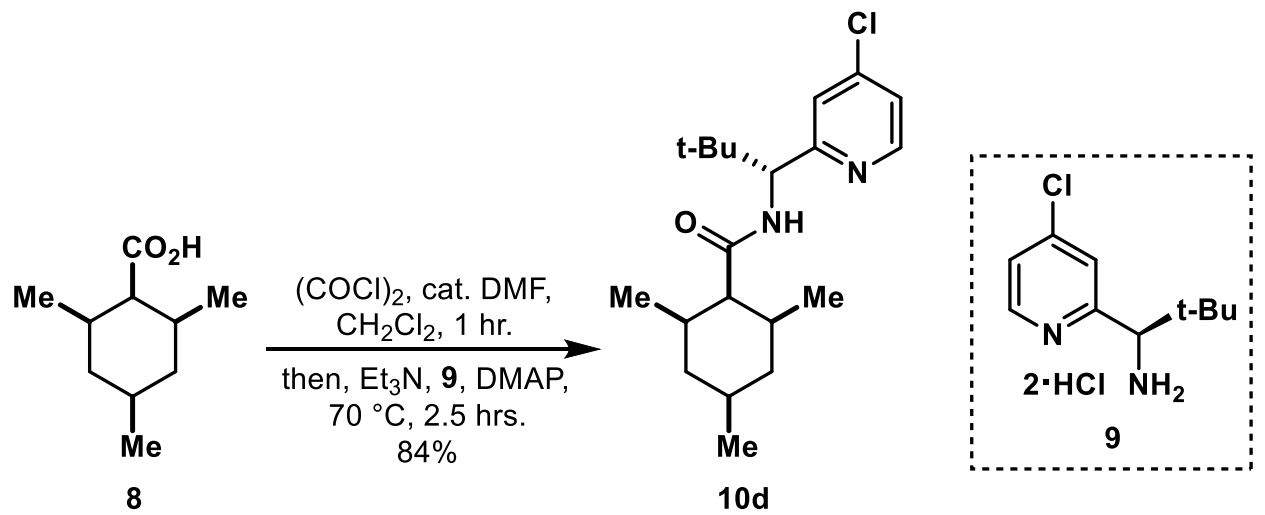

A flame dried round-bottomed flask was charged with 8 (6.7 g, $39.1 \mathrm{mmol}, 1.0$ equiv.) and $\mathrm{CH}_{2} \mathrm{Cl}_{2}$ (130 mL, $0.3 \mathrm{M})$. The flask was cooled to $0{ }^{\circ} \mathrm{C}$ and oxalyl chloride (4.0 mL, $46.9 \mathrm{mmol}, 1.2$ equiv.) was added, followed by a catalytic amount of DMF $(160 \mu \mathrm{L}, 2.0 \mathrm{mmol}, 0.05$ equiv.). The mixture was warmed to room temperature and allowed to stir until gas evolution ceased ( $c a .1 \mathrm{hr}$.). Solvent was removed under reduced pressure and the resulting yellow oil was re-suspended in toluene (20 $\mathrm{mL})$. In a separate flask, amine dihydrochloride 9 (10.6 g, $39.1 \mathrm{mmol}, 1.0$ equiv.) and DMAP (0.24 g, $2.0 \mathrm{mmol}, 0.05$ equiv.) were suspended in toluene $(70 \mathrm{~mL})$ and triethylamine $(19.5 \mathrm{~mL}, 145$ mmol, 3.7 equiv.) was added. This mixture was heated to $70{ }^{\circ} \mathrm{C}$ in an oil bath with vigorous 
stirring. The freshly prepared acid chloride was added dropwise via cannula transfer to the flask containing 9 , rinsing with an additional $15 \mathrm{~mL}$ of toluene. The heterogeneous brown mixture was allowed to stir at $70{ }^{\circ} \mathrm{C}$ for 2.5 hours. After cooling, the reaction was diluted with $\mathrm{CH}_{2} \mathrm{Cl}_{2}$ (300 $\mathrm{mL}$ ) and washed with saturated aqueous ammonium chloride $(400 \mathrm{~mL})$. The aqueous layer was back-extracted with $\mathrm{CH}_{2} \mathrm{Cl}_{2}(2 \mathrm{X} 125 \mathrm{~mL})$ and the combined organics were washed with brine $(250 \mathrm{~mL})$ and dried over anhydrous sodium sulfate. Filtration and concentration afforded a brown oil which was purified by column chromatography $(5 \rightarrow 10 \%$ EtOAc in hexanes) to afford the title compound 10d (11.6 g, $33.0 \mathrm{mmol}, 84 \%$ yield).

Physical state: white to off-white solid

TLC: $\mathrm{R}_{f}=0.3\left(10 \%\right.$ EtOAc in hexanes; visualization by $\left.\mathrm{UV}, \mathrm{KMnO}_{4}\right)$

m.p.: $72-74{ }^{\circ} \mathrm{C}$

$[\boldsymbol{\alpha}]_{\boldsymbol{D}}^{20.0}=+40.0\left(c=1.0, \mathrm{CHCl}_{3}\right)$

${ }^{1}$ H NMR (500 MHz, CDCl $): \delta 8.38(\mathrm{~d}, J=5.3 \mathrm{~Hz}, 1 \mathrm{H}), 7.17(\mathrm{~d}, J=1.7 \mathrm{~Hz}, 1 \mathrm{H}), 7.14(\mathrm{dd}, J=$ $5.3,1.9 \mathrm{~Hz}, 1 \mathrm{H}), 6.71(\mathrm{~d}, J=9.1 \mathrm{~Hz}, 1 \mathrm{H}), 4.83(\mathrm{~d}, J=9.3 \mathrm{~Hz}, 1 \mathrm{H}), 2.19(\mathrm{t}, J=4.7 \mathrm{~Hz}, 1 \mathrm{H}), 1.62$ $-1.71(\mathrm{~m}, 2 \mathrm{H}), 1.54(\mathrm{q}, J=12.2 \mathrm{~Hz}, 1 \mathrm{H}), 1.31-1.42(\mathrm{~m}, 3 \mathrm{H}), 1.22-1.25(\mathrm{~m}, 1 \mathrm{H}), 0.99(\mathrm{~d}, J=6.9$ $\mathrm{Hz}, 3 \mathrm{H}), 0.90$ (s, 9H), 0.87 (d, $J=6.2 \mathrm{~Hz}, 3 \mathrm{H}), 0.57$ (d, $J=6.9 \mathrm{~Hz}, 3 \mathrm{H})$.

${ }^{13} \mathbf{C}$ NMR (125 MHz, $\left.\mathbf{C D C l}_{3}\right): \delta$ 173.2, 161.0, 149.5, 143.6, 124.4, 122.6, 60.6, 53.3, 37.8, 37.7, 35.6, 35.2, 35.0, 32.6, 26.9, 22.5, 20.3, 19.8 .

HRMS (m/z): calculated for $\mathrm{C}_{20} \mathrm{H}_{32} \mathrm{ClN}_{2} \mathrm{O}[\mathrm{M}+\mathrm{H}]^{+}: 351.2198$, found 351.2200 .

Chiral SFC: The sample was analyzed on a Waters UPC2 SFC with a Daicel IG column $(3 \mu \mathrm{m}$, $4.6 \times 250 \mathrm{~mm}$ ) under isocratic conditions ( $4 \mathrm{~mL} / \mathrm{min}, 7 \% \mathrm{MeOH} / \mathrm{CO}_{2}, 1600$ psi backpressure) at $30{ }^{\circ} \mathrm{C}$. The enantiomers were detected by UV light $(260 \mathrm{~nm})$.

\section{Racemic-10d}

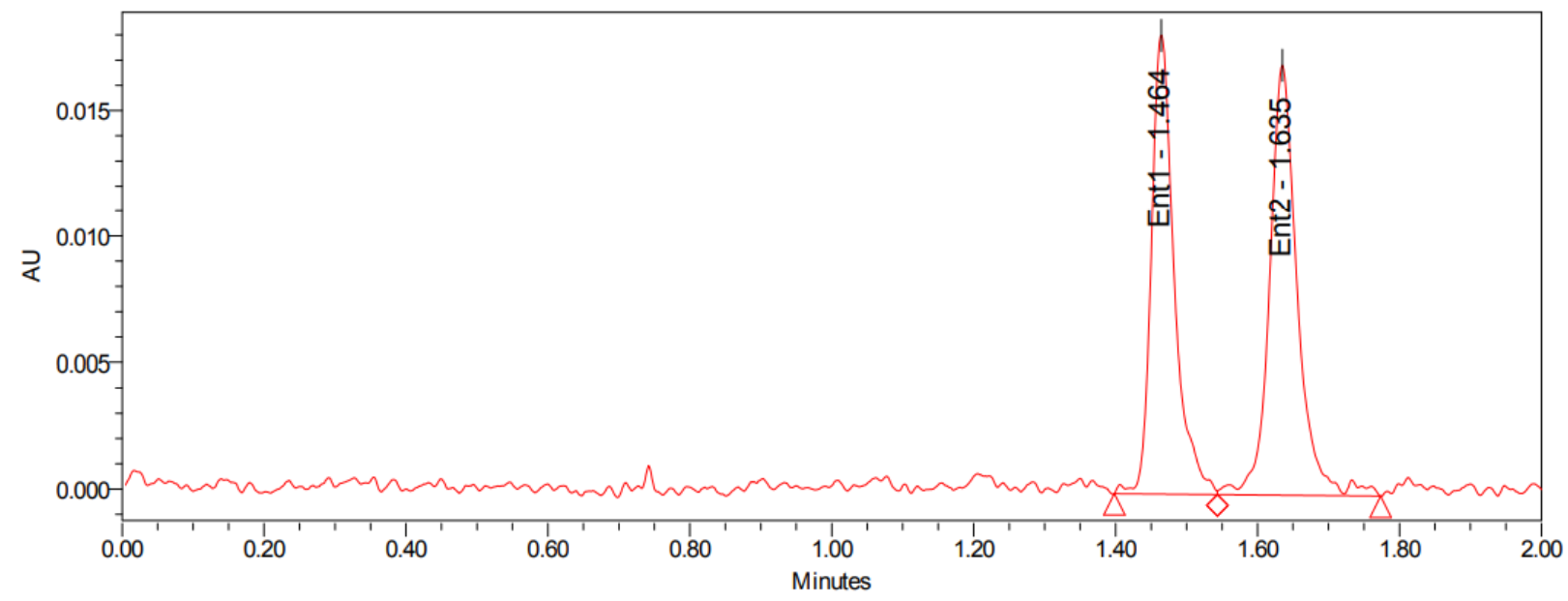




\section{Enantiopure-10d}

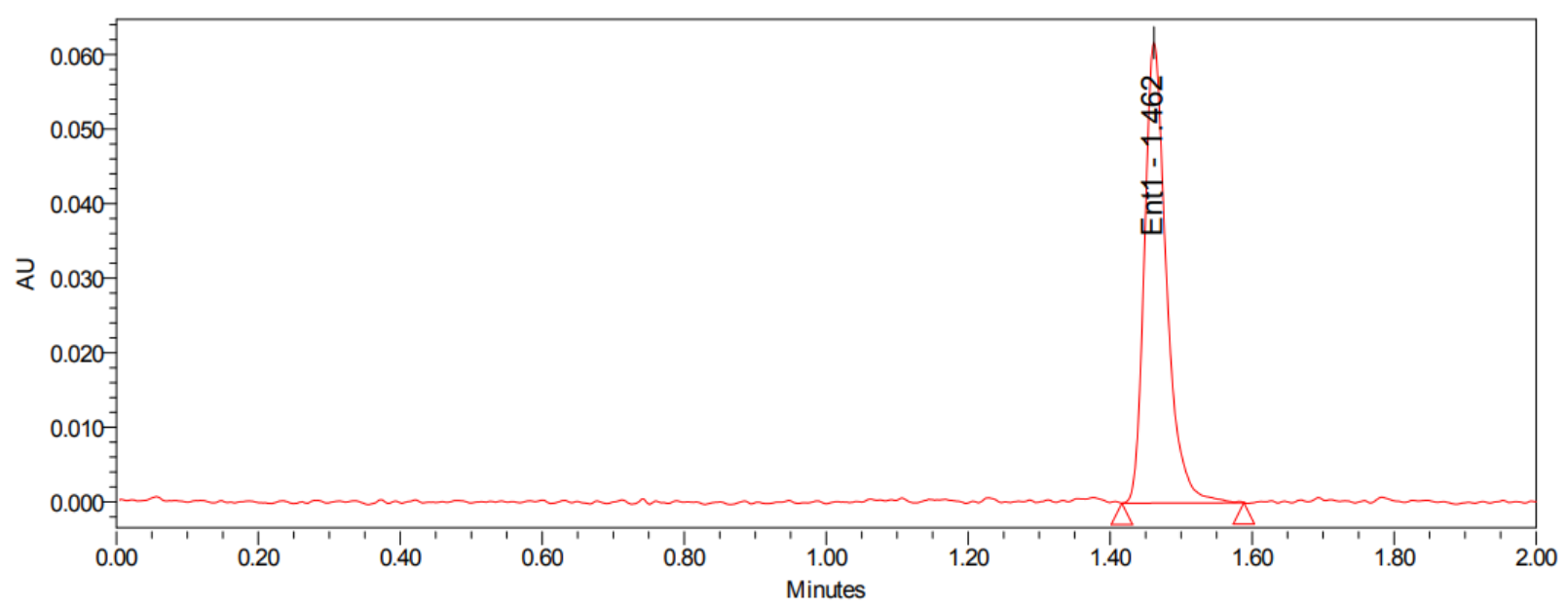

\section{Compound 11}

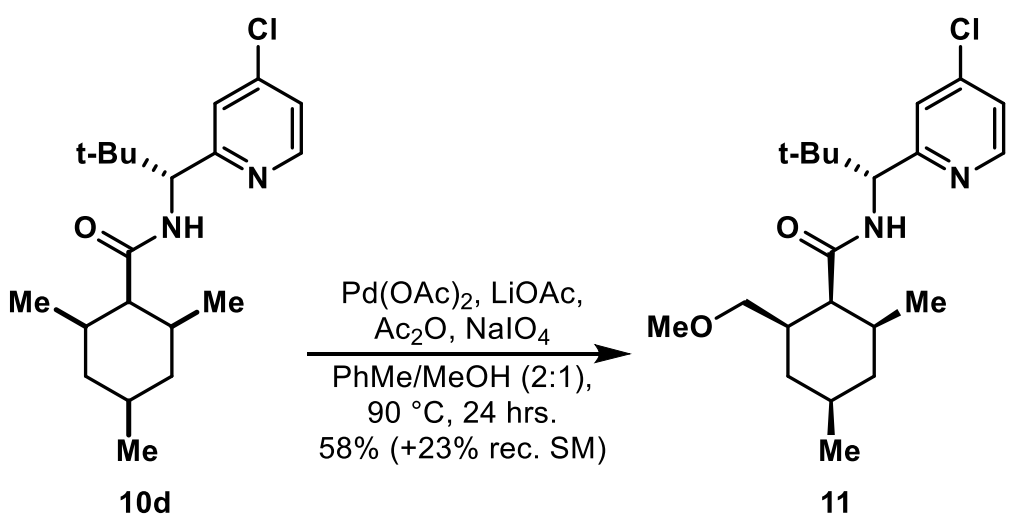

Two identical reactions were run in parallel. An oven dried $420 \mathrm{~mL}$ thick walled RBF (Note 1) equipped with stir bar was placed under argon atmosphere and charged with 10d $(3.00 \mathrm{~g}, 8.55$ mmol, 1.0 equiv.), $\mathrm{Pd}(\mathrm{OAc})_{2}$ ( $0.288 \mathrm{~g}, 1.28 \mathrm{mmol}, 0.15$ equiv.), anhydrous LiOAc $(0.564 \mathrm{~g}, 8.55$ mmol, 1.0 equiv.), and $\mathrm{NaIO}_{4}$ (7.32 g, $34.2 \mathrm{mmol}, 4.0$ equiv.) (Note 2). The flask was evacuated and backfilled with argon three times before a 2:1 mixture of toluene/methanol $(170 \mathrm{~mL}, 0.05 \mathrm{M})$ ) was added (Note 3). Acetic anhydride $(1.6 \mathrm{~mL}, 17.1 \mathrm{mmol}, 2.0$ equiv.) was added via syringe and the flask was quickly sealed using a PTFE screw cap. The orange reaction mixture was transferred to an oil bath preheated to $90{ }^{\circ} \mathrm{C}$ and allowed to stir at this temperature for $24 \mathrm{hrs}$ behind a blast shield (Note 4). Upon completion, the reaction was allowed to cool to room temperature, then diluted with EtOAc $(150 \mathrm{~mL})$ and filtered over Celite ${ }^{\circledR}$. The filtrate from both reactions was combined and partially concentrated under reduced pressure to remove residual methanol. The heterogeneous orange residue was re-suspended in EtOAc $(300 \mathrm{~mL})$ and transferred to a separatory funnel. The organic layer was washed with saturated aqueous ammonium chloride solution (200 $\mathrm{mL})$ and the aqueous layer was back-extracted with EtOAc $(150 \mathrm{~mL})$. The combined organics were washed with water $(200 \mathrm{~mL})$ and brine $(200 \mathrm{~mL})$, then dried with sodium sulfate, filtered and 
concentrated in-vacuo. The resulting oil was purified by flash chromatography $(10 \rightarrow 20 \%$ EtOAc in hexanes) to provide recovered starting material $10 \mathrm{~d}(1.39 \mathrm{~g}, 3.96 \mathrm{mmol})$ and the desymmetrized product 11 as an orange oil (3.78 g, $9.92 \mathrm{mmol}, 58 \%$ yield, $75 \% \mathrm{BRSM})$.

Note 1 - Flask purchased from Chemglass (CG-1880-R-04)

Note 2 - An adapter with 24/40 ground glass joint and septum was used during this process and the flask was stoppered between solid additions.

Note 3 - Anhydrous, degassed solvent was used for this reaction, the introduction of moisture or oxygen was found to promote early catalyst deactivation.

Note 4 - The reaction was stirred at $700 \mathrm{rpm}$. After reaching $90{ }^{\circ} \mathrm{C}$ the mixture transitions from orange to bright yellow and remains this color for the course of the reaction. Significant pressure is generated and a thick walled, sealed tube with adequate head space (approx. half the flask volume) should be used.

Physical state: yellow oil

TLC: : $\mathrm{R}_{f}=0.3\left(15 \%\right.$ EtOAc in hexanes; visualization by $\left.\mathrm{UV}, \mathrm{KMnO}_{4}\right)$

$[\boldsymbol{\alpha}]_{\boldsymbol{D}}^{\mathbf{2 0 . 0}}=+39.4\left(c=1.0, \mathrm{CHCl}_{3}\right)$

${ }^{1}$ H NMR (400 MHz, CDCl $): \delta 8.38(\mathrm{~d}, J=5.3 \mathrm{~Hz}, 1 \mathrm{H}), 7.15(\mathrm{~d}, J=1.8 \mathrm{~Hz}, 1 \mathrm{H}), 7.13(\mathrm{dd}, J=$ $5.3,2.0 \mathrm{~Hz}, 1 \mathrm{H}), 6.81(\mathrm{~d}, J=9.1 \mathrm{~Hz}, 1 \mathrm{H}), 4.80(\mathrm{~d}, J=9.2 \mathrm{~Hz}, 1 \mathrm{H}), 3.32(\mathrm{~s}, 3 \mathrm{H}), 3.31(\mathrm{dd}, J=9.3$, $9.9 \mathrm{~Hz}, 1 \mathrm{H}), 3.23(\mathrm{dd}, J=9.2,5.4 \mathrm{~Hz}, 1 \mathrm{H}), 2.47(\mathrm{t}, J=4.6 \mathrm{~Hz}, 1 \mathrm{H}), 1.93-1.85(\mathrm{~m}, 1 \mathrm{H}), 1.63-$ $1.51(\mathrm{~m}, 1 \mathrm{H}), 1.51-1.35(\mathrm{~m}, 3 \mathrm{H}), 1.30-1.20(\mathrm{~m}, 2 \mathrm{H}), 0.90(\mathrm{~s}, 9 \mathrm{H}), 0.87(\mathrm{~d}, J=5.6 \mathrm{~Hz}, 3 \mathrm{H}), 0.57$ $(\mathrm{d}, J=6.9 \mathrm{~Hz}, 3 \mathrm{H})$.

${ }^{13}$ C NMR (100 MHz, $\left.\mathbf{C D C l}_{3}\right): \delta 172.8,161.0,149.5,143.6,124.2,122.5,76.0,60.8,58.8,47.9$, $40.8,38.1,35.6,34.8,32.4,32.2,26.8,22.4,19.7$.

HRMS (m/z): calculated for $\mathrm{C}_{21} \mathrm{H}_{34} \mathrm{ClN}_{2} \mathrm{O}_{2}[\mathrm{M}+\mathrm{H}]^{+}: 381.2303$, found 381.2300 .
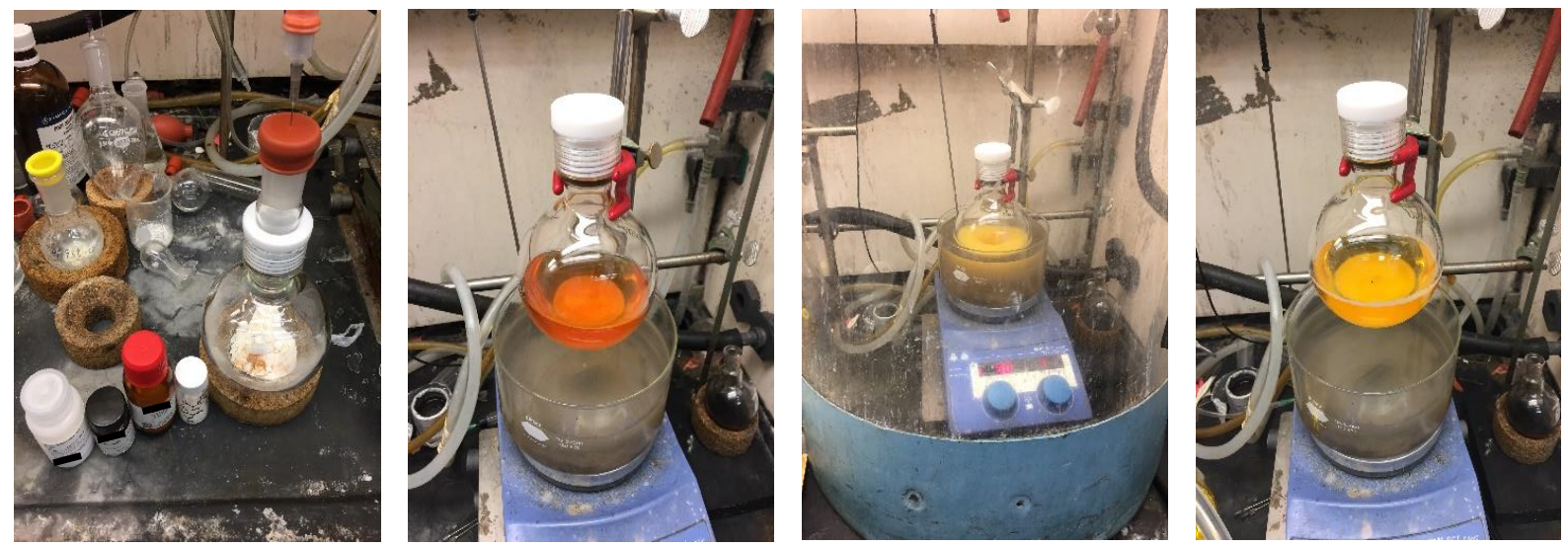

(left) solid reagents added to flask; (middle-left) reaction appearance prior to heating; (middleright) reaction after $c a .10$ minutes at $90{ }^{\circ} \mathrm{C}$; (right) reaction after $24 \mathrm{hrs}$. 


\section{Compound 12}

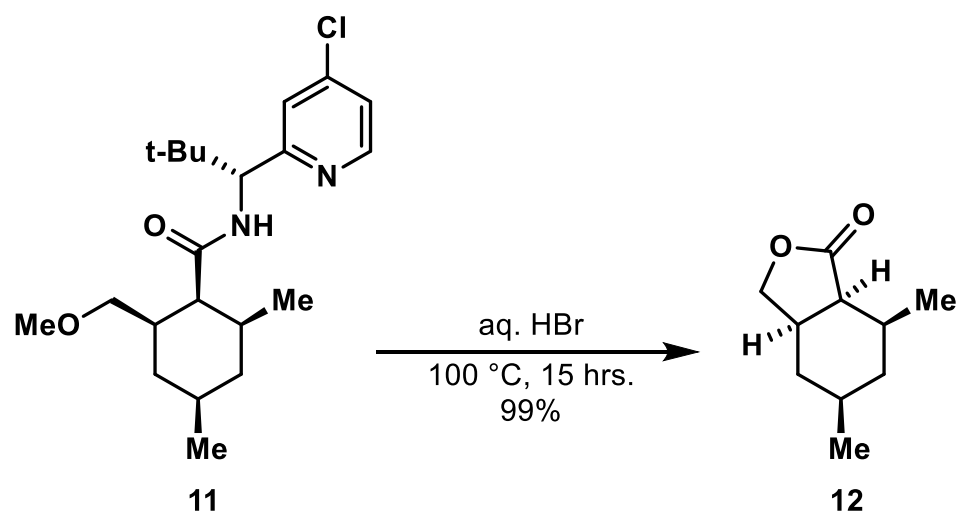

Compound 11 (3.49 g, $9.18 \mathrm{mmol}, 1.00$ equiv.) was transferred to a $350 \mathrm{~mL}$ sealed tube and a stir bar was added. The residue was suspended in $48 \%$ aqueous hydrobromic acid $(60 \mathrm{~mL}, 0.15 \mathrm{M})$ to create a yellow suspension (Note 1). Water was added $(60 \mathrm{~mL}, 0.15 \mathrm{M})$ and the mixture was sealed and transferred in an oil bath pre-heated to $100{ }^{\circ} \mathrm{C}$. The mixture was stirred vigorously (1100 rpm) for $15 \mathrm{hrs}$ (Note 2). After cooling, the mixture was diluted with $\mathrm{Et}_{2} \mathrm{O}(200 \mathrm{~mL})$ and transferred to a separatory funnel containing water $(60 \mathrm{~mL})$. The layers were separated, and the aqueous layer was extracted with an additional $100 \mathrm{~mL}$ of $\mathrm{Et}_{2} \mathrm{O}$. The combined organics were washed with brine, dried with sodium sulfate, filtered and concentrated in-vacuo. The resulting brown oil was passed through a plug of silica gel $\left(40 \% \mathrm{Et}_{2} \mathrm{O}\right.$ in hexanes) and concentrated to afford 12 (1.53 g, 9.09 mmol, $99 \%$ yield).

The initial aqueous layer was cooled to $0{ }^{\circ} \mathrm{C}$ and basified using $3 \mathrm{~N} \mathrm{NaOH}$. The cloudy suspension was extracted with EtOAc ( 3 X $120 \mathrm{~mL})$ and the combined organics were washed with brine (100 $\mathrm{mL})$, dried with sodium sulfate and concentrated in-vacuo to afford the free-base of 9 (1.46 g, 7.34 mmol, $80 \%$ recovery) as an orange oil (Note 3 ).

Note 1 - Sonicating the starting material significantly accelerates the dissolution process. It is important to ensure that starting material it is completely suspended in the $\mathrm{HBr}$ solution prior to initiating the reaction as it can form an insoluble residue on the stir bar/reaction vessel.

Note 2 - Over the course of the reaction the product phases out and can be observed as a brown oil on the surface of the aqueous reaction solvent. Reaction monitored by LCMS.

Note 3 - The recovered directing group also contained $0.218 \mathrm{~g}(0.90 \mathrm{mmol})$ of the 4-bromo derivative which was carried through the DG installation $>\mathrm{C}-\mathrm{H}$ activation $>$ deprotection recycling sequence without issue.

Physical state: yellow oily solid

TLC: $\mathrm{R}_{f}=0.25\left(20 \%\right.$ EtOAc in hexanes; visualization by $\left.\mathrm{KMnO}_{4}\right)$

$[\alpha]_{D}^{20.0}=+6.7\left(c=1.0, \mathrm{CHCl}_{3}\right)$

${ }^{1}$ H NMR (600 MHz, CDCl3): $\delta 4.16(\mathrm{dd}, J=8.7,4.5 \mathrm{~Hz}, 1 \mathrm{H}), 3.89(\mathrm{~d}, J=8.7 \mathrm{~Hz}, 1 \mathrm{H}), 2.56(\mathrm{t}, J$ $=5.7 \mathrm{~Hz}, 1 \mathrm{H}), 2.41(\mathrm{ddd}, J=12.1,10.5,5.9 \mathrm{~Hz}, 1 \mathrm{H}), 1.93-1.81(\mathrm{~m}, 1 \mathrm{H}), 1.75(\mathrm{dt}, J=13.5,2.8$ 
$\mathrm{Hz}, 1 \mathrm{H}), 1.59-1.51(\mathrm{~m}, 1 \mathrm{H}), 1.46-1.38(\mathrm{~m}, 1 \mathrm{H}), 1.32(\mathrm{~d}, J=7.2 \mathrm{~Hz}, 3 \mathrm{H}), 0.94-0.81(\mathrm{~m}, 2 \mathrm{H})$, $0.91(\mathrm{~d}, J=6.6 \mathrm{~Hz}, 3 \mathrm{H})$.

${ }^{13}$ C NMR (150 MHz, CDCl $)$ : $\delta$ 176.7, 71.5, 44.4, 39.4, 37.7, 35.9, 31.5, 31.3, 22.4, 18.3 .

HRMS (m/z): calculated for $\mathrm{C}_{10} \mathrm{H}_{17} \mathrm{O}_{2}[\mathrm{M}+\mathrm{H}]^{+}:$169.1223, found 169.1222.

Chiral GCMS: An Agilent Technologies 7890A gas chromatography (USA) equipped with a CPChirasil-DEX CB column ( $25 \mathrm{~m} \times 0.25 \mathrm{~mm} \times 0.25 \mu \mathrm{m})$ and a flame ionization detector (FID) was employed for the chiral separation and quantification of the enantiomeric mixture. Helium $(99.999 \%)$ was used as the carrier gas, with a constant flow rate of $1.0 \mathrm{~mL} / \mathrm{min}$. The optimized temperature program applied in the GC-FID was started from $125^{\circ} \mathrm{C}$ and raised to $200^{\circ} \mathrm{C}$ at the rate of $3{ }^{\circ} \mathrm{C} / \mathrm{min}$. The temperature of the detector and injector were held at $200^{\circ} \mathrm{C}$ and $300^{\circ} \mathrm{C}$ respectively. The inlet split ratio was 50:1.

\section{Racemic-12}

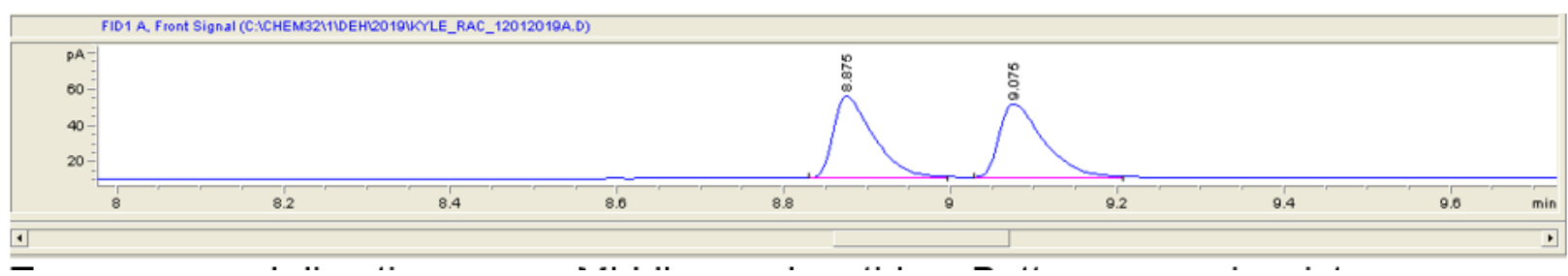

12

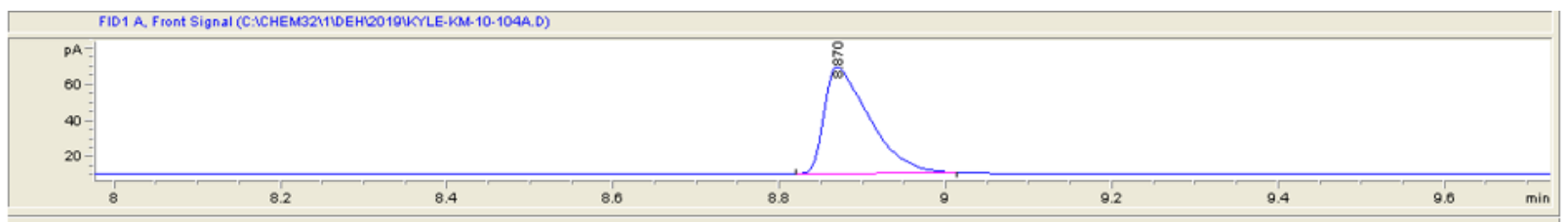

12 (from 11 using recycled directing group)

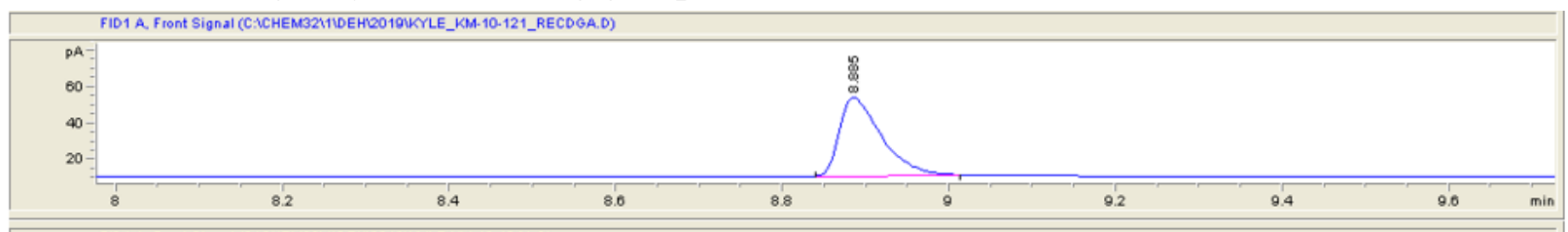



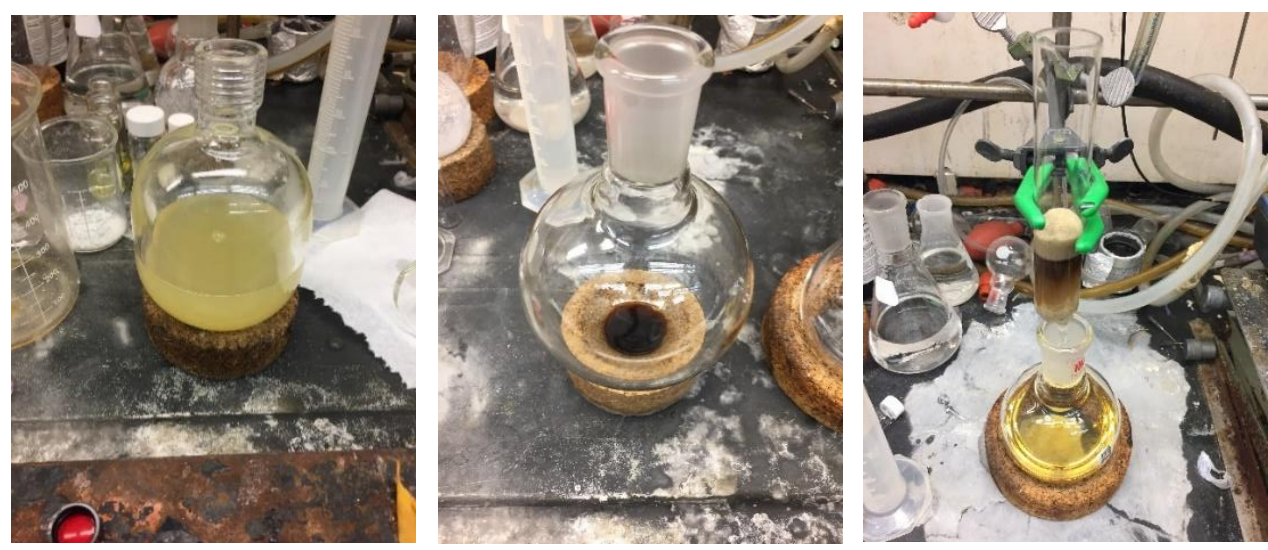

(left) starting material suspended in aq. $\mathrm{HBr}$ prior to heating; (middle) crude product after extractive w/u; (right) purification through plug of silica gel.

\section{Racemic Synthesis 12}

\section{Compound SI-11}

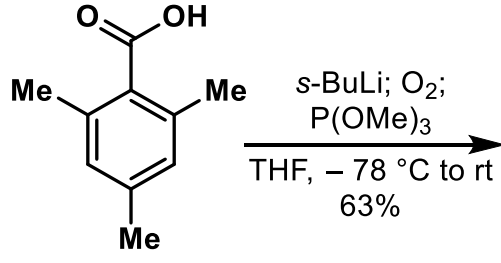<smiles>Cc1cc(C)c2c(c1)COC2=O</smiles>

SI-11

Initial sequence used oxygen as the oxidizing agent. Although this reaction was scalable, it proved hazardous, and resulted in an explosion on one occasion. An alternative, safer procedure was developed which also resulted in a more efficient reaction:

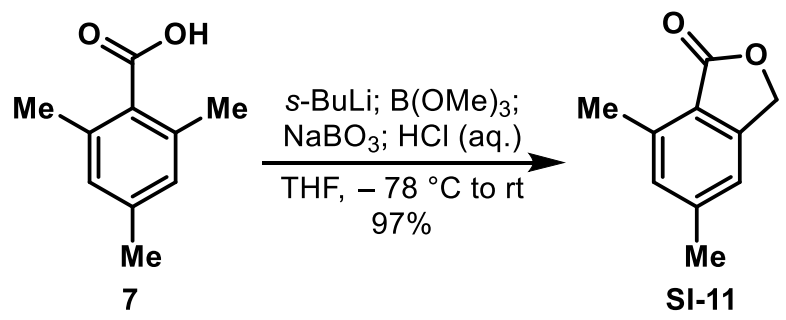

An oven dried $2 \mathrm{~L}$ 3-neck round bottomed flask was fitted with a mechanical stirrer and purged with argon. Acid 7 (10.8 g, $66.0 \mathrm{mmol}, 1.0$ equiv.) and anhydrous THF (330 mL, $0.2 \mathrm{M}$ ) were added and the mixture was cooled to $-78{ }^{\circ} \mathrm{C}$. A $1.35 \mathrm{M}$ solution of sec-Butyl lithium in cyclohexane (9.4 mL, $12.8 \mathrm{mmol}, 2.1$ equiv.) was slowly added over 10 mins with stirring. (Note 1) The resulting deep red solution was allowed to stir at this temperature for 2 hours before a $1 \mathrm{M}$ THF solution of $\mathrm{B}(\mathrm{OMe})_{3}(9.3 \mathrm{~mL}, 83.0 \mathrm{mmol}, 1.25$ equiv.) was added, down the side of the reaction flask. (Note 2) The resulting mixture was warmed to $0{ }^{\circ} \mathrm{C}$ using an ice bath and allowed to stir for 30 mins before slowly quenching with $330 \mathrm{~mL}$ water (caution, gas evolution). 
$\mathrm{NaBO}_{3} \times 4 \mathrm{H}_{2} \mathrm{O}$ (41 g, $264 \mathrm{mmol}, 4.0$ equiv.) was added in $10 \mathrm{~g}$ portions and the heterogeneous mixture was stirred vigorously at ambient temperature overnight. A solution of $2 \mathrm{~N} \mathrm{HCl}(330 \mathrm{~mL})$ was then added and the biphasic mixture was stirred vigorously for 1 hour before transferring to a separatory funnel with $\mathrm{CH}_{2} \mathrm{Cl}_{2}(500 \mathrm{~mL})$. Saturated aqueous sodium bicarbonate was added until a basic $\mathrm{pH}$ was reached (CAUTION, SIGNIFICANT GAS EVOLUTION). The layers were separated, and the aqueous phase was extracted with an additional $300 \mathrm{~mL}$ of $\mathrm{CH}_{2} \mathrm{Cl}_{2}$. The combined organic extracts were washed with half-saturated brine, dried with sodium sulfate, filtered and concentrated in-vacuo to provide the title compound SI-11 as white crystals (10.4 g, $64.2 \mathrm{mmol}, 97 \%$ yield). The product was pure by ${ }^{1} \mathrm{H}$ NMR and could be used directly in the next step.

Note $1-s$-BuLi was added via cannula transfer from a commercial bottle (Sigma).

Note 2 - Efficient stirring is crucial for this step (mechanical stirrer recommended for large scale). The reaction becomes quite thick during the addition of $\mathrm{B}(\mathrm{OMe})_{3}$ and if stirring is stopped a dimerization by-product results.

${ }^{1} \mathbf{H}$ NMR (600 MHz, CDCl $): \delta 7.07$ (d, $\left.J=8.3 \mathrm{~Hz}, 2 \mathrm{H}\right), 5.19$ (s, 2H), 2.64 (s, 3H), 2.43 (s, 3H).

Spectral data matches the literature. ${ }^{11}$
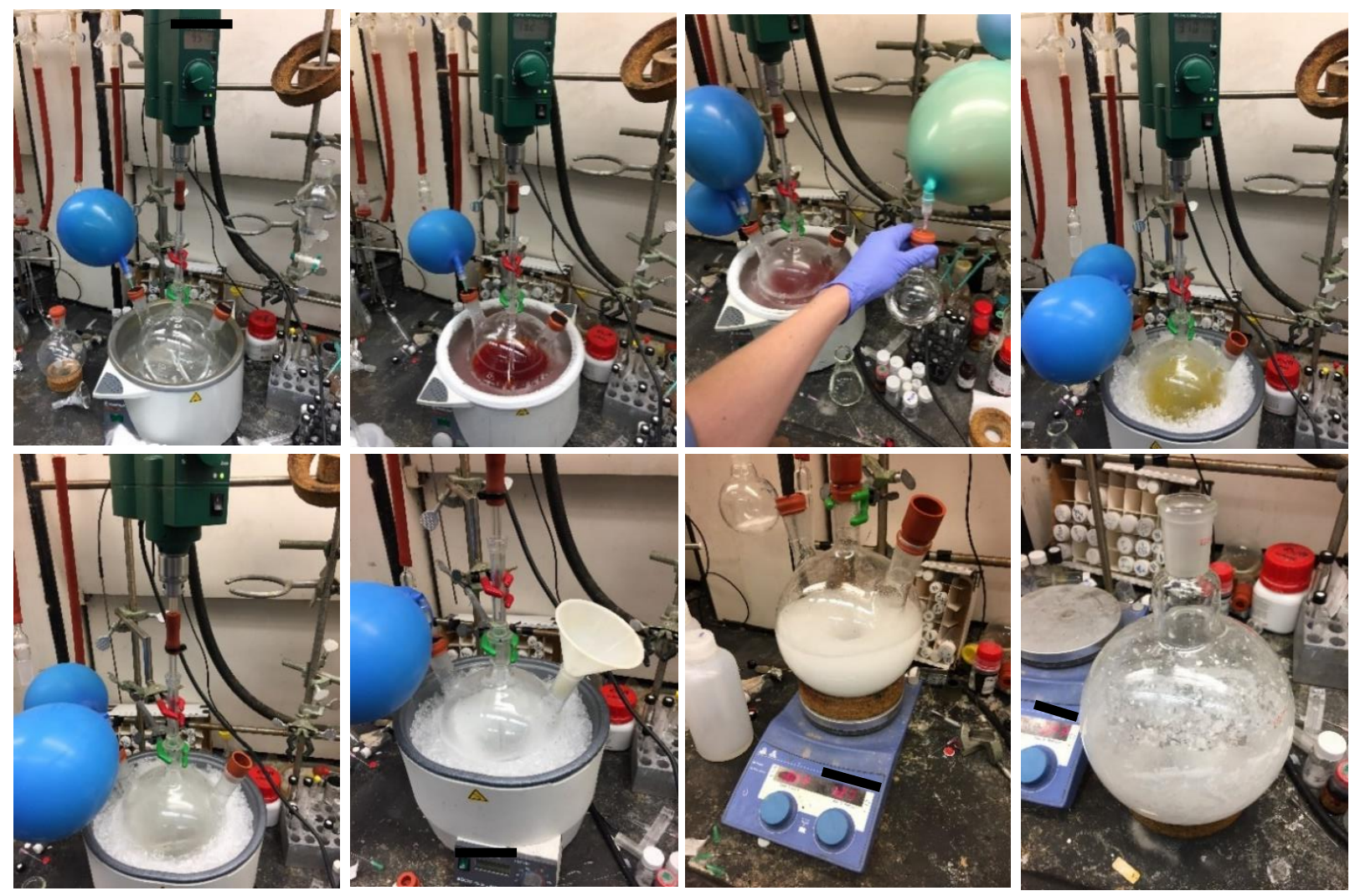

(top-left) SM dissolved in THF, (top-middle-left) after addition of $s$-BuLi, (top-middle-right) addition of $\mathrm{B}(\mathrm{OMe})_{3}$ in $\mathrm{THF}$, (top-right) immediately after transferring to ice bath, (bottom-left) after 30 mins at $0{ }^{\circ} \mathrm{C}$, (bottom-middle-left) addition of $\mathrm{NaBO}_{3}$, (bottom-middle-right) after addition of aq. $\mathrm{HCl}$, (bottom-right) pure product after extraction and concentration. 


\section{Compound rac-12}

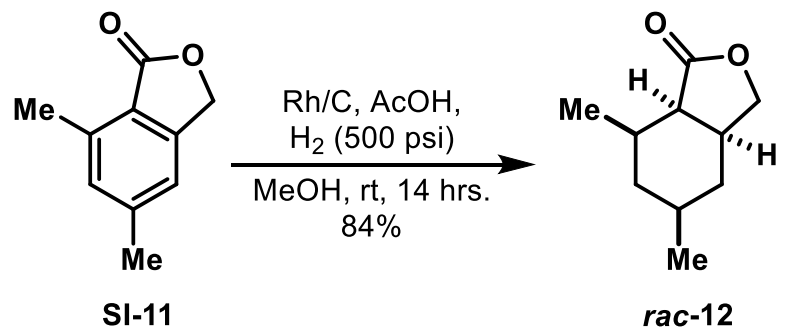

A $100 \mathrm{~mL}$ round bottomed flask equipped with stir bar was charged with lactone SI-11 (5.17 g, $31.9 \mathrm{mmol}, 1.00$ equiv.) and rhodium on carbon (4.00 g, ca. $6 \mathrm{~mol} \% \mathrm{Rh}$ ) (Note 1). The flask was evacuated, backfilled with argon and sealed with a septum. Degassed methanol (40 mL, $0.8 \mathrm{M})$ (Note 2 ) was added under argon, followed by glacial acetic acid $(0.5 \mathrm{~mL}, 1.25 \% / \mathrm{V})$. The flask was transferred to a Parr pressure vessel (Note 3) and a blanket of argon was blown over the apparatus before the septum was removed from the flask and the Parr apparatus sealed. The pressure vessel was pressurized with hydrogen (400 psi) and vented, this process was repeated twice. (Note 4) The vessel was then pressurized to 500 psi with hydrogen and transferred to a stir plate. The reaction was allowed to stir (behind a blast shield) at $1400 \mathrm{rpm}$ for $14 \mathrm{hrs}$. After venting, the flask was removed from the Parr vessel and the flask contents were filtered over Celite ${ }^{\circledR}$, rinsing with methanol. (Note 5) The clear filtrate was concentrated under reduced pressure and subjected to column chromatography (30\% $\mathrm{Et}_{2} \mathrm{O}$ in hexanes) to afford rac-12 (4.49 g, 26.7 mmol, 84\% yield).

Note 1 - Rhodium on carbon 5\%/wt loading, 50\%/wt water, obtained from Johnson-Matthey.

Note 2 - Methanol was degassed by sparging with argon for 15 mins. Using non-degassed methanol or adding solvent under air atmosphere can cause the methanol to ignite.

Note 3 - The flask was supported by a rubber filter adapter to keep it upright inside the vessel.

Note 4 - Pressurization/venting conducted behind a blast shield. This step is essential for reaction success.

Note 5 - Use extreme caution when filtering reaction mixture. Methanol can ignite in the presence of rhodium catalyst. Never let Celite ${ }^{\circledR}$ filtration bed run completely dry. Treat spent metal catalyst with excess water and dispose of appropriately.

For characterization data, see synthesis of $\mathbf{1 2}$.

\section{Compound 14}

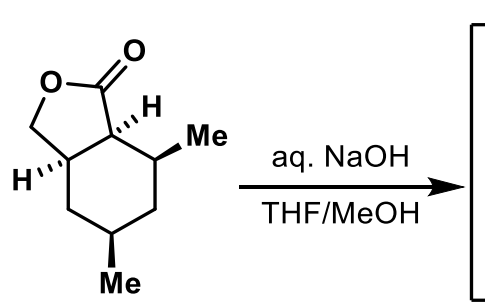

12

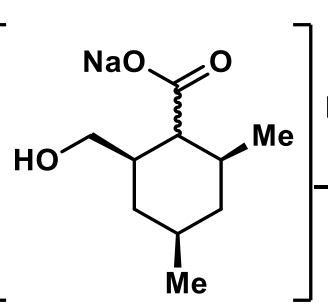

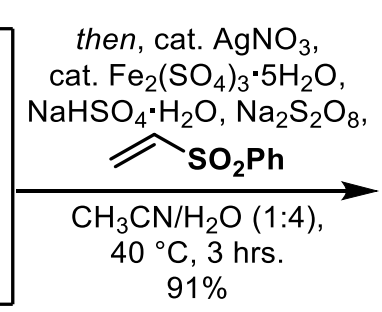

$91 \%$

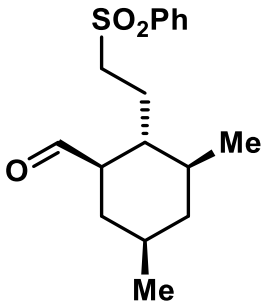

14 
In a $250 \mathrm{~mL}$ RBF, lactone 12 (1.507 g, $8.97 \mathrm{mmol}, 1.00$ equiv.) was suspended in THF (22 mL, $0.41 \mathrm{M})$ and an aqueous solution of sodium hydroxide $(0.430 \mathrm{~g}, 10.76 \mathrm{mmol}, 1.20$ equiv. $)$ in water $(5.6 \mathrm{~mL}, 1.6 \mathrm{M})$ was added with stirring. Methanol $(2.6 \mathrm{~mL}, 3.4 \mathrm{M})$ was added and the reaction was stirred vigorously at room temperature overnight (Note 1). Solvent was removed in-vacuo and the resulting oily residue was co-evaporated twice with benzene to afford an off-white solid. To this was added $\mathrm{AgNO}_{3}$ (0.457 g, $2.69 \mathrm{mmol}, 0.30$ equiv.), $\mathrm{Fe}_{2}\left(\mathrm{SO}_{4}\right)_{3} \cdot 5 \mathrm{H}_{2} \mathrm{O}(0.877 \mathrm{~g}, 1.79 \mathrm{mmol}$, 0.20 equiv.), $\mathrm{NaHSO}_{4} \bullet \mathrm{H}_{2} \mathrm{O}$ (1.40 g, $10.14 \mathrm{mmol}, 1.13$ equiv.), $\mathrm{Na}_{2} \mathrm{~S}_{2} \mathrm{O}_{8}$ (5.338 g, $22.43 \mathrm{mmol}$, 2.50 equiv.) and phenyl vinyl sulfone ( $2.110 \mathrm{~g}, 12.56 \mathrm{mmol}, 1.40$ equiv.). The flask was evacuated and backfilled with argon three times then sealed with a rubber septum and charged with a 4:1 solution of degassed water/ $\mathrm{CH}_{3} \mathrm{CN}(90 \mathrm{~mL}, 0.1 \mathrm{M})$ (Note 2). The mixture was transferred to an oil bath pre-heated to $40^{\circ} \mathrm{C}$ and stirred vigorously (1050 rpm) for 3 hours (Note 3 ). Upon completion, the reaction was diluted with $\mathrm{Et}_{2} \mathrm{O}(150 \mathrm{~mL})$ and transferred to a separatory funnel containing 150 $\mathrm{mL}$ aqueous tetrasodium EDTA (Note 4). The layers were separated and the aqueous layer was back-extracted with $\mathrm{Et}_{2} \mathrm{O}(150 \mathrm{~mL})$, the combined organics were washed with brine $(100 \mathrm{~mL})$, dried with sodium sulfate, filtered and concentrated under reduced pressure. The crude aldehyde 14 was obtained as a yellow oil (2.51 g, $8.15 \mathrm{mmol}, 91 \%$ yield) and was sufficiently pure to be used directly in the next step (Note 5). A small quantity was purified by column chromatography $\left(50 \% \mathrm{Et}_{2} \mathrm{O}\right.$ in hexanes) for characterization.

Note 1 - The reaction is initially cloudy but becomes clear after overnight stirring.

Note 2 - A pre-mixed 4:1 solution of water to acetonitrile was sparged with argon using a 20gauge needle under sonication for 20 minutes.

Note 3 - A high stir rate is important to prevent clumping. It is recommended that the reaction be fitted with an argon balloon on larger scale $(>1 \mathrm{mmol})$ as the generation of $\mathrm{CO}_{2}$ can create significant back-pressure.

Note 4 - EDTA solution prepared by mixing $30 \mathrm{~mL}$ saturated aqueous Na4EDTA with $120 \mathrm{~mL}$ deionized water.

Note 5 - Yield calculated based on crude NMR, the product decomposes on silica gel.

Physical state: colorless oil

TLC: $\mathrm{R}_{f}=0.3\left(50 \% \mathrm{Et}_{2} \mathrm{O}\right.$ in hexanes; visualization by $\left.\mathrm{UV}, \mathrm{KMnO}_{4}\right)$

$[\boldsymbol{\alpha}]_{\boldsymbol{D}}^{\mathbf{2 0 . 0}}=+15.2\left(c=1.0, \mathrm{CHCl}_{3}\right)$

${ }^{1}$ H NMR (600 MHz, CDCl3): $\delta 9.38(\mathrm{~d}, J=3.6 \mathrm{~Hz}, 1 \mathrm{H}), 7.88(\mathrm{dd}, J=8.3,1.1 \mathrm{~Hz}, 2 \mathrm{H}), 7.65(\mathrm{t}, J$ $=7.5 \mathrm{~Hz}, 1 \mathrm{H}), 7.56(\mathrm{t}, J=7.8 \mathrm{~Hz}, 2 \mathrm{H}), 3.06(\mathrm{tt}, J=9.1,8.0 \mathrm{~Hz}, 2 \mathrm{H}), 2.11(\mathrm{tt}, J=12.1,3.7 \mathrm{~Hz}$, $1 \mathrm{H}), 1.90-1.84(\mathrm{~m}, 1 \mathrm{H}), 1.75(\mathrm{dq}, J=12.8,3.5 \mathrm{~Hz}, 1 \mathrm{H}), 1.70-1.63(\mathrm{~m}, 2 \mathrm{H}), 1.48-1.40(\mathrm{~m}$, $1 \mathrm{H}), 1.34(\mathrm{tt}, J=11.1,3.8 \mathrm{~Hz}, 1 \mathrm{H}), 1.26-1.19(\mathrm{~m}, 1 \mathrm{H}), 0.89(\mathrm{~d}, J=6.6 \mathrm{~Hz}, 3 \mathrm{H}), 0.91-0.87(\mathrm{~m}$, $1 \mathrm{H}), 0.85(\mathrm{~d}, J=6.5 \mathrm{~Hz}, 3 \mathrm{H}), 0.73-0.67(\mathrm{~m}, 1 \mathrm{H})$.

${ }^{13}$ C NMR (150 MHz, CDCl 3$): \delta 203.5,138.8,133.9,129.4,128.2$, 54.0, 52.3, 43.7, 40.8, 34.9, $34.2,31.3,23.2,22.3,19.6$. 
HRMS (m/z): calculated for $\mathrm{C}_{17} \mathrm{H}_{25} \mathrm{O}_{3} \mathrm{~S}[\mathrm{M}+\mathrm{H}]^{+}: 309.1519$, found 309.1520 .

\section{Crude NMR}
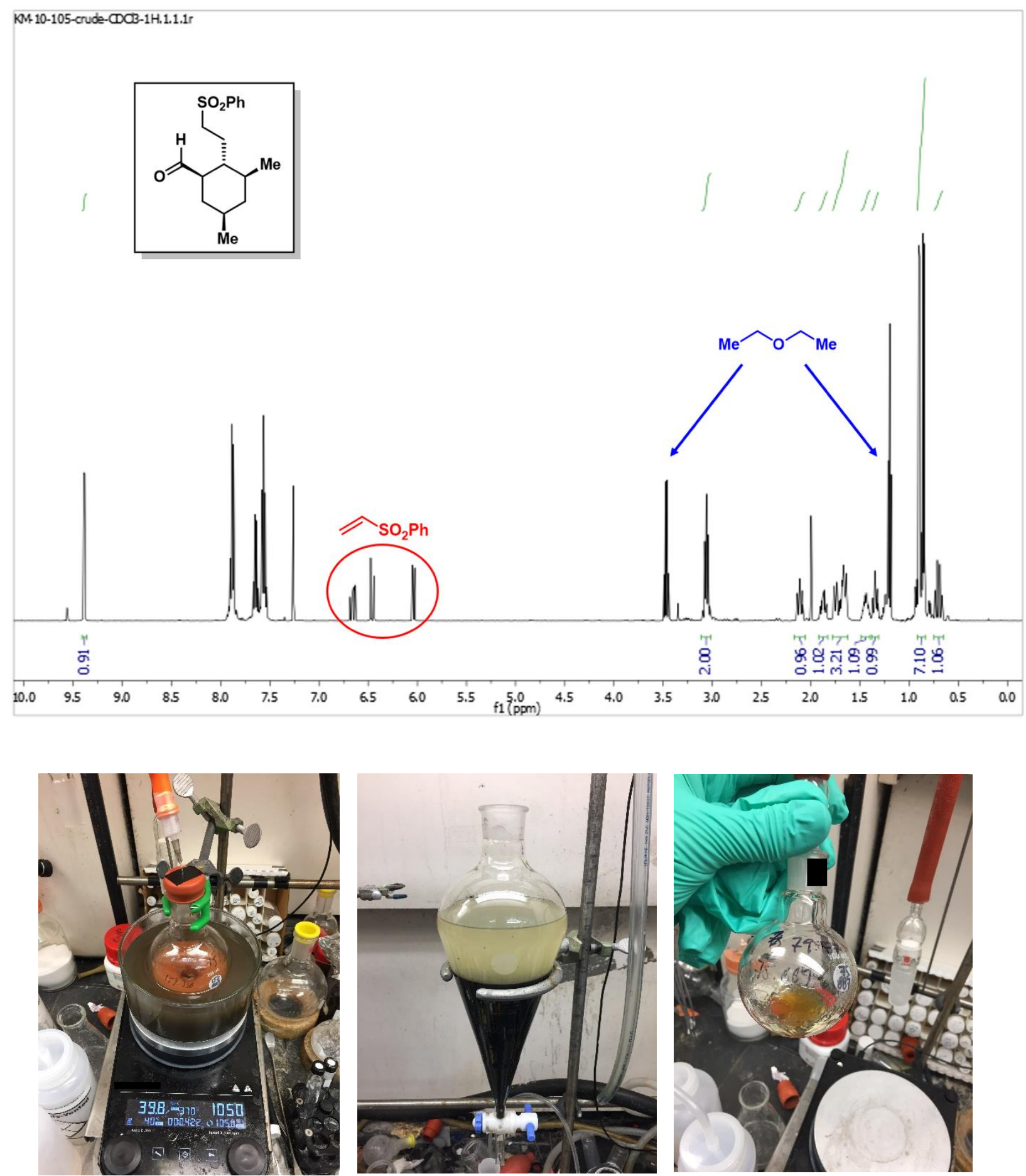

(left) reaction mixture ( $t=5$ mins); (middle) extraction with aq. EDTA; (right) crude product. 


\section{Compound SI-12}

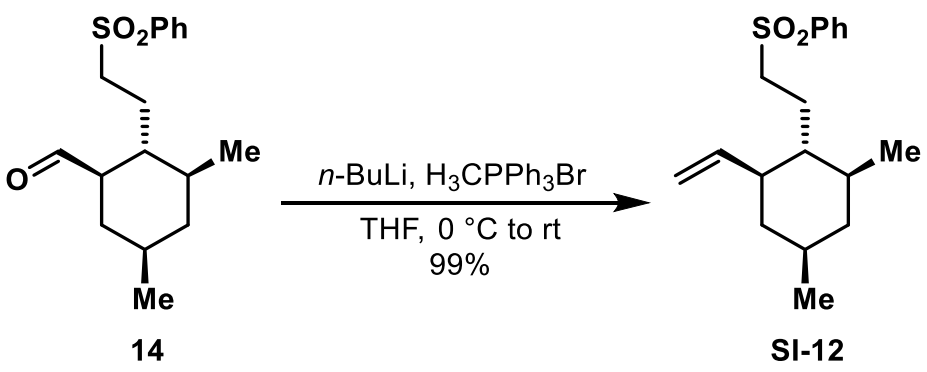

A $250 \mathrm{~mL}$ RBF equipped with stir bar was charged with methyltriphenylphosphonium bromide (7.20 g, $20.2 \mathrm{mmol}, 2.5$ equiv.) and dried under high vacuum with the aid of a heat gun (Note 1). After cooling, the flask was backfilled with argon and sealed with a rubber septum. The flask contents were suspended in anhydrous THF $(50 \mathrm{~mL})$ and cooled to $-5^{\circ} \mathrm{C}$ (Note 2) before $n$-butyl lithium (2.5 $\mathrm{M}$ in hexane) $(7.4 \mathrm{~mL}, 18.6 \mathrm{mmol}, 2.3$ equiv.) was added dropwise with vigorous stirring. The yellow mixture was allowed to stir 30 minutes before aldehyde $14(2.49 \mathrm{~g}, 8.07 \mathrm{mmol}$, 1.0 equiv.) in THF $(25 \mathrm{~mL})$ was added dropwise via cannula transfer, chased with a $5 \mathrm{~mL}$ THF rinse. The mixture was allowed to warm to room temperature and stirred for $c a$. 1 hour. The reaction was poured into a separatory funnel containing $250 \mathrm{~mL}$ saturated aqueous ammonium chloride and $250 \mathrm{~mL} \mathrm{Et}_{2} \mathrm{O}$ was added. The layers were separated and the aqueous layer was washed with $100 \mathrm{~mL} \mathrm{Et}_{2} \mathrm{O}$. The combined organics were washed with brine, dried with sodium sulfate, filtered and concentrated in-vacuo to afford a yellow oil. Purification by flash chromatography (25\% $\mathrm{Et}_{2} \mathrm{O}$ in hexanes) provided olefin SI-12 (2.46 g, $8.03 \mathrm{mmol}$, 99\% yield).

Note 1 - A heat gun was used on the low setting to dry the phosphonium salt, the salt was agitated during this process to prevent melting.

Note 2 - Brine + ice was used, temperature was monitored using a probe.

Physical state: colorless oil

TLC: $\mathrm{R}_{f}=0.3\left(25 \% \mathrm{Et}_{2} \mathrm{O}\right.$ in hexanes; visualization by $\left.\mathrm{UV}, \mathrm{KMnO}_{4}\right)$

$[\alpha]_{D}^{20.0}=-2.4\left(c=1.0, \mathrm{CHCl}_{3}\right)$

${ }^{1}$ H NMR (500 MHz, CDCl $): \delta 7.87(\mathrm{~d}, J=7.5 \mathrm{~Hz}, 2 \mathrm{H}), 7.63(\mathrm{t}, J=7.4 \mathrm{~Hz}, 1 \mathrm{H}), 7.54(\mathrm{t}, J=7.7$ $\mathrm{Hz}, 2 \mathrm{H}), 5.37(\mathrm{dt}, J=17.2,9.7 \mathrm{~Hz}, 1 \mathrm{H}), 4.76(\mathrm{~d}, J=17.2 \mathrm{~Hz}, 1 \mathrm{H}), 4.72(\mathrm{dd}, J=10.1,1.2 \mathrm{~Hz}, 1 \mathrm{H})$, $3.04-2.95(\mathrm{~m}, 2 \mathrm{H}), 1.85-1.78(\mathrm{~m}, 1 \mathrm{H}), 1.76-1.66(\mathrm{~m}, 2 \mathrm{H}), 1.62-1.54(\mathrm{~m}, 2 \mathrm{H}), 1.42-1.34$ $(\mathrm{m}, 1 \mathrm{H}), 1.21-1.12(\mathrm{~m}, 1 \mathrm{H}), 0.86-0.75(\mathrm{~m}, 2 \mathrm{H}), 0.82(\mathrm{~d}, J=6.5 \mathrm{~Hz}, 3 \mathrm{H}), 0.77(\mathrm{~d}, J=6.4 \mathrm{~Hz}$, $3 \mathrm{H}), 0.64(\mathrm{q}, J=12.0 \mathrm{~Hz}, 1 \mathrm{H})$.

${ }^{13}$ C NMR (125 MHz, $\left.\mathbf{C D C l}_{3}\right): \delta$ 142.2, 138.9, 133.6, 129.2, 128.3, 114.4, 51.7, 46.3, 45.6, 44.4, $42.4,33.9,31.8,22.4,22.3,19.9$.

HRMS (m/z): calculated for $\mathrm{C}_{18} \mathrm{H}_{27} \mathrm{O}_{2} \mathrm{~S}[\mathrm{M}+\mathrm{H}]^{+}:$307.1726, found 307.1733 . 

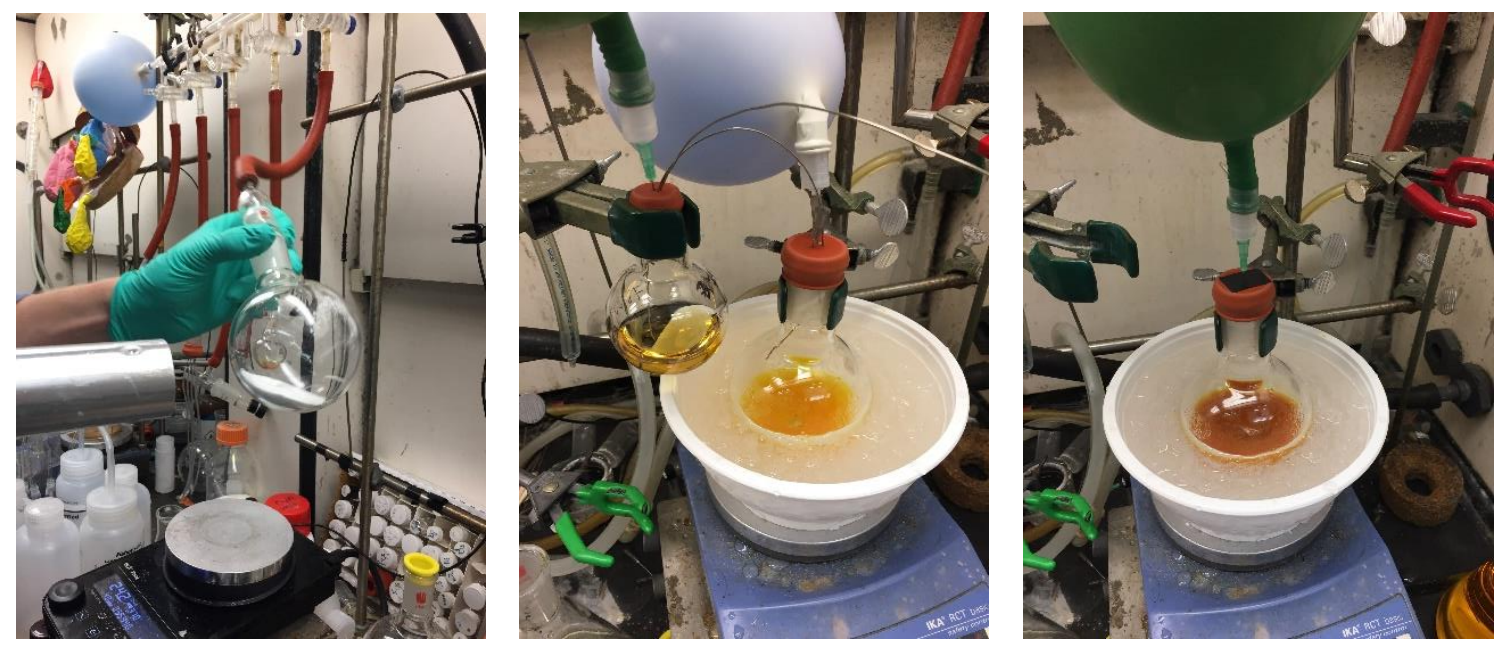

(left) drying methyltriphenylphosphonium bromide; (middle) cannula transfer of aldehyde to phosphonium ylide; (right) reaction mixture after aldehyde addition.

\section{Compound 15}

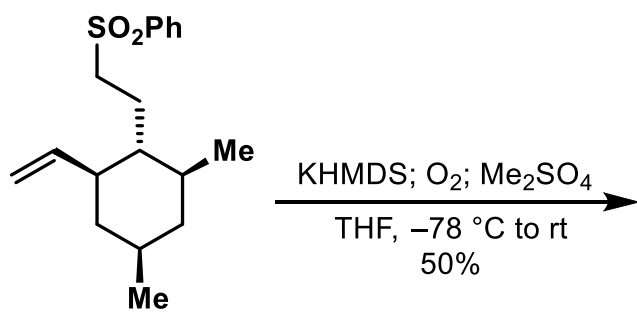

SI-12<smiles>C=CC1CC(C)CC(C)[C@H]1CC(=O)OC</smiles>

15

To a flame dried $100 \mathrm{~mL}$ round-bottomed flask equipped with large stir bar was added olefin SI12 (0.698 g, $2.28 \mathrm{mmol}, 1.00$ equiv.) and anhydrous THF (27 mL, $0.085 \mathrm{M})$. The mixture was cooled to $-78{ }^{\circ} \mathrm{C}$ and a $1 \mathrm{M}$ solution of KHMDS in THF (5.7 mL, $5.70 \mathrm{mmol}, 2.50$ equiv.) was added dropwise; the resulting bright yellow solution was allowed to stir 30 minutes at this temperature. The flask was then fitted with an 18-gauge vent needle attached to an oil bubbler then, an oxygen balloon bearing a long 18-gauge needle was submerged in the reaction mixture and vigorous stirring was applied (Note 1). Oxygen was bubbled through the reaction for 45 mins, until starting material was consumed by TLC (25\% $\mathrm{Et}_{2} \mathrm{O}$ in hexanes). Upon completion, the oxygen balloon and vent needle were removed and the reaction was warmed to room temperature. Dimethyl sulfate $(0.76 \mathrm{~mL}, 7.98 \mathrm{mmol}, 3.50$ equiv.) (Note 2) was added and the mixture was stirred vigorously for 1 hour (Note 3$)$. Morpholine $(0.6 \mathrm{~mL}, 6.84 \mathrm{mmol}, 3.00$ equiv.) was then added and stirring was continued for an additional $30 \mathrm{mins}$ to destroy residual dimethyl sulfate (Note 4). The reaction was quenched with saturated aqueous sodium sulfite ( $3 \mathrm{~mL})$, transferred to a separatory funnel and diluted with $\mathrm{Et}_{2} \mathrm{O}(150 \mathrm{~mL})$. The organic layer was washed with $1 \mathrm{M} \mathrm{HCl}$ $(100 \mathrm{~mL})$, half saturated aqueous sodium bisulfite $(100 \mathrm{~mL})$, water $(100 \mathrm{~mL})$ and brine $(100 \mathrm{~mL})$. The organic layer was dried with sodium sulfate, filtered and concentrated in-vacuo (Note 5) to 
afford an oily residue which was purified by flash chromatography (4\% $\mathrm{Et}_{2} \mathrm{O}$ in hexanes) to afford ester 15 (0.242 mg, $1.15 \mathrm{mmol}, 50 \%$ yield) (Note 6).

Note 1 - A high flow rate is required for efficient conversion. It was found that over time, the tip of the needle iced-over, reducing the flow rate; this could be remedied by pulling the needle into the upper headspace of the flask and allowing it to thaw before re-submerging. Pure oxygen and THF can form explosive peroxides, the reaction was conducted behind a blast as a precautionary measure.

Note 2 - CAUTION, dimethyl sulfate is highly toxic and should only be handled in a fume hood.

Note 3 - The reaction mixture becomes quite thick after addition of dimethyl sulfate, a large stir bar and vigorous stirring are required to obtain adequate mixing; the mixture thins out over the course of 1 hour.

Note 4 - No dimethyl sulfate was detected in the crude NMR.

Note 5 - Solvent was removed using a rotary evaporator in a fume hood.

Note 6 - An aldehyde by-product resulting from incomplete oxidation was observed in some instances following workup. This compound was co-polar with the desired product and difficult to separate chromatographically. A DMF-based extraction procedure was effective at removing this impurity without any product loss. ${ }^{12}$

Physical state: colorless oil

TLC: $\mathrm{R}_{f}=0.4\left(5 \% \mathrm{Et}_{2} \mathrm{O}\right.$ in hexanes $)$

$[\alpha]_{D}^{20.0}=+9.6\left(c=1.0, \mathrm{CHCl}_{3}\right)$

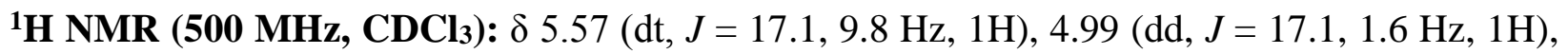
$4.94(\mathrm{dd}, J=10.2,2.0 \mathrm{~Hz}, 1 \mathrm{H}), 3.62(\mathrm{~s}, 3 \mathrm{H}), 2.41(\mathrm{dd}, J=16.1,4.4 \mathrm{~Hz}, 1 \mathrm{H}), 2.30$ (dd, $J=16.1$, $4.9 \mathrm{~Hz}, 1 \mathrm{H}), 1.95-1.89(\mathrm{~m}, 1 \mathrm{H}), 1.63(\mathrm{~d}, J=13.1 \mathrm{~Hz}, 2 \mathrm{H}), 1.53-1.41(\mathrm{~m}, 1 \mathrm{H}), 1.33-1.25(\mathrm{~m}$, $2 \mathrm{H}), 0.91-0.85(\mathrm{~m}, 1 \mathrm{H}), 0.90(\mathrm{~d}, J=6.1 \mathrm{~Hz}, 3 \mathrm{H}), 0.87(\mathrm{~d}, J=6.5 \mathrm{~Hz}, 3 \mathrm{H}), 0.74(\mathrm{q}, J=11.7 \mathrm{~Hz}$, $1 \mathrm{H})$.

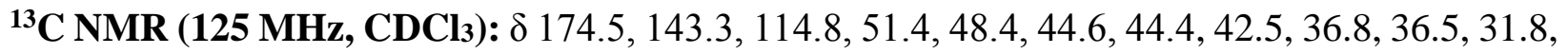
22.6, 20.5.

HRMS (m/z): calculated for $\mathrm{C}_{13} \mathrm{H}_{23} \mathrm{O}_{2}[\mathrm{M}+\mathrm{H}]^{+}:$211.1698, found 211.1694. 

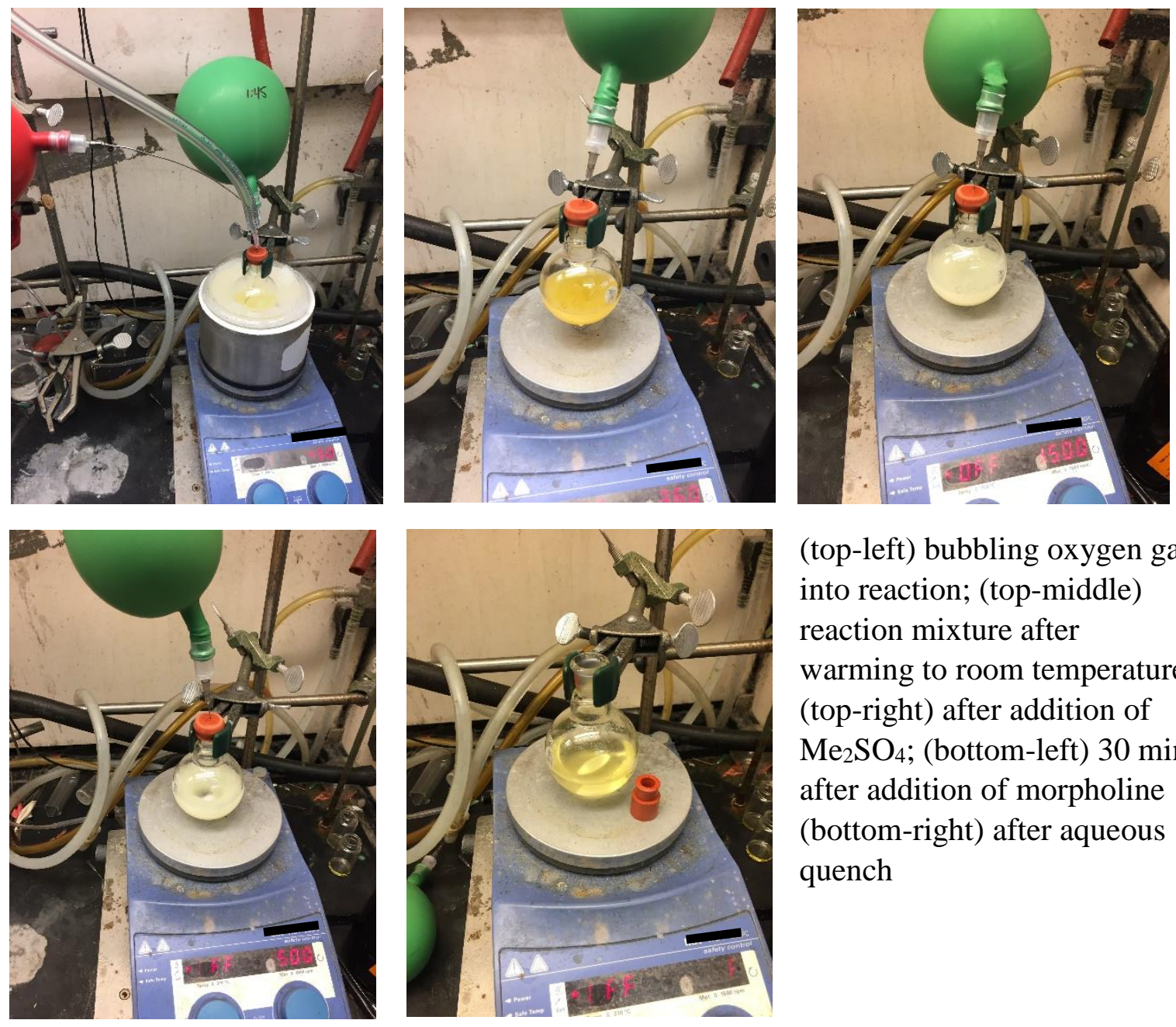

(top-left) bubbling oxygen gas into reaction; (top-middle) reaction mixture after warming to room temperature; (top-right) after addition of $\mathrm{Me}_{2} \mathrm{SO}_{4}$; (bottom-left) $30 \mathrm{mins}$ after addition of morpholine (bottom-right) after aqueous quench

\section{Compound 6}

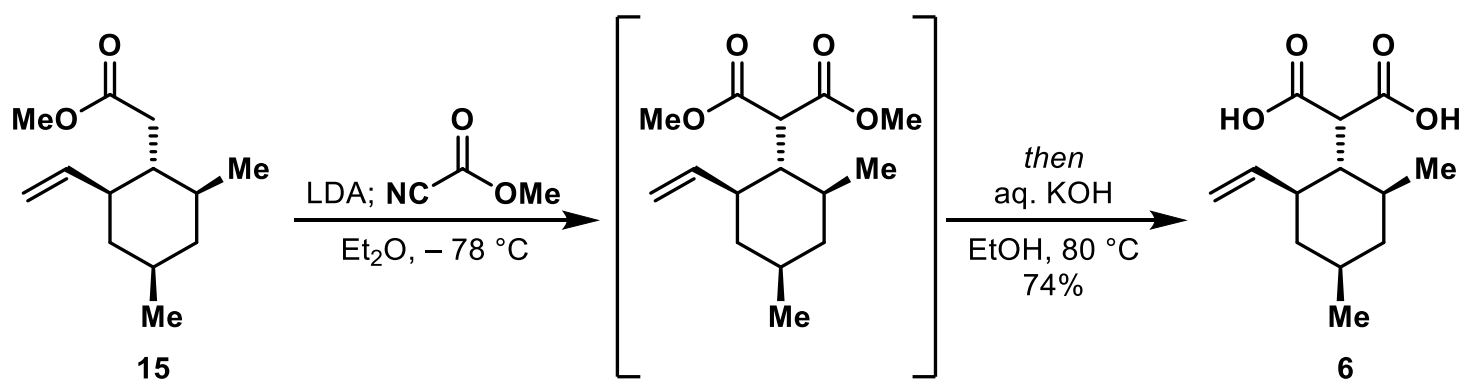

A flame dried $50 \mathrm{~mL}$ round-bottomed flask was charged with $\mathrm{Et}_{2} \mathrm{O}(6 \mathrm{~mL})$ and cooled to $-78{ }^{\circ} \mathrm{C}$ before $0.5 \mathrm{M}$ LDA in $\mathrm{Et}_{2} \mathrm{O}$ (9.5 mL, $4.77 \mathrm{mmol}, 3.00$ equiv.) was added (Note 1). Ester 15 (0.334 $\mathrm{g}, 1.59 \mathrm{mmol}, 1.00$ equiv.) dissolved in $\mathrm{Et}_{2} \mathrm{O}(6 \mathrm{~mL})$ was added dropwise to the LDA solution and the donor flask was rinsed with $\mathrm{Et}_{2} \mathrm{O}(4 \mathrm{~mL})$. The mixture was warmed to $0{ }^{\circ} \mathrm{C}$ and stirred for 1 hour before cooling back to $-78^{\circ} \mathrm{C}$. Methyl cyanoformate $(0.5 \mathrm{~mL}, 6.36 \mathrm{mmol}, 4.00$ equiv.) (Note 2) was added dropwise and the heterogeneous yellow solution was allowed to stir for 1 hour. The 
reaction was quenched at $-78{ }^{\circ} \mathrm{C}$ by the addition of methanol $(0.5 \mathrm{~mL})$ (Note 3$)$ and allowed to warm to room temperature. The orange solution was concentrated under reduced pressure (Note 4) to afford a foamy orange solid which was re-suspended in ethanol (17 mL). Aqueous $\mathrm{KOH}(3$ $\mathrm{M}, 4.2 \mathrm{~mL}$ ) was added and the flask was fitted with a reflux condenser before being transferred to an oil bath pre-heated to $80{ }^{\circ} \mathrm{C}$. The mixture was stirred vigorously (1100 rpm) for $12 \mathrm{hrs}$ then cooled to room temperature and concentrated to remove most of the ethanol (Note 5). The aqueous residue was treated with $5 \mathrm{~mL} 3 \mathrm{~N} \mathrm{NaOH}$ and diluted with $50 \mathrm{~mL} \mathrm{H}_{2} \mathrm{O}$ before being transferred to a separatory funnel. The aqueous layer was extracted with $\mathrm{Et}_{2} \mathrm{O}(2 \mathrm{X} 25 \mathrm{~mL})$, then acidified using $2 \mathrm{~N} \mathrm{HCl}$ to $\sim \mathrm{pH} 2$ (Note 6). The aqueous layer was extracted with ethyl acetate $(4 \mathrm{X} 50 \mathrm{~mL}$ ) and the combined organics were dried with sodium sulfate, filtered and concentrated in-vacuo (Note 7). The resulting pale yellow solid was subjected to trituration ( $30 \%$ hexanes in $\mathrm{CH}_{2} \mathrm{Cl}_{2}$ ) to afford a white crystalline solid corresponding to pure 6 ( $0.284 \mathrm{~g}, 1.18 \mathrm{mmol}, 74 \%$ yield).

Note 1 - LDA was freshly prepared from diisopropylamine and $n$-butyl lithium.

Note 2 - CAUTION Methyl cyanoformate (Mander's reagent) is highly toxic and should only be handled in a fume hood.

Note 3 - Squirt bottle methanol was used.

Note 4 - Solvent was removed using a rotary evaporator in a fume hood.

Note 5 - Solvent was removed using a rotary evaporator in a fume hood.

Note 6 - CAUTION acidification of the aqueous layer produces hydrogen cyanide, this operation was performed in a well-functioning fume hood. All workup solvents should be quenched with bleach and disposed of appropriately.

Note 7 - Solvent was removed using a rotary evaporator in a fume hood.

Physical state: white crystaline solid.

TLC: $\mathrm{R}_{f}=0.05\left(10 \% \mathrm{MeOH}\right.$ in $\mathrm{CH}_{2} \mathrm{Cl}_{2}$; visualization by $\left.\mathrm{KMnO}_{4}\right)$

m.p.: $152-153{ }^{\circ} \mathrm{C}$

$[\alpha]_{D}^{20.0}=-11.5(c=1.0, \mathrm{MeOH})$

${ }^{1}$ H NMR (600 MHz, Methanol-d4): $\delta 5.61(\mathrm{dt}, J=17.2,9.8 \mathrm{~Hz}, 1 \mathrm{H}), 5.08(\mathrm{dd}, J=17.2,1.8 \mathrm{~Hz}$, $1 \mathrm{H}), 5.00(\mathrm{dd}, J=10.2,2.0 \mathrm{~Hz}, 1 \mathrm{H}), 3.72(\mathrm{~d}, J=1.9 \mathrm{~Hz}, 1 \mathrm{H}), 2.32-2.23(\mathrm{~m}, 1 \mathrm{H}), 1.79-1.68(\mathrm{~m}$, 2H), $1.65(\mathrm{~d}, J=13.0 \mathrm{~Hz}, 2 \mathrm{H}), 1.53-1.42(\mathrm{~m}, 1 \mathrm{H}), 0.98(\mathrm{~d}, J=6.2 \mathrm{~Hz}, 3 \mathrm{H}), 0.95-0.86(\mathrm{~m}, 4 \mathrm{H})$, $0.77(\mathrm{q}, J=11.8 \mathrm{~Hz}, 1 \mathrm{H})$.

${ }^{13}$ C NMR (150 MHz, Methanol-d4): $\delta$ 174.3, 173.1, 143.8, 116.5, 52.6, 49.3, 47.5, 46.2, 44.0, $35.4,32.8,22.8,21.3$.

HRMS (m/z): calculated for $\mathrm{C}_{13} \mathrm{H}_{21} \mathrm{O}_{4}[\mathrm{M}+\mathrm{H}]^{+}:$241.1440, found 241.1438. 


\section{X-ray Structure 6:}

\section{Experimental Summary}

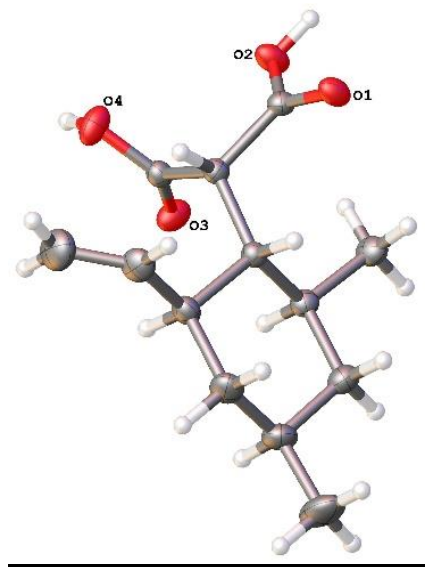

The single crystal X-ray diffraction studies were carried out on a Bruker SMART APEX II CCD diffractometer equipped with $\mathrm{Cu} \mathrm{K}$ radiation $(\lambda=1.54178)$. Crystals of the subject compound were used as received (grown by vapor diffusion of hexane into ethyl acetate). A 0.140 x 0.140 x $0.140 \mathrm{~mm}$ translucent, colorless block crystal was mounted on a Cryoloop with Paratone-N oil.

Data were collected in a nitrogen gas stream at 100(2) K using $\phi$ and $\varpi$ scans. Crystal-to-detector distance was $40 \mathrm{~mm}$ using exposure times of $0.5,1$ and 3 seconds (depending on the $2 \theta$ position) with a scan width of $1.50^{\circ}$. Data collection was $99.9 \%$ complete to $67.679^{\circ}$ in $\theta$. A total of 12788 reflections were collected. 2604 reflections were found to be symmetry independent, with an $\mathrm{R}_{\text {int }}$ of 0.0297 . Indexing and unit cell refinement indicated a Primitive Trigonal lattice. The space group was found to be $\boldsymbol{P 3 _ { 1 }}$. The data were integrated using the Bruker SAINT Software program and scaled using the SADABS software program. Solution by direct methods (SHELXT) produced a complete phasing model consistent with the proposed structure.

All non-hydrogen atoms were refined anisotropically by full-matrix least-squares (SHELXL-2014). All carbon bonded hydrogen atoms were placed using a riding model. Their positions were constrained relative to their parent atom using the appropriate HFIX command in SHELXL-2014.

Notes: Excellent data and refinement. Absolute stereochemistry established (Flack parameter, 0.04(5)).

Table 6. Crystal data and structure refinement for Baran744.

Report date

Identification code

Empirical formula

Formula weight

Temperature

Wavelength

Crystal system

Space group

Unit cell dimensions

Volume

$\mathrm{Z}$

Density (calculated)

Absorption coefficient

$\mathrm{F}(000)$

Crystal size
2019-10-15

Baran744

C13 $\mathrm{H} 20 \mathrm{O} 4$

240.29

$100.0 \mathrm{~K}$

$1.54178 \AA$

Trigonal

P31

$\mathrm{a}=9.34920(10) \AA \quad \alpha=90^{\circ}$.

$\mathrm{b}=9.34920(10) \AA \quad \beta=90^{\circ}$.

$\mathrm{c}=13.5555(2) \AA \quad \gamma=120^{\circ}$.

1026.11(3) $\AA^{3}$

3

$1.167 \mathrm{Mg} / \mathrm{m}^{3}$

$0.701 \mathrm{~mm}^{-1}$

390

$0.14 \times 0.14 \times 0.14 \mathrm{~mm}^{3}$ 
Theta range for data collection

Index ranges

Reflections collected

Independent reflections

Completeness to theta $=67.679^{\circ}$

Absorption correction

Max. and min. transmission

Refinement method

Data / restraints / parameters

Goodness-of-fit on $\mathrm{F}^{2}$

Final R indices [I $>2 \operatorname{sigma}(\mathrm{I})]$

$\mathrm{R}$ indices (all data)

Absolute structure parameter

Extinction coefficient

Largest diff. peak and hole
5.463 to $69.958^{\circ}$.

$-11<=\mathrm{h}<=11,-11<=\mathrm{k}<=11,-16<=\mathrm{l}<=16$

12778

2604 [R(int $)=0.0297]$

$99.9 \%$

Semi-empirical from equivalents

0.7533 and 0.6731

Full-matrix least-squares on $\mathrm{F}^{2}$

2604 / 1 / 161

1.065

$\mathrm{R} 1=0.0253, \mathrm{wR} 2=0.0665$

$\mathrm{R} 1=0.0256, \mathrm{wR} 2=0.0670$

$0.04(5)$

$0.0024(8)$

0.216 and -0.120 e. $\AA^{-3}$

Table 7. Atomic coordinates ( $\mathrm{x} 10^{4}$ ) and equivalent isotropic displacement parameters $\left(\AA^{2} \times 10^{3}\right)$ for Baran744. $\mathrm{U}(\mathrm{eq})$ is defined as one third of the trace of the orthogonalized $\mathrm{U}^{\mathrm{ij}}$ tensor.

\begin{tabular}{|c|c|c|c|c|}
\hline & $\mathrm{x}$ & $\mathrm{y}$ & $\mathrm{z}$ & $\mathrm{U}(\mathrm{eq})$ \\
\hline $\mathrm{O}(1)$ & $5308(2)$ & $5253(1)$ & $6506(1)$ & $26(1)$ \\
\hline $\mathrm{O}(2)$ & $6028(2)$ & $3785(2)$ & $5534(1)$ & $28(1)$ \\
\hline $\mathrm{O}(3)$ & $7050(2)$ & $5597(2)$ & $3495(1)$ & $29(1)$ \\
\hline $\mathrm{O}(4)$ & $9213(2)$ & $6291(2)$ & $4485(1)$ & $32(1)$ \\
\hline $\mathrm{C}(1)$ & $6177(2)$ & $7594(2)$ & $4876(1)$ & $19(1)$ \\
\hline $\mathrm{C}(2)$ & $4736(2)$ & $6773(2)$ & $4138(1)$ & $22(1)$ \\
\hline $\mathrm{C}(3)$ & $3920(2)$ & $7841(2)$ & $4049(1)$ & $28(1)$ \\
\hline $\mathrm{C}(4)$ & $5120(2)$ & $9600(2)$ & $3716(2)$ & $34(1)$ \\
\hline $\mathrm{C}(5)$ & $6595(2)$ & $10395(2)$ & $4415(2)$ & $32(1)$ \\
\hline $\mathrm{C}(6)$ & $7456(2)$ & $9367(2)$ & $4539(1)$ & $25(1)$ \\
\hline $\mathrm{C}(7)$ & $3440(2)$ & $5002(2)$ & $4417(1)$ & $28(1)$ \\
\hline $\mathrm{C}(8)$ & $4247(3)$ & $10613(3)$ & $3658(2)$ & $54(1)$ \\
\hline $\mathrm{C}(9)$ & $8850(2)$ & $10213(2)$ & $5263(2)$ & $32(1)$ \\
\hline$C(10)$ & $10418(3)$ & $10747(3)$ & $5079(2)$ & $47(1)$ \\
\hline $\mathrm{C}(11)$ & $7063(2)$ & $6617(2)$ & $5134(1)$ & $20(1)$ \\
\hline$C(12)$ & $6021(2)$ & $5134(2)$ & $5787(1)$ & $20(1)$ \\
\hline$C(13)$ & $7765(2)$ & $6122(2)$ & $4278(1)$ & 21(1) \\
\hline
\end{tabular}

Table 8. Bond lengths $[\AA]$ and angles $\left[{ }^{\circ}\right]$ for Baran744.

\begin{tabular}{llll}
\hline $\mathrm{O}(1)-\mathrm{C}(12)$ & $1.217(2)$ & $\mathrm{C}(3)-\mathrm{H}(3 \mathrm{~A})$ & 0.9900 \\
$\mathrm{O}(2)-\mathrm{H}(2)$ & $0.88(3)$ & $\mathrm{C}(3)-\mathrm{H}(3 \mathrm{~B})$ & 0.9900 \\
$\mathrm{O}(2)-\mathrm{C}(12)$ & $1.3102(19)$ & $\mathrm{C}(3)-\mathrm{C}(4)$ & $1.524(2)$ \\
$\mathrm{O}(3)-\mathrm{C}(13)$ & $1.218(2)$ & $\mathrm{C}(4)-\mathrm{H}(4 \mathrm{~A})$ & 1.0000 \\
$\mathrm{O}(4)-\mathrm{H}(4)$ & $0.91(3)$ & $\mathrm{C}(4)-\mathrm{C}(5)$ & $1.525(3)$ \\
$\mathrm{O}(4)-\mathrm{C}(13)$ & $1.312(2)$ & $\mathrm{C}(4)-\mathrm{C}(8)$ & $1.531(3)$ \\
$\mathrm{C}(1)-\mathrm{H}(1)$ & 1.0000 & $\mathrm{C}(5)-\mathrm{H}(5 \mathrm{~A})$ & 0.9900 \\
$\mathrm{C}(1)-\mathrm{C}(2)$ & $1.540(2)$ & $\mathrm{C}(5)-\mathrm{H}(5 \mathrm{~B})$ & 0.9900 \\
$\mathrm{C}(1)-\mathrm{C}(6)$ & $1.550(2)$ & $\mathrm{C}(5)-\mathrm{C}(6)$ & $1.541(2)$ \\
$\mathrm{C}(1)-\mathrm{C}(11)$ & $1.549(2)$ & $\mathrm{C}(6)-\mathrm{H}(6)$ & 1.0000 \\
$\mathrm{C}(2)-\mathrm{H}(2 \mathrm{~A})$ & 1.0000 & $\mathrm{C}(6)-\mathrm{C}(9)$ & $1.502(2)$ \\
$\mathrm{C}(2)-\mathrm{C}(3)$ & $1.534(2)$ & $\mathrm{C}(7)-\mathrm{H}(7 \mathrm{~A})$ & 0.9800 \\
$\mathrm{C}(2)-\mathrm{C}(7)$ & $1.532(2)$ & $\mathrm{C}(7)-\mathrm{H}(7 \mathrm{~B})$ & 0.9800
\end{tabular}




\begin{tabular}{|c|c|c|c|}
\hline $\mathrm{C}(7)-\mathrm{H}(7 \mathrm{C})$ & 0.9800 & $\mathrm{H}(5 \mathrm{~A})-\mathrm{C}(5)-\mathrm{H}(5 \mathrm{~B})$ & 107.8 \\
\hline $\mathrm{C}(8)-\mathrm{H}(8 \mathrm{~A})$ & 0.9800 & $\mathrm{C}(6)-\mathrm{C}(5)-\mathrm{H}(5 \mathrm{~A})$ & 109.0 \\
\hline $\mathrm{C}(8)-\mathrm{H}(8 \mathrm{~B})$ & 0.9800 & $\mathrm{C}(6)-\mathrm{C}(5)-\mathrm{H}(5 \mathrm{~B})$ & 109.0 \\
\hline $\mathrm{C}(8)-\mathrm{H}(8 \mathrm{C})$ & 0.9800 & $\mathrm{C}(1)-\mathrm{C}(6)-\mathrm{H}(6)$ & 108.7 \\
\hline $\mathrm{C}(9)-\mathrm{H}(9)$ & 0.9500 & $C(5)-C(6)-C(1)$ & $109.57(13)$ \\
\hline $\mathrm{C}(9)-\mathrm{C}(10)$ & $1.315(3)$ & $\mathrm{C}(5)-\mathrm{C}(6)-\mathrm{H}(6)$ & 108.7 \\
\hline $\mathrm{C}(10)-\mathrm{H}(10 \mathrm{~A})$ & 0.9500 & $C(9)-C(6)-C(1)$ & $111.89(14)$ \\
\hline $\mathrm{C}(10)-\mathrm{H}(10 \mathrm{~B})$ & 0.9500 & $\mathrm{C}(9)-\mathrm{C}(6)-\mathrm{C}(5)$ & $109.23(14)$ \\
\hline $\mathrm{C}(11)-\mathrm{H}(11)$ & 1.0000 & $\mathrm{C}(9)-\mathrm{C}(6)-\mathrm{H}(6)$ & 108.7 \\
\hline $\mathrm{C}(11)-\mathrm{C}(12)$ & $1.518(2)$ & $\mathrm{C}(2)-\mathrm{C}(7)-\mathrm{H}(7 \mathrm{~A})$ & 109.5 \\
\hline $\mathrm{C}(11)-\mathrm{C}(13)$ & $1.516(2)$ & $\mathrm{C}(2)-\mathrm{C}(7)-\mathrm{H}(7 \mathrm{~B})$ & 109.5 \\
\hline $\mathrm{C}(12)-\mathrm{O}(2)-\mathrm{H}(2)$ & 109.5 & $\mathrm{C}(2)-\mathrm{C}(7)-\mathrm{H}(7 \mathrm{C})$ & 109.5 \\
\hline $\mathrm{C}(13)-\mathrm{O}(4)-\mathrm{H}(4)$ & 109.5 & $\mathrm{H}(7 \mathrm{~A})-\mathrm{C}(7)-\mathrm{H}(7 \mathrm{~B})$ & 109.5 \\
\hline $\mathrm{C}(2)-\mathrm{C}(1)-\mathrm{H}(1)$ & 106.3 & $\mathrm{H}(7 \mathrm{~A})-\mathrm{C}(7)-\mathrm{H}(7 \mathrm{C})$ & 109.5 \\
\hline $\mathrm{C}(2)-\mathrm{C}(1)-\mathrm{C}(6)$ & $110.88(12)$ & $\mathrm{H}(7 \mathrm{~B})-\mathrm{C}(7)-\mathrm{H}(7 \mathrm{C})$ & 109.5 \\
\hline $\mathrm{C}(2)-\mathrm{C}(1)-\mathrm{C}(11)$ & $116.42(12)$ & $\mathrm{C}(4)-\mathrm{C}(8)-\mathrm{H}(8 \mathrm{~A})$ & 109.5 \\
\hline $\mathrm{C}(6)-\mathrm{C}(1)-\mathrm{H}(1)$ & 106.3 & $\mathrm{C}(4)-\mathrm{C}(8)-\mathrm{H}(8 \mathrm{~B})$ & 109.5 \\
\hline $\mathrm{C}(11)-\mathrm{C}(1)-\mathrm{H}(1)$ & 106.3 & $\mathrm{C}(4)-\mathrm{C}(8)-\mathrm{H}(8 \mathrm{C})$ & 109.5 \\
\hline C(11)-C(1)-C(6) & $110.05(12)$ & $\mathrm{H}(8 \mathrm{~A})-\mathrm{C}(8)-\mathrm{H}(8 \mathrm{~B})$ & 109.5 \\
\hline $\mathrm{C}(1)-\mathrm{C}(2)-\mathrm{H}(2 \mathrm{~A})$ & 108.1 & $\mathrm{H}(8 \mathrm{~A})-\mathrm{C}(8)-\mathrm{H}(8 \mathrm{C})$ & 109.5 \\
\hline $\mathrm{C}(3)-\mathrm{C}(2)-\mathrm{C}(1)$ & $108.80(13)$ & $\mathrm{H}(8 \mathrm{~B})-\mathrm{C}(8)-\mathrm{H}(8 \mathrm{C})$ & 109.5 \\
\hline $\mathrm{C}(3)-\mathrm{C}(2)-\mathrm{H}(2 \mathrm{~A})$ & 108.1 & $\mathrm{C}(6)-\mathrm{C}(9)-\mathrm{H}(9)$ & 117.1 \\
\hline $\mathrm{C}(7)-\mathrm{C}(2)-\mathrm{C}(1)$ & $113.61(13)$ & $\mathrm{C}(10)-\mathrm{C}(9)-\mathrm{C}(6)$ & $125.8(2)$ \\
\hline $\mathrm{C}(7)-\mathrm{C}(2)-\mathrm{H}(2 \mathrm{~A})$ & 108.1 & $\mathrm{C}(10)-\mathrm{C}(9)-\mathrm{H}(9)$ & 117.1 \\
\hline $\mathrm{C}(7)-\mathrm{C}(2)-\mathrm{C}(3)$ & $109.92(13)$ & $\mathrm{C}(9)-\mathrm{C}(10)-\mathrm{H}(10 \mathrm{~A})$ & 120.0 \\
\hline $\mathrm{C}(2)-\mathrm{C}(3)-\mathrm{H}(3 \mathrm{~A})$ & 109.0 & $\mathrm{C}(9)-\mathrm{C}(10)-\mathrm{H}(10 \mathrm{~B})$ & 120.0 \\
\hline $\mathrm{C}(2)-\mathrm{C}(3)-\mathrm{H}(3 \mathrm{~B})$ & 109.0 & $\mathrm{H}(10 \mathrm{~A})-\mathrm{C}(10)-\mathrm{H}(10 \mathrm{~B})$ & 120.0 \\
\hline $\mathrm{H}(3 \mathrm{~A})-\mathrm{C}(3)-\mathrm{H}(3 \mathrm{~B})$ & 107.8 & $\mathrm{C}(1)-\mathrm{C}(11)-\mathrm{H}(11)$ & 105.5 \\
\hline $\mathrm{C}(4)-\mathrm{C}(3)-\mathrm{C}(2)$ & $112.82(14)$ & $\mathrm{C}(12)-\mathrm{C}(11)-\mathrm{C}(1)$ & $112.48(12)$ \\
\hline $\mathrm{C}(4)-\mathrm{C}(3)-\mathrm{H}(3 \mathrm{~A})$ & 109.0 & $\mathrm{C}(12)-\mathrm{C}(11)-\mathrm{H}(11)$ & 105.5 \\
\hline $\mathrm{C}(4)-\mathrm{C}(3)-\mathrm{H}(3 \mathrm{~B})$ & 109.0 & $\mathrm{C}(13)-\mathrm{C}(11)-\mathrm{C}(1)$ & $116.66(13)$ \\
\hline $\mathrm{C}(3)-\mathrm{C}(4)-\mathrm{H}(4 \mathrm{~A})$ & 108.4 & $\mathrm{C}(13)-\mathrm{C}(11)-\mathrm{H}(11)$ & 105.5 \\
\hline C(3)-C(4)-C(5) & 109.90(14) & $\mathrm{C}(13)-\mathrm{C}(11)-\mathrm{C}(12)$ & $110.23(13)$ \\
\hline C(3)-C(4)-C(8) & $110.49(16)$ & $\mathrm{O}(1)-\mathrm{C}(12)-\mathrm{O}(2)$ & $124.41(15)$ \\
\hline $\mathrm{C}(5)-\mathrm{C}(4)-\mathrm{H}(4 \mathrm{~A})$ & 108.4 & $\mathrm{O}(1)-\mathrm{C}(12)-\mathrm{C}(11)$ & $120.94(13)$ \\
\hline $\mathrm{C}(5)-\mathrm{C}(4)-\mathrm{C}(8)$ & $111.21(18)$ & $\mathrm{O}(2)-\mathrm{C}(12)-\mathrm{C}(11)$ & $114.59(13)$ \\
\hline $\mathrm{C}(8)-\mathrm{C}(4)-\mathrm{H}(4 \mathrm{~A})$ & 108.4 & $\mathrm{O}(3)-\mathrm{C}(13)-\mathrm{O}(4)$ & $124.10(15)$ \\
\hline $\mathrm{C}(4)-\mathrm{C}(5)-\mathrm{H}(5 \mathrm{~A})$ & 109.0 & $\mathrm{O}(3)-\mathrm{C}(13)-\mathrm{C}(11)$ & $123.72(14)$ \\
\hline $\mathrm{C}(4)-\mathrm{C}(5)-\mathrm{H}(5 \mathrm{~B})$ & 109.0 & $\mathrm{O}(4)-\mathrm{C}(13)-\mathrm{C}(11)$ & $112.17(13)$ \\
\hline $\mathrm{C}(4)-\mathrm{C}(5)-\mathrm{C}(6)$ & $113.00(15)$ & & \\
\hline
\end{tabular}

Symmetry transformations used to generate equivalent atoms:

Table 9. Anisotropic displacement parameters $\left(\AA^{2} \times 10^{3}\right)$ for Baran744. The anisotropic displacement factor exponent takes the form: $-2 \pi^{2}\left[h^{2} a^{* 2} U^{11}+\ldots+2 h k a^{*} b^{*} U^{12}\right]$

\begin{tabular}{lcccccc}
\hline & $\mathrm{U}^{11}$ & $\mathrm{U}^{22}$ & $\mathrm{U}^{33}$ & $\mathrm{U}^{23}$ & $\mathrm{U}^{13}$ & $\mathrm{U}^{12}$ \\
\hline $\mathrm{O}(1)$ & $35(1)$ & $25(1)$ & $23(1)$ & $4(1)$ & $8(1)$ & $18(1)$ \\
$\mathrm{O}(2)$ & $40(1)$ & $22(1)$ & $26(1)$ & $4(1)$ & $9(1)$ & $19(1)$ \\
$\mathrm{O}(3)$ & $33(1)$ & $42(1)$ & $22(1)$ & $-5(1)$ & $-2(1)$ & $26(1)$ \\
$\mathrm{O}(4)$ & $25(1)$ & $43(1)$ & $32(1)$ & $-12(1)$ & $-3(1)$ & $21(1)$ \\
$\mathrm{C}(1)$ & $20(1)$ & $18(1)$ & $19(1)$ & $0(1)$ & $1(1)$ & $9(1)$ \\
$\mathrm{C}(2)$ & $23(1)$ & $23(1)$ & $21(1)$ & $-1(1)$ & $-1(1)$ & $12(1)$ \\
$\mathrm{C}(3)$ & $27(1)$ & $31(1)$ & $29(1)$ & $0(1)$ & $-3(1)$ & $17(1)$ \\
$\mathrm{C}(4)$ & $37(1)$ & $32(1)$ & $39(1)$ & $10(1)$ & $0(1)$ & $21(1)$ \\
& & & & SI-68 & &
\end{tabular}




\begin{tabular}{lllllll}
$\mathrm{C}(5)$ & $33(1)$ & $21(1)$ & $43(1)$ & $5(1)$ & $2(1)$ & $14(1)$ \\
$\mathrm{C}(6)$ & $25(1)$ & $20(1)$ & $28(1)$ & $3(1)$ & $4(1)$ & $10(1)$ \\
$\mathrm{C}(7)$ & $24(1)$ & $24(1)$ & $31(1)$ & $-1(1)$ & $-7(1)$ & $10(1)$ \\
$\mathrm{C}(8)$ & $50(1)$ & $38(1)$ & $82(2)$ & $10(1)$ & $-12(1)$ & $29(1)$ \\
$\mathrm{C}(9)$ & $30(1)$ & $19(1)$ & $40(1)$ & $-2(1)$ & $-3(1)$ & $8(1)$ \\
$\mathrm{C}(10)$ & $29(1)$ & $36(1)$ & $63(2)$ & $2(1)$ & $-5(1)$ & $7(1)$ \\
$\mathrm{C}(11)$ & $20(1)$ & $20(1)$ & $19(1)$ & $0(1)$ & $0(1)$ & $10(1)$ \\
$\mathrm{C}(12)$ & $22(1)$ & $21(1)$ & $19(1)$ & $0(1)$ & $-2(1)$ & $12(1)$ \\
$\mathrm{C}(13)$ & $22(1)$ & $19(1)$ & $22(1)$ & $2(1)$ & $3(1)$ & $10(1)$ \\
\hline
\end{tabular}

Table 10. Hydrogen coordinates ( $\left.\times 10^{4}\right)$ and isotropic displacement parameters $\left(\AA^{2} \times 10^{3}\right)$ for Baran744.

\begin{tabular}{lcccc}
\hline & $\mathrm{x}$ & $\mathrm{y}$ & $\mathrm{z}$ & $\mathrm{U}(\mathrm{eq})$ \\
\hline & & & & \\
$\mathrm{H}(2)$ & $5450(30)$ & $3000(30)$ & $5964(17)$ & 41 \\
$\mathrm{H}(4)$ & $9500(17)$ & $5800(30)$ & $4005(18)$ & 48 \\
$\mathrm{H}(1)$ & 5694 & 7717 & 5507 & 23 \\
$\mathrm{H}(2 \mathrm{~A})$ & 5200 & 6745 & 3478 & 27 \\
$\mathrm{H}(3 \mathrm{~A})$ & 2998 & 7321 & 3571 & 33 \\
$\mathrm{H}(3 \mathrm{~B})$ & 3450 & 7877 & 4697 & 33 \\
$\mathrm{H}(4 \mathrm{~A})$ & 5531 & 9554 & 3042 & 41 \\
$\mathrm{H}(5 \mathrm{~A})$ & 6216 & 10546 & 5068 & 39 \\
$\mathrm{H}(5 \mathrm{~B})$ & 7405 & 11502 & 4159 & 39 \\
$\mathrm{H}(6)$ & 7920 & 9300 & 3888 & 30 \\
$\mathrm{H}(7 \mathrm{~A})$ & 3053 & 4985 & 5091 & 41 \\
$\mathrm{H}(7 \mathrm{~B})$ & 3933 & 4292 & 4379 & 41 \\
$\mathrm{H}(7 \mathrm{C})$ & 2505 & 4594 & 3959 & 41 \\
$\mathrm{H}(8 \mathrm{~A})$ & 3360 & 10123 & 3167 & 80 \\
$\mathrm{H}(8 \mathrm{~B})$ & 5043 & 11750 & 3467 & 80 \\
$\mathrm{H}(8 \mathrm{C})$ & 3777 & 10615 & 4304 & 80 \\
$\mathrm{H}(9)$ & 8574 & 10376 & 5912 & 38 \\
$\mathrm{H}(10 \mathrm{~A})$ & 10751 & 10610 & 4440 & 56 \\
$\mathrm{H}(10 \mathrm{~B})$ & 11219 & 11271 & 5584 & 56 \\
$\mathrm{H}(11)$ & 8036 & 7382 & 5545 & 24 \\
\hline
\end{tabular}

Table 11. Hydrogen bonds for Baran744 [ $\left[\AA\right.$ and $\left.{ }^{\circ}\right]$.

\begin{tabular}{lcccc}
\hline $\mathrm{D}-\mathrm{H} \ldots \mathrm{A}$ & $\mathrm{d}(\mathrm{D}-\mathrm{H})$ & $\mathrm{d}(\mathrm{H} \ldots \mathrm{A})$ & $\mathrm{d}(\mathrm{D} \ldots \mathrm{A})$ & $<(\mathrm{DHA})$ \\
\hline $\mathrm{O}(2)-\mathrm{H}(2) \ldots \mathrm{O}(3) \# 1$ & 0.88 & 1.73 & $2.6136(17)$ & 177.1 \\
$\mathrm{O}(4)-\mathrm{H}(4) \ldots \mathrm{O}(1) \# 2$ & 0.91 & 1.71 & $2.6246(16)$ & 174.3 \\
\hline
\end{tabular}

Symmetry transformations used to generate equivalent atoms:

$\# 1-y+1, x-y, z+1 / 3 \quad \# 2-x+y+1,-x+1, z-1 / 3$ 


\section{Compound 17}

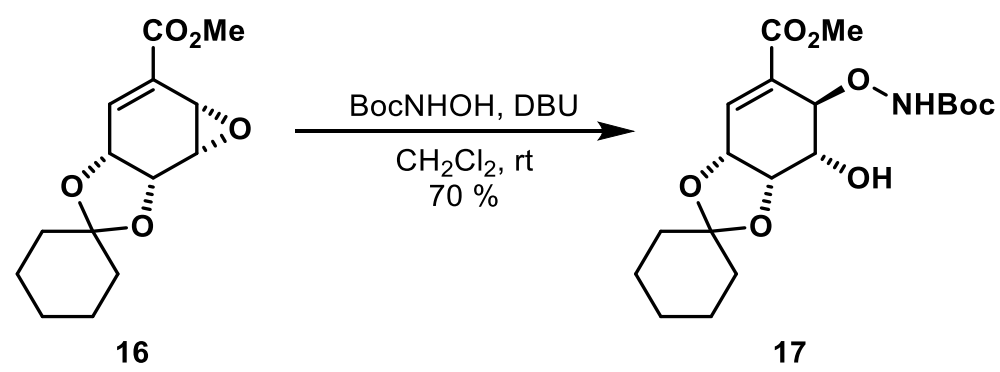

A flame dried $100 \mathrm{~mL}$ round-bottomed flask was charged with epoxide $\mathbf{1 6}^{13}(1.13 \mathrm{~g}, 4.25 \mathrm{mmol}$, 1.00 equiv.) and $\mathrm{CH}_{2} \mathrm{Cl}_{2}(21 \mathrm{~mL}, 0.2 \mathrm{M})$. A separate flame dried $50 \mathrm{~mL}$ round-bottomed flask containing $\mathrm{N}$-Boc-hydroxylamine $\left(0.678 \mathrm{~g}, 5.10 \mathrm{mmol}, 1.20\right.$ equiv.) was dissolved in $\mathrm{CH}_{2} \mathrm{Cl}_{2}$ (21 $\mathrm{mL}, 0.2 \mathrm{M})$ then treated with DBU $(0.63 \mathrm{~mL}, 4.25 \mathrm{mmol}, 1.00$ equiv. $)$. The $N$-Boc-hydroxylamine solution was added to the epoxide solution via rapid syringe transfer and the mixture was allowed to stir 5 mins (Note 1). The reaction mixture was poured into a separatory funnel containing 200 $\mathrm{mL}$ half saturated aqueous ammonium chloride and was diluted with $\mathrm{Et}_{2} \mathrm{O}(100 \mathrm{~mL})$. The layers were separated and the aqueous layer was extracted with an additional $100 \mathrm{~mL} \mathrm{Et}_{2} \mathrm{O}$. The combined organics were washed with brine $(150 \mathrm{~mL})$, dried with sodium sulfate and concentrated under reduced pressure. The oily residue was purified by flash chromatography (50\% $\mathrm{Et}_{2} \mathrm{O}$ in hexanes) to afford the title compound 17 (1.19 g, $2.98 \mathrm{mmol}, 70 \%$ yield $)$.

Note 1 - The reaction is complete nearly instantaneously, it can be monitored using $10 \%$ acetone in toluene. The major by-product is the diphenol, this impurity increases at the expense of product if the reaction is allowed to stir for an extended period.

Physical state: white crystalline solid

TLC: $\mathrm{R}_{f}=0.45\left(10 \%\right.$ acetone in toluene; visualization $\left.\mathrm{UV}, \mathrm{KMnO}_{4}\right)$

m.p.: $55-57^{\circ} \mathrm{C}$

$[\alpha]_{D}^{20.0}=-82.1\left(c=1.0, \mathrm{CHCl}_{3}\right)$

${ }^{1}$ H NMR (600 MHz, C6D6): $\delta 8.66(\mathrm{~s}, 1 \mathrm{H}), 6.70-6.51(\mathrm{~m}, 1 \mathrm{H}), 6.12$ (brs, 1H), $5.00(\mathrm{~d}, J=8.2$ $\mathrm{Hz}, 1 \mathrm{H}), 4.63-4.51(\mathrm{~m}, 1 \mathrm{H}), 4.12-4.07(\mathrm{~m}, 1 \mathrm{H}), 4.03(\mathrm{ddd}, J=8.1,2.2,1.0 \mathrm{~Hz}, 1 \mathrm{H}), 3.24(\mathrm{~s}$, $3 \mathrm{H}), 1.59-1.44(\mathrm{~m}, 8 \mathrm{H}), 1.23(\mathrm{~s}, 9 \mathrm{H}), 1.19-1.11(\mathrm{~m}, 2 \mathrm{H})$.

${ }^{13}$ C NMR (150 MHz, C6D6): $\delta$ 166.7, 158.0, 139.4, 129.7, 110.8, 87.3, 82.2, 75.6, 72.4, 70.2, 51.7, 38.0, 36.0, 28.0, 25.3, 24.2, 24.0.

HRMS (m/z): calculated for $\mathrm{C}_{19} \mathrm{H}_{29} \mathrm{NNaO}_{8}[\mathrm{M}+\mathrm{Na}]^{+}$: 422.1785 , found 422.1790 . 


\section{X-ray Structure 17:}

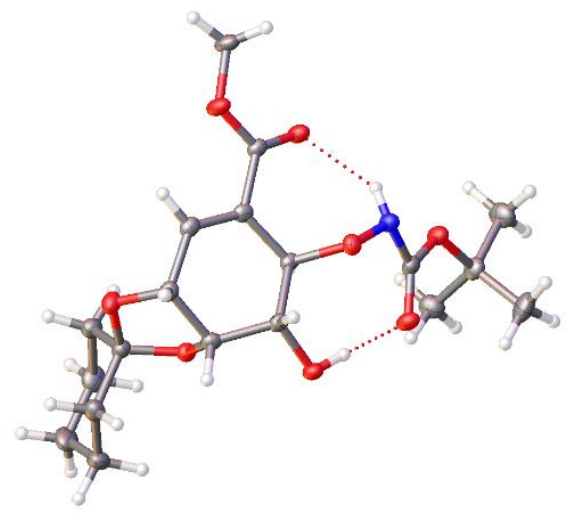

\section{Experimental Summary}

The single crystal X-ray diffraction studies were carried out on a Bruker Smart APEX II CCD diffractometer equipped with $\mathrm{Cu} \mathrm{K}_{\alpha}$ radiation $(\lambda=1.54178 \AA$ ).

Crystals of the subject compound were used as received (grown from ether/hexanes). A $0.3 \times 0.3 \times$ $0.12 \mathrm{~mm}$ piece of a colorless crystal was mounted on a Cryoloop with Paratone oil. Data were collected in a nitrogen gas stream at $100(2) \mathrm{K}$ using $\phi$ and $\varpi$ scans. Crystal-to-detector distance was $40 \mathrm{~mm}$ and exposure time was $1,2,3$, or 5 seconds depending on the $2 \theta$ range per frame using a scan width of $1.25^{\circ}$. Data collection was $99.8 \%$ complete to $66.687^{\circ}$ in $\theta$. A total of 48859 reflections were collected covering the indices, $-10<=\mathrm{h}<=10,-22<=\mathrm{k}<=22-15<=1<=15$. 9879 reflections were found to be symmetry independent. Indexing and unit cell refinement indicated a Primitive, Monoclinic lattice. The space group was found to be $\boldsymbol{P 2}$. The data were integrated using the Bruker SAINT Software program and scaled using the SADABS software program. Solution by direct methods (SHELXT) produced a complete phasing model consistent with the proposed structure.

All nonhydrogen atoms were refined anisotropically by full-matrix least-squares (SHELXL-2014). All carbon bonded hydrogen atoms were placed using a riding model. Their positions were constrained relative to their parent atom using the appropriate HFIX command in SHELXL-2014. Crystallographic data are summarized in Table 1.

Notes: Great data! Absolute stereochemistry was conclusively assigned (Flack $=0.04(3)$ ). There is a minor twin component ( 7\%) and the HKL5 file was used for structure refinement.

Table 12. Crystal data and structure refinement for Baran740.

\section{Report date}

Identification code

Empirical formula

Molecular formula

Formula weight

Temperature

Wavelength

Crystal system

Space group

Unit cell dimensions

Volume

Z

Density (calculated)
2019-09-20

baran740_0m_4_a

C19 H29 N O8

C19 H29 N O8

399.43

$100.0 \mathrm{~K}$

$1.54178 \AA$

Monoclinic

P 1211

$\mathrm{a}=8.5167(2) \AA$

$\mathrm{b}=18.5743(4) \AA$

$\mathrm{c}=13.0021(2) \AA$

1985.22(7) $\AA^{3}$

4

$1.336 \mathrm{Mg} / \mathrm{m}^{3}$ $\alpha=90^{\circ}$.

$\beta=105.1630(10)^{\circ}$.

$\gamma=90^{\circ}$. 


$\begin{array}{ll}\text { Absorption coefficient } & 0.873 \mathrm{~mm}^{-1} \\ \mathrm{~F}(000) & 856 \\ \text { Crystal size } & 0.3 \times 0.3 \times 0.12 \mathrm{~mm}^{3} \\ \text { Crystal color, habit } & \text { colourless block } \\ \text { Theta range for data collection } & 3.522 \text { to } 66.687^{\circ} . \\ \text { Index ranges } & -10<=\mathrm{h}<=10,-22<=\mathrm{k}<=22,-15<=1<=15 \\ \text { Reflections collected } & 48859 \\ \text { Independent reflections } & 9879[\mathrm{R}(\mathrm{int})=?] \\ \text { Completeness to theta }=66.687^{\circ} & 99.8 \% \\ \text { Absorption correction } & \text { Semi-empirical from equivalents } \\ \text { Max. and min. transmission } & 0.752819 \text { and } 0.670175 \\ \text { Refinement method } & \mathrm{Full}-\mathrm{matrix} \text { least-squares on } \mathrm{F}^{2} \\ \text { Data / restraints / parameters } & 6979 / 1 / 527 \\ \text { Goodness-of-fit on F } & 1.053 \\ \text { Final R indices }[\mathrm{I}>2 \text { sigma(I)] } & \mathrm{R} 1=0.0253, \mathrm{wR} 2=0.0683 \\ \text { R indices (all data) } & \mathrm{R} 1=0.0255, \mathrm{wR} 2=0.0685 \\ \text { Absolute structure parameter } & 0.04(3) \\ \text { Extinction coefficient } & 0.0008(2) \\ \text { Largest diff. peak and hole } & 0.198 \text { and }-0.184 \text { e. } \AA^{-3}\end{array}$

Table 13. Atomic coordinates $\left(\mathrm{x} 10^{4}\right)$ and equivalent isotropic displacement parameters $\left(\AA^{2} \times 10^{3}\right)$ for Baran740. $\mathrm{U}(\mathrm{eq})$ is defined as one third of the trace of the orthogonalized $\mathrm{U}^{\mathrm{ij}}$ tensor.

\begin{tabular}{|c|c|c|c|c|}
\hline & $\mathrm{x}$ & $\mathrm{y}$ & $\mathrm{Z}$ & $\mathrm{U}(\mathrm{eq})$ \\
\hline $\mathrm{O}(1 \mathrm{~A})$ & $-80(2)$ & $3671(1)$ & $2320(1)$ & $24(1)$ \\
\hline $\mathrm{O}(2 \mathrm{~A})$ & $1206(2)$ & $5208(1)$ & $6204(1)$ & $22(1)$ \\
\hline $\mathrm{O}(3 \mathrm{~A})$ & $-1105(2)$ & $5341(1)$ & $4926(1)$ & $25(1)$ \\
\hline $\mathrm{O}(4 \mathrm{~A})$ & $1662(2)$ & $3672(1)$ & $6611(1)$ & $19(1)$ \\
\hline $\mathrm{O}(5 \mathrm{~A})$ & $4816(2)$ & $3246(1)$ & $7305(1)$ & $23(1)$ \\
\hline $\mathrm{O}(6 \mathrm{~A})$ & $5336(2)$ & $4312(1)$ & $8198(1)$ & $22(1)$ \\
\hline $\mathrm{O}(7 \mathrm{~A})$ & $2965(2)$ & $2606(1)$ & $5451(1)$ & 21(1) \\
\hline $\mathrm{O}(8 \mathrm{~A})$ & $2336(2)$ & $3306(1)$ & $3422(1)$ & $19(1)$ \\
\hline $\mathrm{N}(1 \mathrm{~A})$ & $2819(2)$ & $4063(1)$ & $7363(1)$ & $21(1)$ \\
\hline $\mathrm{C}(1 \mathrm{~A})$ & $1496(3)$ & 2811(1) & $1620(2)$ & $27(1)$ \\
\hline $\mathrm{C}(2 \mathrm{~A})$ & $3172(4)$ & $2600(1)$ & $1521(2)$ & $35(1)$ \\
\hline $\mathrm{C}(3 \mathrm{~A})$ & $3987(3)$ & $3233(2)$ & $1115(2)$ & $38(1)$ \\
\hline $\mathrm{C}(4 \mathrm{~A})$ & $4083(3)$ & 3881(1) & $1835(2)$ & $32(1)$ \\
\hline $\mathrm{C}(5 \mathrm{~A})$ & $2408(3)$ & $4094(1)$ & $1972(2)$ & $24(1)$ \\
\hline$C(6 A)$ & $1557(3)$ & $3465(1)$ & $2336(2)$ & $20(1)$ \\
\hline $\mathrm{C}(7 \mathrm{~A})$ & $-377(2)$ & $3457(1)$ & $3316(2)$ & $19(1)$ \\
\hline $\mathrm{C}(8 \mathrm{~A})$ & $-524(2)$ & $4122(1)$ & $3938(2)$ & $18(1)$ \\
\hline C(9A) & $389(2)$ & $4274(1)$ & $4910(2)$ & $17(1)$ \\
\hline$C(10 A)$ & $222(2)$ & $4977(1)$ & $5426(2)$ & $18(1)$ \\
\hline $\mathrm{C}(11 \mathrm{~A})$ & $-1265(3)$ & $6054(1)$ & $5343(2)$ & $27(1)$ \\
\hline $\mathrm{C}(12 \mathrm{~A})$ & $1726(2)$ & $3777(1)$ & $5506(2)$ & $17(1)$ \\
\hline$C(13 A)$ & 4391(3) & $3821(1)$ & $7587(2)$ & $19(1)$ \\
\hline $\mathrm{C}(14 \mathrm{~A})$ & $7075(3)$ & $4169(1)$ & $8712(2)$ & $24(1)$ \\
\hline$C(15 A)$ & $7547(3)$ & $4827(2)$ & $9426(2)$ & $34(1)$ \\
\hline$C(16 A)$ & $7250(3)$ & $3483(2)$ & $9365(2)$ & $38(1)$ \\
\hline $\mathrm{C}(17 \mathrm{~A})$ & $8013(3)$ & $4144(1)$ & $7872(2)$ & $30(1)$ \\
\hline $\mathrm{C}(18 \mathrm{~A})$ & $1537(2)$ & $3018(1)$ & $5050(2)$ & $18(1)$ \\
\hline$C(19 A)$ & $1095(3)$ & 2998(1) & $3842(2)$ & $19(1)$ \\
\hline $\mathrm{O}(1 \mathrm{~B})$ & $2141(2)$ & $6416(1)$ & 2061(1) & $24(1)$ \\
\hline $\mathrm{O}(2 \mathrm{~B})$ & $4970(2)$ & $4867(1)$ & $5881(1)$ & $24(1)$ \\
\hline $\mathrm{O}(3 \mathrm{~B})$ & $5451(2)$ & $4640(1)$ & $4307(1)$ & $22(1)$ \\
\hline
\end{tabular}




\begin{tabular}{lrrrr}
$\mathrm{O}(4 \mathrm{~B})$ & $5004(2)$ & $6393(1)$ & $6267(1)$ & $18(1)$ \\
$\mathrm{O}(5 \mathrm{~B})$ & $2918(2)$ & $6859(1)$ & $7382(1)$ & $22(1)$ \\
$\mathrm{O}(6 \mathrm{~B})$ & $3502(2)$ & $5836(1)$ & $8379(1)$ & $22(1)$ \\
$\mathrm{O}(7 \mathrm{~B})$ & $2386(2)$ & $7412(1)$ & $5377(1)$ & $19(1)$ \\
$\mathrm{O}(8 \mathrm{~B})$ & $977(2)$ & $6650(1)$ & $3425(1)$ & $18(1)$ \\
$\mathrm{N}(1 \mathrm{~B})$ & $4784(2)$ & $6025(1)$ & $7155(1)$ & $18(1)$ \\
$\mathrm{C}(1 \mathrm{~B})$ & $-275(3)$ & $5757(1)$ & $2141(2)$ & $24(1)$ \\
$\mathrm{C}(2 \mathrm{~B})$ & $-2011(3)$ & $5805(1)$ & $2248(2)$ & $28(1)$ \\
$\mathrm{C}(3 \mathrm{~B})$ & $-2967(3)$ & $6392(1)$ & $1531(2)$ & $29(1)$ \\
$\mathrm{C}(4 \mathrm{~B})$ & $-2111(3)$ & $7116(1)$ & $1774(2)$ & $30(1)$ \\
$\mathrm{C}(5 \mathrm{~B})$ & $-385(3)$ & $7073(1)$ & $1635(2)$ & $26(1)$ \\
$\mathrm{C}(6 \mathrm{~B})$ & $602(3)$ & $6478(1)$ & $2323(2)$ & $20(1)$ \\
$\mathrm{C}(7 \mathrm{~B})$ & $3404(3)$ & $6614(1)$ & $2982(2)$ & $19(1)$ \\
$\mathrm{C}(8 \mathrm{~B})$ & $4191(2)$ & $5941(1)$ & $3520(2)$ & $18(1)$ \\
$\mathrm{C}(9 \mathrm{~B})$ & $4326(2)$ & $5776(1)$ & $4540(2)$ & $16(1)$ \\
$\mathrm{C}(10 \mathrm{~B})$ & $4960(2)$ & $5060(1)$ & $4984(2)$ & $17(1)$ \\
$\mathrm{C}(11 \mathrm{~B})$ & $6040(3)$ & $3939(1)$ & $4726(2)$ & $24(1)$ \\
$\mathrm{C}(12 \mathrm{~B})$ & $3724(2)$ & $6269(1)$ & $5280(2)$ & $16(1)$ \\
$\mathrm{C}(13 \mathrm{~B})$ & $3638(3)$ & $6292(1)$ & $7613(2)$ & $18(1)$ \\
$\mathrm{C}(14 \mathrm{~B})$ & $2460(3)$ & $6013(1)$ & $9095(2)$ & $22(1)$ \\
$\mathrm{C}(15 \mathrm{~B})$ & $692(3)$ & $5967(1)$ & $8472(2)$ & $30(1)$ \\
$\mathrm{C}(16 \mathrm{~B})$ & $2891(3)$ & $6748(1)$ & $9606(2)$ & $23(1)$ \\
$\mathrm{C}(17 \mathrm{~B})$ & $2907(3)$ & $5415(1)$ & $9921(2)$ & $31(1)$ \\
$\mathrm{C}(18 \mathrm{~B})$ & $3349(2)$ & $7024(1)$ & $4827(2)$ & $17(1)$ \\
$\mathrm{C}(19 \mathrm{~B})$ & $2490(2)$ & $7029(1)$ & $3651(2)$ & $18(1)$ \\
& & & & \\
\hline
\end{tabular}

Table 14. Bond lengths $[\AA]$ and angles $\left[{ }^{\circ}\right]$ for Baran 740 .

\begin{tabular}{llll}
\hline $\mathrm{O}(1 \mathrm{~A})-\mathrm{C}(6 \mathrm{~A})$ & $1.440(3)$ & $\mathrm{C}(4 \mathrm{~A})-\mathrm{H}(4 \mathrm{AA})$ & 0.9900 \\
$\mathrm{O}(1 \mathrm{~A})-\mathrm{C}(7 \mathrm{~A})$ & $1.438(3)$ & $\mathrm{C}(4 \mathrm{~A})-\mathrm{H}(4 \mathrm{AB})$ & 0.9900 \\
$\mathrm{O}(2 \mathrm{~A})-\mathrm{C}(10 \mathrm{~A})$ & $1.211(3)$ & $\mathrm{C}(4 \mathrm{~A})-\mathrm{C}(5 \mathrm{~A})$ & $1.535(3)$ \\
$\mathrm{O}(3 \mathrm{~A})-\mathrm{C}(10 \mathrm{~A})$ & $1.332(3)$ & $\mathrm{C}(5 \mathrm{~A})-\mathrm{H}(5 \mathrm{AA})$ & 0.9900 \\
$\mathrm{O}(3 \mathrm{~A})-\mathrm{C}(11 \mathrm{~A})$ & $1.451(3)$ & $\mathrm{C}(5 \mathrm{~A})-\mathrm{H}(5 \mathrm{AB})$ & 1.5900 \\
$\mathrm{O}(4 \mathrm{~A})-\mathrm{N}(1 \mathrm{~A})$ & $1.397(2)$ & $\mathrm{C}(5 \mathrm{~A})-\mathrm{C}(6 \mathrm{~A})$ & 1.0000 \\
$\mathrm{O}(4 \mathrm{~A})-\mathrm{C}(12 \mathrm{~A})$ & $1.466(2)$ & $\mathrm{C}(7 \mathrm{~A})-\mathrm{H}(7 \mathrm{AA})$ & $1.500(3)$ \\
$\mathrm{O}(5 \mathrm{~A})-\mathrm{C}(13 \mathrm{~A})$ & $1.215(3)$ & $\mathrm{C}(7 \mathrm{~A})-\mathrm{C}(8 \mathrm{~A})$ & $1.523(3)$ \\
$\mathrm{O}(6 \mathrm{~A})-\mathrm{C}(13 \mathrm{~A})$ & $1.333(3)$ & $\mathrm{C}(7 \mathrm{~A})-\mathrm{C}(19 \mathrm{~A})$ & 0.9500 \\
$\mathrm{O}(6 \mathrm{~A})-\mathrm{C}(14 \mathrm{~A})$ & $1.481(2)$ & $\mathrm{C}(8 \mathrm{~A})-\mathrm{H}(8 \mathrm{~A})$ & $1.329(3)$ \\
$\mathrm{O}(7 \mathrm{~A})-\mathrm{H}(7 \mathrm{~A})$ & $\mathrm{C}(8 \mathrm{~A})-\mathrm{C}(9 \mathrm{~A})$ & $1.493(3)$ \\
$\mathrm{O}(7 \mathrm{~A})-\mathrm{C}(18 \mathrm{~A})$ & $0.84(3)$ & $\mathrm{C}(9 \mathrm{~A})-\mathrm{C}(10 \mathrm{~A})$ & $0.911(3)$ \\
$\mathrm{O}(8 \mathrm{~A})-\mathrm{C}(6 \mathrm{~A})$ & $1.415(3)$ & $\mathrm{C}(9 \mathrm{~A})-\mathrm{C}(12 \mathrm{~A})$ & 0.9800 \\
$\mathrm{O}(8 \mathrm{~A})-\mathrm{C}(19 \mathrm{~A})$ & $1.426(2)$ & $\mathrm{C}(11 \mathrm{~A})-\mathrm{H}(11 \mathrm{~A})$ & 0.9800 \\
$\mathrm{~N}(1 \mathrm{~A})-\mathrm{H}(1 \mathrm{~A})$ & $1.431(2)$ & $\mathrm{C}(11 \mathrm{~A})-\mathrm{H}(11 \mathrm{~B})$ & 1.0000 \\
$\mathrm{~N}(1 \mathrm{~A})-\mathrm{C}(13 \mathrm{~A})$ & $0.86(3)$ & $\mathrm{C}(11 \mathrm{~A})-\mathrm{H}(11 \mathrm{C})-\mathrm{H}(12 \mathrm{~A})$ & $1.523(3)$ \\
$\mathrm{C}(1 \mathrm{~A})-\mathrm{H}(1 \mathrm{AA})$ & $1.369(3)$ & $\mathrm{C}(12 \mathrm{~A})-\mathrm{C}(18 \mathrm{~A})$ & $1.523(3)$ \\
$\mathrm{C}(1 \mathrm{~A})-\mathrm{H}(1 \mathrm{AB})$ & 0.9900 & $\mathrm{C}(14 \mathrm{~A})-\mathrm{C}(15 \mathrm{~A})$ & $1.513(3)$ \\
$\mathrm{C}(1 \mathrm{~A})-\mathrm{C}(2 \mathrm{~A})$ & 0.9900 & $\mathrm{C}(14 \mathrm{~A})-\mathrm{C}(16 \mathrm{~A})$ & 0.9800 \\
$\mathrm{C}(1 \mathrm{~A})-\mathrm{C}(6 \mathrm{~A})$ & $1.518(4)$ & $\mathrm{C}(14 \mathrm{~A})-\mathrm{C}(17 \mathrm{~A})$ & 0.9800 \\
$\mathrm{C}(2 \mathrm{~A})-\mathrm{H}(2 \mathrm{AA})$ & $1.523(3)$ & $\mathrm{C}(15 \mathrm{~A})-\mathrm{H}(15 \mathrm{~A})$ & 0.9800 \\
$\mathrm{C}(2 \mathrm{~A})-\mathrm{H}(2 \mathrm{AB})$ & 0.9900 & $\mathrm{C}(15 \mathrm{~A})-\mathrm{H}(15 \mathrm{~B})$ & 0.9800 \\
$\mathrm{C}(2 \mathrm{~A})-\mathrm{C}(3 \mathrm{~A})$ & $\mathrm{C}(15 \mathrm{~A})-\mathrm{H}(15 \mathrm{C})$ & 0.9800 \\
$\mathrm{C}(3 \mathrm{~A})-\mathrm{H}(3 \mathrm{AA})$ & 1.9900 & $\mathrm{C}(16 \mathrm{~A})-\mathrm{H}(16 \mathrm{~A})$ & \\
$\mathrm{C}(3 \mathrm{~A})-\mathrm{H}(3 \mathrm{AB})$ & $\mathrm{C}(16 \mathrm{~A})-\mathrm{H}(16 \mathrm{~B})$ & &
\end{tabular}




\begin{tabular}{|c|c|}
\hline $\mathrm{C}(16 \mathrm{~A})-\mathrm{H}(16 \mathrm{C})$ & 0.9800 \\
\hline $\mathrm{C}(17 \mathrm{~A})-\mathrm{H}(17 \mathrm{~A})$ & 0.9800 \\
\hline $\mathrm{C}(17 \mathrm{~A})-\mathrm{H}(17 \mathrm{~B})$ & 0.9800 \\
\hline $\mathrm{C}(17 \mathrm{~A})-\mathrm{H}(17 \mathrm{C})$ & 0.9800 \\
\hline $\mathrm{C}(18 \mathrm{~A})-\mathrm{H}(18 \mathrm{~A})$ & 1.0000 \\
\hline $\mathrm{C}(18 \mathrm{~A})-\mathrm{C}(19 \mathrm{~A})$ & $1.516(3)$ \\
\hline $\mathrm{C}(19 \mathrm{~A})-\mathrm{H}(19 \mathrm{~A})$ & 1.0000 \\
\hline $\mathrm{O}(1 \mathrm{~B})-\mathrm{C}(6 \mathrm{~B})$ & $1.443(3)$ \\
\hline $\mathrm{O}(1 \mathrm{~B})-\mathrm{C}(7 \mathrm{~B})$ & $1.432(3)$ \\
\hline $\mathrm{O}(2 \mathrm{~B})-\mathrm{C}(10 \mathrm{~B})$ & $1.218(3)$ \\
\hline $\mathrm{O}(3 \mathrm{~B})-\mathrm{C}(10 \mathrm{~B})$ & $1.323(3)$ \\
\hline $\mathrm{O}(3 \mathrm{~B})-\mathrm{C}(11 \mathrm{~B})$ & $1.449(3)$ \\
\hline $\mathrm{O}(4 \mathrm{~B})-\mathrm{N}(1 \mathrm{~B})$ & $1.397(2)$ \\
\hline $\mathrm{O}(4 \mathrm{~B})-\mathrm{C}(12 \mathrm{~B})$ & $1.467(2)$ \\
\hline $\mathrm{O}(5 \mathrm{~B})-\mathrm{C}(13 \mathrm{~B})$ & $1.217(3)$ \\
\hline $\mathrm{O}(6 \mathrm{~B})-\mathrm{C}(13 \mathrm{~B})$ & $1.335(3)$ \\
\hline $\mathrm{O}(6 \mathrm{~B})-\mathrm{C}(14 \mathrm{~B})$ & $1.481(2)$ \\
\hline $\mathrm{O}(7 \mathrm{~B})-\mathrm{H}(7 \mathrm{~B})$ & $0.79(3)$ \\
\hline $\mathrm{O}(7 \mathrm{~B})-\mathrm{C}(18 \mathrm{~B})$ & $1.417(2)$ \\
\hline $\mathrm{O}(8 \mathrm{~B})-\mathrm{C}(6 \mathrm{~B})$ & $1.421(2)$ \\
\hline $\mathrm{O}(8 \mathrm{~B})-\mathrm{C}(19 \mathrm{~B})$ & $1.430(3)$ \\
\hline $\mathrm{N}(1 \mathrm{~B})-\mathrm{H}(1 \mathrm{~B})$ & $0.86(3)$ \\
\hline N(1B)-C(13B) & $1.362(3)$ \\
\hline $\mathrm{C}(1 \mathrm{~B})-\mathrm{H}(1 \mathrm{BA})$ & 0.9900 \\
\hline $\mathrm{C}(1 \mathrm{~B})-\mathrm{H}(1 \mathrm{BB})$ & 0.9900 \\
\hline C(1B)-C(2B) & $1.524(3)$ \\
\hline $\mathrm{C}(1 \mathrm{~B})-\mathrm{C}(6 \mathrm{~B})$ & $1.522(3)$ \\
\hline $\mathrm{C}(2 \mathrm{~B})-\mathrm{H}(2 \mathrm{BA})$ & 0.9900 \\
\hline $\mathrm{C}(2 \mathrm{~B})-\mathrm{H}(2 \mathrm{BB})$ & 0.9900 \\
\hline $\mathrm{C}(2 \mathrm{~B})-\mathrm{C}(3 \mathrm{~B})$ & $1.524(3)$ \\
\hline $\mathrm{C}(3 \mathrm{~B})-\mathrm{H}(3 \mathrm{BA})$ & 0.9900 \\
\hline $\mathrm{C}(3 \mathrm{~B})-\mathrm{H}(3 \mathrm{BB})$ & 0.9900 \\
\hline $\mathrm{C}(3 \mathrm{~B})-\mathrm{C}(4 \mathrm{~B})$ & $1.522(4)$ \\
\hline $\mathrm{C}(4 \mathrm{~B})-\mathrm{H}(4 \mathrm{BA})$ & 0.9900 \\
\hline $\mathrm{C}(4 \mathrm{~B})-\mathrm{H}(4 \mathrm{BB})$ & 0.9900 \\
\hline $\mathrm{C}(4 \mathrm{~B})-\mathrm{C}(5 \mathrm{~B})$ & $1.529(3)$ \\
\hline $\mathrm{C}(5 \mathrm{~B})-\mathrm{H}(5 \mathrm{BA})$ & 0.9900 \\
\hline $\mathrm{C}(5 \mathrm{~B})-\mathrm{H}(5 \mathrm{BB})$ & 0.9900 \\
\hline $\mathrm{C}(5 \mathrm{~B})-\mathrm{C}(6 \mathrm{~B})$ & $1.527(3)$ \\
\hline $\mathrm{C}(7 \mathrm{~B})-\mathrm{H}(7 \mathrm{BA})$ & 1.0000 \\
\hline $\mathrm{C}(7 \mathrm{~B})-\mathrm{C}(8 \mathrm{~B})$ & $1.502(3)$ \\
\hline C(7B)-C(19B) & $1.521(3)$ \\
\hline C(8B)-H(8B) & 0.9500 \\
\hline C(8B)-C(9B) & $1.336(3)$ \\
\hline $\mathrm{C}(9 \mathrm{~B})-\mathrm{C}(10 \mathrm{~B})$ & $1.493(3)$ \\
\hline $\mathrm{C}(9 \mathrm{~B})-\mathrm{C}(12 \mathrm{~B})$ & $1.513(3)$ \\
\hline $\mathrm{C}(11 \mathrm{~B})-\mathrm{H}(11 \mathrm{D})$ & 0.9800 \\
\hline $\mathrm{C}(11 \mathrm{~B})-\mathrm{H}(11 \mathrm{E})$ & 0.9800 \\
\hline $\mathrm{C}(11 \mathrm{~B})-\mathrm{H}(11 \mathrm{~F})$ & 0.9800 \\
\hline $\mathrm{C}(12 \mathrm{~B})-\mathrm{H}(12 \mathrm{~B})$ & 1.0000 \\
\hline $\mathrm{C}(12 \mathrm{~B})-\mathrm{C}(18 \mathrm{~B})$ & $1.522(3)$ \\
\hline$C(14 B)-C(15 B)$ & $1.514(3)$ \\
\hline$C(14 B)-C(16 B)$ & $1.521(3)$ \\
\hline$C(14 B)-C(17 B)$ & $1.522(3)$ \\
\hline $\mathrm{C}(15 \mathrm{~B})-\mathrm{H}(15 \mathrm{D})$ & 0.9800 \\
\hline$C(15 B)-H(15 E)$ & 0.9800 \\
\hline
\end{tabular}

$\begin{array}{ll}\mathrm{C}(15 \mathrm{~B})-\mathrm{H}(15 \mathrm{~F}) & 0.9800 \\ \mathrm{C}(16 \mathrm{~B})-\mathrm{H}(16 \mathrm{D}) & 0.9800 \\ \mathrm{C}(16 \mathrm{~B})-\mathrm{H}(16 \mathrm{E}) & 0.9800 \\ \mathrm{C}(16 \mathrm{~B})-\mathrm{H}(16 \mathrm{~F}) & 0.9800 \\ \mathrm{C}(17 \mathrm{~B})-\mathrm{H}(17 \mathrm{D}) & 0.9800 \\ \mathrm{C}(17 \mathrm{~B})-\mathrm{H}(17 \mathrm{E}) & 0.9800 \\ \mathrm{C}(17 \mathrm{~B})-\mathrm{H}(17 \mathrm{~F}) & 0.9800 \\ \mathrm{C}(18 \mathrm{~B})-\mathrm{H}(18 \mathrm{~B}) & 1.0000 \\ \mathrm{C}(18 \mathrm{~B})-\mathrm{C}(19 \mathrm{~B}) & 1.513(3) \\ \mathrm{C}(19 \mathrm{~B})-\mathrm{H}(19 \mathrm{~B}) & 1.0000\end{array}$

$\mathrm{C}(7 \mathrm{~A})-\mathrm{O}(1 \mathrm{~A})-\mathrm{C}(6 \mathrm{~A}) \quad 108.18(15)$

$\mathrm{C}(10 \mathrm{~A})-\mathrm{O}(3 \mathrm{~A})-\mathrm{C}(11 \mathrm{~A}) \quad 115.57(17)$

$\mathrm{N}(1 \mathrm{~A})-\mathrm{O}(4 \mathrm{~A})-\mathrm{C}(12 \mathrm{~A}) \quad 114.60(14)$

$\mathrm{C}(13 \mathrm{~A})-\mathrm{O}(6 \mathrm{~A})-\mathrm{C}(14 \mathrm{~A}) \quad 121.55(16)$

$\mathrm{C}(18 \mathrm{~A})-\mathrm{O}(7 \mathrm{~A})-\mathrm{H}(7 \mathrm{~A})$

$\mathrm{C}(6 \mathrm{~A})-\mathrm{O}(8 \mathrm{~A})-\mathrm{C}(19 \mathrm{~A}) \quad 105.23(15)$

$\mathrm{O}(4 \mathrm{~A})-\mathrm{N}(1 \mathrm{~A})-\mathrm{H}(1 \mathrm{~A})$

$\mathrm{C}(13 \mathrm{~A})-\mathrm{N}(1 \mathrm{~A})-\mathrm{O}(4 \mathrm{~A})$

$\mathrm{C}(13 \mathrm{~A})-\mathrm{N}(1 \mathrm{~A})-\mathrm{H}(1 \mathrm{~A}) \quad 118.6(19)$

$\mathrm{H}(1 \mathrm{AA})-\mathrm{C}(1 \mathrm{~A})-\mathrm{H}(1 \mathrm{AB})$

$\mathrm{C}(2 \mathrm{~A})-\mathrm{C}(1 \mathrm{~A})-\mathrm{H}(1 \mathrm{AA}) \quad 109.2$

$\mathrm{C}(2 \mathrm{~A})-\mathrm{C}(1 \mathrm{~A})-\mathrm{H}(1 \mathrm{AB}) \quad 109.2$

$\mathrm{C}(2 \mathrm{~A})-\mathrm{C}(1 \mathrm{~A})-\mathrm{C}(6 \mathrm{~A}) \quad 112.1(2)$

$\mathrm{C}(6 \mathrm{~A})-\mathrm{C}(1 \mathrm{~A})-\mathrm{H}(1 \mathrm{AA}) \quad 109.2$

$\mathrm{C}(6 \mathrm{~A})-\mathrm{C}(1 \mathrm{~A})-\mathrm{H}(1 \mathrm{AB}) \quad 109.2$

$\mathrm{C}(1 \mathrm{~A})-\mathrm{C}(2 \mathrm{~A})-\mathrm{H}(2 \mathrm{AA}) \quad 109.5$

$\mathrm{C}(1 \mathrm{~A})-\mathrm{C}(2 \mathrm{~A})-\mathrm{H}(2 \mathrm{AB}) \quad 109.5$

$\mathrm{C}(1 \mathrm{~A})-\mathrm{C}(2 \mathrm{~A})-\mathrm{C}(3 \mathrm{~A}) \quad 110.7(2)$

$\mathrm{H}(2 \mathrm{AA})-\mathrm{C}(2 \mathrm{~A})-\mathrm{H}(2 \mathrm{AB})$

$\mathrm{C}(3 \mathrm{~A})-\mathrm{C}(2 \mathrm{~A})-\mathrm{H}(2 \mathrm{AA}) \quad 109.5$

$\mathrm{C}(3 \mathrm{~A})-\mathrm{C}(2 \mathrm{~A})-\mathrm{H}(2 \mathrm{AB}) \quad 109.5$

$\mathrm{C}(2 \mathrm{~A})-\mathrm{C}(3 \mathrm{~A})-\mathrm{H}(3 \mathrm{AA}) \quad 109.5$

$\mathrm{C}(2 \mathrm{~A})-\mathrm{C}(3 \mathrm{~A})-\mathrm{H}(3 \mathrm{AB}) \quad 109.5$

$\mathrm{H}(3 \mathrm{AA})-\mathrm{C}(3 \mathrm{~A})-\mathrm{H}(3 \mathrm{AB})$

$\mathrm{C}(4 \mathrm{~A})-\mathrm{C}(3 \mathrm{~A})-\mathrm{C}(2 \mathrm{~A}) \quad 110.71(19)$

$\mathrm{C}(4 \mathrm{~A})-\mathrm{C}(3 \mathrm{~A})-\mathrm{H}(3 \mathrm{AA}) \quad 109.5$

$\mathrm{C}(4 \mathrm{~A})-\mathrm{C}(3 \mathrm{~A})-\mathrm{H}(3 \mathrm{AB}) \quad 109.5$

$\mathrm{C}(3 \mathrm{~A})-\mathrm{C}(4 \mathrm{~A})-\mathrm{H}(4 \mathrm{AA}) \quad 109.2$

$\mathrm{C}(3 \mathrm{~A})-\mathrm{C}(4 \mathrm{~A})-\mathrm{H}(4 \mathrm{AB}) \quad 109.2$

$\mathrm{C}(3 \mathrm{~A})-\mathrm{C}(4 \mathrm{~A})-\mathrm{C}(5 \mathrm{~A}) \quad 112.1(2)$

$\mathrm{H}(4 \mathrm{AA})-\mathrm{C}(4 \mathrm{~A})-\mathrm{H}(4 \mathrm{AB})$

$\mathrm{C}(5 \mathrm{~A})-\mathrm{C}(4 \mathrm{~A})-\mathrm{H}(4 \mathrm{AA}) \quad 109.2$

$\mathrm{C}(5 \mathrm{~A})-\mathrm{C}(4 \mathrm{~A})-\mathrm{H}(4 \mathrm{AB}) \quad 109.2$

$\mathrm{C}(4 \mathrm{~A})-\mathrm{C}(5 \mathrm{~A})-\mathrm{H}(5 \mathrm{AA}) \quad 109.2$

$\mathrm{C}(4 \mathrm{~A})-\mathrm{C}(5 \mathrm{~A})-\mathrm{H}(5 \mathrm{AB}) \quad 109.2$

$\mathrm{H}(5 \mathrm{AA})-\mathrm{C}(5 \mathrm{~A})-\mathrm{H}(5 \mathrm{AB}) \quad 107.9$

$\mathrm{C}(6 \mathrm{~A})-\mathrm{C}(5 \mathrm{~A})-\mathrm{C}(4 \mathrm{~A}) \quad 111.91(19)$

$\mathrm{C}(6 \mathrm{~A})-\mathrm{C}(5 \mathrm{~A})-\mathrm{H}(5 \mathrm{AA}) \quad 109.2$

$\mathrm{C}(6 \mathrm{~A})-\mathrm{C}(5 \mathrm{~A})-\mathrm{H}(5 \mathrm{AB}) \quad 109.2$

$\mathrm{O}(1 \mathrm{~A})-\mathrm{C}(6 \mathrm{~A})-\mathrm{C}(1 \mathrm{~A}) \quad 108.97(18)$

$\mathrm{O}(1 \mathrm{~A})-\mathrm{C}(6 \mathrm{~A})-\mathrm{C}(5 \mathrm{~A}) \quad 109.39(17)$

$\mathrm{O}(8 \mathrm{~A})-\mathrm{C}(6 \mathrm{~A})-\mathrm{O}(1 \mathrm{~A}) \quad 105.44(16)$

$\mathrm{O}(8 \mathrm{~A})-\mathrm{C}(6 \mathrm{~A})-\mathrm{C}(1 \mathrm{~A}) \quad 111.89(17)$

$\mathrm{O}(8 \mathrm{~A})-\mathrm{C}(6 \mathrm{~A})-\mathrm{C}(5 \mathrm{~A}) \quad 109.44(17)$

$\mathrm{C}(5 \mathrm{~A})-\mathrm{C}(6 \mathrm{~A})-\mathrm{C}(1 \mathrm{~A}) \quad 111.51(18)$ 


\begin{tabular}{|c|c|}
\hline $\mathrm{O}(1 \mathrm{~A})-\mathrm{C}(7 \mathrm{~A})-\mathrm{H}(7 \mathrm{AA})$ & 110.3 \\
\hline $\mathrm{O}(1 \mathrm{~A})-\mathrm{C}(7 \mathrm{~A})-\mathrm{C}(8 \mathrm{~A})$ & $108.48(17)$ \\
\hline $\mathrm{O}(1 \mathrm{~A})-\mathrm{C}(7 \mathrm{~A})-\mathrm{C}(19 \mathrm{~A})$ & $103.97(16)$ \\
\hline $\mathrm{C}(8 \mathrm{~A})-\mathrm{C}(7 \mathrm{~A})-\mathrm{H}(7 \mathrm{AA})$ & 110.3 \\
\hline C(8A)-C(7A)-C(19A) & $113.21(17)$ \\
\hline $\mathrm{C}(19 \mathrm{~A})-\mathrm{C}(7 \mathrm{~A})-\mathrm{H}(7 \mathrm{AA})$ & 110.3 \\
\hline $\mathrm{C}(7 \mathrm{~A})-\mathrm{C}(8 \mathrm{~A})-\mathrm{H}(8 \mathrm{~A})$ & 117.4 \\
\hline $\mathrm{C}(9 \mathrm{~A})-\mathrm{C}(8 \mathrm{~A})-\mathrm{C}(7 \mathrm{~A})$ & $125.21(19)$ \\
\hline $\mathrm{C}(9 \mathrm{~A})-\mathrm{C}(8 \mathrm{~A})-\mathrm{H}(8 \mathrm{~A})$ & 117.4 \\
\hline $\mathrm{C}(8 \mathrm{~A})-\mathrm{C}(9 \mathrm{~A})-\mathrm{C}(10 \mathrm{~A})$ & $120.77(18)$ \\
\hline $\mathrm{C}(8 \mathrm{~A})-\mathrm{C}(9 \mathrm{~A})-\mathrm{C}(12 \mathrm{~A})$ & $122.20(19)$ \\
\hline$C(10 A)-C(9 A)-C(12 A)$ & $116.84(17)$ \\
\hline $\mathrm{O}(2 \mathrm{~A})-\mathrm{C}(10 \mathrm{~A})-\mathrm{O}(3 \mathrm{~A})$ & $122.83(19)$ \\
\hline $\mathrm{O}(2 \mathrm{~A})-\mathrm{C}(10 \mathrm{~A})-\mathrm{C}(9 \mathrm{~A})$ & $123.98(18)$ \\
\hline $\mathrm{O}(3 \mathrm{~A})-\mathrm{C}(10 \mathrm{~A})-\mathrm{C}(9 \mathrm{~A})$ & $113.17(17)$ \\
\hline $\mathrm{O}(3 \mathrm{~A})-\mathrm{C}(11 \mathrm{~A})-\mathrm{H}(11 \mathrm{~A})$ & 109.5 \\
\hline $\mathrm{O}(3 \mathrm{~A})-\mathrm{C}(11 \mathrm{~A})-\mathrm{H}(11 \mathrm{~B})$ & 109.5 \\
\hline $\mathrm{O}(3 \mathrm{~A})-\mathrm{C}(11 \mathrm{~A})-\mathrm{H}(11 \mathrm{C})$ & 109.5 \\
\hline $\mathrm{H}(11 \mathrm{~A})-\mathrm{C}(11 \mathrm{~A})-\mathrm{H}(11 \mathrm{~B})$ & 109.5 \\
\hline $\mathrm{H}(11 \mathrm{~A})-\mathrm{C}(11 \mathrm{~A})-\mathrm{H}(11 \mathrm{C})$ & 109.5 \\
\hline $\mathrm{H}(11 \mathrm{~B})-\mathrm{C}(11 \mathrm{~A})-\mathrm{H}(11 \mathrm{C})$ & 109.5 \\
\hline $\mathrm{O}(4 \mathrm{~A})-\mathrm{C}(12 \mathrm{~A})-\mathrm{C}(9 \mathrm{~A})$ & $111.55(16)$ \\
\hline $\mathrm{O}(4 \mathrm{~A})-\mathrm{C}(12 \mathrm{~A})-\mathrm{H}(12 \mathrm{~A})$ & 109.9 \\
\hline $\mathrm{O}(4 \mathrm{~A})-\mathrm{C}(12 \mathrm{~A})-\mathrm{C}(18 \mathrm{~A})$ & $103.32(15)$ \\
\hline $\mathrm{C}(9 \mathrm{~A})-\mathrm{C}(12 \mathrm{~A})-\mathrm{H}(12 \mathrm{~A})$ & 109.9 \\
\hline $\mathrm{C}(9 \mathrm{~A})-\mathrm{C}(12 \mathrm{~A})-\mathrm{C}(18 \mathrm{~A})$ & $112.17(17)$ \\
\hline $\mathrm{C}(18 \mathrm{~A})-\mathrm{C}(12 \mathrm{~A})-\mathrm{H}(12 \mathrm{~A})$ & 109.9 \\
\hline $\mathrm{O}(5 \mathrm{~A})-\mathrm{C}(13 \mathrm{~A})-\mathrm{O}(6 \mathrm{~A})$ & $126.90(19)$ \\
\hline $\mathrm{O}(5 \mathrm{~A})-\mathrm{C}(13 \mathrm{~A})-\mathrm{N}(1 \mathrm{~A})$ & $125.2(2)$ \\
\hline $\mathrm{O}(6 \mathrm{~A})-\mathrm{C}(13 \mathrm{~A})-\mathrm{N}(1 \mathrm{~A})$ & $107.87(17)$ \\
\hline $\mathrm{O}(6 \mathrm{~A})-\mathrm{C}(14 \mathrm{~A})-\mathrm{C}(15 \mathrm{~A})$ & $101.83(18)$ \\
\hline $\mathrm{O}(6 \mathrm{~A})-\mathrm{C}(14 \mathrm{~A})-\mathrm{C}(16 \mathrm{~A})$ & $110.10(19)$ \\
\hline $\mathrm{O}(6 \mathrm{~A})-\mathrm{C}(14 \mathrm{~A})-\mathrm{C}(17 \mathrm{~A})$ & $109.53(17)$ \\
\hline$C(16 A)-C(14 A)-C(15 A)$ & $111.2(2)$ \\
\hline $\mathrm{C}(17 \mathrm{~A})-\mathrm{C}(14 \mathrm{~A})-\mathrm{C}(15 \mathrm{~A})$ & $111.1(2)$ \\
\hline$C(17 A)-C(14 A)-C(16 A)$ & $112.6(2)$ \\
\hline $\mathrm{C}(14 \mathrm{~A})-\mathrm{C}(15 \mathrm{~A})-\mathrm{H}(15 \mathrm{~A})$ & 109.5 \\
\hline $\mathrm{C}(14 \mathrm{~A})-\mathrm{C}(15 \mathrm{~A})-\mathrm{H}(15 \mathrm{~B})$ & 109.5 \\
\hline $\mathrm{C}(14 \mathrm{~A})-\mathrm{C}(15 \mathrm{~A})-\mathrm{H}(15 \mathrm{C})$ & 109.5 \\
\hline $\mathrm{H}(15 \mathrm{~A})-\mathrm{C}(15 \mathrm{~A})-\mathrm{H}(15 \mathrm{~B})$ & 109.5 \\
\hline $\mathrm{H}(15 \mathrm{~A})-\mathrm{C}(15 \mathrm{~A})-\mathrm{H}(15 \mathrm{C})$ & 109.5 \\
\hline $\mathrm{H}(15 \mathrm{~B})-\mathrm{C}(15 \mathrm{~A})-\mathrm{H}(15 \mathrm{C})$ & 109.5 \\
\hline $\mathrm{C}(14 \mathrm{~A})-\mathrm{C}(16 \mathrm{~A})-\mathrm{H}(16 \mathrm{~A})$ & 109.5 \\
\hline $\mathrm{C}(14 \mathrm{~A})-\mathrm{C}(16 \mathrm{~A})-\mathrm{H}(16 \mathrm{~B})$ & 109.5 \\
\hline $\mathrm{C}(14 \mathrm{~A})-\mathrm{C}(16 \mathrm{~A})-\mathrm{H}(16 \mathrm{C})$ & 109.5 \\
\hline $\mathrm{H}(16 \mathrm{~A})-\mathrm{C}(16 \mathrm{~A})-\mathrm{H}(16 \mathrm{~B})$ & 109.5 \\
\hline $\mathrm{H}(16 \mathrm{~A})-\mathrm{C}(16 \mathrm{~A})-\mathrm{H}(16 \mathrm{C})$ & 109.5 \\
\hline $\mathrm{H}(16 \mathrm{~B})-\mathrm{C}(16 \mathrm{~A})-\mathrm{H}(16 \mathrm{C})$ & 109.5 \\
\hline $\mathrm{C}(14 \mathrm{~A})-\mathrm{C}(17 \mathrm{~A})-\mathrm{H}(17 \mathrm{~A})$ & 109.5 \\
\hline $\mathrm{C}(14 \mathrm{~A})-\mathrm{C}(17 \mathrm{~A})-\mathrm{H}(17 \mathrm{~B})$ & 109.5 \\
\hline $\mathrm{C}(14 \mathrm{~A})-\mathrm{C}(17 \mathrm{~A})-\mathrm{H}(17 \mathrm{C})$ & 109.5 \\
\hline $\mathrm{H}(17 \mathrm{~A})-\mathrm{C}(17 \mathrm{~A})-\mathrm{H}(17 \mathrm{~B})$ & 109.5 \\
\hline $\mathrm{H}(17 \mathrm{~A})-\mathrm{C}(17 \mathrm{~A})-\mathrm{H}(17 \mathrm{C})$ & 109.5 \\
\hline $\mathrm{H}(17 \mathrm{~B})-\mathrm{C}(17 \mathrm{~A})-\mathrm{H}(17 \mathrm{C})$ & 109.5 \\
\hline $\mathrm{O}(7 \mathrm{~A})-\mathrm{C}(18 \mathrm{~A})-\mathrm{C}(12 \mathrm{~A})$ & $111.35(16)$ \\
\hline $\mathrm{O}(7 \mathrm{~A})-\mathrm{C}(18 \mathrm{~A})-\mathrm{H}(18 \mathrm{~A})$ & 107.6 \\
\hline
\end{tabular}

\begin{tabular}{|c|c|}
\hline $\mathrm{O}(7 \mathrm{~A})-\mathrm{C}(18 \mathrm{~A})-\mathrm{C}(19 \mathrm{~A})$ & $109.00(17)$ \\
\hline $\mathrm{C}(12 \mathrm{~A})-\mathrm{C}(18 \mathrm{~A})-\mathrm{H}(18 \mathrm{~A})$ & 107.6 \\
\hline $\mathrm{C}(19 \mathrm{~A})-\mathrm{C}(18 \mathrm{~A})-\mathrm{C}(12 \mathrm{~A})$ & $113.47(16)$ \\
\hline $\mathrm{C}(19 \mathrm{~A})-\mathrm{C}(18 \mathrm{~A})-\mathrm{H}(18 \mathrm{~A})$ & 107.6 \\
\hline $\mathrm{O}(8 \mathrm{~A})-\mathrm{C}(19 \mathrm{~A})-\mathrm{C}(7 \mathrm{~A})$ & $101.95(15)$ \\
\hline $\mathrm{O}(8 \mathrm{~A})-\mathrm{C}(19 \mathrm{~A})-\mathrm{C}(18 \mathrm{~A})$ & $112.17(16)$ \\
\hline $\mathrm{O}(8 \mathrm{~A})-\mathrm{C}(19 \mathrm{~A})-\mathrm{H}(19 \mathrm{~A})$ & 109.5 \\
\hline $\mathrm{C}(7 \mathrm{~A})-\mathrm{C}(19 \mathrm{~A})-\mathrm{H}(19 \mathrm{~A})$ & 109.5 \\
\hline $\mathrm{C}(18 \mathrm{~A})-\mathrm{C}(19 \mathrm{~A})-\mathrm{C}(7 \mathrm{~A})$ & $113.83(17)$ \\
\hline $\mathrm{C}(18 \mathrm{~A})-\mathrm{C}(19 \mathrm{~A})-\mathrm{H}(19 \mathrm{~A})$ & 109.5 \\
\hline $\mathrm{C}(7 \mathrm{~B})-\mathrm{O}(1 \mathrm{~B})-\mathrm{C}(6 \mathrm{~B})$ & $108.18(15)$ \\
\hline $\mathrm{C}(10 \mathrm{~B})-\mathrm{O}(3 \mathrm{~B})-\mathrm{C}(11 \mathrm{~B})$ & $114.72(16)$ \\
\hline $\mathrm{N}(1 \mathrm{~B})-\mathrm{O}(4 \mathrm{~B})-\mathrm{C}(12 \mathrm{~B})$ & $114.62(14)$ \\
\hline $\mathrm{C}(13 \mathrm{~B})-\mathrm{O}(6 \mathrm{~B})-\mathrm{C}(14 \mathrm{~B})$ & $120.81(16)$ \\
\hline $\mathrm{C}(18 \mathrm{~B})-\mathrm{O}(7 \mathrm{~B})-\mathrm{H}(7 \mathrm{~B})$ & $107(2)$ \\
\hline $\mathrm{C}(6 \mathrm{~B})-\mathrm{O}(8 \mathrm{~B})-\mathrm{C}(19 \mathrm{~B})$ & $105.70(15)$ \\
\hline $\mathrm{O}(4 \mathrm{~B})-\mathrm{N}(1 \mathrm{~B})-\mathrm{H}(1 \mathrm{~B})$ & $113.0(18)$ \\
\hline $\mathrm{C}(13 \mathrm{~B})-\mathrm{N}(1 \mathrm{~B})-\mathrm{O}(4 \mathrm{~B})$ & $116.74(17)$ \\
\hline $\mathrm{C}(13 \mathrm{~B})-\mathrm{N}(1 \mathrm{~B})-\mathrm{H}(1 \mathrm{~B})$ & 119.3(18) \\
\hline $\mathrm{H}(1 \mathrm{BA})-\mathrm{C}(1 \mathrm{~B})-\mathrm{H}(1 \mathrm{BB})$ & 107.8 \\
\hline $\mathrm{C}(2 \mathrm{~B})-\mathrm{C}(1 \mathrm{~B})-\mathrm{H}(1 \mathrm{BA})$ & 109.1 \\
\hline $\mathrm{C}(2 \mathrm{~B})-\mathrm{C}(1 \mathrm{~B})-\mathrm{H}(1 \mathrm{BB})$ & 109.1 \\
\hline $\mathrm{C}(6 \mathrm{~B})-\mathrm{C}(1 \mathrm{~B})-\mathrm{H}(1 \mathrm{BA})$ & 109.1 \\
\hline $\mathrm{C}(6 \mathrm{~B})-\mathrm{C}(1 \mathrm{~B})-\mathrm{H}(1 \mathrm{BB})$ & 109.1 \\
\hline $\mathrm{C}(6 \mathrm{~B})-\mathrm{C}(1 \mathrm{~B})-\mathrm{C}(2 \mathrm{~B})$ & $112.48(18)$ \\
\hline $\mathrm{C}(1 \mathrm{~B})-\mathrm{C}(2 \mathrm{~B})-\mathrm{H}(2 \mathrm{BA})$ & 109.4 \\
\hline $\mathrm{C}(1 \mathrm{~B})-\mathrm{C}(2 \mathrm{~B})-\mathrm{H}(2 \mathrm{BB})$ & 109.4 \\
\hline $\mathrm{C}(1 \mathrm{~B})-\mathrm{C}(2 \mathrm{~B})-\mathrm{C}(3 \mathrm{~B})$ & $111.20(19)$ \\
\hline $\mathrm{H}(2 \mathrm{BA})-\mathrm{C}(2 \mathrm{~B})-\mathrm{H}(2 \mathrm{BB})$ & 108.0 \\
\hline $\mathrm{C}(3 \mathrm{~B})-\mathrm{C}(2 \mathrm{~B})-\mathrm{H}(2 \mathrm{BA})$ & 109.4 \\
\hline $\mathrm{C}(3 \mathrm{~B})-\mathrm{C}(2 \mathrm{~B})-\mathrm{H}(2 \mathrm{BB})$ & 109.4 \\
\hline $\mathrm{C}(2 \mathrm{~B})-\mathrm{C}(3 \mathrm{~B})-\mathrm{H}(3 \mathrm{BA})$ & 109.5 \\
\hline $\mathrm{C}(2 \mathrm{~B})-\mathrm{C}(3 \mathrm{~B})-\mathrm{H}(3 \mathrm{BB})$ & 109.5 \\
\hline $\mathrm{H}(3 \mathrm{BA})-\mathrm{C}(3 \mathrm{~B})-\mathrm{H}(3 \mathrm{BB})$ & 108.1 \\
\hline $\mathrm{C}(4 \mathrm{~B})-\mathrm{C}(3 \mathrm{~B})-\mathrm{C}(2 \mathrm{~B})$ & $110.79(19)$ \\
\hline $\mathrm{C}(4 \mathrm{~B})-\mathrm{C}(3 \mathrm{~B})-\mathrm{H}(3 \mathrm{BA})$ & 109.5 \\
\hline $\mathrm{C}(4 \mathrm{~B})-\mathrm{C}(3 \mathrm{~B})-\mathrm{H}(3 \mathrm{BB})$ & 109.5 \\
\hline $\mathrm{C}(3 \mathrm{~B})-\mathrm{C}(4 \mathrm{~B})-\mathrm{H}(4 \mathrm{BA})$ & 109.5 \\
\hline $\mathrm{C}(3 \mathrm{~B})-\mathrm{C}(4 \mathrm{~B})-\mathrm{H}(4 \mathrm{BB})$ & 109.5 \\
\hline $\mathrm{C}(3 \mathrm{~B})-\mathrm{C}(4 \mathrm{~B})-\mathrm{C}(5 \mathrm{~B})$ & $110.67(19)$ \\
\hline $\mathrm{H}(4 \mathrm{BA})-\mathrm{C}(4 \mathrm{~B})-\mathrm{H}(4 \mathrm{BB})$ & 108.1 \\
\hline $\mathrm{C}(5 \mathrm{~B})-\mathrm{C}(4 \mathrm{~B})-\mathrm{H}(4 \mathrm{BA})$ & 109.5 \\
\hline $\mathrm{C}(5 \mathrm{~B})-\mathrm{C}(4 \mathrm{~B})-\mathrm{H}(4 \mathrm{BB})$ & 109.5 \\
\hline $\mathrm{C}(4 \mathrm{~B})-\mathrm{C}(5 \mathrm{~B})-\mathrm{H}(5 \mathrm{BA})$ & 109.3 \\
\hline $\mathrm{C}(4 \mathrm{~B})-\mathrm{C}(5 \mathrm{~B})-\mathrm{H}(5 \mathrm{BB})$ & 109.3 \\
\hline $\mathrm{H}(5 \mathrm{BA})-\mathrm{C}(5 \mathrm{~B})-\mathrm{H}(5 \mathrm{BB})$ & 108.0 \\
\hline $\mathrm{C}(6 \mathrm{~B})-\mathrm{C}(5 \mathrm{~B})-\mathrm{C}(4 \mathrm{~B})$ & $111.57(19)$ \\
\hline $\mathrm{C}(6 \mathrm{~B})-\mathrm{C}(5 \mathrm{~B})-\mathrm{H}(5 \mathrm{BA})$ & 109.3 \\
\hline $\mathrm{C}(6 \mathrm{~B})-\mathrm{C}(5 \mathrm{~B})-\mathrm{H}(5 \mathrm{BB})$ & 109.3 \\
\hline $\mathrm{O}(1 \mathrm{~B})-\mathrm{C}(6 \mathrm{~B})-\mathrm{C}(1 \mathrm{~B})$ & $109.34(17)$ \\
\hline $\mathrm{O}(1 \mathrm{~B})-\mathrm{C}(6 \mathrm{~B})-\mathrm{C}(5 \mathrm{~B})$ & $108.37(17)$ \\
\hline $\mathrm{O}(8 \mathrm{~B})-\mathrm{C}(6 \mathrm{~B})-\mathrm{O}(1 \mathrm{~B})$ & $106.13(16)$ \\
\hline $\mathrm{O}(8 \mathrm{~B})-\mathrm{C}(6 \mathrm{~B})-\mathrm{C}(1 \mathrm{~B})$ & $109.05(17)$ \\
\hline $\mathrm{O}(8 \mathrm{~B})-\mathrm{C}(6 \mathrm{~B})-\mathrm{C}(5 \mathrm{~B})$ & $111.85(17)$ \\
\hline$C(1 B)-C(6 B)-C(5 B)$ & $111.91(18)$ \\
\hline $\mathrm{O}(1 \mathrm{~B})-\mathrm{C}(7 \mathrm{~B})-\mathrm{H}(7 \mathrm{BA})$ & 110.7 \\
\hline
\end{tabular}




\begin{tabular}{|c|c|c|c|}
\hline $\mathrm{O}(1 \mathrm{~B})-\mathrm{C}(7 \mathrm{~B})-\mathrm{C}(8 \mathrm{~B})$ & $108.77(17)$ & $\mathrm{C}(15 \mathrm{~B})-\mathrm{C}(14 \mathrm{~B})-\mathrm{C}(17 \mathrm{~B})$ & $111.5(2)$ \\
\hline $\mathrm{O}(1 \mathrm{~B})-\mathrm{C}(7 \mathrm{~B})-\mathrm{C}(19 \mathrm{~B})$ & $102.93(16)$ & $\mathrm{C}(16 \mathrm{~B})-\mathrm{C}(14 \mathrm{~B})-\mathrm{C}(17 \mathrm{~B})$ & $111.08(18)$ \\
\hline $\mathrm{C}(8 \mathrm{~B})-\mathrm{C}(7 \mathrm{~B})-\mathrm{H}(7 \mathrm{BA})$ & 110.7 & $\mathrm{C}(14 \mathrm{~B})-\mathrm{C}(15 \mathrm{~B})-\mathrm{H}(15 \mathrm{D})$ & 109.5 \\
\hline C(8B)-C(7B)-C(19B) & $112.82(16)$ & $\mathrm{C}(14 \mathrm{~B})-\mathrm{C}(15 \mathrm{~B})-\mathrm{H}(15 \mathrm{E})$ & 109.5 \\
\hline $\mathrm{C}(19 \mathrm{~B})-\mathrm{C}(7 \mathrm{~B})-\mathrm{H}(7 \mathrm{BA})$ & 110.7 & $\mathrm{C}(14 \mathrm{~B})-\mathrm{C}(15 \mathrm{~B})-\mathrm{H}(15 \mathrm{~F})$ & 109.5 \\
\hline $\mathrm{C}(7 \mathrm{~B})-\mathrm{C}(8 \mathrm{~B})-\mathrm{H}(8 \mathrm{~B})$ & 117.8 & $\mathrm{H}(15 \mathrm{D})-\mathrm{C}(15 \mathrm{~B})-\mathrm{H}(15 \mathrm{E})$ & 109.5 \\
\hline $\mathrm{C}(9 \mathrm{~B})-\mathrm{C}(8 \mathrm{~B})-\mathrm{C}(7 \mathrm{~B})$ & $124.43(18)$ & $\mathrm{H}(15 \mathrm{D})-\mathrm{C}(15 \mathrm{~B})-\mathrm{H}(15 \mathrm{~F})$ & 109.5 \\
\hline $\mathrm{C}(9 \mathrm{~B})-\mathrm{C}(8 \mathrm{~B})-\mathrm{H}(8 \mathrm{~B})$ & 117.8 & $\mathrm{H}(15 \mathrm{E})-\mathrm{C}(15 \mathrm{~B})-\mathrm{H}(15 \mathrm{~F})$ & 109.5 \\
\hline $\mathrm{C}(8 \mathrm{~B})-\mathrm{C}(9 \mathrm{~B})-\mathrm{C}(10 \mathrm{~B})$ & $121.11(18)$ & $\mathrm{C}(14 \mathrm{~B})-\mathrm{C}(16 \mathrm{~B})-\mathrm{H}(16 \mathrm{D})$ & 109.5 \\
\hline $\mathrm{C}(8 \mathrm{~B})-\mathrm{C}(9 \mathrm{~B})-\mathrm{C}(12 \mathrm{~B})$ & $122.48(18)$ & $\mathrm{C}(14 \mathrm{~B})-\mathrm{C}(16 \mathrm{~B})-\mathrm{H}(16 \mathrm{E})$ & 109.5 \\
\hline$C(10 B)-C(9 B)-C(12 B)$ & $116.22(17)$ & $\mathrm{C}(14 \mathrm{~B})-\mathrm{C}(16 \mathrm{~B})-\mathrm{H}(16 \mathrm{~F})$ & 109.5 \\
\hline $\mathrm{O}(2 \mathrm{~B})-\mathrm{C}(10 \mathrm{~B})-\mathrm{O}(3 \mathrm{~B})$ & $122.62(19)$ & $H(16 D)-C(16 B)-H(16 E)$ & 109.5 \\
\hline $\mathrm{O}(2 \mathrm{~B})-\mathrm{C}(10 \mathrm{~B})-\mathrm{C}(9 \mathrm{~B})$ & $122.97(18)$ & $H(16 D)-C(16 B)-H(16 F)$ & 109.5 \\
\hline $\mathrm{O}(3 \mathrm{~B})-\mathrm{C}(10 \mathrm{~B})-\mathrm{C}(9 \mathrm{~B})$ & $114.36(17)$ & $\mathrm{H}(16 \mathrm{E})-\mathrm{C}(16 \mathrm{~B})-\mathrm{H}(16 \mathrm{~F})$ & 109.5 \\
\hline $\mathrm{O}(3 \mathrm{~B})-\mathrm{C}(11 \mathrm{~B})-\mathrm{H}(11 \mathrm{D})$ & 109.5 & $\mathrm{C}(14 \mathrm{~B})-\mathrm{C}(17 \mathrm{~B})-\mathrm{H}(17 \mathrm{D})$ & 109.5 \\
\hline $\mathrm{O}(3 \mathrm{~B})-\mathrm{C}(11 \mathrm{~B})-\mathrm{H}(11 \mathrm{E})$ & 109.5 & $\mathrm{C}(14 \mathrm{~B})-\mathrm{C}(17 \mathrm{~B})-\mathrm{H}(17 \mathrm{E})$ & 109.5 \\
\hline $\mathrm{O}(3 \mathrm{~B})-\mathrm{C}(11 \mathrm{~B})-\mathrm{H}(11 \mathrm{~F})$ & 109.5 & $\mathrm{C}(14 \mathrm{~B})-\mathrm{C}(17 \mathrm{~B})-\mathrm{H}(17 \mathrm{~F})$ & 109.5 \\
\hline $\mathrm{H}(11 \mathrm{D})-\mathrm{C}(11 \mathrm{~B})-\mathrm{H}(11 \mathrm{E})$ & 109.5 & $\mathrm{H}(17 \mathrm{D})-\mathrm{C}(17 \mathrm{~B})-\mathrm{H}(17 \mathrm{E})$ & 109.5 \\
\hline $\mathrm{H}(11 \mathrm{D})-\mathrm{C}(11 \mathrm{~B})-\mathrm{H}(11 \mathrm{~F})$ & 109.5 & $\mathrm{H}(17 \mathrm{D})-\mathrm{C}(17 \mathrm{~B})-\mathrm{H}(17 \mathrm{~F})$ & 109.5 \\
\hline $\mathrm{H}(11 \mathrm{E})-\mathrm{C}(11 \mathrm{~B})-\mathrm{H}(11 \mathrm{~F})$ & 109.5 & $\mathrm{H}(17 \mathrm{E})-\mathrm{C}(17 \mathrm{~B})-\mathrm{H}(17 \mathrm{~F})$ & 109.5 \\
\hline $\mathrm{O}(4 \mathrm{~B})-\mathrm{C}(12 \mathrm{~B})-\mathrm{C}(9 \mathrm{~B})$ & $110.68(15)$ & $\mathrm{O}(7 \mathrm{~B})-\mathrm{C}(18 \mathrm{~B})-\mathrm{C}(12 \mathrm{~B})$ & $111.29(15)$ \\
\hline $\mathrm{O}(4 \mathrm{~B})-\mathrm{C}(12 \mathrm{~B})-\mathrm{H}(12 \mathrm{~B})$ & 110.1 & $\mathrm{O}(7 \mathrm{~B})-\mathrm{C}(18 \mathrm{~B})-\mathrm{H}(18 \mathrm{~B})$ & 107.9 \\
\hline $\mathrm{O}(4 \mathrm{~B})-\mathrm{C}(12 \mathrm{~B})-\mathrm{C}(18 \mathrm{~B})$ & $103.10(15)$ & $\mathrm{O}(7 \mathrm{~B})-\mathrm{C}(18 \mathrm{~B})-\mathrm{C}(19 \mathrm{~B})$ & $108.47(17)$ \\
\hline $\mathrm{C}(9 \mathrm{~B})-\mathrm{C}(12 \mathrm{~B})-\mathrm{H}(12 \mathrm{~B})$ & 110.1 & $\mathrm{C}(12 \mathrm{~B})-\mathrm{C}(18 \mathrm{~B})-\mathrm{H}(18 \mathrm{~B})$ & 107.9 \\
\hline C(9B)-C(12B)-C(18B) & $112.58(16)$ & $\mathrm{C}(19 \mathrm{~B})-\mathrm{C}(18 \mathrm{~B})-\mathrm{C}(12 \mathrm{~B})$ & $113.16(16)$ \\
\hline $\mathrm{C}(18 \mathrm{~B})-\mathrm{C}(12 \mathrm{~B})-\mathrm{H}(12 \mathrm{~B})$ & 110.1 & $\mathrm{C}(19 \mathrm{~B})-\mathrm{C}(18 \mathrm{~B})-\mathrm{H}(18 \mathrm{~B})$ & 107.9 \\
\hline $\mathrm{O}(5 \mathrm{~B})-\mathrm{C}(13 \mathrm{~B})-\mathrm{O}(6 \mathrm{~B})$ & 126.63(19) & $\mathrm{O}(8 \mathrm{~B})-\mathrm{C}(19 \mathrm{~B})-\mathrm{C}(7 \mathrm{~B})$ & $101.65(16)$ \\
\hline $\mathrm{O}(5 \mathrm{~B})-\mathrm{C}(13 \mathrm{~B})-\mathrm{N}(1 \mathrm{~B})$ & $125.39(19)$ & $\mathrm{O}(8 \mathrm{~B})-\mathrm{C}(19 \mathrm{~B})-\mathrm{C}(18 \mathrm{~B})$ & $112.13(16)$ \\
\hline $\mathrm{O}(6 \mathrm{~B})-\mathrm{C}(13 \mathrm{~B})-\mathrm{N}(1 \mathrm{~B})$ & 107.93(17) & $\mathrm{O}(8 \mathrm{~B})-\mathrm{C}(19 \mathrm{~B})-\mathrm{H}(19 \mathrm{~B})$ & 109.9 \\
\hline $\mathrm{O}(6 \mathrm{~B})-\mathrm{C}(14 \mathrm{~B})-\mathrm{C}(15 \mathrm{~B})$ & $109.04(17)$ & $\mathrm{C}(7 \mathrm{~B})-\mathrm{C}(19 \mathrm{~B})-\mathrm{H}(19 \mathrm{~B})$ & 109.9 \\
\hline $\mathrm{O}(6 \mathrm{~B})-\mathrm{C}(14 \mathrm{~B})-\mathrm{C}(16 \mathrm{~B})$ & $110.96(17)$ & $\mathrm{C}(18 \mathrm{~B})-\mathrm{C}(19 \mathrm{~B})-\mathrm{C}(7 \mathrm{~B})$ & $113.04(17)$ \\
\hline $\mathrm{O}(6 \mathrm{~B})-\mathrm{C}(14 \mathrm{~B})-\mathrm{C}(17 \mathrm{~B})$ & 101.61(17) & $\mathrm{C}(18 \mathrm{~B})-\mathrm{C}(19 \mathrm{~B})-\mathrm{H}(19 \mathrm{~B})$ & 109.9 \\
\hline$C(15 B)-C(14 B)-C(16 B)$ & $112.15(19)$ & & \\
\hline
\end{tabular}

Symmetry transformations used to generate equivalent atoms:

Table 15. Anisotropic displacement parameters $\left(\AA^{2} \times 10^{3}\right)$ for Baran740. The anisotropic displacement factor exponent takes the form: $-2 \pi^{2}\left[h^{2} a^{* 2} U^{11}+\ldots+2 h k a^{*} b^{*} U^{12}\right]$

\begin{tabular}{lcccccc}
\hline & $\mathrm{U}^{11}$ & $\mathrm{U}^{22}$ & $\mathrm{U}^{33}$ & $\mathrm{U}^{23}$ & $\mathrm{U}^{13}$ & $\mathrm{U}^{12}$ \\
\hline $\mathrm{O}(1 \mathrm{~A})$ & $22(1)$ & $29(1)$ & $22(1)$ & $5(1)$ & $6(1)$ & $4(1)$ \\
$\mathrm{O}(2 \mathrm{~A})$ & $20(1)$ & $17(1)$ & $27(1)$ & $-2(1)$ & $1(1)$ & $2(1)$ \\
$\mathrm{O}(3 \mathrm{~A})$ & $22(1)$ & $18(1)$ & $31(1)$ & $-4(1)$ & $-1(1)$ & $4(1)$ \\
$\mathrm{O}(4 \mathrm{~A})$ & $17(1)$ & $21(1)$ & $19(1)$ & $0(1)$ & $4(1)$ & $-3(1)$ \\
$\mathrm{O}(5 \mathrm{~A})$ & $21(1)$ & $16(1)$ & $30(1)$ & $-1(1)$ & $1(1)$ & $2(1)$ \\
$\mathrm{O}(6 \mathrm{~A})$ & $20(1)$ & $20(1)$ & $24(1)$ & $-3(1)$ & $0(1)$ & $2(1)$ \\
$\mathrm{O}(7 \mathrm{~A})$ & $20(1)$ & $17(1)$ & $23(1)$ & $-1(1)$ & $3(1)$ & $4(1)$ \\
$\mathrm{O}(8 \mathrm{~A})$ & $19(1)$ & $19(1)$ & $19(1)$ & $1(1)$ & $6(1)$ & $0(1)$ \\
$\mathrm{N}(1 \mathrm{~A})$ & $20(1)$ & $18(1)$ & $22(1)$ & $-2(1)$ & $2(1)$ & $1(1)$ \\
$\mathrm{C}(1 \mathrm{~A})$ & $36(1)$ & $22(1)$ & $22(1)$ & $-2(1)$ & $5(1)$ & $0(1)$ \\
$\mathrm{C}(2 \mathrm{~A})$ & $50(2)$ & $30(1)$ & $28(1)$ & $-2(1)$ & $13(1)$ & $14(1)$ \\
$\mathrm{C}(3 \mathrm{~A})$ & $42(1)$ & $49(2)$ & $29(1)$ & $4(1)$ & $19(1)$ & $16(1)$ \\
$\mathrm{C}(4 \mathrm{~A})$ & $30(1)$ & $39(1)$ & $31(1)$ & $8(1)$ & $15(1)$ & $-1(1)$ \\
$\mathrm{C}(5 \mathrm{~A})$ & $28(1)$ & $20(1)$ & $25(1)$ & $2(1)$ & $10(1)$ & $2(1)$ \\
& & & & &
\end{tabular}




\begin{tabular}{|c|c|c|c|c|c|c|}
\hline$C(6 \mathrm{~A})$ & $22(1)$ & $18(1)$ & $20(1)$ & $2(1)$ & $4(1)$ & $2(1)$ \\
\hline$C(7 A)$ & $17(1)$ & $18(1)$ & 21(1) & 1(1) & $3(1)$ & $-3(1)$ \\
\hline $\mathrm{C}(8 \mathrm{~A})$ & $14(1)$ & $17(1)$ & $24(1)$ & $4(1)$ & $5(1)$ & $-2(1)$ \\
\hline $\mathrm{C}(9 \mathrm{~A})$ & $16(1)$ & $14(1)$ & $23(1)$ & 1(1) & $7(1)$ & $-3(1)$ \\
\hline $\mathrm{C}(10 \mathrm{~A})$ & $14(1)$ & $16(1)$ & $22(1)$ & $3(1)$ & $5(1)$ & $0(1)$ \\
\hline $\mathrm{C}(11 \mathrm{~A})$ & $24(1)$ & $18(1)$ & $34(1)$ & $-3(1)$ & $2(1)$ & $6(1)$ \\
\hline $\mathrm{C}(12 \mathrm{~A})$ & $15(1)$ & $16(1)$ & $20(1)$ & $2(1)$ & $6(1)$ & $0(1)$ \\
\hline $\mathrm{C}(13 \mathrm{~A})$ & $20(1)$ & $17(1)$ & 19(1) & $4(1)$ & $3(1)$ & 1(1) \\
\hline $\mathrm{C}(14 \mathrm{~A})$ & $18(1)$ & $26(1)$ & $24(1)$ & $0(1)$ & $-2(1)$ & $0(1)$ \\
\hline $\mathrm{C}(15 \mathrm{~A})$ & $27(1)$ & $38(1)$ & $32(1)$ & $-11(1)$ & $-2(1)$ & $-1(1)$ \\
\hline $\mathrm{C}(16 \mathrm{~A})$ & $33(1)$ & $39(1)$ & $34(1)$ & $12(1)$ & $-4(1)$ & $2(1)$ \\
\hline $\mathrm{C}(17 \mathrm{~A})$ & $26(1)$ & $30(1)$ & $33(1)$ & $-6(1)$ & $7(1)$ & $-3(1)$ \\
\hline $\mathrm{C}(18 \mathrm{~A})$ & $16(1)$ & $15(1)$ & $21(1)$ & $2(1)$ & $4(1)$ & $-1(1)$ \\
\hline $\mathrm{C}(19 \mathrm{~A})$ & 19(1) & $14(1)$ & $24(1)$ & $0(1)$ & $6(1)$ & $-2(1)$ \\
\hline $\mathrm{O}(1 \mathrm{~B})$ & $25(1)$ & $34(1)$ & $15(1)$ & $-2(1)$ & $7(1)$ & 1(1) \\
\hline $\mathrm{O}(2 \mathrm{~B})$ & $32(1)$ & $17(1)$ & $25(1)$ & $2(1)$ & $13(1)$ & $4(1)$ \\
\hline $\mathrm{O}(3 \mathrm{~B})$ & $24(1)$ & $18(1)$ & $26(1)$ & $-1(1)$ & $10(1)$ & $4(1)$ \\
\hline $\mathrm{O}(4 \mathrm{~B})$ & 19(1) & $20(1)$ & $14(1)$ & $0(1)$ & $5(1)$ & $-2(1)$ \\
\hline $\mathrm{O}(5 \mathrm{~B})$ & $29(1)$ & 19(1) & 19(1) & $2(1)$ & $9(1)$ & $4(1)$ \\
\hline $\mathrm{O}(6 \mathrm{~B})$ & $30(1)$ & 19(1) & $20(1)$ & $4(1)$ & $12(1)$ & $6(1)$ \\
\hline $\mathrm{O}(7 \mathrm{~B})$ & $25(1)$ & $16(1)$ & $18(1)$ & $-1(1)$ & $7(1)$ & $4(1)$ \\
\hline $\mathrm{O}(8 \mathrm{~B})$ & 19(1) & $22(1)$ & $15(1)$ & $-1(1)$ & $4(1)$ & $-1(1)$ \\
\hline $\mathrm{N}(1 \mathrm{~B})$ & $23(1)$ & $16(1)$ & $16(1)$ & 1(1) & $6(1)$ & $3(1)$ \\
\hline $\mathrm{C}(1 \mathrm{~B})$ & $28(1)$ & $22(1)$ & $20(1)$ & $-2(1)$ & $3(1)$ & $2(1)$ \\
\hline $\mathrm{C}(2 \mathrm{~B})$ & $27(1)$ & $34(1)$ & $23(1)$ & $0(1)$ & $4(1)$ & $-5(1)$ \\
\hline $\mathrm{C}(3 \mathrm{~B})$ & $24(1)$ & $44(1)$ & 19(1) & $-2(1)$ & $4(1)$ & $5(1)$ \\
\hline$C(4 B)$ & $30(1)$ & $33(1)$ & $24(1)$ & $-2(1)$ & $2(1)$ & $13(1)$ \\
\hline $\mathrm{C}(5 \mathrm{~B})$ & $30(1)$ & $25(1)$ & $20(1)$ & $2(1)$ & $3(1)$ & $3(1)$ \\
\hline$C(6 B)$ & $22(1)$ & $24(1)$ & $16(1)$ & $-2(1)$ & $6(1)$ & 1(1) \\
\hline $\mathrm{C}(7 \mathrm{~B})$ & 21(1) & 21(1) & $18(1)$ & 1(1) & $8(1)$ & $-2(1)$ \\
\hline $\mathrm{C}(8 \mathrm{~B})$ & $17(1)$ & $18(1)$ & $21(1)$ & $-4(1)$ & $9(1)$ & $-2(1)$ \\
\hline $\mathrm{C}(9 \mathrm{~B})$ & $14(1)$ & $15(1)$ & 21(1) & $-1(1)$ & $6(1)$ & $-2(1)$ \\
\hline $\mathrm{C}(10 \mathrm{~B})$ & $15(1)$ & $14(1)$ & $23(1)$ & $-3(1)$ & $7(1)$ & $-3(1)$ \\
\hline $\mathrm{C}(11 \mathrm{~B})$ & $22(1)$ & $18(1)$ & $33(1)$ & $0(1)$ & $11(1)$ & $5(1)$ \\
\hline $\mathrm{C}(12 \mathrm{~B})$ & $14(1)$ & $16(1)$ & $17(1)$ & $-1(1)$ & $4(1)$ & $-1(1)$ \\
\hline $\mathrm{C}(13 \mathrm{~B})$ & $22(1)$ & $18(1)$ & $14(1)$ & $-2(1)$ & $3(1)$ & $-2(1)$ \\
\hline$C(14 B)$ & $28(1)$ & 21(1) & 19(1) & 1(1) & $12(1)$ & 1(1) \\
\hline$C(15 B)$ & $29(1)$ & $29(1)$ & $34(1)$ & $-4(1)$ & $13(1)$ & $-4(1)$ \\
\hline$C(16 B)$ & $31(1)$ & $22(1)$ & 19(1) & $0(1)$ & $9(1)$ & $0(1)$ \\
\hline $\mathrm{C}(17 \mathrm{~B})$ & $46(1)$ & $24(1)$ & $28(1)$ & $5(1)$ & $18(1)$ & $2(1)$ \\
\hline$C(18 B)$ & $18(1)$ & $15(1)$ & $18(1)$ & $-1(1)$ & $7(1)$ & $-1(1)$ \\
\hline$C(19 B)$ & $20(1)$ & $15(1)$ & $20(1)$ & $3(1)$ & $8(1)$ & $-1(1)$ \\
\hline
\end{tabular}

Table 16. Hydrogen coordinates (x $\left.10^{4}\right)$ and isotropic displacement parameters $\left(\AA^{2} \times 10^{3}\right)$ for Baran740.

\begin{tabular}{lcccc}
\hline & \multicolumn{1}{c}{$\mathrm{x}$} & \multicolumn{1}{c}{$\mathrm{y}$} & $\mathrm{z}$ & $\mathrm{U}(\mathrm{eq})$ \\
\hline $\mathrm{H}(7 \mathrm{~A})$ & $3420(40)$ & $2786(16)$ & $6050(30)$ & 31 \\
$\mathrm{H}(1 \mathrm{~A})$ & $2640(30)$ & $4516(17)$ & $7290(20)$ & 25 \\
$\mathrm{H}(1 \mathrm{AA})$ & 1013 & 2401 & 1915 & 33 \\
$\mathrm{H}(1 \mathrm{AB})$ & 786 & 2919 & 903 & 33 \\
$\mathrm{H}(2 \mathrm{AA})$ & 3073 & 2189 & 1022 & 42 \\
$\mathrm{H}(2 \mathrm{AB})$ & 3853 & 2444 & 2225 & 42 \\
& & & &
\end{tabular}




\begin{tabular}{|c|c|c|c|c|}
\hline $\mathrm{H}(3 \mathrm{AA})$ & 5098 & 3092 & 1088 & 46 \\
\hline $\mathrm{H}(3 \mathrm{AB})$ & 3359 & 3359 & 383 & 46 \\
\hline $\mathrm{H}(4 \mathrm{AA})$ & 4548 & 4292 & 1530 & 39 \\
\hline $\mathrm{H}(4 \mathrm{AB})$ & 4820 & 3771 & 2542 & 39 \\
\hline $\mathrm{H}(5 \mathrm{AA})$ & 2542 & 4487 & 2501 & 29 \\
\hline $\mathrm{H}(5 \mathrm{AB})$ & 1724 & 4276 & 1285 & 29 \\
\hline $\mathrm{H}(7 \mathrm{AA})$ & -1396 & 3164 & 3186 & 23 \\
\hline $\mathrm{H}(8 \mathrm{~A})$ & -1334 & 4462 & 3612 & 22 \\
\hline $\mathrm{H}(11 \mathrm{~A})$ & -2186 & 6304 & 4866 & 40 \\
\hline $\mathrm{H}(11 \mathrm{~B})$ & -265 & 6328 & 5392 & 40 \\
\hline $\mathrm{H}(11 \mathrm{C})$ & -1453 & 6012 & 6052 & 40 \\
\hline $\mathrm{H}(12 \mathrm{~A})$ & 2810 & 3977 & 5491 & 20 \\
\hline $\mathrm{H}(15 \mathrm{~A})$ & 7295 & 5265 & 8994 & 51 \\
\hline $\mathrm{H}(15 \mathrm{~B})$ & 8715 & 4811 & 9775 & 51 \\
\hline $\mathrm{H}(15 \mathrm{C})$ & 6935 & 4827 & 9968 & 51 \\
\hline $\mathrm{H}(16 \mathrm{~A})$ & 6469 & 3487 & 9800 & 57 \\
\hline $\mathrm{H}(16 \mathrm{~B})$ & 8358 & 3452 & 9831 & 57 \\
\hline $\mathrm{H}(16 \mathrm{C})$ & 7038 & 3066 & 8886 & 57 \\
\hline $\mathrm{H}(17 \mathrm{~A})$ & 7603 & 3747 & 7377 & 45 \\
\hline $\mathrm{H}(17 \mathrm{~B})$ & 9170 & 4067 & 8216 & 45 \\
\hline $\mathrm{H}(17 \mathrm{C})$ & 7873 & 4600 & 7480 & 45 \\
\hline $\mathrm{H}(18 \mathrm{~A})$ & 636 & 2780 & 5284 & 21 \\
\hline $\mathrm{H}(19 \mathrm{~A})$ & 896 & 2488 & 3593 & 22 \\
\hline $\mathrm{H}(7 \mathrm{~B})$ & $2650(40)$ & $7283(16)$ & $5980(30)$ & 29 \\
\hline $\mathrm{H}(1 \mathrm{~B})$ & $4890(30)$ & $5567(17)$ & $7110(20)$ & 22 \\
\hline H(1BA) & 339 & 5403 & 2663 & 29 \\
\hline $\mathrm{H}(1 \mathrm{BB})$ & -298 & 5580 & 1419 & 29 \\
\hline $\mathrm{H}(2 \mathrm{BA})$ & -1988 & 5910 & 2998 & 34 \\
\hline $\mathrm{H}(2 \mathrm{BB})$ & -2563 & 5336 & 2054 & 34 \\
\hline H(3BA) & -3080 & 6265 & 776 & 35 \\
\hline $\mathrm{H}(3 \mathrm{BB})$ & -4071 & 6427 & 1642 & 35 \\
\hline H(4BA) & -2065 & 7259 & 2514 & 36 \\
\hline H(4BB) & -2735 & 7487 & 1288 & 36 \\
\hline H(5BA) & -438 & 6979 & 877 & 31 \\
\hline $\mathrm{H}(5 \mathrm{BB})$ & 169 & 7541 & 1834 & 31 \\
\hline $\mathrm{H}(7 \mathrm{BA})$ & 4225 & 6929 & 2777 & 23 \\
\hline $\mathrm{H}(8 \mathrm{~B})$ & 4622 & 5610 & 3108 & 22 \\
\hline $\mathrm{H}(11 \mathrm{D})$ & 6427 & 3673 & 4189 & 35 \\
\hline $\mathrm{H}(11 \mathrm{E})$ & 5155 & 3671 & 4903 & 35 \\
\hline $\mathrm{H}(11 \mathrm{~F})$ & 6937 & 4000 & 5369 & 35 \\
\hline $\mathrm{H}(12 \mathrm{~B})$ & 2740 & 6058 & 5448 & 19 \\
\hline $\mathrm{H}(15 \mathrm{D})$ & 482 & 6315 & 7885 & 45 \\
\hline $\mathrm{H}(15 \mathrm{E})$ & -5 & 6077 & 8944 & 45 \\
\hline $\mathrm{H}(15 \mathrm{~F})$ & 455 & 5480 & 8184 & 45 \\
\hline H(16D) & 4076 & 6798 & 9844 & 35 \\
\hline $\mathrm{H}(16 \mathrm{E})$ & 2430 & 6796 & 10219 & 35 \\
\hline $\mathrm{H}(16 \mathrm{~F})$ & 2443 & 7125 & 9085 & 35 \\
\hline H(17D) & 2683 & 4947 & 9566 & 47 \\
\hline $\mathrm{H}(17 \mathrm{E})$ & 2261 & 5466 & 10440 & 47 \\
\hline $\mathrm{H}(17 \mathrm{~F})$ & 4066 & 5448 & 10290 & 47 \\
\hline $\mathrm{H}(18 \mathrm{~B})$ & 4404 & 7286 & 4926 & 20 \\
\hline $\mathrm{H}(19 \mathrm{~B})$ & 2306 & 7536 & 3389 & 21 \\
\hline
\end{tabular}

Table 17. Hydrogen bonds for Baran740 [ $\AA^{\mathrm{A}}$ and $\left.{ }^{\circ}\right]$. 


\begin{tabular}{|c|c|c|c|c|}
\hline D-H...A & $d(D-H)$ & $\mathrm{d}(\mathrm{H} \ldots \mathrm{A})$ & $\mathrm{d}(\mathrm{D} \ldots \mathrm{A})$ & $<(\mathrm{DHA})$ \\
\hline $\mathrm{O}(7 \mathrm{~A})-\mathrm{H}(7 \mathrm{~A}) \ldots \mathrm{O}(5 \mathrm{~A})$ & $0.84(3)$ & $1.95(3)$ & $2.779(2)$ & $169(3)$ \\
\hline $\mathrm{N}(1 \mathrm{~A})-\mathrm{H}(1 \mathrm{~A}) \ldots \mathrm{O}(2 \mathrm{~A})$ & $0.86(3)$ & $2.06(3)$ & $2.756(2)$ & $138(3)$ \\
\hline $\mathrm{O}(7 \mathrm{~B})-\mathrm{H}(7 \mathrm{~B}) \ldots \mathrm{O}(5 \mathrm{~B})$ & $0.79(3)$ & $1.94(3)$ & $2.726(2)$ & $169(3)$ \\
\hline $\mathrm{N}(1 \mathrm{~B})-\mathrm{H}(1 \mathrm{~B}) \ldots \mathrm{O}(2 \mathrm{~B})$ & $0.86(3)$ & $2.08(3)$ & $2.745(2)$ & $134(2)$ \\
\hline
\end{tabular}

Symmetry transformations used to generate equivalent atoms:

\section{Compound SI-13}

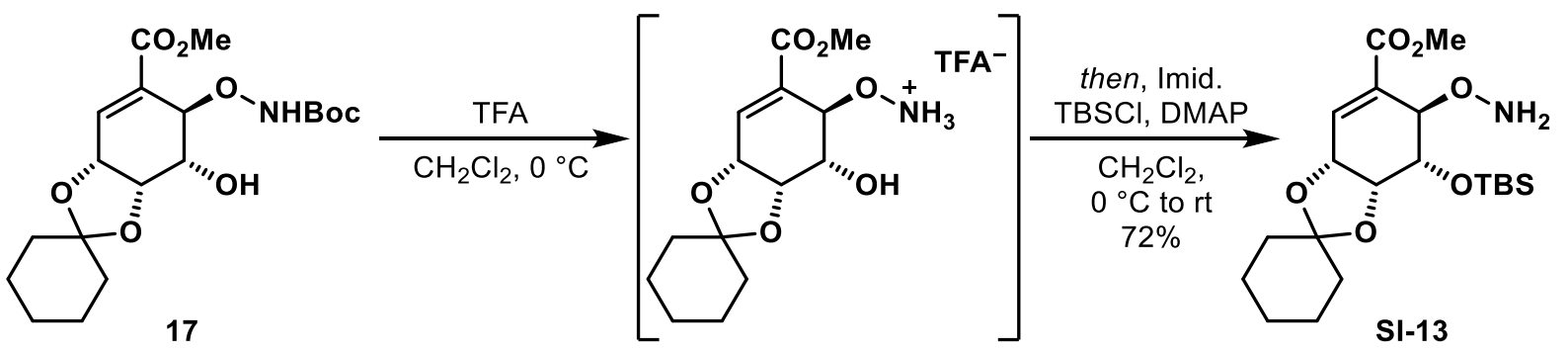

A $250 \mathrm{~mL}$ flame dried round-bottomed flask equipped with stir bar was charged with alcohol 17 ( $1.00 \mathrm{~g}, 2.50 \mathrm{mmol}, 1.00$ equiv.) and dissolved in $\mathrm{CH}_{2} \mathrm{Cl}_{2}$ (40 mL). The mixture was cooled to 0 ${ }^{\circ} \mathrm{C}$ and trifluoroacetic acid $(10 \mathrm{~mL})$ was slowly added. After $15 \mathrm{mins}$, anhydrous toluene $(50 \mathrm{~mL})$ was slowly added, the stir bar was removed and the reaction flask was transferred to a rotary evaporator and striped of solvent (Note 1). After drying under high vacuum a white foam was obtained which was treated with DMAP $(0.031$ g. $0.25 \mathrm{mmol}, 0.1$ equiv. $)$ and imidazole $(0.68 \mathrm{~g}$, $10 \mathrm{mmol}, 4.00$ equiv.). The flask was cooled to $0{ }^{\circ} \mathrm{C}$ and $\mathrm{CH}_{2} \mathrm{Cl}_{2}(15 \mathrm{~mL})$ was added with stirring. A $10 \mathrm{~mL}$ solution of TBSCl (1.13 g, $7.5 \mathrm{mmol}, 3.00$ equiv.) in $\mathrm{CH}_{2} \mathrm{Cl}_{2}$ was added via cannula transfer and the white, heterogeneous mixture was allowed to stir for 12 hours at room temperature. The mixture was diluted with $\mathrm{Et}_{2} \mathrm{O}(75 \mathrm{~mL})$ and poured into a separatory funnel containing 100 $\mathrm{mL}$ half saturated aqueous sodium bicarbonate. The layers were separated and the aqueous layer was extracted with an additional $75 \mathrm{~mL} \mathrm{Et}_{2} \mathrm{O}$. The combined organics were washed with brine $(100 \mathrm{~mL})$, dried with sodium sulfate and concentrated in-vacuo to provide a colorless oil. Column chromatography (50\% $\mathrm{Et}_{2} \mathrm{O}$ in hexanes) (Note 2) provided the title compound $\mathbf{S I - 1 3}$ as a viscous colorless resin $(0.743 \mathrm{~g}, 1.80 \mathrm{mmol}, 72 \%)$.

Note 1 - The rotovap bath was kept at $20{ }^{\circ} \mathrm{C}$. The reaction mixture was co-evaporated several times with toluene and once with ether to help remove residual TFA.

Note 2 - The product is somewhat unstable to silica gel and appears as two spots by TLC (major $R_{f}=0.3$, minor $R_{f}=0.6 ; 20 \%$ EtOAc in hexanes). Decomposition does not take place during column chromatography, and the product elutes between column volumes 3 and $4\left(R_{f}=0.3\right)$ using $50 \%$ ether in hexanes.

Physical state: clear, colorless resin.

TLC: $\mathrm{R}_{f}=0.3\left(20 \%\right.$ EtOAc in hexanes; visualization $\left.\mathrm{UV}, \mathrm{KMnO}_{4}\right)$ 
$[\alpha]_{D}^{20.0}=-53.9\left(c=1.0, \mathrm{CHCl}_{3}\right)$

${ }^{1}$ H NMR (500 MHz, CDCl $): \delta 6.77$ (d, $\left.J=2.3 \mathrm{~Hz}, 1 \mathrm{H}\right), 5.49$ (brs, 2H), $4.66-4.60$ (m, 1H), 4.58 $(\mathrm{d}, J=6.1 \mathrm{~Hz}, 1 \mathrm{H}), 4.38(\mathrm{dd}, J=5.8,3.1 \mathrm{~Hz}, 1 \mathrm{H}), 4.22(\mathrm{ddd}, J=6.1,3.1,0.9 \mathrm{~Hz}, 1 \mathrm{H}), 3.78$ (s, $3 \mathrm{H}), 1.62-1.52(\mathrm{~m}, 8 \mathrm{H}), 1.41-1.31(\mathrm{~m}, 2 \mathrm{H}), 0.89(\mathrm{~s}, 9 \mathrm{H}), 0.14(\mathrm{~s}, 3 \mathrm{H}), 0.10(\mathrm{~s}, 3 \mathrm{H})$.

${ }^{13}$ C NMR (125 MHz, CDCl3): $\delta$ 166.9, 138.0, 130.7, 110.7, 80.5, 75.3, 71.7, 68.5, 52.2, 36.8, $35.1,25.9,25.2,24.1,23.8,18.3,-4.3,-4.7$.

HRMS (m/z): calculated for $\mathrm{C}_{20} \mathrm{H}_{36} \mathrm{NO}_{6} \mathrm{Si}[\mathrm{M}+\mathrm{H}]^{+}:$414.2306, found 414.2311.

\section{Compound 5}
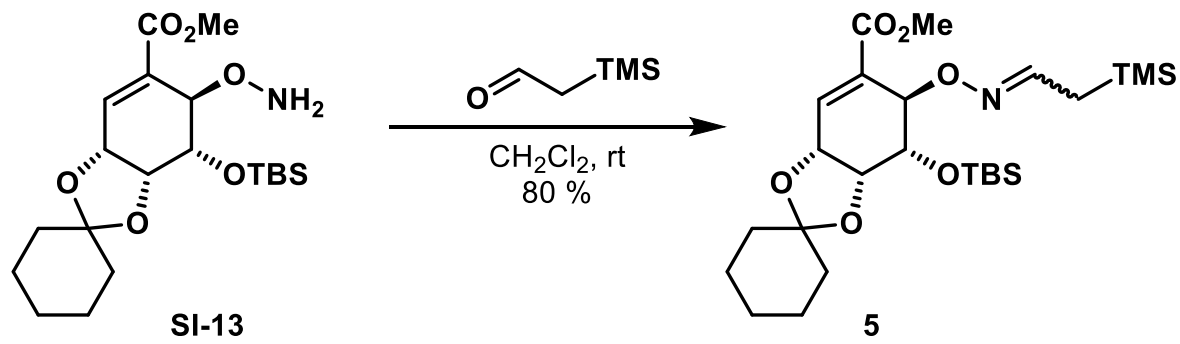

A dry $50 \mathrm{~mL}$ round-bottomed flask was charged with TMS-acetaldehyde $(13.7 \mathrm{~mL}, ~ 2.05 \mathrm{mmol}$, 1.20 equiv.) as a $0.15 \mathrm{M}$ solution in $\mathrm{CH}_{2} \mathrm{Cl}_{2}$ (Note 1). Amine SI-13 (0.706 g, $1.71 \mathrm{mmol}, 1.00$ equiv.) was dissolved in $\mathrm{CH}_{2} \mathrm{Cl}_{2}(7 \mathrm{~mL})$ and added dropwise to the aldehyde solution with stirring; the donor flask was rinsed with an additional $3 \mathrm{~mL}$ methylene chloride. After $30 \mathrm{mins}$, the mixture was poured into a separatory funnel containing $150 \mathrm{~mL} \mathrm{Et}_{2} \mathrm{O}$ and $100 \mathrm{~mL} \mathrm{H}_{2} \mathrm{O}$. The layers were separated, and the organic phase was washed with brine $(100 \mathrm{~mL})$, dried with sodium sulfate and concentrated in-vacuo. Purification by flash chromatography (15\% $\mathrm{Et}_{2} \mathrm{O}$ in hexanes) afforded oxime ether 5 ( $0.699 \mathrm{~g}, 1.37 \mathrm{mmol}, 80 \%$ yield $)$ as a 5:1 mixture of oxime isomers.

Note 1 - TMS-acetaldehyde is unstable and decomposes on isolation. It was prepared and used insitu from 2-(trimethylsilyl)ethanol. Procedure: A dry round-bottomed flask was charged with stir bar, iodobenzene diacetate ( $0.886 \mathrm{~g}, 2.75 \mathrm{mmol}, 1.1$ equiv.), TEMPO (0.039 g, $0.25 \mathrm{mmol}, 0.10$ equiv.) and $\mathrm{CH}_{2} \mathrm{Cl}_{2}(16.7 \mathrm{~mL}, 0.15 \mathrm{M})$. With stirring, 2-(trimethylsilyl)ethanol $(0.355 \mathrm{~mL}, 2.50$ mmol, 1.00 equiv.) was added. After 3 hours at room temperature, the oxidation was complete, and the crude aldehyde was used directly in the reaction.

Physical state: colourless to pale orange oil.

TLC: $\mathrm{R}_{f}=0.3\left(15 \% \mathrm{Et}_{2} \mathrm{O}\right.$ in hexanes; visualization $\left.\mathrm{UV}, \mathrm{KMnO}_{4}\right)$

$[\boldsymbol{\alpha}]_{D}^{\mathbf{2 0 . 0}}=-99.5\left(c=1.0, \mathrm{CHCl}_{3}\right)$

${ }^{1} \mathbf{H}$ NMR (600 MHz, CDCl $): \delta 7.37(\mathrm{t}, J=7.2 \mathrm{~Hz}, 0.83 \mathrm{H}), 7.22(\mathrm{~d}, J=2.7 \mathrm{~Hz}, 0.17 \mathrm{H}), 7.10(\mathrm{~d}$, $J=2.8 \mathrm{~Hz}, 0.83 \mathrm{H}), 6.51(\mathrm{t}, J=7.4 \mathrm{~Hz}, 0.17 \mathrm{H}), 5.62(\mathrm{~d}, J=4.9 \mathrm{~Hz}, 0.17 \mathrm{H}), 5.55(\mathrm{~d}, J=5.6 \mathrm{~Hz}$, $0.83 \mathrm{H}), 4.70(\mathrm{dd}, J=4.9,3.5 \mathrm{~Hz}, 0.17 \mathrm{H}), 4.64(\mathrm{dd}, J=5.6,3.4 \mathrm{~Hz}, 0.83 \mathrm{H}), 4.56(\mathrm{dd}, J=6.7,3.5$ 
$\mathrm{Hz}, 0.17 \mathrm{H}), 4.53(\mathrm{dd}, J=6.9,2.7 \mathrm{~Hz}, 0.17 \mathrm{H}), 4.50(\mathrm{dd}, J=6.2,3.4 \mathrm{~Hz}, 0.83 \mathrm{H}), 4.45(\mathrm{dd}, J=5.9$, $2.4 \mathrm{~Hz}, 0.83 \mathrm{H}), 3.41(\mathrm{~s}, 0.5 \mathrm{H}), 3.40(\mathrm{~s}, 2.5 \mathrm{H}), 1.85-1.80(\mathrm{~m}, 0.34 \mathrm{H}), 1.79-1.73(\mathrm{~m}, 1.66 \mathrm{H}), 1.71$ $-1.51(\mathrm{~m}, 6 \mathrm{H}), 1.41(\mathrm{dd}, J=13.5,7.4 \mathrm{~Hz}, 1 \mathrm{H}), 1.35(\mathrm{dd}, J=13.5,7.0 \mathrm{~Hz}, 1 \mathrm{H}), 1.28-1.20(\mathrm{~m}$, $2 \mathrm{H}), 1.05(\mathrm{~s}, 7.5 \mathrm{H}), 1.03(\mathrm{~s}, 1.5 \mathrm{H}), 0.28(\mathrm{~s}, 2.5 \mathrm{H}), 0.26(\mathrm{~s}, 0.5 \mathrm{H}), 0.22(\mathrm{~s}, 2.5 \mathrm{H}), 0.21(\mathrm{~s}, 0.5 \mathrm{H})$, $0.07(\mathrm{~s}, 1.5 \mathrm{H}),-0.08(\mathrm{~s}, 7.5 \mathrm{H})$.

${ }^{13}$ C NMR (150 MHz, CDCl3): $\delta 166.4,166.4,149.6,149.2,139.3,138.7,131.1,130.6,110.6$, 78.0, 77.9, 75.3, 74.6, 71.9, 71.5, 68.4, 67.6, 51.6, 51.5, 37.1, 36.9, 35.2, 34.9, 26.2, 25.6, 25.6, $24.4,24.3,24.2,24.2,19.9,18.9,18.6,-1.2,-1.7,-4.0,-4.1,-4.5,-4.7$.

HRMS (m/z): calculated for $\mathrm{C}_{25} \mathrm{H}_{46} \mathrm{NO}_{6} \mathrm{Si}_{2}[\mathrm{M}+\mathrm{H}]+512.2864$, found 512.2861.

\section{Compound des-TMS-5}
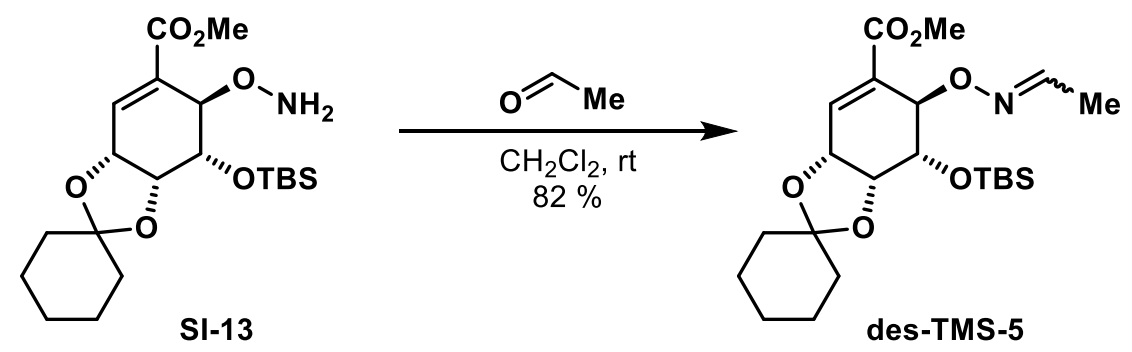

A dry culture tube containing amine SI-13 (50 mg, $0.12 \mathrm{mmol}, 1.00$ equiv.) was dissolved in $\mathrm{CH}_{2} \mathrm{Cl}_{2}\left(1.2 \mathrm{~mL}, 0.1 \mathrm{M}\right.$ ) and cooled to $0{ }^{\circ} \mathrm{C}$. Acetaldehyde (ca. $13 \mu \mathrm{L}, 0.24 \mathrm{mmol}, 2.00$ equiv.) was added and the mixture warmed to room temperature. After 15 mins, the reaction was concentrated in-vacuo and purified directly by preparative thin-layer chromatography $\left(30 \% \mathrm{Et}_{2} \mathrm{O}\right.$ in hexanes) to afford the title compound des-TMS-5 (43 mg, $0.10 \mathrm{mmol}, 82 \%$ yield) as 5:4 mixture of oxime isomers.

Physical state: colorless oil

TLC: $\mathrm{R}_{f}=0.3\left(20 \% \mathrm{Et}_{2} \mathrm{O}\right.$ in hexanes; visualization $\left.\mathrm{UV}, \mathrm{KMnO}_{4}\right)$

$[\alpha]_{D}^{20.0}=-89.3\left(c=1.0, \mathrm{CHCl}_{3}\right)$

${ }^{1}$ H NMR (500 MHz, C6D6): $\delta 7.13-7.06(\mathrm{~m}, 1.4 \mathrm{H}), 6.38(\mathrm{q}, J=5.5 \mathrm{~Hz}, 0.6 \mathrm{H}), 5.60(\mathrm{~d}, J=5.8$ $\mathrm{Hz}, 0.6 \mathrm{H}), 5.55(\mathrm{~d}, J=5.4 \mathrm{~Hz}, 0.4 \mathrm{H}), 4.59-4.51(\mathrm{~m}, 1 \mathrm{H}), 4.49-4.39(\mathrm{~m}, 2 \mathrm{H}), 3.37(\mathrm{~s}, 1.2 \mathrm{H})$, $3.37(\mathrm{~s}, 1.8 \mathrm{H}), 1.79-1.70(\mathrm{~m}, 2 \mathrm{H}), 1.64-1.52(\mathrm{~m}, 6 \mathrm{H}), 1.45(\mathrm{~d}, J=5.5 \mathrm{~Hz}, 1.8 \mathrm{H}), 1.37(\mathrm{~d}, J=$ $5.8 \mathrm{~Hz}, 1.2 \mathrm{H}), 1.27-1.20(\mathrm{~m}, 2 \mathrm{H}), 1.02(\mathrm{~s}, 5.4 \mathrm{H}), 1.01(\mathrm{~s}, 3.6 \mathrm{H}), 0.22(\mathrm{~s}, 1.2 \mathrm{H}), 0.21(\mathrm{~s}, 1.8 \mathrm{H})$, $0.18(\mathrm{~s}, 1.2 \mathrm{H}), 0.17(\mathrm{~s}, 1.8 \mathrm{H})$.

${ }^{13}$ C NMR (125 MHz, C6D6): $\delta$ 166.3, 166.3, 147.2, 147.1, 139.1, 138.8, 131.0, 130.6, 110.6, $110.6,78.4,78.1,75.4,75.0,71.9,71.7,68.6,68.2,51.6,51.5,37.1,37.0,35.3,35.1,26.1,25.6$, $25.5,24.3,24.2,18.5,15.0,11.8,-4.1,-4.2,-4.7$. 
HRMS (m/z): calculated for $\mathrm{C}_{22} \mathrm{H}_{38} \mathrm{NO}_{6} \mathrm{Si}[\mathrm{M}+\mathrm{H}]^{+}: 440.2463$, found 440.2479 .

\section{Compound 18}

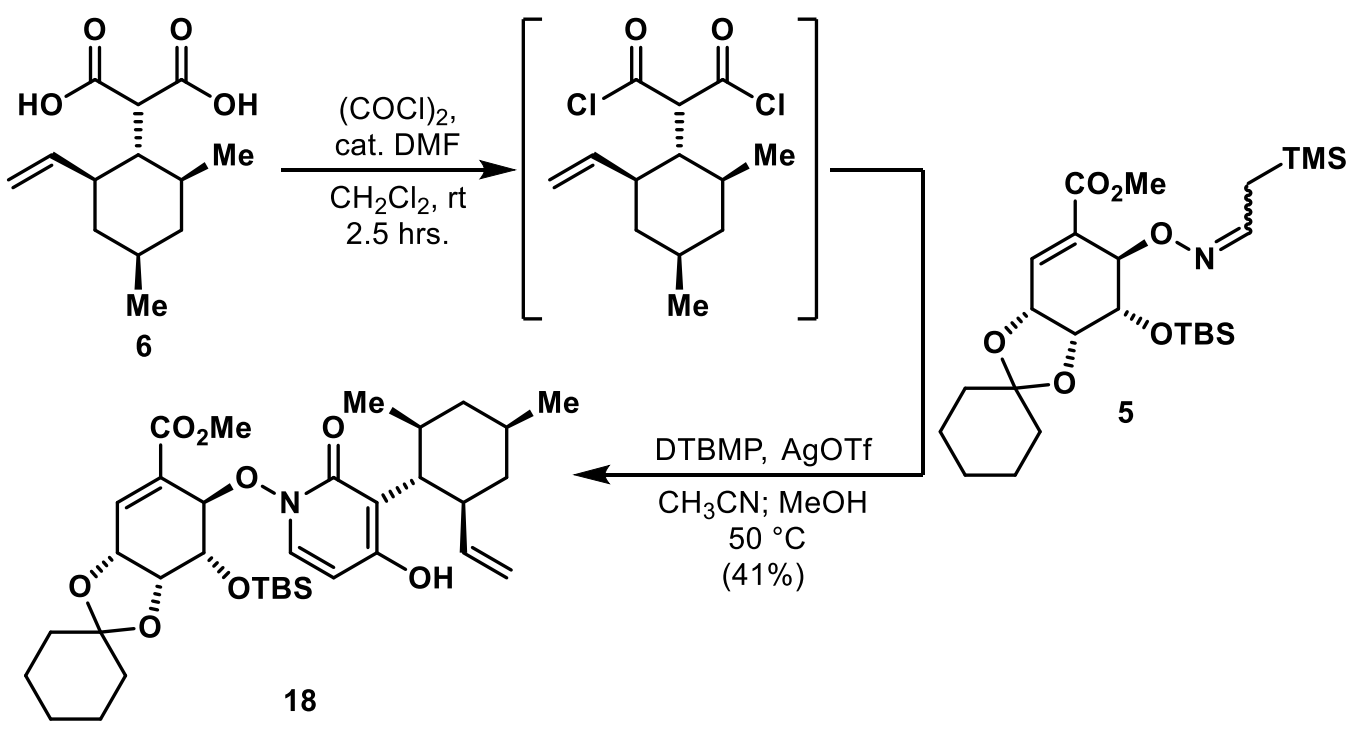

[Vessel A]-oxime ether + base solution: A medium sized flame dried culture tube equipped with stir bar was charged with oxime $\mathbf{5}(184 \mathrm{mg}, 0.36 \mathrm{mmol}, 1.20$ equiv.) and a small volume of benzene (ca. $0.1 \mathrm{~mL}$ ) was added. The tube was placed under high vacuum and allowed to dry overnight to a colorless resin. After starting the acid chloride formation (see vessel B), 2,6-di-tert-butyl-4methylpyridine (DTBMP) (135 mg, $0.66 \mathrm{mmol}, 2.20$ equiv.) was added to vessel A and it was placed under high vacuum for $c a .2$ hrs. It was subsequently placed under argon and $1 \mathrm{~mL}$ anhydrous $\mathrm{CH}_{3} \mathrm{CN}$ was added.

[Vessel B] - diacid chloride solution: A flame dried small culture tube was charged with diacid $\mathbf{6}$ (72 $\mathrm{mg}, 0.30 \mathrm{mmol}, 1.00$ equiv.) and the solid was dried under high vacuum for $30 \mathrm{mins}$. The vessel was backfilled with argon before $\mathrm{CH}_{2} \mathrm{Cl}_{2}(2 \mathrm{~mL})$ was added, followed by a $2 \mathrm{M}$ solution of oxalyl chloride in $\mathrm{CH}_{2} \mathrm{Cl}_{2}(0.38 \mathrm{~mL}, 0.75 \mathrm{mmol}, 2.50$ equiv.) (Note 1). With stirring, a solution of DMF in $\mathrm{CH}_{2} \mathrm{Cl}_{2}(0.15 \mathrm{~mL}, 0.024 \mathrm{mmol}, 0.08$ equiv.) (Note 2) was added. Gas evolution was immediately observed, and the heterogeneous mixture was stirred vigorously (1000 rpm) for 2.5 hours to produce a clear, pale yellow solution. Solvent was removed using a rotary evaporator (Note 3) and the apparatus was equipped with an argon balloon to enable back-filling under inert atmosphere. The vessel was briefly pulsed under high vacuum twice, backfilling with argon each time, the residue was then dissolved in anhydrous $\mathrm{CH}_{3} \mathrm{CN}(1.7 \mathrm{~mL})$.

[Vessel C]-silver triflate solution: A small oven dried culture tube was charged with silver triflate (146 mg, $0.57 \mathrm{mmol}, 1.90$ equiv.) (Note 4) and dissolved in $1.2 \mathrm{~mL}$ anhydrous $\mathrm{CH}_{3} \mathrm{CN}$.

Fragment union: To the oxime ether + base solution [vessel A] the diacid chloride solution [vessel B] was added via syringe and chased with a $0.3 \mathrm{~mL} \mathrm{CH}_{3} \mathrm{CN}$ rinse. The mixture was quickly 
transferred to an oil bath at $50{ }^{\circ} \mathrm{C}$ with vigorous stirring $(1200 \mathrm{rpm})$. The flask contents were allowed to warm in the bath for ca. 20 seconds before the silver triflate solution [vessel C] was added via syringe in a steady stream (Note 5). The mixture was allowed to stir at this temperature for 7 minutes before removing from oil bath and allowing to cool ( 2 minutes). With stirring, anhydrous methanol $(0.6 \mathrm{~mL})$ was added and the mixture was allowed to stir for 2 minutes. The vessel contents were diluted with EtOAc and transferred to a separatory funnel. The organic layer was washed with $0.2 \mathrm{M} \mathrm{HCl}(15 \mathrm{~mL})$ and the layers separated. The aqueous layer was backextracted with EtOAc and the combined organics were washed with brine $(10 \mathrm{~mL})$, dried with sodium sulfate and concentrated in-vacuo to obtain a solid residue. This residue was taken up in $\mathrm{CH}_{2} \mathrm{Cl}_{2}$, filtered and purified by preparative thin layer chromatography ( $5 \%$ acetone in $\mathrm{CH}_{2} \mathrm{Cl}_{2}$ ) to afford the condensation product $18(80 \mathrm{mg}, 0.124 \mathrm{mmol}, 41 \%$ yield) as a 1:1 mixture of atropisomers.

Note 1 - Oxalyl chloride was purchased as a $2 \mathrm{M}$ solution in $\mathrm{CH}_{2} \mathrm{Cl}_{2}$ from Sigma Aldrich. We found this reagent to provide cleaner acid chloride formation compared with either neat $(\mathrm{COCl})_{2}$ or from a self-prepared stock solution. The bottle was stored at $7^{\circ} \mathrm{C}$ and allowed to warm to room temperature prior to use.

Note 2 - DMF stock solution was prepared by adding $60 \mu \mathrm{L} \mathrm{DMF}$ to $5 \mathrm{~mL} \mathrm{CH}_{2} \mathrm{Cl}_{2}$.

Note 3 - The inside of the rotovap was rinsed with acetone, then hexanes prior to removing the reaction solvent. The bath was kept at $25{ }^{\circ} \mathrm{C}$ and a neodymium magnet was used to hold the stir bar above the solvent to prevent bumping.

Note 4 - Silver triflate was stored and weighed in a glovebox.

Note 5 - It is important to add the silver triflate solution reasonably quickly - the addition took approximately 10 seconds. It was found that rapid addition does not affect yield significantly, however slow dropwise addition decreases yield.

Physical state: White amorphous solid.

TLC: $\mathrm{R}_{f}=0.5\left(5 \%\right.$ acetone in $\mathrm{CH}_{2} \mathrm{Cl}_{2}$, visualization $\left.\mathrm{UV}, \mathrm{KMnO}_{4}\right)$

$[\boldsymbol{\alpha}]_{D}^{20.0}=-157.2\left(c=1.0, \mathrm{CHCl}_{3}\right)$

${ }^{1}$ H NMR (600 MHz, CDCl $): \delta 10.27(\mathrm{~s}, 0.5 \mathrm{H}), 9.81(\mathrm{~s}, 0.5 \mathrm{H}), 7.48(\mathrm{~d}, J=7.9 \mathrm{~Hz}, 0.5 \mathrm{H}), 7.46$ $(\mathrm{d}, J=7.9 \mathrm{~Hz}, 0.5 \mathrm{H}), 7.43-7.39(\mathrm{~m}, 1 \mathrm{H}), 6.32(\mathrm{~d}, J=7.8 \mathrm{~Hz}, 0.5 \mathrm{H}), 6.29(\mathrm{~d}, J=7.8 \mathrm{~Hz}, 0.5 \mathrm{H})$, $5.64(\mathrm{dt}, J=17.9,9.7 \mathrm{~Hz}, 0.5 \mathrm{H}), 5.51(\mathrm{dt}, J=18.1,9.3 \mathrm{~Hz}, 0.5 \mathrm{H}), 5.04(\mathrm{~d}, J=3.4 \mathrm{~Hz}, 0.5 \mathrm{H}), 5.01$ $(\mathrm{d}, J=3.4 \mathrm{~Hz}, 0.5 \mathrm{H}), 4.90(\mathrm{dd}, J=7.3,4.0 \mathrm{~Hz}, 0.5 \mathrm{H}), 4.87-4.82(\mathrm{~m}, 1 \mathrm{H}), 4.76(\mathrm{dd}, J=7.4,2.8$ $\mathrm{Hz}, 0.5 \mathrm{H}), 4.74-4.70(\mathrm{~m}, 0.75 \mathrm{H}), 4.71-4.66(\mathrm{~m}, 0.75 \mathrm{H}), 4.58(\mathrm{~d}, J=11.7 \mathrm{~Hz}, 0.5 \mathrm{H}), 4.50-4.44$ $(\mathrm{m}, 1 \mathrm{H}), 3.75(\mathrm{~s}, 1.5 \mathrm{H}), 3.74(\mathrm{~s}, 1.5 \mathrm{H}), 2.98-2.85(\mathrm{~m}, 1 \mathrm{H}), 2.66(\mathrm{t}, J=11.0 \mathrm{~Hz}, 0.5 \mathrm{H}), 2.48(\mathrm{~d}, \mathrm{~J}$ $=6.0 \mathrm{~Hz}, 1 \mathrm{H}), 2.30-2.20(\mathrm{~m}, 0.5 \mathrm{H}), 1.80-1.48(\mathrm{~m}, 11 \mathrm{H}), 1.37(\mathrm{~s}, 2 \mathrm{H}), 0.95-0.92(\mathrm{~m}, 1 \mathrm{H}), 0.91$ $(\mathrm{d}, J=6.1 \mathrm{~Hz}, 1.5 \mathrm{H}), 0.89(\mathrm{~d}, J=6.3 \mathrm{~Hz}, 1.5 \mathrm{H}), 0.76-0.75(\mathrm{~m}, 11.5 \mathrm{H}), 0.71(\mathrm{~d}, J=6.4 \mathrm{~Hz}, 1.5 \mathrm{H})$, $0.03(\mathrm{~s}, 1.5 \mathrm{H}), 0.01(\mathrm{~s}, 1.5 \mathrm{H}),-0.04(\mathrm{~s}, 3 \mathrm{H})$.

${ }^{13}$ C NMR (150 MHz, CDCl3): $\delta$ 166.0, 166.0, 163.7, 163.1, 162.3, 159.8, 143.5, 143.3, 142.9, $142.8,132.8,132.6,126.6,126.5,115.4,115.2$, 113.0, 110.8, 110.8, 101.8, 100.6, 82.6, 82.4, 72.3, 
70.0, 70.0, 64.1, 64.0, 52.4, 52.4, 46.9, 46.6, 44.7, 44.6, 43.4, 43.3, 42.4, 42.3, 35.7, 34.1, 34.0, $33.0,31.9,31.9,31.8,25.9,25.3,24.0,23.8,22.9,22.8,20.8,20.7,18.1,18.1,-4.3,-4.3,-5.6$, 5.6 .

HRMS (m/z): calculated for $\mathrm{C}_{35} \mathrm{H}_{54} \mathrm{NO}_{8} \mathrm{Si}[\mathrm{M}+\mathrm{H}]^{+}:$644.3613, found 644.3616.
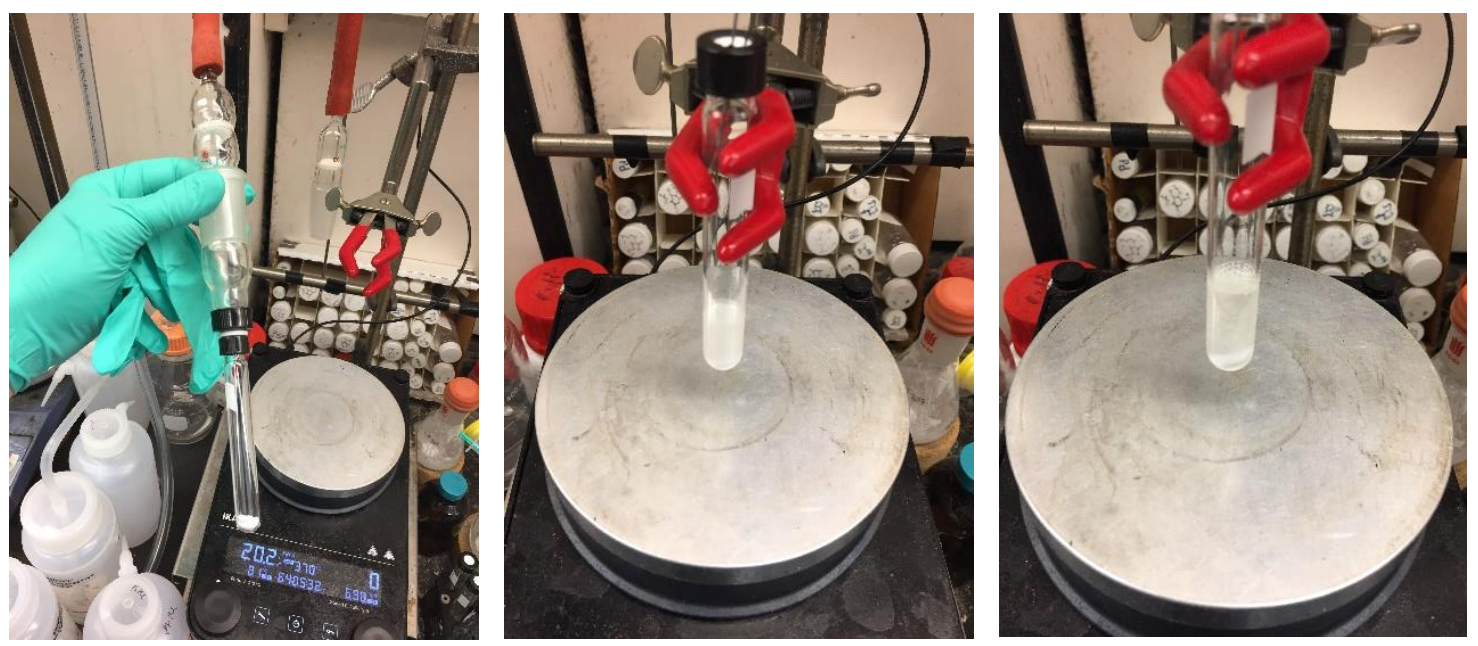

[Vessel B]: (left) drying diacid under high vacuum, (middle) diacid dissolved in $\mathrm{CH}_{2} \mathrm{Cl}_{2}$, (right) after addition of $(\mathrm{COCl})_{2}$ and $\mathrm{DMF}$.
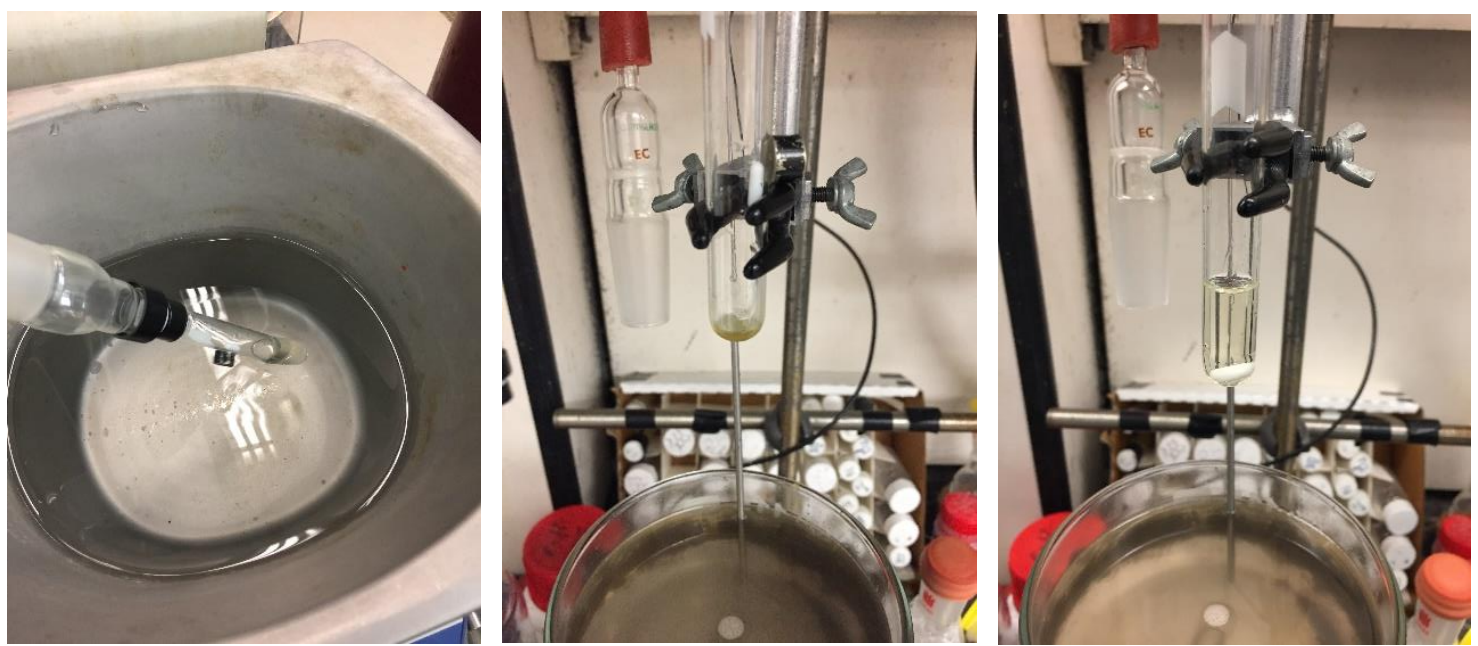

[Vessel B]: (left) solvent removal using rotovap, (middle) crude diacid chloride, (right) diacid chloride dissolved in $\mathrm{CH}_{3} \mathrm{CN}$. 

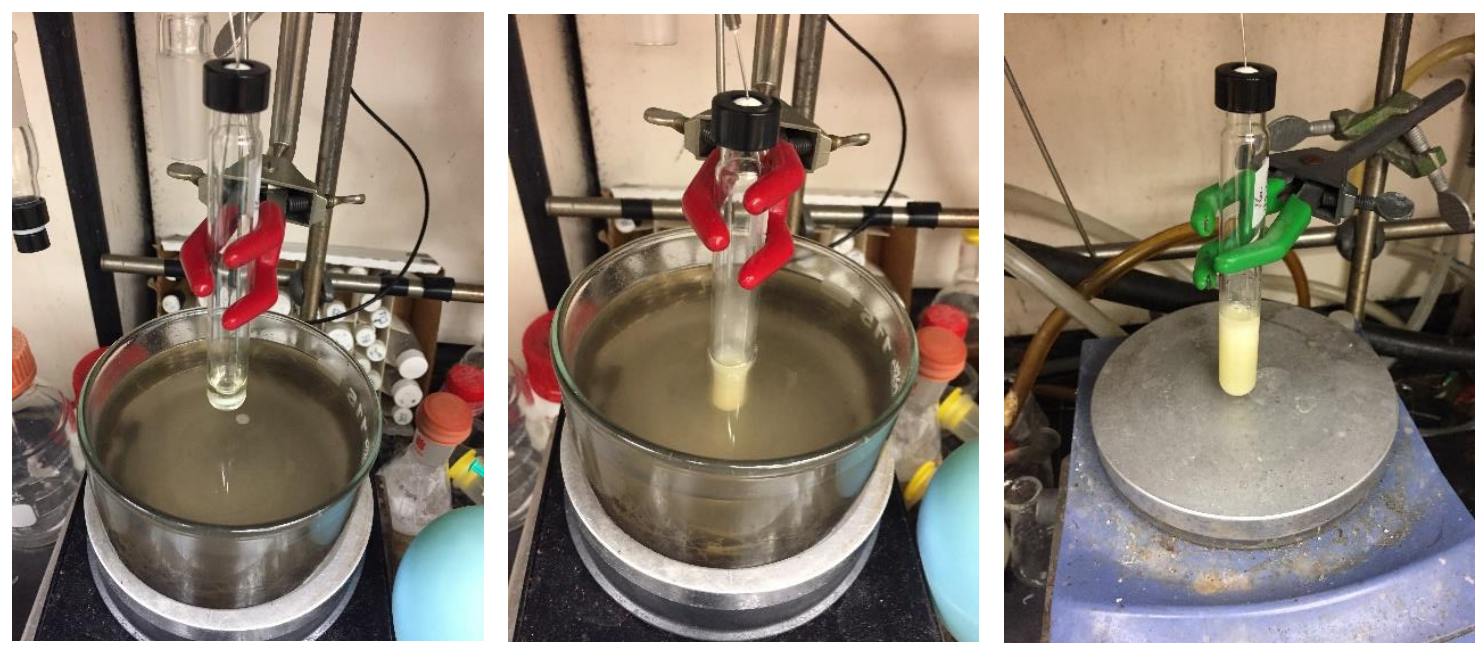

[Vessel A]: (left) oxime + base dissolved in $\mathrm{CH}_{3} \mathrm{CN}$, (middle) reaction mixture after adding diacid chloride and AgOTf, (right) reaction after methanol quench.

\section{Compound 1 - Maximiscin}
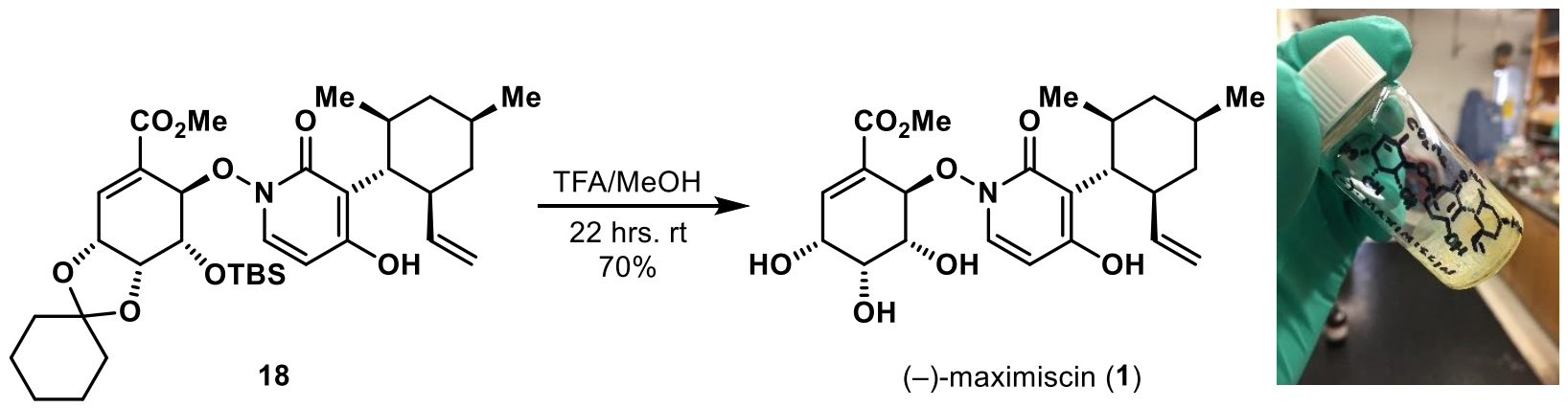

To a 5 dram vial containing 18 (115 $\mathrm{mg}, 0.179 \mathrm{mmol}, 1.00$ equiv.) was added anhydrous methanol (3 mL) (Note 1). The mixture was cooled to $0{ }^{\circ} \mathrm{C}$ and trifluoroacetic acid ( $3 \mathrm{~mL}$ ) (Note 2) was added dropwise. The vessel was sealed with a screw cap and allowed to stir at room temperature until complete conversion was achieved ( $c a .22 \mathrm{hrs,} \mathrm{monitored} \mathrm{by} \mathrm{LCMS).} \mathrm{Solvent} \mathrm{was} \mathrm{removed}$ in-vacuo and the residue was dried under high vacuum to yield a viscus oil. This was taken up in $5 \% \mathrm{MeOH}$ in $\mathrm{CH}_{2} \mathrm{Cl}_{2}$ and purified by preparative thin layer chromatography (10\% methanol in $\mathrm{CH}_{2} \mathrm{Cl}_{2}$ ) to afford the natural product (-)-maximiscin $\mathbf{1}^{14}$ (56 mg, $0.125 \mathrm{mmol}, 70 \%$ yield) (Note 3). [Collectively, over $100 \mathrm{mg}$ of 1 was prepared over several batches]

Note 1 - Maximiscin is extremely sensitive to $\mathrm{Fe}^{3+}$ salts and can decompose in the presence of iron leached from metal needles. HPLC grade methanol was used, transferred using a glass pipette.

Note 2 - See above. HPLC grade TFA was used, transferred using glass pipette.

Note 3 - The remaining mass balance was the cyclic ketal protected compound SI-13 shown below. On larger scale, due to the slower solvent removal process, some residual TFA was able to catalyze the re-protection of $\mathbf{1}$. This material could be isolated ( $25 \mathrm{mg}, 0.047 \mathrm{mmol}, 26 \%$ yield) and re-subjected to the deprotection conditions to obtain additional $\mathbf{1}$. 
<smiles>[M]C1CC(C)C(c2c(O)ccn(OC3C(C(C)=O)=C[C@@H](O)[C@H](O)[C@H]3O)c2=O)C(C=C)C1</smiles>

Physical state: pale yellow solid

m.p.: $106-109^{\circ} \mathrm{C}($ dec. $)$

TLC: $\mathrm{R}_{f}=0.5\left(10 \% \mathrm{MeOH}\right.$ in $\left.\mathrm{CH}_{2} \mathrm{Cl}_{2}\right)$

$[\alpha]_{D}^{20.0}=-146.0(c 0.50, \mathrm{MeOH})$

${ }^{1}$ H NMR (600 MHz, DMSO-d6): see table 18

${ }^{13}$ C NMR (150 MHz, DMSO-d6): see table 19

HRMS (m/z): calculated for $\mathrm{C}_{23} \mathrm{H}_{32} \mathrm{NO}_{8}{ }^{+}[\mathrm{M}+\mathrm{H}]^{+}: 450.2122$, found 450.2138 .

LCMS Co-inject Parameters: The sample was analyzed on a Waters I-Class with a Waters Cortecs C18 column $(1.6 \mu \mathrm{m}, 2.1 \times 55 \mathrm{~mm})$ under isocratic conditions $(0.75 \mathrm{~mL} / \mathrm{min}, 25 \%$ acetonitrile / [0.1\% aqueous formic acid]) at $10{ }^{\circ} \mathrm{C}$. The compounds were detected by UV light $(290 \mathrm{~nm})$.

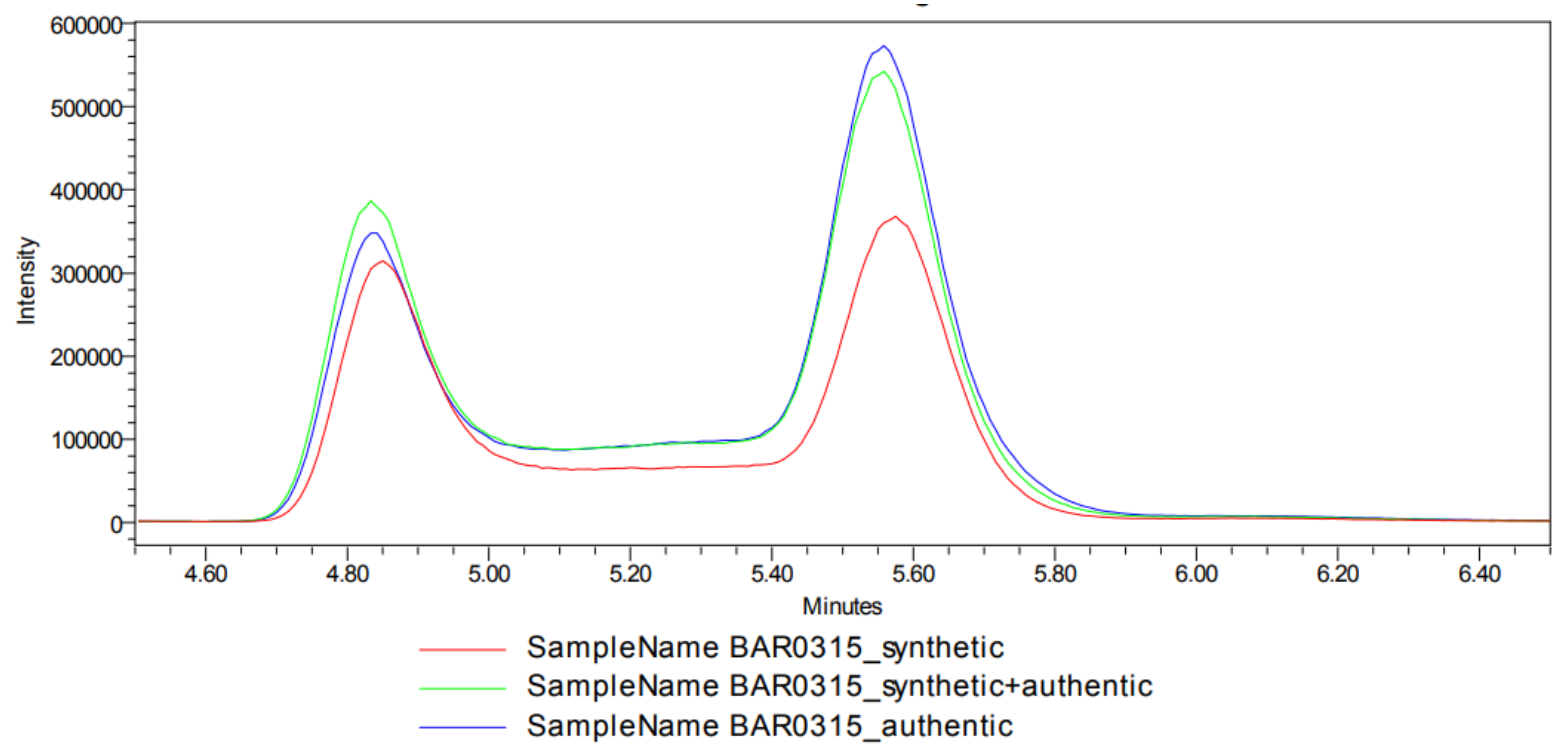


${ }^{1}$ H NMR Overlay (natural top, synthetic bottom):
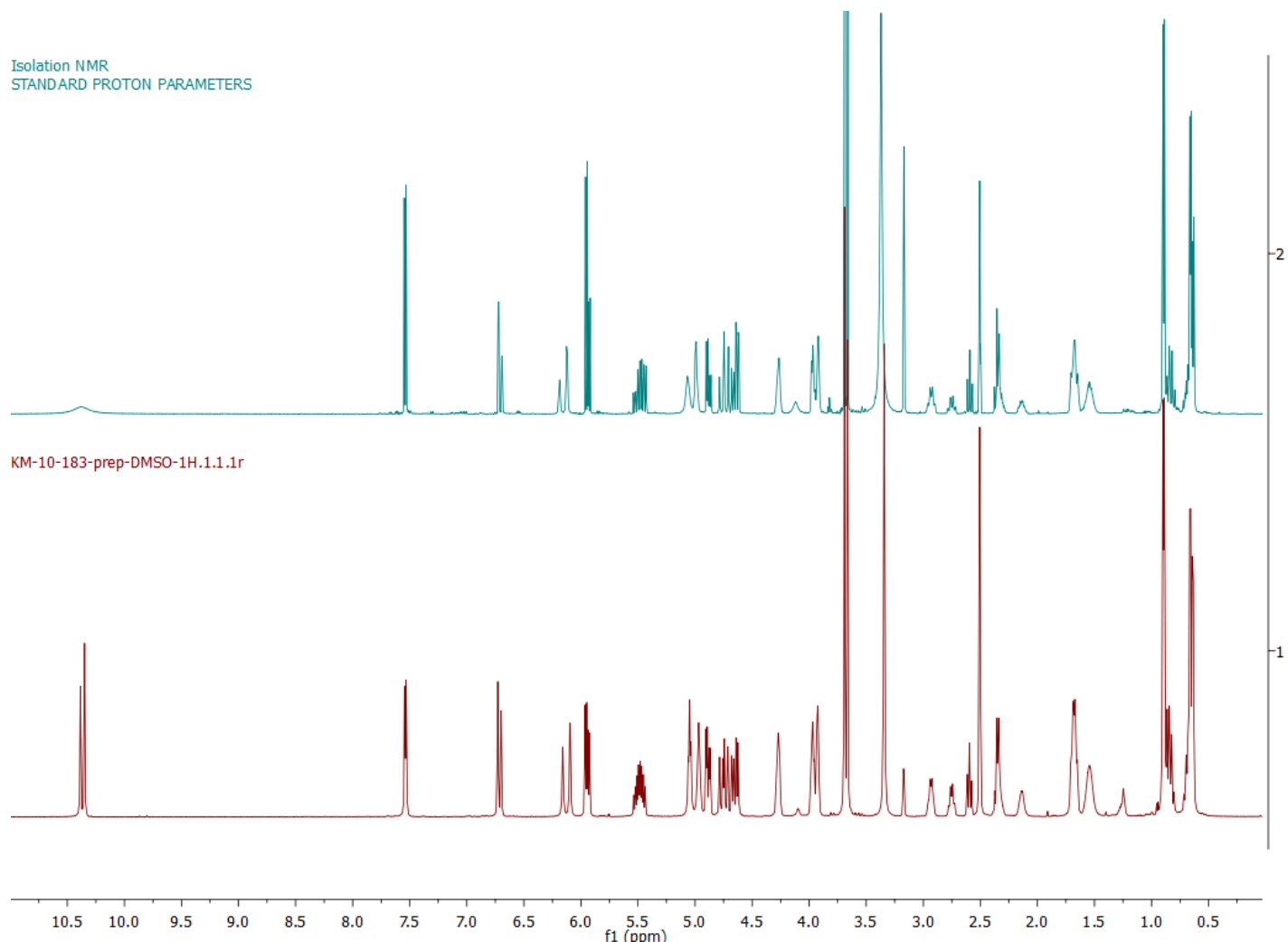

${ }^{13}$ C NMR Overlay (natural top, synthetic bottom):

STANDARD PROTON PARAMETERS

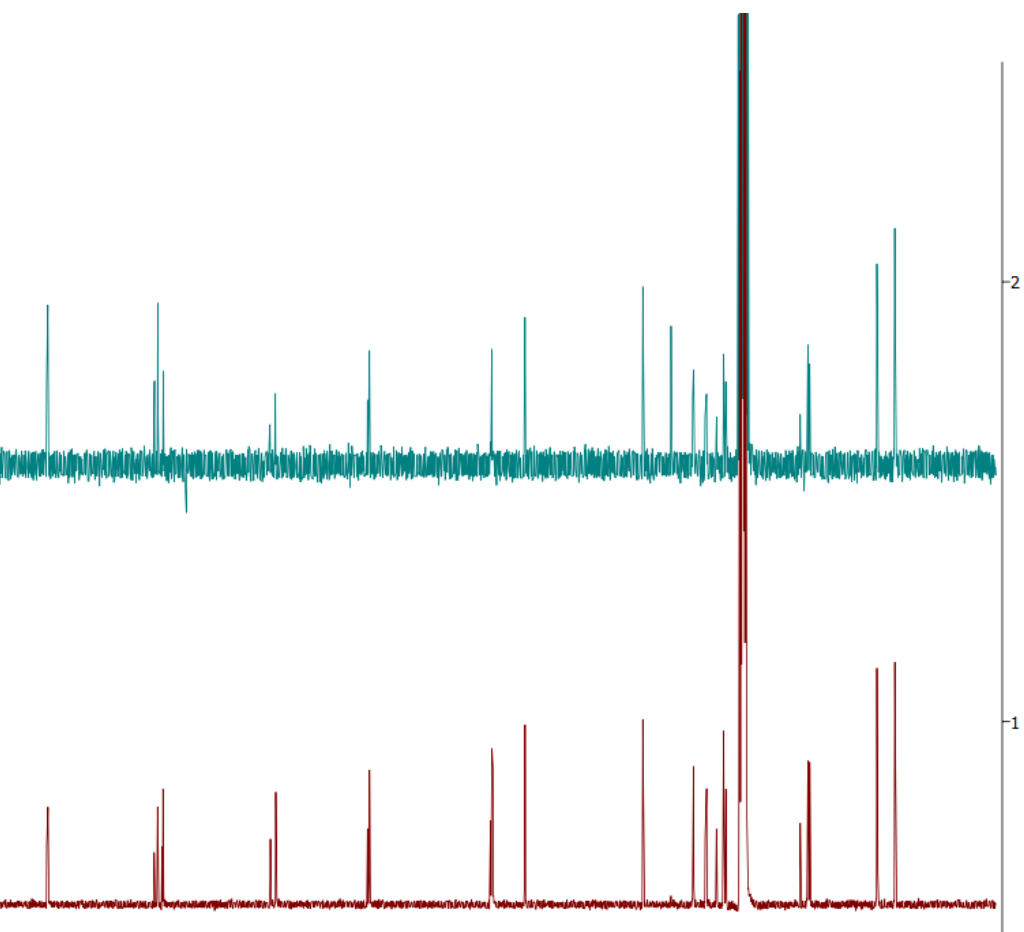

180

$170 \quad 160$

${ }_{\mathrm{f} 1(\mathrm{ppm})}^{90}$

80

SI-87 
Table 18. ${ }^{1} \mathrm{H}$ NMR data for synthetic (-)-maximiscin compared to reported spectra. ${ }^{14}$

\begin{tabular}{|c|c|c|c|c|}
\hline \multirow[t]{2}{*}{ No. } & \multicolumn{2}{|c|}{$P-1 \delta_{H}(p p m)$} & \multicolumn{2}{|c|}{$M-1 \delta_{H}(p p m)$} \\
\hline & Lit & Found $(J$ in $\mathrm{Hz})$ & Lit & Found $(J$ in $\mathrm{Hz})$ \\
\hline $4(\mathrm{OH})$ & $10.37, \mathrm{~s}$ & 10.38, brs & 10.37, brs & $10.34 \mathrm{~s}$ \\
\hline 5 & $5.95, \mathrm{~d}(7.8)$ & $5.95, \mathrm{~d}(7.9)$ & $5.91, \mathrm{~d}(7.8)$ & $5.93, \mathrm{~d}(7.9)$ \\
\hline 6 & $7.53, \mathrm{~d}(7.8)$ & $7.53, \mathrm{~d}(7.8)$ & $7.52, \mathrm{~d}(7.8)$ & $7.52, \mathrm{~d}(7.9)$ \\
\hline 7 & $2.36, \mathrm{t}(10.5)$ & $2.35, \mathrm{t}(10.7)$ & $2.59, \mathrm{t}(10.5)$ & $2.59, \mathrm{t}(11.1)$ \\
\hline 8 & $2.93, \mathrm{~m}$ & $2.93, \mathrm{~m}$ & $2.74, \mathrm{~m}$ & $2.75, \mathrm{~m}$ \\
\hline $9 \mathrm{a}$ & $1.68, \mathrm{~m}$ & $1.68, \mathrm{~m}$ & $1.70, \mathrm{~m}$ & $1.70, \mathrm{~m}$ \\
\hline $9 b$ & $0.86, \mathrm{~m}$ & $0.86, \mathrm{~m}$ & $0.81, \mathrm{~m}$ & $0.81, \mathrm{~m}$ \\
\hline 10 & $1.53, \mathrm{~m}$ & $1.54, \mathrm{~m}$ & $1.53, \mathrm{~m}$ & $1.54, \mathrm{~m}$ \\
\hline $11 \mathrm{a}$ & $1.67, \mathrm{~m}$ & $1.67, \mathrm{~m}$ & $1.67, \mathrm{~m}$ & $1.67, \mathrm{~m}$ \\
\hline $11 b$ & $0.69, \mathrm{~m}$ & $0.69, \mathrm{~m}$ & $0.69, \mathrm{~m}$ & $0.69, \mathrm{~m}$ \\
\hline 12 & $2.32, \mathrm{~m}$ & $2.33, \mathrm{~m}$ & $2.15, \mathrm{~m}$ & $2.13, \mathrm{~m}$ \\
\hline 13 & $5.40, \mathrm{~m}$ & $5.48, \mathrm{~m}$ & $5.53, \mathrm{~m}$ & $5.48, \mathrm{~m}$ \\
\hline $14 \mathrm{a}$ & $\begin{array}{c}4.73, \mathrm{dd}(16.5 \\
1.8)\end{array}$ & $4.72, \mathrm{~d}(17.3)$ & $\begin{array}{c}4.75, \text { dd }(16.5 \\
1.8)\end{array}$ & $4.76, \mathrm{~d}(17.4)$ \\
\hline $14 b$ & $\begin{array}{l}\text { 4.62, dd }(10.3, \\
1.8)\end{array}$ & $\begin{array}{c}4.63 \text {, dd }(10.2, \\
1.4)\end{array}$ & $\begin{array}{l}\text { 4.66, dd }(10.3 \text {, } \\
1.8)\end{array}$ & 4.66, dd $(10.3$ \\
\hline 15 & $0.89, \mathrm{~d}(6.2)$ & $0.89, \mathrm{~d}(6.1)$ & $0.89, \mathrm{~d}(6.2)$ & $0.89, \mathrm{~d}(6.1)$ \\
\hline 16 & $0.65, \mathrm{~d}(6.2)$ & $0.66, \mathrm{~d}(5.6)$ & $0.62, \mathrm{~d}(6.2)$ & $0.63, \mathrm{~d}(6.5)$ \\
\hline $2 ’$ & $\begin{array}{c}6.72, \text { ddd }(2.5, \\
1.0,1.0)\end{array}$ & $6.72, \mathrm{~m}$ & $\begin{array}{c}6.69 \text {, ddd }(2.5 \\
1.0,1.0)\end{array}$ & $6.69, \mathrm{~m}$ \\
\hline 3 & 4.26, brs & 4.27, brs & 4.26 , brs & 4.27, brs \\
\hline $3^{\prime}(\mathrm{OH})$ & n.r. & $5.04, \mathrm{~m}$ & n.r. & $5.04, \mathrm{~m}$ \\
\hline $4^{\prime}$ & 3.92, brs & 3.92, brs & 3.92, brs & 3.92, brs \\
\hline $4^{\prime}(\mathrm{OH})$ & 4.96, brs & 4.96, brs & 4.96, brs & 4.96, brs \\
\hline 5 & $\begin{array}{c}3.96 \mathrm{dd}(1.5 \\
6.2)\end{array}$ & $3.96, \mathrm{~m}$ & $\begin{array}{c}3.96 \mathrm{dd}(1.5 \\
6.2)\end{array}$ & $3.96, \mathrm{~m}$ \\
\hline $5^{\prime}(\mathrm{OH})$ & 6.10, brs & $6.10, \mathrm{~d}(2.3)$ & 6.16, brs & 6.15, brs \\
\hline 6 & $\begin{array}{c}4.89, \text { ddd }(6.2 \\
1.0,0.9)\end{array}$ & $4.89, \mathrm{~d}(6.1)$ & $\begin{array}{c}4.87 \text {, ddd }(6.2 \text {, } \\
1.0,0.9)\end{array}$ & $4.87, \mathrm{~d}(6.1)$ \\
\hline 8 & $3.68, \mathrm{~s}$ & $3.69, \mathrm{~s}$ & $3.66, \mathrm{~s}$ & $3.66, \mathrm{~s}$ \\
\hline
\end{tabular}


Table 19. ${ }^{13} \mathrm{C}$ NMR data for synthetic (-)-maximiscin compared to reported spectra. ${ }^{14}$

\begin{tabular}{|c|c|c|c|c|}
\hline \multirow[t]{2}{*}{ No. } & \multicolumn{2}{|c|}{$P-1 \delta_{C}(p p m)$} & \multicolumn{2}{|c|}{$M-1 \delta_{C}(p p m)$} \\
\hline & Lit & Found $(\Delta)$ & Lit & Found $(\Delta)$ \\
\hline 2 & 159.3 & 159.3 & 161.5 & 161.5 \\
\hline 3 & 113.1 & $113.2(0.1)$ & 113.6 & 113.6 \\
\hline 4 & 162.5 & $162.4(0.1)$ & 161.5 & $161.4(0.1)$ \\
\hline 5 & 98.4 & $98.3(0.1)$ & 99.0 & 99.0 \\
\hline 6 & 134.2 & 134.2 & 134.0 & 134.0 \\
\hline 7 & 45.8 & 45.8 & 45.9 & 45.9 \\
\hline 8 & 42.0 & 42.0 & 42.9 & 42.9 \\
\hline $9 \mathrm{a}$ & 41.7 & 41.7 & 42.0 & 42.0 \\
\hline \multicolumn{5}{|l|}{$9 b$} \\
\hline 10 & 31.4 & 31.4 & 31.4 & 31.4 \\
\hline $11 \mathrm{a}$ & 44.1 & 44.1 & 44.3 & 44.3 \\
\hline \multicolumn{5}{|l|}{$11 b$} \\
\hline 12 & 31.2 & 31.2 & 32.4 & 32.4 \\
\hline 13 & 143.3 & 143.3 & 143.1 & 143.1 \\
\hline $14 \mathrm{a}$ & 112.5 & 112.5 & 112.6 & 112.6 \\
\hline \multicolumn{5}{|l|}{$14 b$} \\
\hline 15 & 22.7 & 22.7 & 22.6 & 22.6 \\
\hline 16 & 20.5 & 20.5 & 20.4 & 20.4 \\
\hline $1^{\prime}$ & 127.0 & 127.0 & 127.1 & 127.1 \\
\hline $2^{\prime}$ & 145.7 & 145.7 & 145.5 & 145.5 \\
\hline $3^{\prime}$ & 67.0 & 67.0 & 67.0 & 67.0 \\
\hline $4^{\prime}$ & 71.1 & 71.1 & 71.2 & 71.2 \\
\hline 5 & 71.0 & 71.0 & 71.3 & 71.3 \\
\hline 6 & 86.5 & 86.5 & 86.8 & 86.8 \\
\hline 7 & 166.0 & 166.0 & 166.0 & 166.0 \\
\hline 8 & 52.1 & $52.2(0.1)$ & 52.0 & $52.1(0.1)$ \\
\hline
\end{tabular}




\section{Miscellaneous Data \\ Compound SI-1}

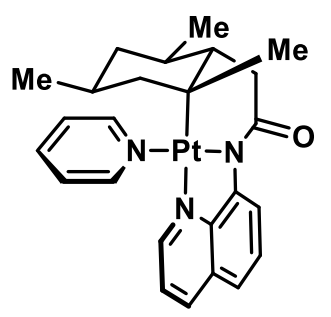

SI-1

Physical state: yellow solid

m.p.: n.d.

TLC: $\mathrm{R}_{f}=0.2(90 \%$ EtOAc in hexanes; visualization UV)

${ }^{1} \mathbf{H}$ NMR $\left(600 \mathrm{MHz}, \mathbf{C}_{6} \mathbf{D}_{6}\right): \delta 10.17(\mathrm{~d}, J=8.2 \mathrm{~Hz}, 1 \mathrm{H}), 8.66(\mathrm{~d}, J=5.6 \mathrm{~Hz}, 1 \mathrm{H}), 8.21(\mathrm{~d}, J=5.6$ $\mathrm{Hz}, 1 \mathrm{H}), 7.48(\mathrm{dd}, J=8.2,1.4 \mathrm{~Hz}, 1 \mathrm{H}), 7.38(\mathrm{t}, J=8.1 \mathrm{~Hz}, 1 \mathrm{H}), 6.83(\mathrm{dd}, J=7.9,0.8 \mathrm{~Hz}, 1 \mathrm{H})$, $6.67(\mathrm{tt}, J=7.7,1.5 \mathrm{~Hz}, 1 \mathrm{H}), 6.62(\mathrm{dd}, J=4.8,1.5 \mathrm{~Hz}, 1 \mathrm{H}), 6.36-6.33(\mathrm{~m}, 1 \mathrm{H}), 6.32(\mathrm{dd}, J=8.2$, $4.8 \mathrm{~Hz}, 1 \mathrm{H}), 6.22-6.16(\mathrm{~m}, 1 \mathrm{H}), 3.52(\mathrm{dd}, J=15.8,2.3 \mathrm{~Hz}, 1 \mathrm{H}), 3.32(\mathrm{dd}, J=15.7,5.0 \mathrm{~Hz}, 1 \mathrm{H})$, $2.72-2.62(\mathrm{~m}, 1 \mathrm{H}), 2.42-2.33(\mathrm{~m}, 1 \mathrm{H}), 1.87(\mathrm{dq}, J=11.1,2.9,2.5 \mathrm{~Hz}, 1 \mathrm{H}), 1.46(\mathrm{~d}, J=6.5 \mathrm{~Hz}$, $3 \mathrm{H}), 1.28(\mathrm{~s}, 3 \mathrm{H}), 0.92(\mathrm{q}, J=12.0 \mathrm{~Hz}, 1 \mathrm{H}), 0.85(\mathrm{~d}, J=6.7 \mathrm{~Hz}, 3 \mathrm{H}), 0.78(\mathrm{dt}, J=13.4,2.5 \mathrm{~Hz}$, $1 \mathrm{H}), 0.56(\mathrm{ddd}, J=10.5,4.9,2.2 \mathrm{~Hz}, 1 \mathrm{H}), 0.22-0.14(\mathrm{~m}, 1 \mathrm{H})$.

\section{X-ray Structure SI-1:}

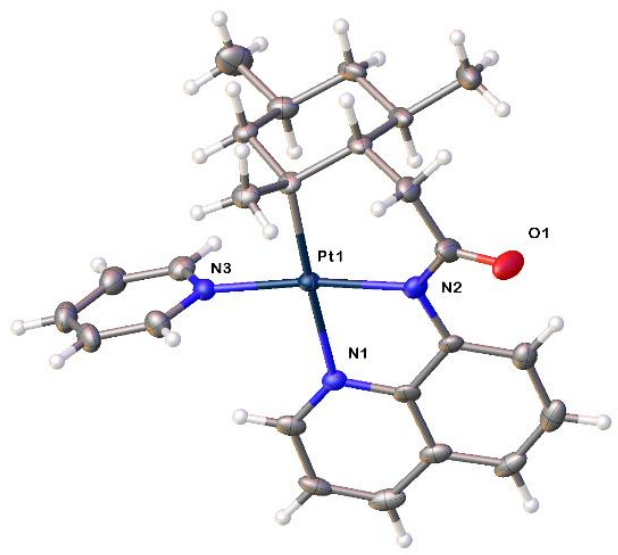

Experimental Summary

The single crystal X-ray diffraction studies were carried out on a Bruker APEX II Ultra CCD diffractometer equipped with $M o K_{\alpha}$ radiation $(\lambda 0.71073 \AA$ ).

Crystals of the subject compound were used as received (grown from benzene/hexanes at $\mathrm{rt}$ ).

A $0.080 \times 0.050 \times 0.040 \mathrm{~mm}$ piece of a colorless crystal was mounted on a Cryoloop with Paratone oil. Data were collected in a nitrogen gas stream at 100(2) K using $\phi$ and $\varpi$ scans. Crystal-to-detector distance was $40 \mathrm{~mm}$ and exposure time was 60 seconds (depending on the $2 \theta$ range) per frame using a scan width 
of $1.00^{\circ}$. Data collection was $100.0 \%$ complete to $25.242^{\circ}$ in $\theta$. A total of 37545 reflections were collected covering the indices, $-14<=\mathrm{h}<=14,-24<=\mathrm{k}<=24,-13<=\mathrm{l}<=11.4311$ reflections were found to be symmetry independent, with a $\mathrm{R}_{\mathrm{int}}$ of 0.0702. Indexing and unit cell refinement indicated a Primitive, Monoclinic lattice. The space group was found to be $\boldsymbol{P 2}{ }_{1} / \boldsymbol{c}$. The data were integrated using the Bruker SAINT Software program and scaled using the SADABS software program. Solution by direct methods (SHELXT) produced a complete phasing model consistent with the proposed structure.

All nonhydrogen atoms were refined anisotropically by full-matrix least-squares (SHELXL-2014). All carbon bonded hydrogen atoms were placed using a riding model. Their positions were constrained relative to their parent atom using the appropriate HFIX command in SHELXL-2014. . Crystallographic data are summarized in Table 1.

Notes: Water molecule solvate

Table 20. Crystal data and structure refinement for Baran745.

Report date

Identification code

Empirical formula

Molecular formula

Formula weight

Temperature

Wavelength

Crystal system

Space group

Unit cell dimensions

Volume

Z

Density (calculated)

Absorption coefficient

$\mathrm{F}(000)$

Crystal size

Crystal color, habit

Theta range for data collection

Index ranges

Reflections collected

Independent reflections

Completeness to theta $=25.242^{\circ}$

Absorption correction

Max. and min. transmission

Refinement method

Data / restraints / parameters

Goodness-of-fit on $\mathrm{F}^{2}$

Final $R$ indices [I>2sigma(I)]

$\mathrm{R}$ indices (all data)

Largest diff. peak and hole
2019-10-17

baran745

C25 H31 N3 O2 Pt

C25 H29 N3 O Pt, H2 O

600.62

$100.0 \mathrm{~K}$

$0.71073 \AA$

Monoclinic

P 1 21/c 1

$\mathrm{a}=11.707(2) \AA \quad \alpha=90^{\circ}$.

$\mathrm{b}=19.744(4) \AA \quad \beta=117.839(4)^{\circ}$.

$\mathrm{c}=11.109(2) \AA \quad \gamma=90^{\circ}$.

2270.6(7) $\AA^{3}$

4

$1.757 \mathrm{Mg} / \mathrm{m}^{3}$

$6.206 \mathrm{~mm}^{-1}$

1184

$0.08 \times 0.05 \times 0.04 \mathrm{~mm}^{3}$

yellow block

1.967 to $25.681^{\circ}$.

$-14<=\mathrm{h}<=14,-24<=\mathrm{k}<=24,-13<=\mathrm{l}<=11$

37545

$4311[\mathrm{R}($ int $)=0.0702]$

$100.0 \%$

Semi-empirical from equivalents

0.7453 and 0.6667

Full-matrix least-squares on $\mathrm{F}^{2}$

4311 / 0 / 286

1.053

$\mathrm{R} 1=0.0361, \mathrm{wR} 2=0.0601$

$\mathrm{R} 1=0.0552, \mathrm{wR} 2=0.0643$

1.892 and -1.294 e. $\AA^{-3}$

Table 21. Atomic coordinates ( x 104) and equivalent isotropic displacement parameters $\left(\AA^{2} \times 10^{3}\right)$ for Baran745. $\mathrm{U}(\mathrm{eq})$ is defined as one third of the trace of the orthogonalized $\mathrm{U}^{\mathrm{ij}}$ tensor.

\begin{tabular}{lcccc}
\hline & $\mathrm{x}$ & $\mathrm{y}$ & $\mathrm{z}$ & $\mathrm{U}(\mathrm{eq})$ \\
\hline $\mathrm{Pt}(1)$ & $3993(1)$ & $5900(1)$ & $3347(1)$ & $17(1)$ \\
$\mathrm{O}(1)$ & $1653(4)$ & $5868(3)$ & $5271(4)$ & $44(1)$
\end{tabular}




\begin{tabular}{|c|c|c|c|c|}
\hline $\mathrm{N}(1)$ & $5583(4)$ & $6293(2)$ & $5058(4)$ & $17(1)$ \\
\hline $\mathrm{N}(2)$ & $3133(5)$ & $6120(2)$ & $4526(4)$ & $21(1)$ \\
\hline $\mathrm{N}(3)$ & $5072(4)$ & $5702(2)$ & $2385(5)$ & $20(1)$ \\
\hline $\mathrm{C}(1)$ & $6797(6)$ & $6342(3)$ & $5282(6)$ & $22(1)$ \\
\hline$C(2)$ & $7735(6)$ & $6686(3)$ & $6399(6)$ & $27(2)$ \\
\hline $\mathrm{C}(3)$ & $7422(6)$ & $6989(3)$ & $7300(6)$ & $29(2)$ \\
\hline $\mathrm{C}(4)$ & $6144(6)$ & $6939(3)$ & $7118(6)$ & $24(1)$ \\
\hline$C(5)$ & $5735(7)$ & $7226(3)$ & $8016(6)$ & $29(2)$ \\
\hline$C(6)$ & $4478(7)$ & $7168(3)$ & $7726(6)$ & $30(2)$ \\
\hline$C(7)$ & $3571(6)$ & $6807(3)$ & $6588(6)$ & $25(1)$ \\
\hline $\mathrm{C}(8)$ & $3930(5)$ & $6500(3)$ & $5693(5)$ & $18(1)$ \\
\hline $\mathrm{C}(9)$ & $5243(5)$ & $6581(3)$ & $5969(5)$ & $20(1)$ \\
\hline$C(10)$ & $2014(6)$ & $5842(4)$ & $4387(6)$ & $29(2)$ \\
\hline $\mathrm{C}(11)$ & 1191(6) & $5457(3)$ & $3112(6)$ & $27(2)$ \\
\hline$C(12)$ & $1133(5)$ & $5714(3)$ & $1779(6)$ & $21(1)$ \\
\hline $\mathrm{C}(13)$ & $2364(5)$ & $5532(3)$ & $1678(6)$ & $19(1)$ \\
\hline$C(14)$ & $2506(6)$ & $4757(3)$ & $1662(6)$ & $25(1)$ \\
\hline$C(15)$ & $2180(6)$ & $5798(3)$ & $308(5)$ & $21(1)$ \\
\hline$C(16)$ & $1910(6)$ & $6557(3)$ & $86(6)$ & $26(1)$ \\
\hline$C(17)$ & 711(6) & $6717(3)$ & 212(6) & $26(2)$ \\
\hline $\mathrm{C}(18)$ & $792(6)$ & $6478(3)$ & $1555(6)$ & $24(1)$ \\
\hline C(19) & $1795(7)$ & $6786(4)$ & $-1281(7)$ & $42(2)$ \\
\hline $\mathrm{C}(20)$ & $-458(6)$ & $6638(3)$ & $1606(7)$ & $35(2)$ \\
\hline $\mathrm{C}(21)$ & $5280(6)$ & $6176(3)$ & $1646(6)$ & $24(1)$ \\
\hline $\mathrm{C}(22)$ & $6124(6)$ & $6079(3)$ & $1129(6)$ & $31(2)$ \\
\hline $\mathrm{C}(23)$ & $6797(6)$ & $5477(3)$ & $1382(6)$ & $31(2)$ \\
\hline $\mathrm{C}(24)$ & $6568(6)$ & $4990(3)$ & $2117(6)$ & $30(2)$ \\
\hline$C(25)$ & $5717(6)$ & $5110(3)$ & $2615(6)$ & $26(1)$ \\
\hline $\mathrm{O}(1 \mathrm{~S})$ & $-910(5)$ & $5765(3)$ & $4787(7)$ & $66(2)$ \\
\hline
\end{tabular}

Table 22. Bond lengths $[\AA]$ and angles $\left[{ }^{\circ}\right]$ for Baran745.

\begin{tabular}{llll}
\hline $\mathrm{Pt}(1)-\mathrm{N}(1)$ & $2.092(4)$ & $\mathrm{C}(7)-\mathrm{H}(7)$ & 0.9500 \\
$\mathrm{Pt}(1)-\mathrm{N}(2)$ & $2.038(5)$ & $\mathrm{C}(7)-\mathrm{C}(8)$ & $1.387(8)$ \\
$\mathrm{Pt}(1)-\mathrm{N}(3)$ & $2.039(5)$ & $\mathrm{C}(8)-\mathrm{C}(9)$ & $1.429(8)$ \\
$\mathrm{Pt}(1)-\mathrm{C}(13)$ & $2.074(5)$ & $\mathrm{C}(10)-\mathrm{C}(11)$ & $1.497(8)$ \\
$\mathrm{O}(1)-\mathrm{C}(10)$ & $1.238(7)$ & $\mathrm{C}(11)-\mathrm{H}(11 \mathrm{~A})$ & 0.9900 \\
$\mathrm{~N}(1)-\mathrm{C}(1)$ & $1.327(7)$ & $\mathrm{C}(11)-\mathrm{H}(11 \mathrm{~B})$ & 0.9900 \\
$\mathrm{~N}(1)-\mathrm{C}(9)$ & $1.372(7)$ & $\mathrm{C}(11)-\mathrm{C}(12)$ & $1.537(8)$ \\
$\mathrm{N}(2)-\mathrm{C}(8)$ & $\mathrm{C}(12)-\mathrm{H}(12)$ & 1.0000 \\
$\mathrm{~N}(2)-\mathrm{C}(10)$ & $\mathrm{C}(12)-\mathrm{C}(13)$ & $1.538(8)$ \\
$\mathrm{N}(3)-\mathrm{C}(21)$ & $\mathrm{C}(12)-\mathrm{C}(18)$ & $1.550(8)$ \\
$\mathrm{N}(3)-\mathrm{C}(25)$ & $1.408(7)$ & $\mathrm{C}(13)-\mathrm{C}(14)$ & $1.541(8)$ \\
$\mathrm{C}(1)-\mathrm{H}(1)$ & $\mathrm{C}(13)-\mathrm{C}(15)$ & $1.528(8)$ \\
$\mathrm{C}(1)-\mathrm{C}(2)$ & $\mathrm{C}(14)-\mathrm{H}(14 \mathrm{~A})$ & 0.9800 \\
$\mathrm{C}(2)-\mathrm{H}(2)$ & $\mathrm{C}(14)-\mathrm{H}(14 \mathrm{~B})$ & 0.9800 \\
$\mathrm{C}(2)-\mathrm{C}(3)$ & $1.340(7)$ & $\mathrm{C}(14)-\mathrm{H}(14 \mathrm{C})$ & 0.9800 \\
$\mathrm{C}(3)-\mathrm{H}(3)$ & 0.9500 & $\mathrm{C}(15)-\mathrm{H}(15 \mathrm{~A})$ & 0.9900 \\
$\mathrm{C}(3)-\mathrm{C}(4)$ & $1.392(8)$ & $\mathrm{C}(15)-\mathrm{H}(15 \mathrm{~B})$ & 0.9900 \\
$\mathrm{C}(4)-\mathrm{C}(5)$ & 0.9500 & $\mathrm{C}(15)-\mathrm{C}(16)$ & $1.527(8)$ \\
$\mathrm{C}(4)-\mathrm{C}(9)$ & $1.355(9)$ & $\mathrm{C}(16)-\mathrm{H}(16)$ & 1.0000 \\
$\mathrm{C}(5)-\mathrm{H}(5)$ & 0.9500 & $\mathrm{C}(16)-\mathrm{C}(17)$ & $1.508(8)$ \\
$\mathrm{C}(5)-\mathrm{C}(6)$ & $1.417(9)$ & $\mathrm{C}(16)-\mathrm{C}(19)$ & $1.528(8)$ \\
$\mathrm{C}(6)-\mathrm{H}(6)$ & $1.411(9)$ & $\mathrm{C}(17)-\mathrm{H}(17 \mathrm{~A})$ & 0.9900 \\
$\mathrm{C}(6)-\mathrm{C}(7)$ & $1.408(8)$ & $\mathrm{C}(17)-\mathrm{H}(17 \mathrm{~B})$ & 0.9900
\end{tabular}




\begin{tabular}{|c|c|}
\hline $\mathrm{C}(17)-\mathrm{C}(18)$ & $1.525(8)$ \\
\hline C(18)-H(18) & 1.0000 \\
\hline C(18)-C(20) & $1.525(8)$ \\
\hline C(19)-H(19A) & 0.9800 \\
\hline C(19)-H(19B) & 0.9800 \\
\hline $\mathrm{C}(19)-\mathrm{H}(19 \mathrm{C})$ & 0.9800 \\
\hline $\mathrm{C}(20)-\mathrm{H}(20 \mathrm{~A})$ & 0.9800 \\
\hline $\mathrm{C}(20)-\mathrm{H}(20 \mathrm{~B})$ & 0.9800 \\
\hline C(20)-H(20C) & 0.9800 \\
\hline $\mathrm{C}(21)-\mathrm{H}(21)$ & 0.9500 \\
\hline $\mathrm{C}(21)-\mathrm{C}(22)$ & $1.368(8)$ \\
\hline $\mathrm{C}(22)-\mathrm{H}(22)$ & 0.9500 \\
\hline C(22)-C(23) & $1.379(9)$ \\
\hline $\mathrm{C}(23)-\mathrm{H}(23)$ & 0.9500 \\
\hline $\mathrm{C}(23)-\mathrm{C}(24)$ & $1.367(9)$ \\
\hline $\mathrm{C}(24)-\mathrm{H}(24)$ & 0.9500 \\
\hline C(24)-C(25) & $1.368(8)$ \\
\hline $\mathrm{C}(25)-\mathrm{H}(25)$ & 0.9500 \\
\hline $\mathrm{O}(1 \mathrm{~S})-\mathrm{H}(1 \mathrm{SA})$ & 0.8506 \\
\hline $\mathrm{O}(1 \mathrm{~S})-\mathrm{H}(1 \mathrm{SB})$ & 0.8502 \\
\hline $\mathrm{N}(2)-\mathrm{Pt}(1)-\mathrm{N}(1)$ & $80.44(18)$ \\
\hline $\mathrm{N}(2)-\mathrm{Pt}(1)-\mathrm{N}(3)$ & $172.66(18)$ \\
\hline $\mathrm{N}(2)-\mathrm{Pt}(1)-\mathrm{C}(13)$ & $97.2(2)$ \\
\hline $\mathrm{N}(3)-\mathrm{Pt}(1)-\mathrm{N}(1)$ & $92.37(18)$ \\
\hline $\mathrm{N}(3)-\mathrm{Pt}(1)-\mathrm{C}(13)$ & $90.0(2)$ \\
\hline $\mathrm{C}(13)-\mathrm{Pt}(1)-\mathrm{N}(1)$ & $177.5(2)$ \\
\hline C(1)-N(1)-Pt(1) & 128.1(4) \\
\hline C(1)-N(1)-C(9) & 119.1(5) \\
\hline $\mathrm{C}(9)-\mathrm{N}(1)-\mathrm{Pt}(1)$ & $112.5(4)$ \\
\hline $\mathrm{C}(8)-\mathrm{N}(2)-\mathrm{Pt}(1)$ & $113.0(4)$ \\
\hline $\mathrm{C}(10)-\mathrm{N}(2)-\mathrm{Pt}(1)$ & $125.4(4)$ \\
\hline $\mathrm{C}(10)-\mathrm{N}(2)-\mathrm{C}(8)$ & $120.6(5)$ \\
\hline $\mathrm{C}(21)-\mathrm{N}(3)-\mathrm{Pt}(1)$ & $121.4(4)$ \\
\hline $\mathrm{C}(21)-\mathrm{N}(3)-\mathrm{C}(25)$ & $118.4(5)$ \\
\hline C(25)-N(3)-Pt(1) & 119.7(4) \\
\hline $\mathrm{N}(1)-\mathrm{C}(1)-\mathrm{H}(1)$ & 118.9 \\
\hline $\mathrm{N}(1)-\mathrm{C}(1)-\mathrm{C}(2)$ & $122.2(6)$ \\
\hline $\mathrm{C}(2)-\mathrm{C}(1)-\mathrm{H}(1)$ & 118.9 \\
\hline $\mathrm{C}(1)-\mathrm{C}(2)-\mathrm{H}(2)$ & 119.9 \\
\hline$C(3)-C(2)-C(1)$ & $120.1(6)$ \\
\hline $\mathrm{C}(3)-\mathrm{C}(2)-\mathrm{H}(2)$ & 119.9 \\
\hline $\mathrm{C}(2)-\mathrm{C}(3)-\mathrm{H}(3)$ & 120.1 \\
\hline $\mathrm{C}(2)-\mathrm{C}(3)-\mathrm{C}(4)$ & 119.7(6) \\
\hline $\mathrm{C}(4)-\mathrm{C}(3)-\mathrm{H}(3)$ & 120.1 \\
\hline$C(5)-C(4)-C(3)$ & $123.7(6)$ \\
\hline $\mathrm{C}(9)-\mathrm{C}(4)-\mathrm{C}(3)$ & $117.3(6)$ \\
\hline$C(9)-C(4)-C(5)$ & $119.0(6)$ \\
\hline $\mathrm{C}(4)-\mathrm{C}(5)-\mathrm{H}(5)$ & 120.4 \\
\hline$C(6)-C(5)-C(4)$ & 119.1(6) \\
\hline $\mathrm{C}(6)-\mathrm{C}(5)-\mathrm{H}(5)$ & 120.4 \\
\hline $\mathrm{C}(5)-\mathrm{C}(6)-\mathrm{H}(6)$ & 118.8 \\
\hline $\mathrm{C}(5)-\mathrm{C}(6)-\mathrm{C}(7)$ & $122.4(6)$ \\
\hline $\mathrm{C}(7)-\mathrm{C}(6)-\mathrm{H}(6)$ & 118.8 \\
\hline $\mathrm{C}(6)-\mathrm{C}(7)-\mathrm{H}(7)$ & 119.6 \\
\hline$C(8)-C(7)-C(6)$ & $120.8(6)$ \\
\hline
\end{tabular}

$\begin{array}{ll} & \\ \mathrm{C}(8)-\mathrm{C}(7)-\mathrm{H}(7) & 119.6 \\ \mathrm{~N}(2)-\mathrm{C}(8)-\mathrm{C}(9) & 116.2(5) \\ \mathrm{C}(7)-\mathrm{C}(8)-\mathrm{N}(2) & 126.9(5) \\ \mathrm{C}(7)-\mathrm{C}(8)-\mathrm{C}(9) & 116.9(5) \\ \mathrm{N}(1)-\mathrm{C}(9)-\mathrm{C}(4) & 121.6(5) \\ \mathrm{N}(1)-\mathrm{C}(9)-\mathrm{C}(8) & 116.7(5) \\ \mathrm{C}(4)-\mathrm{C}(9)-\mathrm{C}(8) & 121.8(5) \\ \mathrm{O}(1)-\mathrm{C}(10)-\mathrm{N}(2) & 124.1(6) \\ \mathrm{O}(1)-\mathrm{C}(10)-\mathrm{C}(11) & 117.6(5) \\ \mathrm{N}(2)-\mathrm{C}(10)-\mathrm{C}(11) & 118.2(5) \\ \mathrm{C}(10)-\mathrm{C}(11)-\mathrm{H}(11 \mathrm{~A}) & 107.9 \\ \mathrm{C}(10)-\mathrm{C}(11)-\mathrm{H}(11 \mathrm{~B}) & 107.9 \\ \mathrm{C}(10)-\mathrm{C}(11)-\mathrm{C}(12) & 117.6(5) \\ \mathrm{H}(11 \mathrm{~A})-\mathrm{C}(11)-\mathrm{H}(11 \mathrm{~B}) & 107.2 \\ \mathrm{C}(12)-\mathrm{C}(11)-\mathrm{H}(11 \mathrm{~A}) & 107.9 \\ \mathrm{C}(12)-\mathrm{C}(11)-\mathrm{H}(11 \mathrm{~B}) & 107.9 \\ \mathrm{C}(11)-\mathrm{C}(12)-\mathrm{H}(12) & 106.6 \\ \mathrm{C}(11)-\mathrm{C}(12)-\mathrm{C}(13) & 111.8(5) \\ \mathrm{C}(11)-\mathrm{C}(12)-\mathrm{C}(18) & 111.6(5) \\ \mathrm{C}(13)-\mathrm{C}(12)-\mathrm{H}(12) & 106.6 \\ \mathrm{C}(13)-\mathrm{C}(12)-\mathrm{C}(18) & 113.2(5) \\ \mathrm{C}(18)-\mathrm{C}(12)-\mathrm{H}(12) & 106.6 \\ \mathrm{C}(12)-\mathrm{C}(13)-\mathrm{Pt}(1) & 110.8(4) \\ \mathrm{C}(12)-\mathrm{C}(13)-\mathrm{C}(14) & 110.0(5) \\ \mathrm{C}(14)-\mathrm{C}(13)-\mathrm{Pt}(1) & 107.4(4) \\ \mathrm{C}(15)-\mathrm{C}(13)-\mathrm{Pt}(1) & 114.4(4) \\ \mathrm{C}(15)-\mathrm{C}(13)-\mathrm{C}(12) & 106.9(5) \\ \mathrm{C}(15)-\mathrm{C}(13)-\mathrm{C}(14) & 107.2(5) \\ \mathrm{C}(13)-\mathrm{C}(14)-\mathrm{H}(14 \mathrm{~A}) & 109.5 \\ \mathrm{C}(13)-\mathrm{C}(14)-\mathrm{H}(14 \mathrm{~B}) & 109.5 \\ \mathrm{C}(13)-\mathrm{C}(14)-\mathrm{H}(14 \mathrm{C}) & 109.5 \\ \mathrm{H}(14 \mathrm{~A})-\mathrm{C}(14)-\mathrm{H}(14 \mathrm{~B}) & 109.5 \\ \mathrm{H}(14 \mathrm{~A})-\mathrm{C}(14)-\mathrm{H}(14 \mathrm{C}) & 109.5 \\ \mathrm{H}(14 \mathrm{~B})-\mathrm{C}(14)-\mathrm{H}(14 \mathrm{C}) & 109.5 \\ \mathrm{C}(13)-\mathrm{C}(15)-\mathrm{H}(15 \mathrm{~A}) & 108.6 \\ \mathrm{C}(13)-\mathrm{C}(15)-\mathrm{H}(15 \mathrm{~B}) & 108.6 \\ \mathrm{H}(15 \mathrm{~A})-\mathrm{C}(15)-\mathrm{H}(15 \mathrm{~B}) & 107.5 \\ \mathrm{C}(16)-\mathrm{C}(15)-\mathrm{C}(13) & 114.8(5) \\ \mathrm{C}(16)-\mathrm{C}(15)-\mathrm{H}(15 \mathrm{~A}) & 108.6 \\ \mathrm{C}(16)-\mathrm{C}(15)-\mathrm{H}(15 \mathrm{~B}) & 108.6 \\ \mathrm{C}(15)-\mathrm{C}(16)-\mathrm{H}(16) & 108.1 \\ \mathrm{C}(15)-\mathrm{C}(16)-\mathrm{C}(19) & 111.4(5) \\ \mathrm{C}(17)-\mathrm{C}(16)-\mathrm{C}(15) & 108.8(5) \\ \mathrm{C}(17)-\mathrm{C}(16)-\mathrm{H}(16) & 108.1 \\ \mathrm{C}(17)-\mathrm{C}(16)-\mathrm{C}(19) & 112.1(5) \\ \mathrm{C}(19)-\mathrm{C}(16)-\mathrm{H}(16) & 108.1 \\ \mathrm{C}(16)-\mathrm{C}(17)-\mathrm{H}(17 \mathrm{~A}) & 109.0 \\ \mathrm{C}(16)-\mathrm{C}(17)-\mathrm{H}(17 \mathrm{~B}) & 109.0 \\ \mathrm{C}(16)-\mathrm{C}(17)-\mathrm{C}(18) & 113.0(5) \\ \mathrm{H}(17 \mathrm{~A})-\mathrm{C}(17)-\mathrm{H}(17 \mathrm{~B}) & 107.8 \\ \mathrm{C}(18)-\mathrm{C}(17)-\mathrm{H}(17 \mathrm{~A}) & 109.0 \\ \mathrm{C}(18)-\mathrm{C}(17)-\mathrm{H}(17 \mathrm{~B}) & 109.0 \\ \mathrm{C}(12)-\mathrm{C}(18)-\mathrm{H}(18) & 107.8 \\ \mathrm{C}(17)-\mathrm{C}(18)-\mathrm{C}(12) & 110.6(5) \\ \mathrm{C}(17)-\mathrm{C}(18)-\mathrm{H}(18) & 107.8 \\ \mathrm{C}(20)-\mathrm{C}(18)-\mathrm{C}(12) & 112.1(5) \\ & \\ & \end{array}$




$\begin{array}{llll}\mathrm{C}(20)-\mathrm{C}(18)-\mathrm{C}(17) & 110.5(5) & \mathrm{N}(3)-\mathrm{C}(21)-\mathrm{C}(22) & 122.1(6) \\ \mathrm{C}(20)-\mathrm{C}(18)-\mathrm{H}(18) & 107.8 & \mathrm{C}(22)-\mathrm{C}(21)-\mathrm{H}(21) & 118.9 \\ \mathrm{C}(16)-\mathrm{C}(19)-\mathrm{H}(19 \mathrm{~A}) & 109.5 & \mathrm{C}(21)-\mathrm{C}(22)-\mathrm{H}(22) & 120.3 \\ \mathrm{C}(16)-\mathrm{C}(19)-\mathrm{H}(19 \mathrm{~B}) & 109.5 & \mathrm{C}(21)-\mathrm{C}(22)-\mathrm{C}(23) & 119.4(6) \\ \mathrm{C}(16)-\mathrm{C}(19)-\mathrm{H}(19 \mathrm{C}) & 109.5 & \mathrm{C}(23)-\mathrm{C}(22)-\mathrm{H}(22) & 120.3 \\ \mathrm{H}(19 \mathrm{~A})-\mathrm{C}(19)-\mathrm{H}(19 \mathrm{~B}) & 109.5 & \mathrm{C}(22)-\mathrm{C}(23)-\mathrm{H}(23) & 120.8 \\ \mathrm{H}(19 \mathrm{~A})-\mathrm{C}(19)-\mathrm{H}(19 \mathrm{C}) & 109.5 & \mathrm{C}(24)-\mathrm{C}(23)-\mathrm{C}(22) & 118.4(6) \\ \mathrm{H}(19 \mathrm{~B})-\mathrm{C}(19)-\mathrm{H}(19 \mathrm{C}) & 109.5 & \mathrm{C}(24)-\mathrm{C}(23)-\mathrm{H}(23) & 120.8 \\ \mathrm{C}(18)-\mathrm{C}(20)-\mathrm{H}(20 \mathrm{~A}) & 109.5 & \mathrm{C}(23)-\mathrm{C}(24)-\mathrm{H}(24) & 119.9 \\ \mathrm{C}(18)-\mathrm{C}(20)-\mathrm{H}(20 \mathrm{~B}) & 109.5 & \mathrm{C}(23)-\mathrm{C}(24)-\mathrm{C}(25) & 120.2(6) \\ \mathrm{C}(18)-\mathrm{C}(20)-\mathrm{H}(20 \mathrm{C}) & 109.5 & \mathrm{C}(25)-\mathrm{C}(24)-\mathrm{H}(24) & 119.9 \\ \mathrm{H}(20 \mathrm{~A})-\mathrm{C}(20)-\mathrm{H}(20 \mathrm{~B}) & 109.5 & \mathrm{~N}(3)-\mathrm{C}(25)-\mathrm{C}(24) & 121.4(6) \\ \mathrm{H}(20 \mathrm{~A})-\mathrm{C}(20)-\mathrm{H}(20 \mathrm{C}) & 109.5 & \mathrm{~N}(3)-\mathrm{C}(25)-\mathrm{H}(25) & 119.3 \\ \mathrm{H}(20 \mathrm{~B})-\mathrm{C}(20)-\mathrm{H}(20 \mathrm{C}) & 109.5 & \mathrm{C}(24)-\mathrm{C}(25)-\mathrm{H}(25) & 119.3 \\ \mathrm{~N}(3)-\mathrm{C}(21)-\mathrm{H}(21) & 118.9 & \mathrm{H}(1 \mathrm{SA})-\mathrm{O}(1 \mathrm{~S})-\mathrm{H}(1 \mathrm{SB}) & 109.4 \\ & & & \end{array}$

Table 23. Anisotropic displacement parameters $\left(\AA^{2} \times 10^{3}\right)$ for Baran745. The anisotropic displacement factor exponent takes the form: $-2 \pi^{2}\left[h^{2} a^{* 2} U^{11}+\ldots+2 h k^{*} b^{*} U^{12}\right]$

\begin{tabular}{|c|c|c|c|c|c|c|}
\hline & $\mathrm{U}^{11}$ & $\mathrm{U}^{22}$ & $\mathrm{U}^{33}$ & $\mathrm{U}^{23}$ & $\mathrm{U}^{13}$ & $\mathrm{U}^{12}$ \\
\hline $\mathrm{Pt}(1)$ & $15(1)$ & $18(1)$ & $16(1)$ & $0(1)$ & $6(1)$ & $-2(1)$ \\
\hline $\mathrm{O}(1)$ & $25(2)$ & $88(4)$ & $26(2)$ & $-9(3)$ & $16(2)$ & $-19(3)$ \\
\hline $\mathrm{N}(1)$ & $18(3)$ & $15(3)$ & $17(2)$ & 1(2) & $5(2)$ & $-3(2)$ \\
\hline $\mathrm{N}(2)$ & 24(3) & 24(3) & $16(2)$ & $3(2)$ & $9(2)$ & $0(2)$ \\
\hline $\mathrm{N}(3)$ & $18(3)$ & $22(3)$ & 19(3) & $-2(2)$ & $7(2)$ & $0(2)$ \\
\hline $\mathrm{C}(1)$ & $19(3)$ & $18(3)$ & $23(3)$ & $9(3)$ & $4(3)$ & $0(3)$ \\
\hline$C(2)$ & $13(3)$ & $26(4)$ & $32(4)$ & 11(3) & $2(3)$ & $-1(3)$ \\
\hline$C(3)$ & 32(4) & $15(3)$ & 23(3) & $5(3)$ & $-1(3)$ & $-6(3)$ \\
\hline $\mathrm{C}(4)$ & $27(4)$ & $15(3)$ & $18(3)$ & $5(2)$ & $1(3)$ & $-2(3)$ \\
\hline$C(5)$ & $45(4)$ & $15(3)$ & $16(3)$ & $0(3)$ & $6(3)$ & $-1(3)$ \\
\hline$C(6)$ & $51(5)$ & $17(3)$ & $26(3)$ & 4(3) & 21(3) & $7(3)$ \\
\hline$C(7)$ & $32(4)$ & 22(3) & 24(3) & 4(3) & $15(3)$ & $3(3)$ \\
\hline $\mathrm{C}(8)$ & $19(3)$ & $17(3)$ & $12(3)$ & $4(2)$ & $3(2)$ & 2(2) \\
\hline C(9) & $21(3)$ & $16(3)$ & 14(3) & $8(2)$ & $2(3)$ & $4(3)$ \\
\hline $\mathrm{C}(10)$ & 19(3) & $46(4)$ & 25(3) & $2(3)$ & 11(3) & $-1(3)$ \\
\hline $\mathrm{C}(11)$ & 20(3) & $36(4)$ & $28(3)$ & $-2(3)$ & 13(3) & $-4(3)$ \\
\hline$C(12)$ & $13(3)$ & $23(3)$ & 21(3) & $-6(3)$ & $4(2)$ & $-5(3)$ \\
\hline $\mathrm{C}(13)$ & $18(3)$ & $15(3)$ & 20(3) & $-6(3)$ & 7(3) & $-7(3)$ \\
\hline $\mathrm{C}(14)$ & $26(4)$ & 20(3) & $26(3)$ & $-4(3)$ & $8(3)$ & $-5(3)$ \\
\hline$C(15)$ & $19(3)$ & $23(3)$ & $18(3)$ & $-4(3)$ & $5(3)$ & $-4(3)$ \\
\hline$C(16)$ & $24(3)$ & $24(3)$ & $24(3)$ & $4(3)$ & $5(3)$ & $-3(3)$ \\
\hline $\mathrm{C}(17)$ & 20(3) & 20(3) & $26(3)$ & $-1(3)$ & 1(3) & $7(3)$ \\
\hline $\mathrm{C}(18)$ & $18(4)$ & $22(3)$ & 23(3) & $-8(3)$ & $3(3)$ & 2(3) \\
\hline$C(19)$ & $43(5)$ & $47(5)$ & $37(4)$ & 19(4) & $18(4)$ & 4(4) \\
\hline $\mathrm{C}(20)$ & $24(4)$ & $42(4)$ & $34(4)$ & $-10(3)$ & $9(3)$ & $8(3)$ \\
\hline$C(21)$ & 23(4) & 22(3) & 23(3) & $-4(3)$ & $9(3)$ & $-7(3)$ \\
\hline$C(22)$ & 28(4) & 41(4) & $28(4)$ & $-4(3)$ & $15(3)$ & $-7(3)$ \\
\hline $\mathrm{C}(23)$ & $31(4)$ & $36(4)$ & $33(4)$ & $-7(3)$ & 21(3) & $-3(3)$ \\
\hline$C(24)$ & 27(4) & 28(4) & $36(4)$ & $-7(3)$ & $16(3)$ & $4(3)$ \\
\hline$C(25)$ & $27(4)$ & 21(3) & 30(4) & $3(3)$ & $14(3)$ & 3(3) \\
\hline $\mathrm{O}(1 \mathrm{~S})$ & $45(3)$ & $84(5)$ & $81(4)$ & $9(4)$ & $41(3)$ & $4(3)$ \\
\hline
\end{tabular}


Table 24. Hydrogen coordinates ( x $\left.10^{4}\right)$ and isotropic displacement parameters $\left(\AA^{2} \times 10^{3}\right)$ for Baran745.

\begin{tabular}{|c|c|c|c|c|}
\hline & $\mathrm{x}$ & $\mathrm{y}$ & Z & $\mathrm{U}(\mathrm{eq})$ \\
\hline $\mathrm{H}(1)$ & 7033 & 6134 & 4658 & 27 \\
\hline $\mathrm{H}(2)$ & 8596 & 6709 & 6530 & 32 \\
\hline $\mathrm{H}(3)$ & 8057 & 7234 & 8051 & 34 \\
\hline $\mathrm{H}(5)$ & 6332 & 7457 & 8812 & 35 \\
\hline $\mathrm{H}(6)$ & 4200 & 7378 & 8314 & 36 \\
\hline $\mathrm{H}(7)$ & 2703 & 6773 & 6431 & 30 \\
\hline $\mathrm{H}(11 \mathrm{~A})$ & 299 & 5449 & 2993 & 32 \\
\hline $\mathrm{H}(11 \mathrm{~B})$ & 1502 & 4983 & 3249 & 32 \\
\hline $\mathrm{H}(12)$ & 406 & 5467 & 1023 & 25 \\
\hline $\mathrm{H}(14 \mathrm{~A})$ & 3203 & 4646 & 1443 & 38 \\
\hline $\mathrm{H}(14 \mathrm{~B})$ & 1694 & 4560 & 974 & 38 \\
\hline $\mathrm{H}(14 \mathrm{C})$ & 2710 & 4573 & 2559 & 38 \\
\hline $\mathrm{H}(15 \mathrm{~A})$ & 2968 & 5695 & 225 & 26 \\
\hline $\mathrm{H}(15 \mathrm{~B})$ & 1453 & 5551 & -427 & 26 \\
\hline $\mathrm{H}(16)$ & 2652 & 6804 & 825 & 32 \\
\hline $\mathrm{H}(17 \mathrm{~A})$ & -42 & 6500 & -550 & 31 \\
\hline $\mathrm{H}(17 \mathrm{~B})$ & 566 & 7213 & 131 & 31 \\
\hline $\mathrm{H}(18)$ & 1506 & 6734 & 2306 & 28 \\
\hline $\mathrm{H}(19 \mathrm{~A})$ & 2616 & 6705 & -1292 & 63 \\
\hline $\mathrm{H}(19 \mathrm{~B})$ & 1588 & 7270 & -1409 & 63 \\
\hline $\mathrm{H}(19 \mathrm{C})$ & 1108 & 6528 & -2017 & 63 \\
\hline $\mathrm{H}(20 \mathrm{~A})$ & -1157 & 6355 & 945 & 53 \\
\hline $\mathrm{H}(20 \mathrm{~B})$ & -676 & 7117 & 1385 & 53 \\
\hline $\mathrm{H}(20 \mathrm{C})$ & -344 & 6546 & 2523 & 53 \\
\hline $\mathrm{H}(21)$ & 4827 & 6593 & 1475 & 28 \\
\hline $\mathrm{H}(22)$ & 6246 & 6422 & 601 & 38 \\
\hline $\mathrm{H}(23)$ & 7405 & 5404 & 1054 & 37 \\
\hline $\mathrm{H}(24)$ & 7002 & 4567 & 2283 & 36 \\
\hline $\mathrm{H}(25)$ & 5576 & 4768 & 3135 & 31 \\
\hline $\mathrm{H}(1 \mathrm{SA})$ & -941 & 5338 & 4874 & 98 \\
\hline $\mathrm{H}(1 \mathrm{SB})$ & -157 & 5877 & 4926 & 98 \\
\hline
\end{tabular}




\section{Compound SI-2}<smiles>C[C@@H]1C[C@H]([SiH3])[C@H](CCS(=O)(=O)c2ccccc2)[C@H](C(O)CCS(=O)(=O)c2ccccc2)C1</smiles>

${ }^{1} \mathbf{H}$ NMR (600 MHz, C6D6): $\delta 7.91(\mathrm{dd}, J=7.5,2.1 \mathrm{~Hz}, 2 \mathrm{H}), 7.84(\mathrm{dd}, J=7.7,1.8 \mathrm{~Hz}, 2 \mathrm{H}), 7.00$ $-6.94(\mathrm{~m}, 6 \mathrm{H}), 3.57(\mathrm{~d}, J=9.0 \mathrm{~Hz}, 1 \mathrm{H}), 2.99(\mathrm{ddd}, J=14.4,9.0,5.8 \mathrm{~Hz}, 1 \mathrm{H}), 2.95-2.84(\mathrm{~m}$, 2H), 2.80 (ddd, $J=14.2,8.8,6.2 \mathrm{~Hz}, 1 \mathrm{H}), 1.91-1.87$ (m, 2H), 1.84 (ddt, $J=14.7,9.1,4.6 \mathrm{~Hz}$, $1 \mathrm{H}), 1.62-1.55(\mathrm{~m}, 1 \mathrm{H}), 1.50(\mathrm{~s}, 1 \mathrm{H}), 1.38-1.30(\mathrm{~m}, 1 \mathrm{H}), 1.28(\mathrm{~d}, J=9.1 \mathrm{~Hz}, 1 \mathrm{H}), 1.07$ (dd, $J$ $=9.6,4.6 \mathrm{~Hz}, 1 \mathrm{H}), 0.98-0.90(\mathrm{~m}, 1 \mathrm{H}), 0.78(\mathrm{~d}, J=6.5 \mathrm{~Hz}, 4 \mathrm{H}), 0.71(\mathrm{t}, J=11.5 \mathrm{~Hz}, 1 \mathrm{H}), 0.68$ $-0.63(\mathrm{~m}, 1 \mathrm{H}), 0.61(\mathrm{~d}, J=6.5 \mathrm{~Hz}, 3 \mathrm{H}), 0.40(\mathrm{q}, J=12.0 \mathrm{~Hz}, 1 \mathrm{H})$.

${ }^{13}$ C NMR (151 MHz, C6D6): $\delta$ 140.6, 140.3, 133.3, 133.3, 129.3, 129.3, 128.4, 69.2, 54.3, 52.7, $44.3,44.3,42.4,35.0,33.2,31.8,28.2,22.8,21.2,20.1$.

\section{C-H Activation Product Inhibition Study}
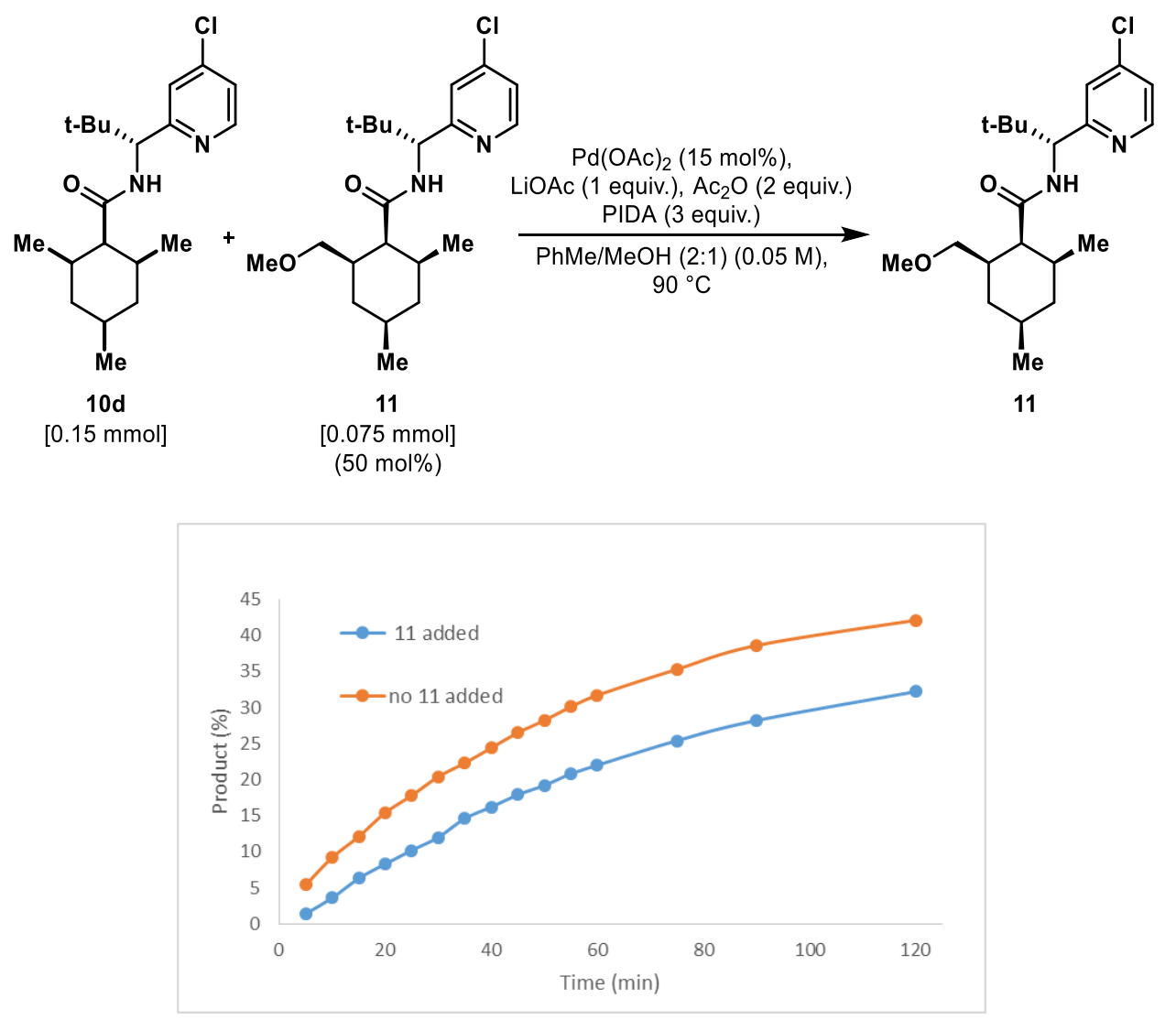

Figure 1. Effect of added 11 on product formation over time. 


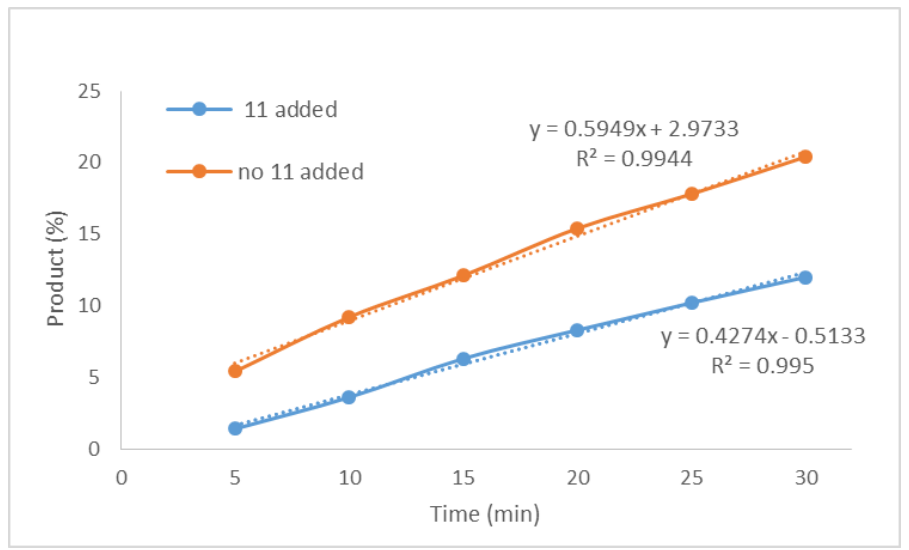

Figure 2. Effect of added 11 on initial rate.

- Data is suggestive of product inhibition.

Methods: Experiments were performed on an Unchained Labs (Pleasanton, CA, USA) Freeslate Junior system equipped with an OSR module (overhead-stirred pressure reactors with sampling capabilities) and using small volume stainless steel adapters that hold 12x75 mm test tubes.

\section{REFERENCES}

(1) Snider, B. B.; Qing, Lu. Total Synthesis of (.t-.)-Pyridoxatin. J. Org. Chem. 1994, 59, 80658070 .

(2) Primer, D. N.; Molander, G. A. Enabling the Cross-Coupling of Tertiary Organoboron Nucleophiles through Radical-Mediated Alkyl Transfer. Journal of the American Chemical Society 2017, 139, 9847-9850.

(3) Hirano, K.; Miura, M. A lesson for site-selective C-H functionalization on 2-pyridones: radical, organometallic, directing group and steric controls. Chemical Science 2018, 9, 22-32.

(4) Johnson, J. A.; Sames, D. C-H Bond Activation of Hydrocarbon Segments in Complex Organic Molecules: Total Synthesis of the Antimitotic Rhazinilam. Journal of the American Chemical Society 2000, 122, 6321-6322.

(5) Le Bras, J.; Muzart, J. C-O Bonds from Pd-Catalyzed C(sp3)-H Reactions Mediated by Heteroatomic Groups. Eur. J. Org. Chem., 2018, 1176-1203.

(6) Collados, J. F.; Toledano, E.; Guijarro, D.; Yus, M. Microwave-Assisted Solvent-Free Synthesis of Enantiomerically Pure N-(tert-Butylsulfinyl)imines. The Journal of Organic Chemistry 2012, 77, 5744-5750.

(7) Kim, Y.; Kim, S.-T.; Kang, D.; Sohn, T.-i.; Jang, E.; Baik, M.-H.; Hong, S. Stereoselective construction of sterically hindered oxaspirocycles via chiral bidentate directing group-mediated C(sp3)-O bond formation. Chemical Science 2018, 9, 1473-1480. 
(8) Li, G.; Xu, X.; Tian, H.; Liu, X.; Chen, W.; Yang, X.; Zhang, H. Asymmetric synthesis of $\delta$ amino acid derivatives via diastereoselective vinylogous Mannich reactions between N-tertbutanesulfinyl imines and dioxinone-derived lithium dienolate. RSC Advances 2017, 7, 5082250828.

(9) Youssef Bennani, S. C., Sanjay Chemburkar, Jinhua Chen, Michael Dart, Ashok Gupta, Lei Wang Cycloalkylamides and their therapeutic applications. US20040209858A1, 2003.

(10) Bian, J.; Blakemore, D.; Warmus, J. S.; Sun, J.; Corbett, M.; Rose, C. R.; Bechle, B. M. Diastereoselective Synthesis of $\beta$-Heteroaryl syn- $\alpha$-Methyl- $\beta$-Amino Acid Derivatives via a Double Chiral Auxiliary Approach. Organic Letters 2013, 15, 562-565.

(11) Novák, P.; Correa, A.; Gallardo-Donaire, J.; Martin, R. Synergistic Palladium-Catalyzed $\mathrm{C}(\mathrm{sp} 3) \square \mathrm{H}$ Activation/C(sp3) $\square \mathrm{O}$ Bond Formation: A Direct, Step-Economical Route to Benzolactones. Angewandte Chemie International Edition 2011, 50, 12236-12239.

(12) Boucher, M. M.; Furigay, M. H.; Quach, P. K.; Brindle, C. S. Liquid-Liquid Extraction Protocol for the Removal of Aldehydes and Highly Reactive Ketones from Mixtures. Organic Process Research \& Development 2017, 21, 1394-1403.

(13) Mizuki, K.; Iwahashi, K.; Murata, N.; Ikeda, M.; Nakai, Y.; Yoneyama, H.; Harusawa, S.; Usami, Y. Synthesis of Marine Natural Product (-)-Pericosine E. Organic Letters 2014, 16, 37603763.

(14) (a) Du, L.; Robles, A. J.; King, J. B.; Powell, D. R.; Miller, A. N.; Mooberry, S. L.; Cichewicz, R. H. Crowdsourcing Natural Products Discovery to Access Uncharted Dimensions of Fungal Metabolite Diversity. Angewandte Chemie International Edition 2014, 53, 804-809. (b) Du, L.; You, J.; Nicholas, K. M.; Cichewicz, R. H. Chemoreactive Natural Products that Afford Resistance Against Disparate Antibiotics and Toxins. Angewandte Chemie International Edition 2016, 55, 4220-4225. 


\section{NMR SPECTRA}

\section{Compound $7{ }^{1} \mathrm{H}$ NMR}

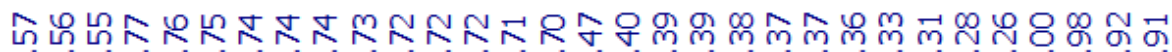

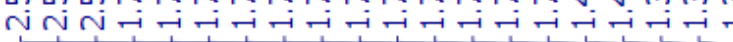

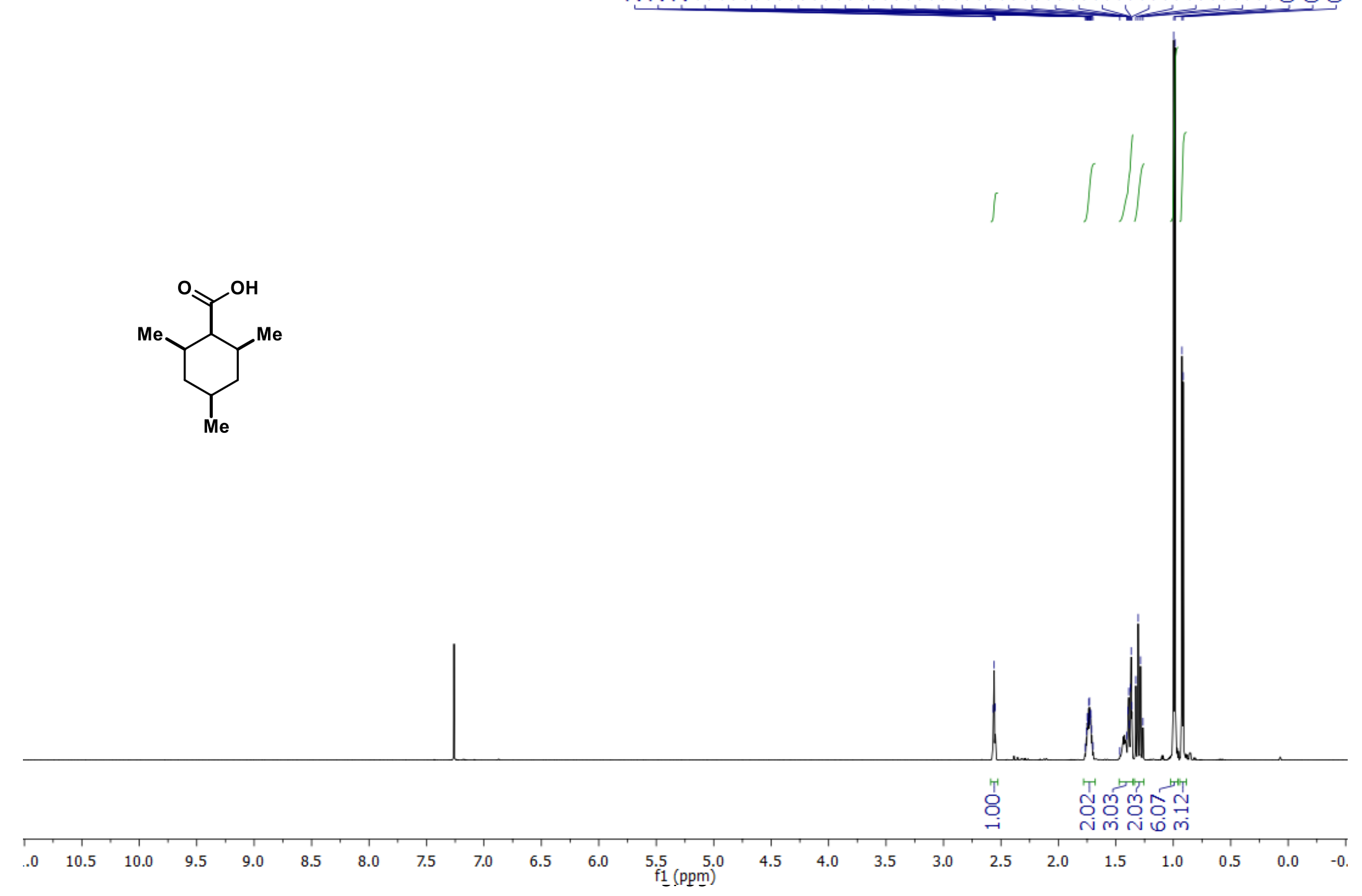


Compound $7{ }^{13} \mathrm{C}$ NMR

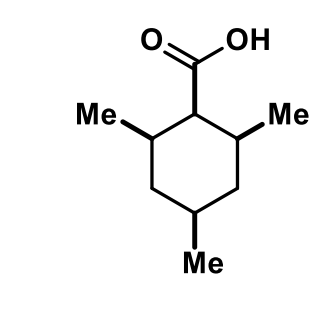

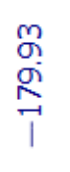

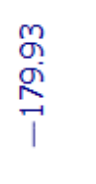

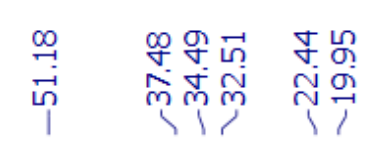




\section{Compound SI-9 ${ }^{1}$ H NMR}

芯家员

$\stackrel{\stackrel{D}{i}}{i}$

$\overbrace{\substack{n-t-B u \\ 11}}^{c l}$

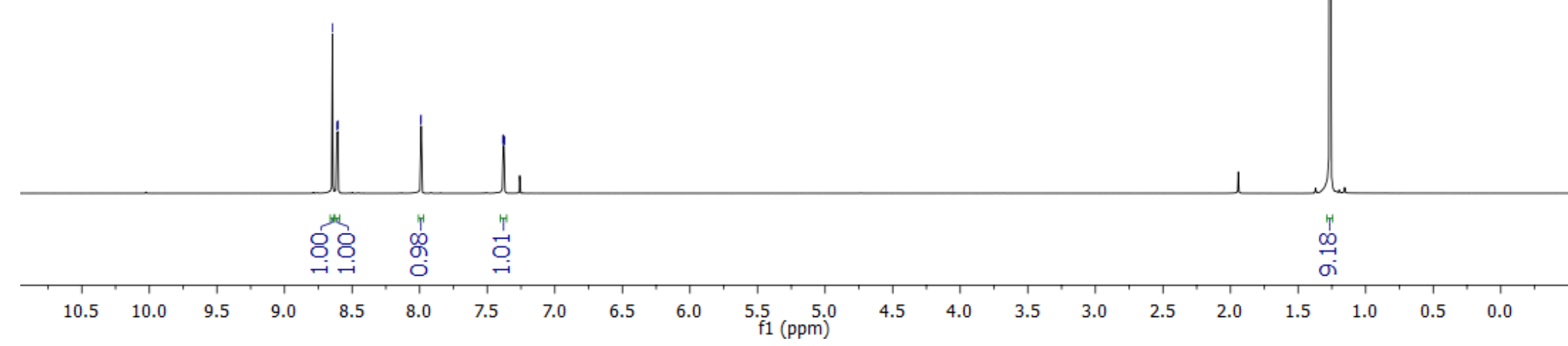

SI-101 


\section{Compound SI-9 ${ }^{13} \mathrm{C}$ NMR}

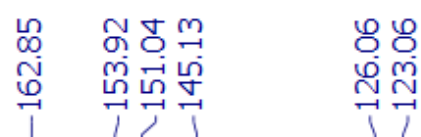

$\stackrel{\infty}{\infty} \underset{\infty}{\infty}$
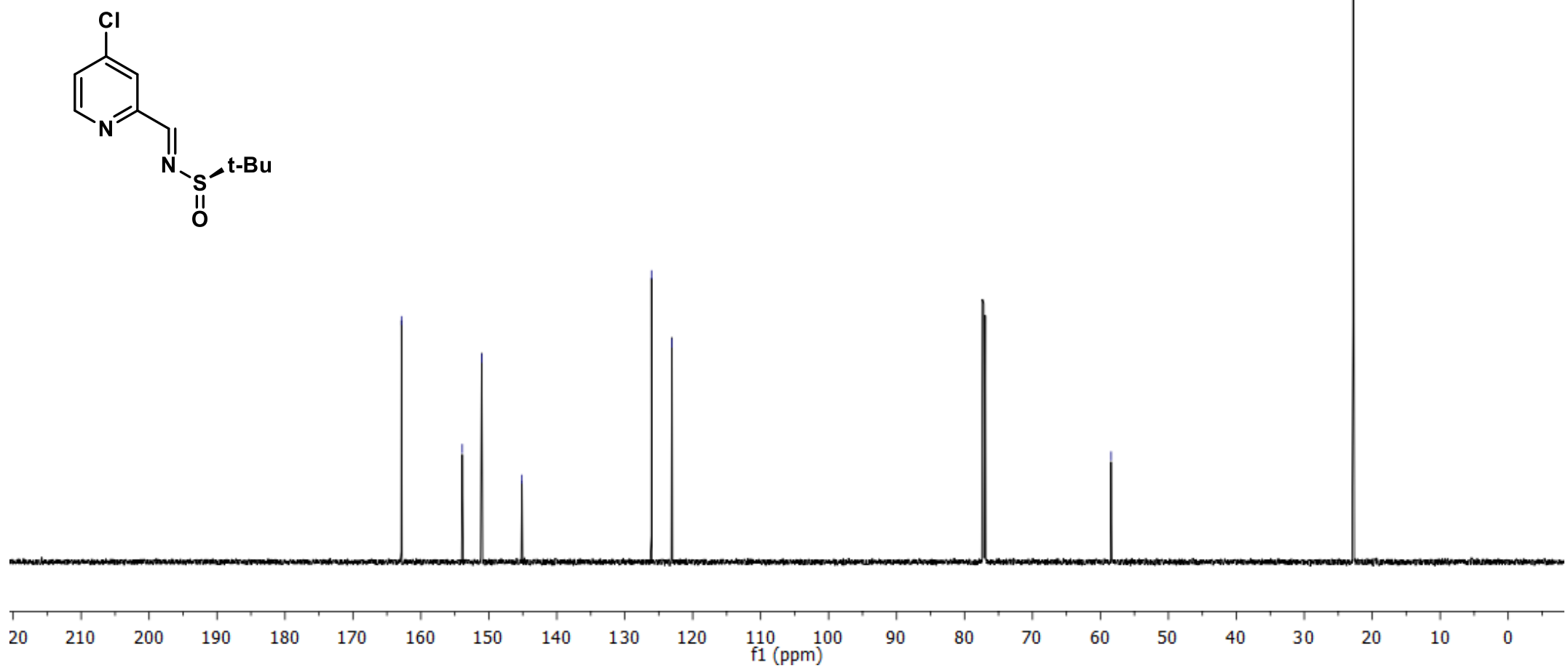


\section{Compound SI-10 ${ }^{1} \mathrm{H}$ NMR}

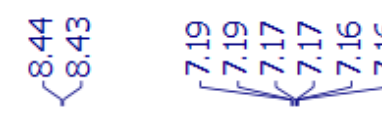

ำ

कू बิ

$\stackrel{\infty}{\stackrel{\circ}{\circ}}$

(1)

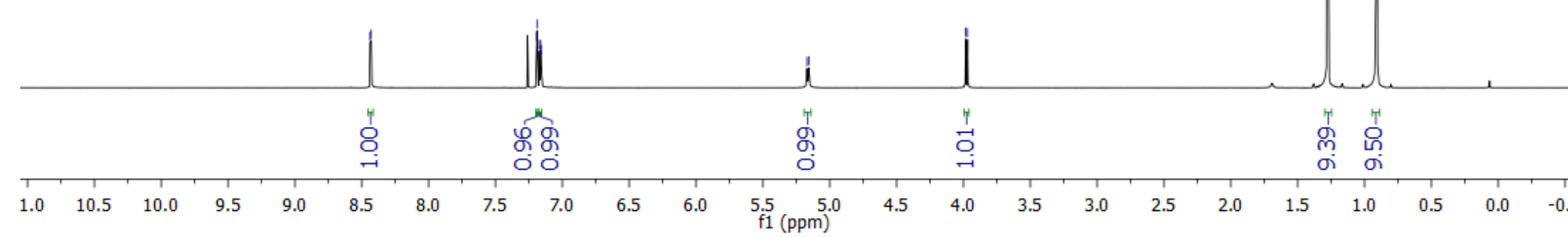

SI-103 


\section{Compound SI-10 ${ }^{13} \mathrm{C}$ NMR}

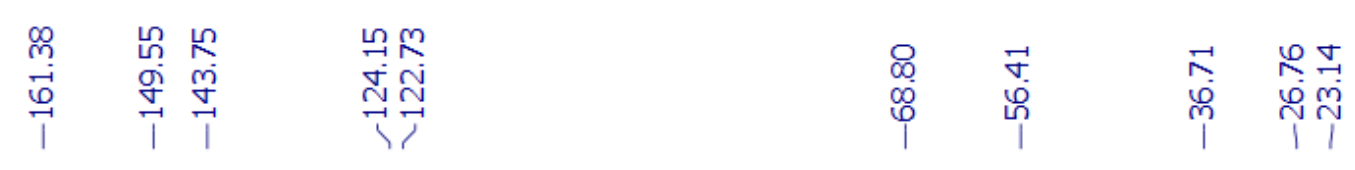

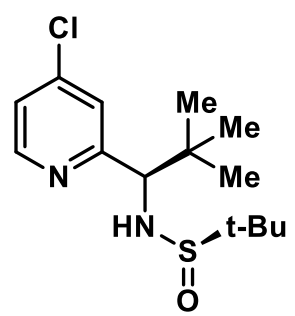

$\begin{array}{lllllllllll}210 & 200 & 190 & 180 & 170 & 160 & 150 & 140 & 130 & 120 & 110 \\ \mathrm{f} 1(\mathrm{ppm})\end{array}$ 


\section{Compound $9{ }^{1} \mathrm{H}$ NMR}

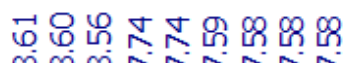

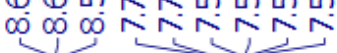

กำ



১্
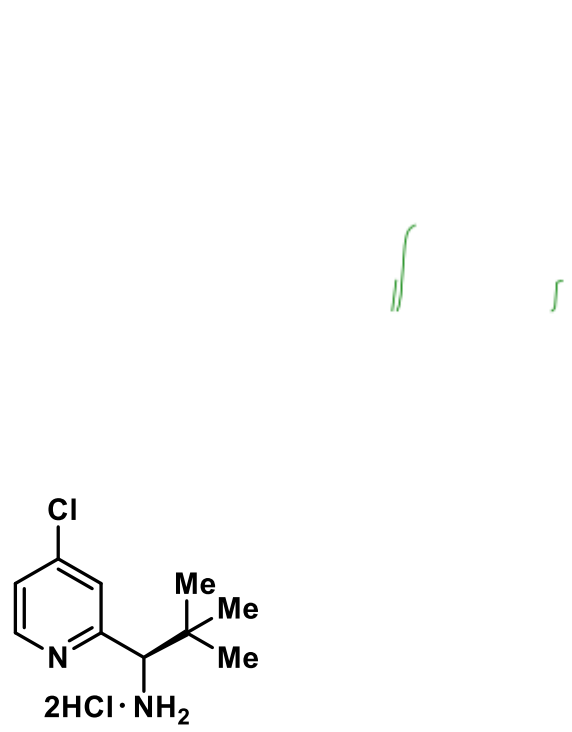

I 1

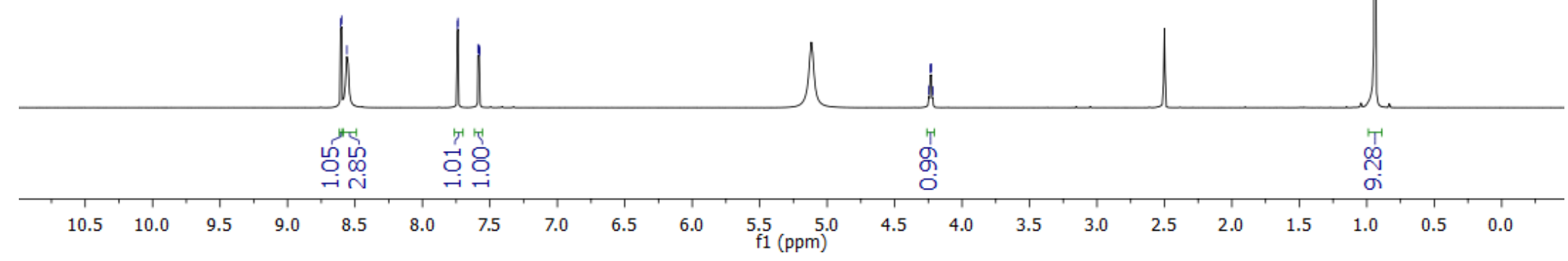

SI-105 


\section{Compoun $9{ }^{13} \mathrm{C}$ NMR}

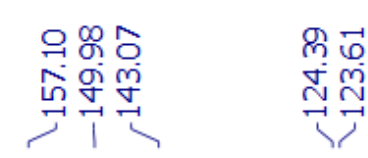

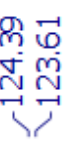

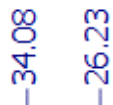
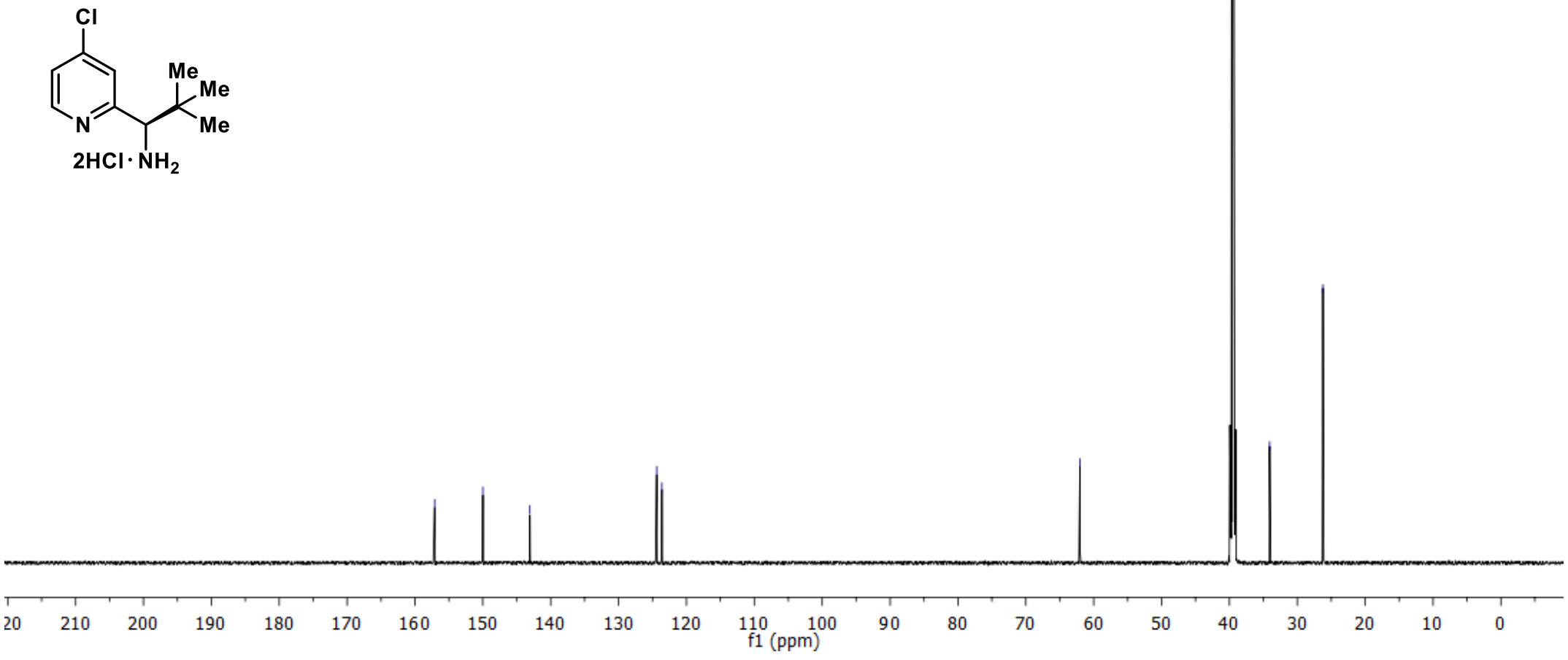

SI-106 


\section{Compound 10d ${ }^{1}$ H NMR}

$\underbrace{\infty \infty}_{\infty}$
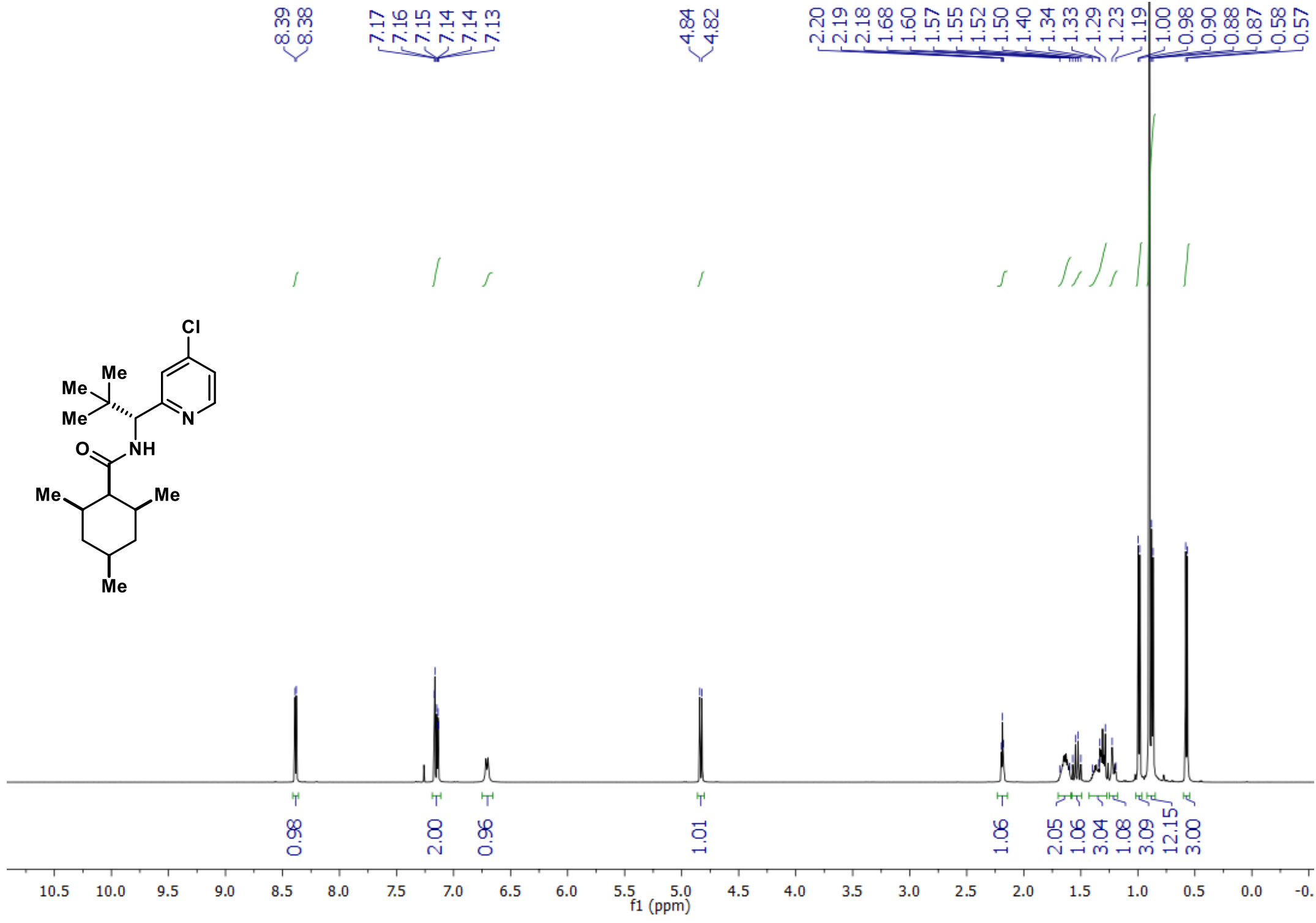


\section{Compound 10d ${ }^{13} \mathrm{C}$ NMR}

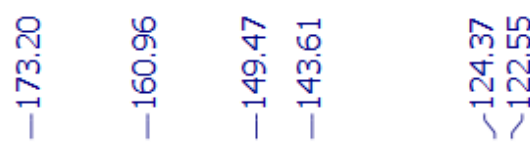

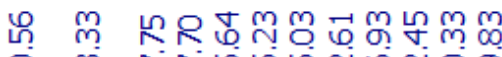

○ m
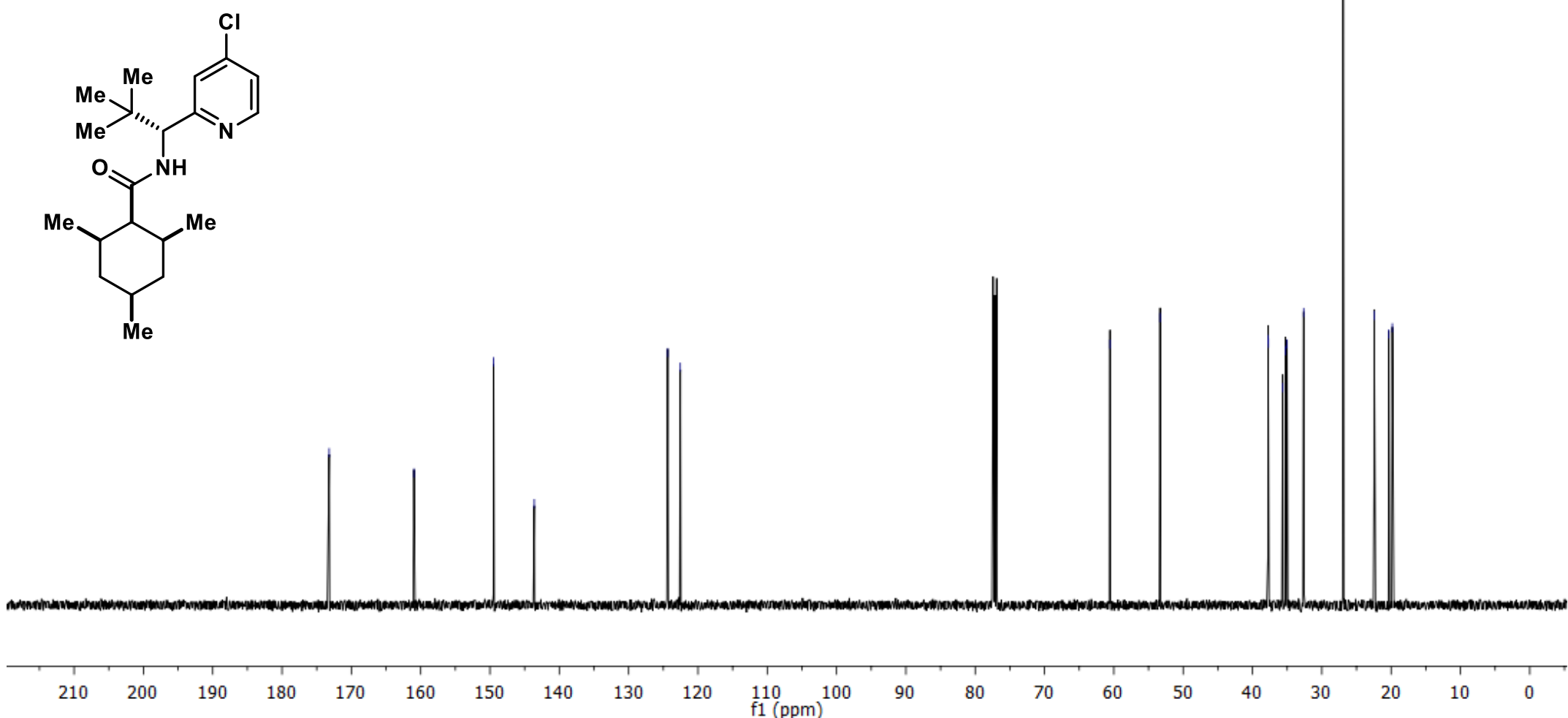


\section{Compound $11{ }^{1} \mathrm{H}$ NMR}

mom

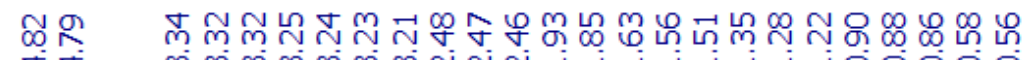

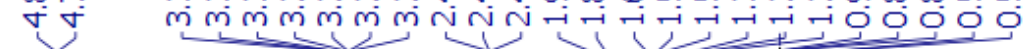<smiles>COCC1CC([N+]#N)CC(C)C1C(=O)NC(c1cc(Cl)ccn1)C(C)(C)C</smiles>

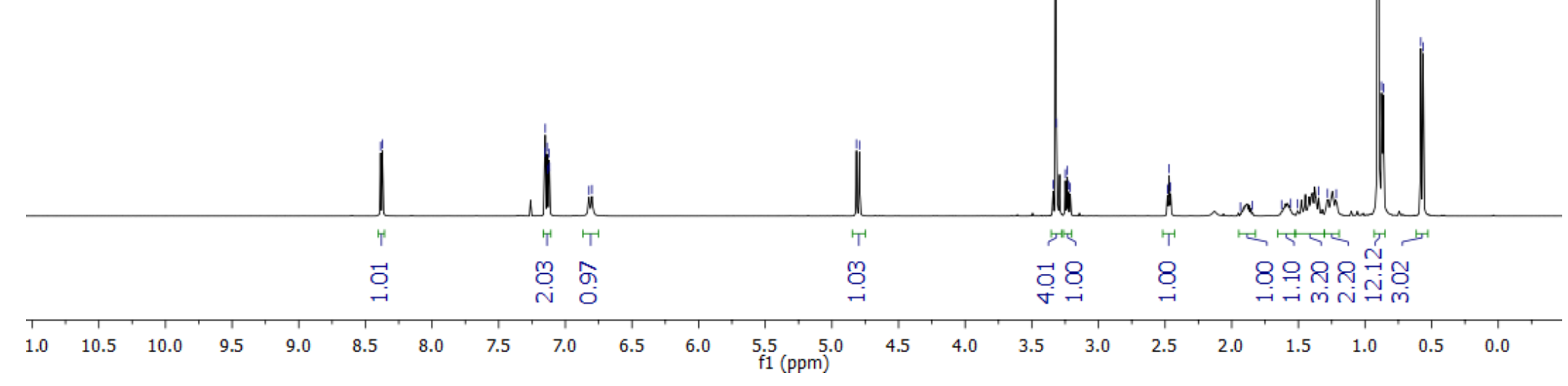




\section{Compound $11{ }^{13} \mathrm{C}$ NMR}

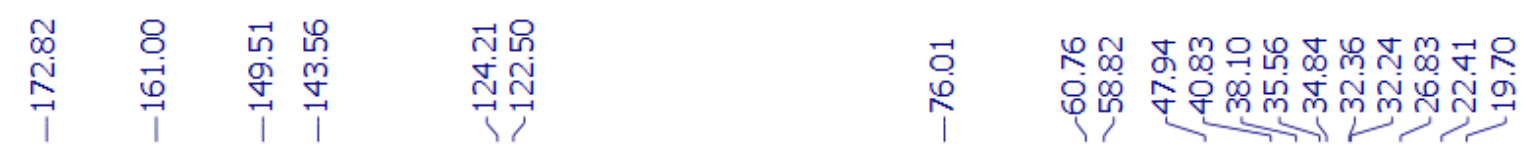<smiles>[M]C1CC(COC)C(C(=O)N[C@H](c2cc(Cl)ccn2)C(C)(C)C)[C@@H](C)C1</smiles>

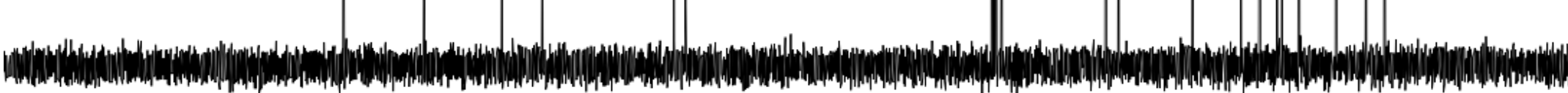

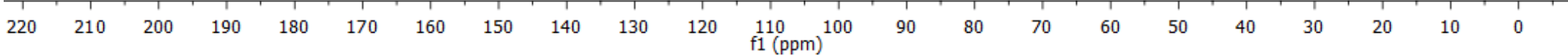




\section{Compound $12{ }^{1} \mathrm{H}$ NMR}

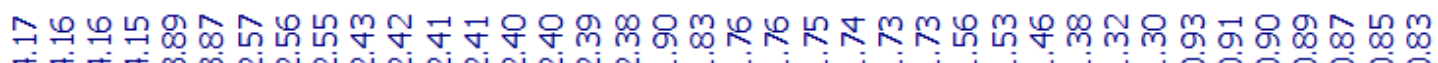

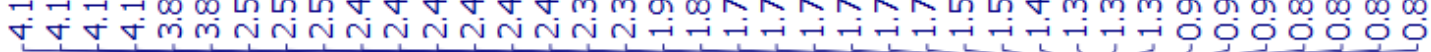
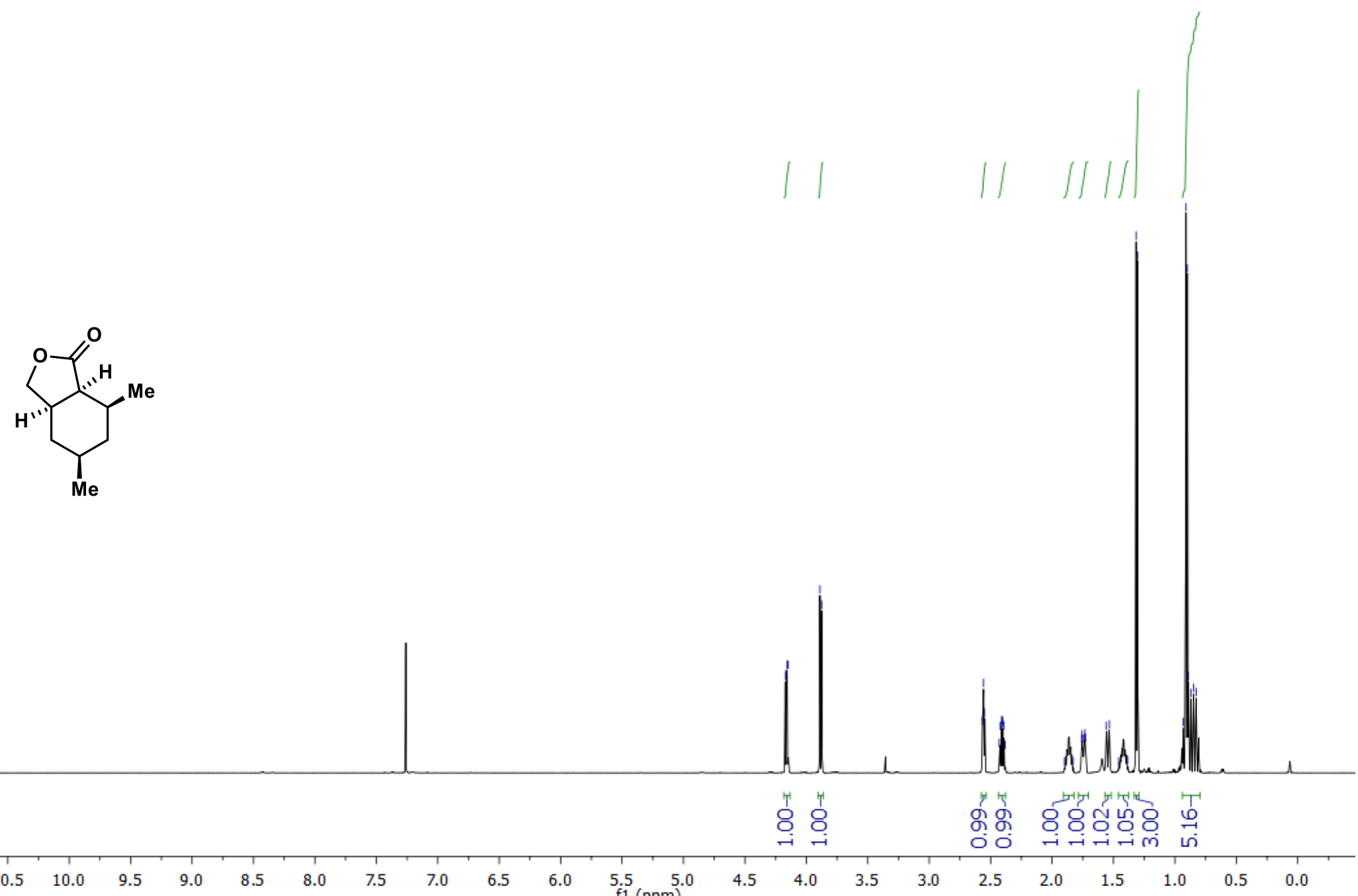

$1.0 \quad 10.5$

10.0

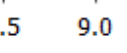

8.5

1
7.5

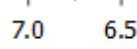

$\begin{array}{lll}6.0 & 5.5 & 5.0 \\ & & f 1(\mathrm{ppm})\end{array}$

SI-111 


\section{Compound $12{ }^{13} \mathrm{C}$ NMR}
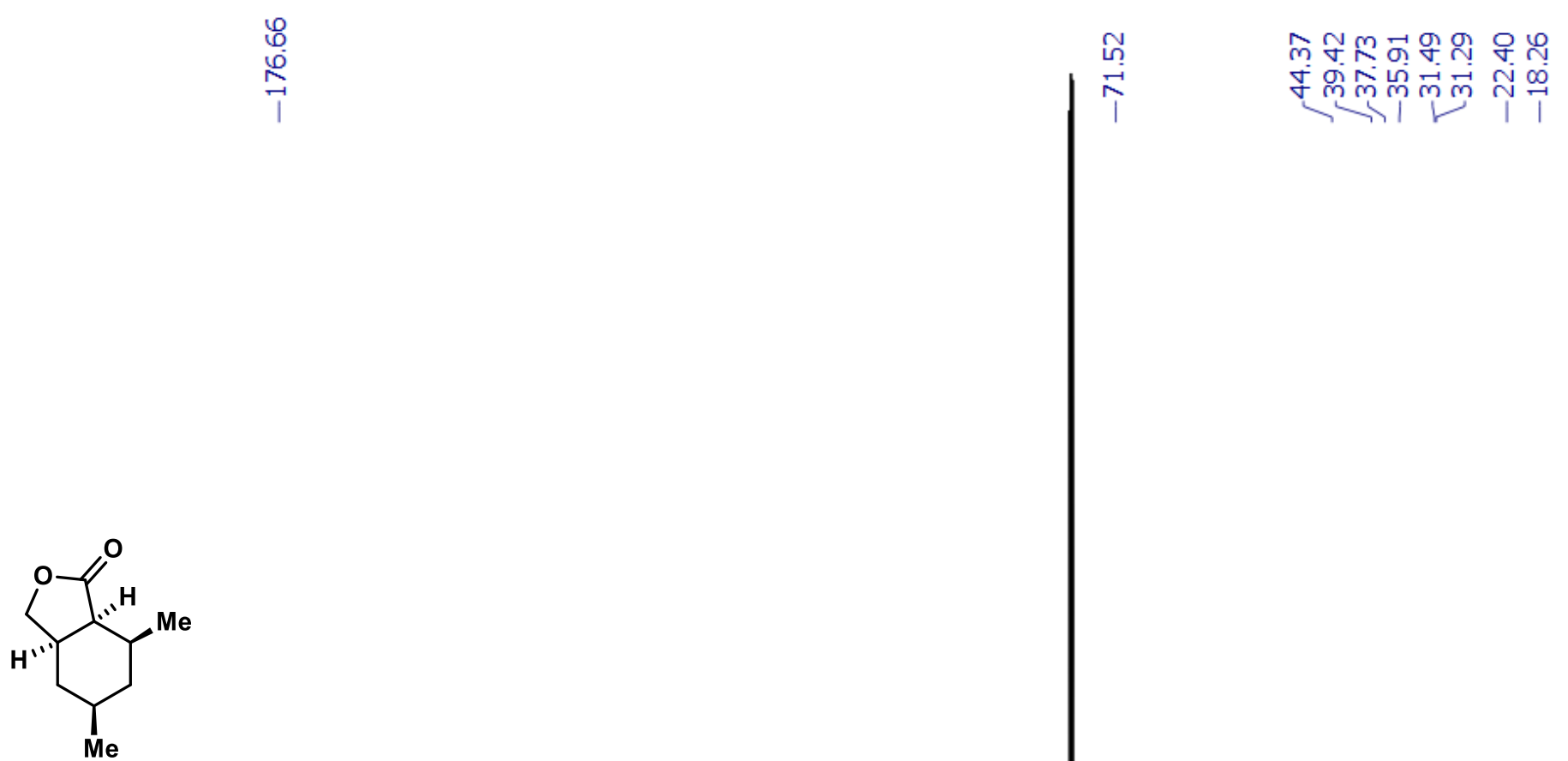

$\begin{array}{lllll}10 & 210 & 200 & 190 & 18\end{array}$

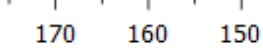

$\begin{array}{llll}140 & 130 & 120 & \begin{array}{c}110 \\ \mathrm{f} 1(\mathrm{ppm})\end{array}\end{array}$

90

80

70

$60 \quad 50$

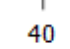

30

$20 \quad 10$

SI-112 


\section{Compound $14{ }^{\mathbf{1}} \mathrm{H}$ NMR}

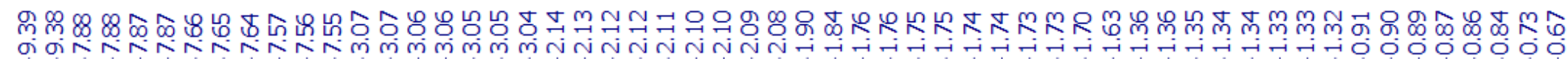<smiles>C[C@@H]1CC([N+](=O)[O-])C[C@H](C=O)[C@H]1CCc1ccccc1</smiles>

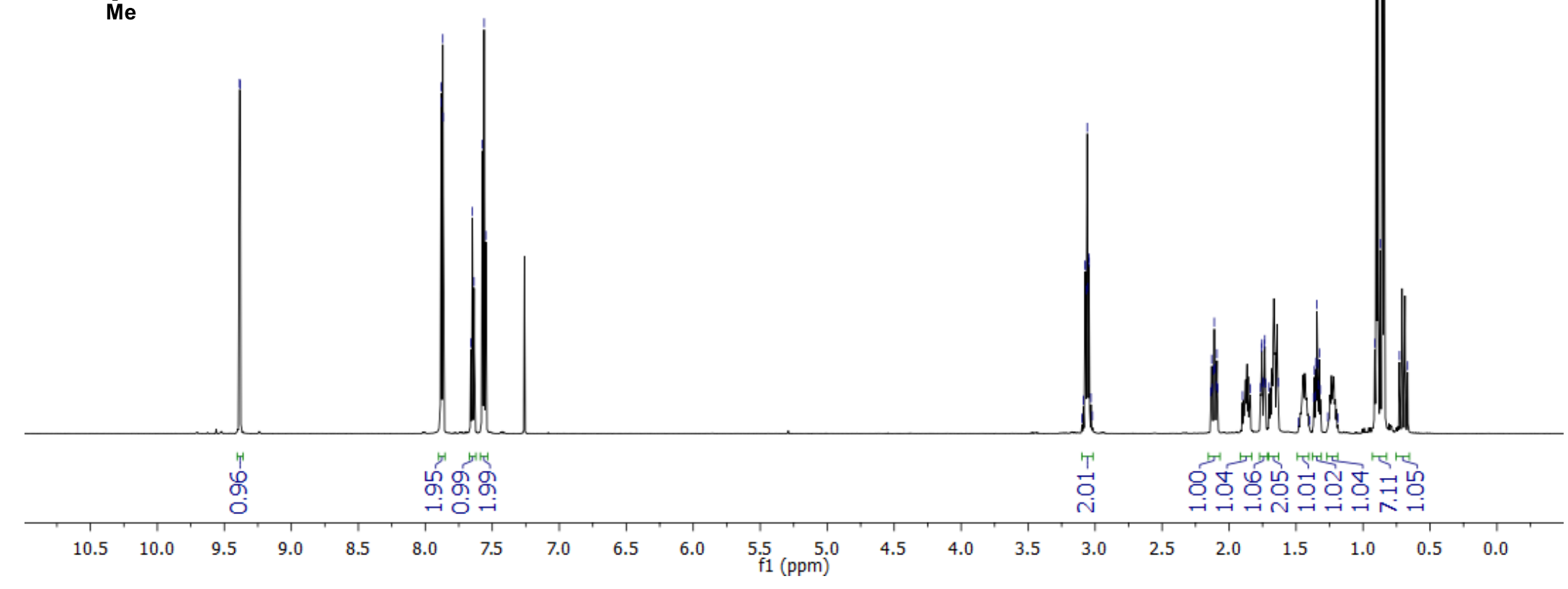




\section{Compound $14{ }^{13} \mathrm{C}$ NMR}

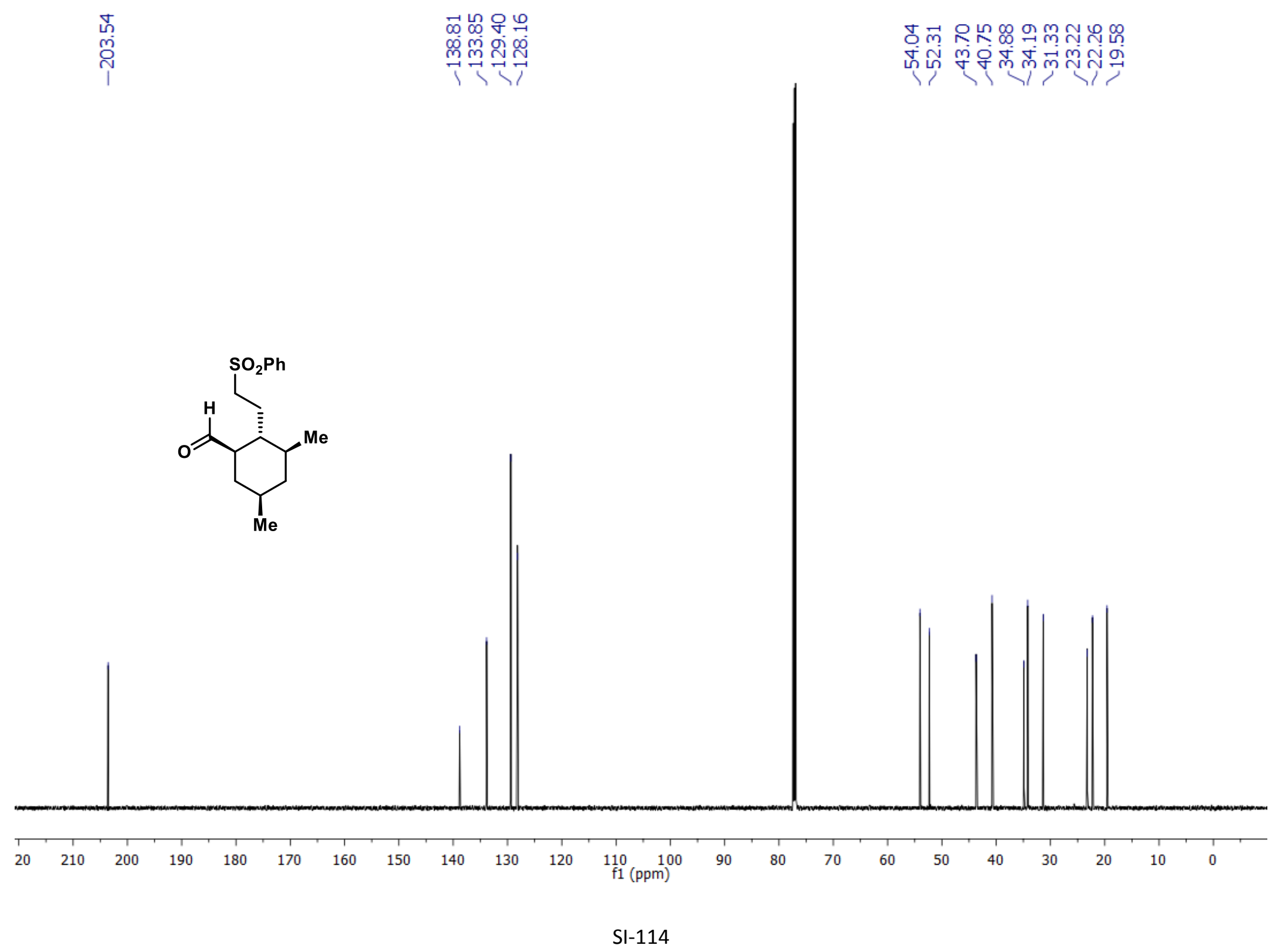




\section{Compound SI-12 ${ }^{1} \mathrm{H}$ NMR}

\&

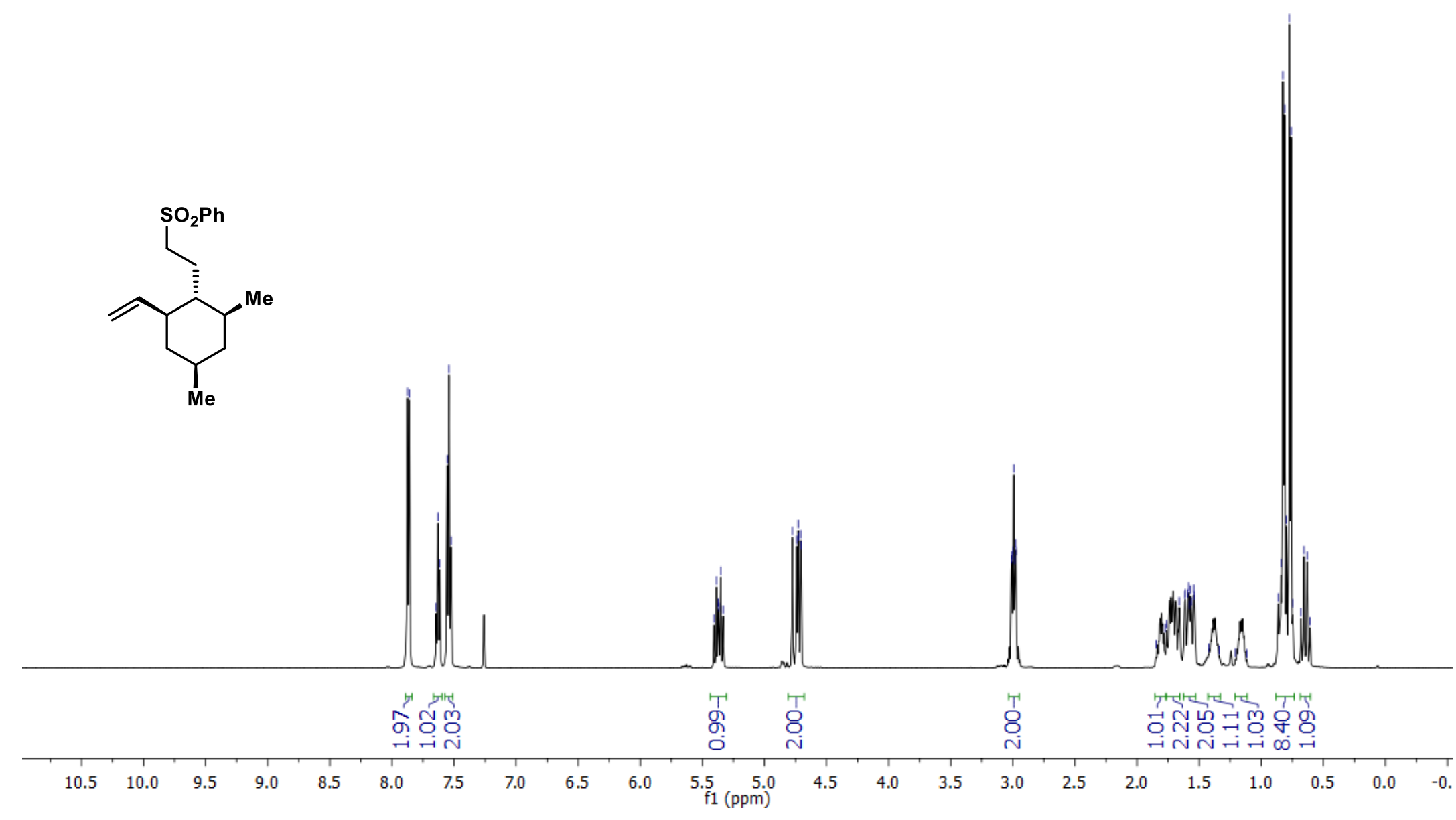
$\int 1$ 


\section{Compound SI-12 ${ }^{13} \mathrm{C}$ NMR}

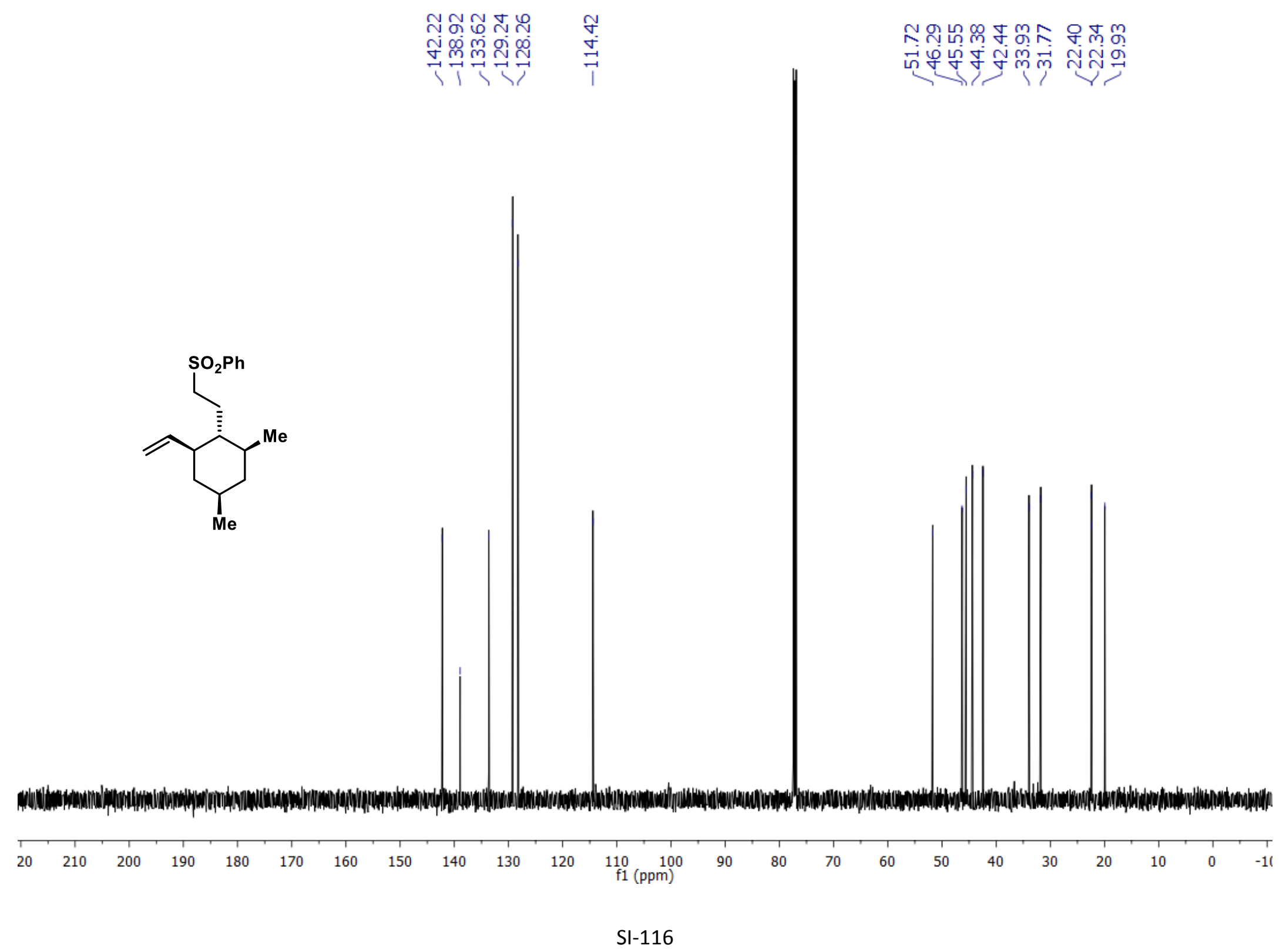




\section{Compound $15{ }^{1} \mathrm{H}$ NMR}

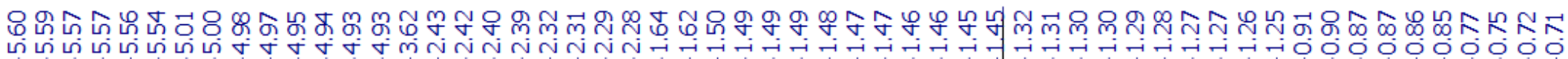
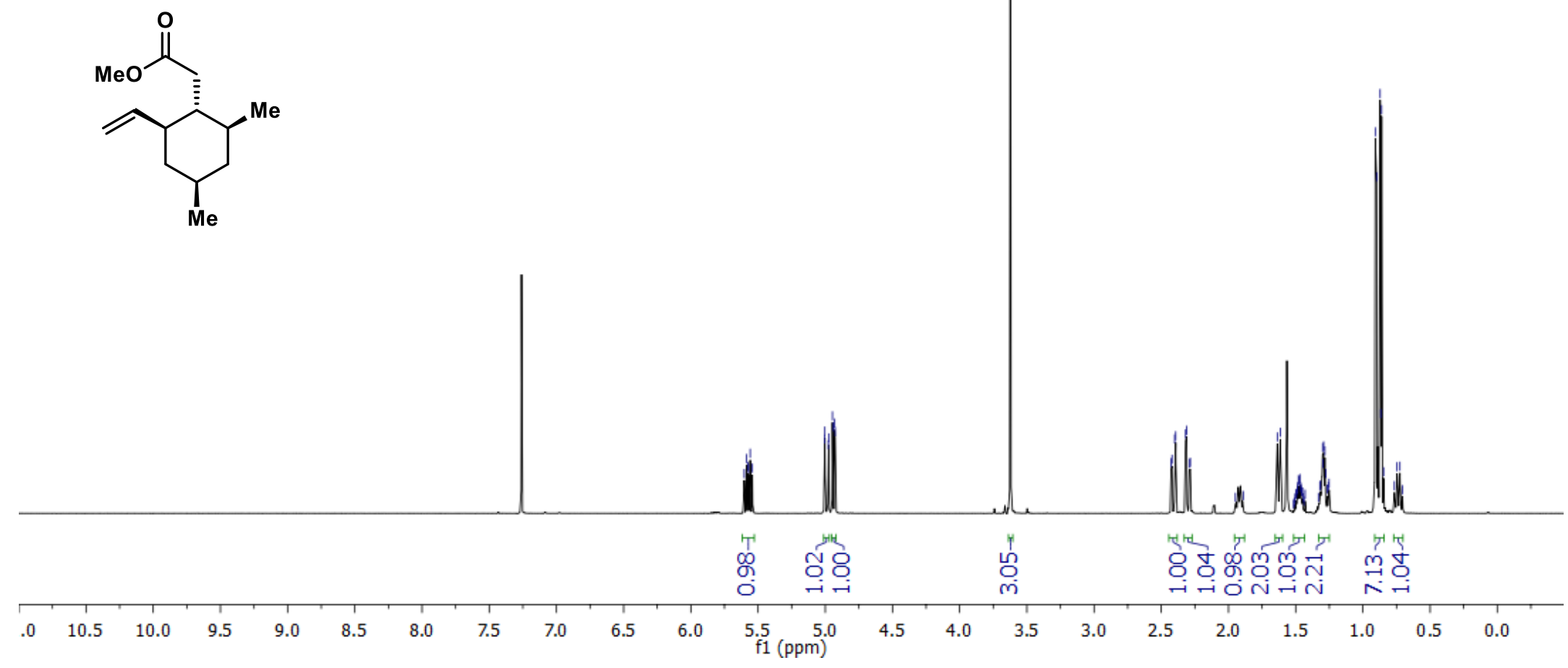


\section{Compound $15{ }^{13} \mathrm{C}$ NMR}
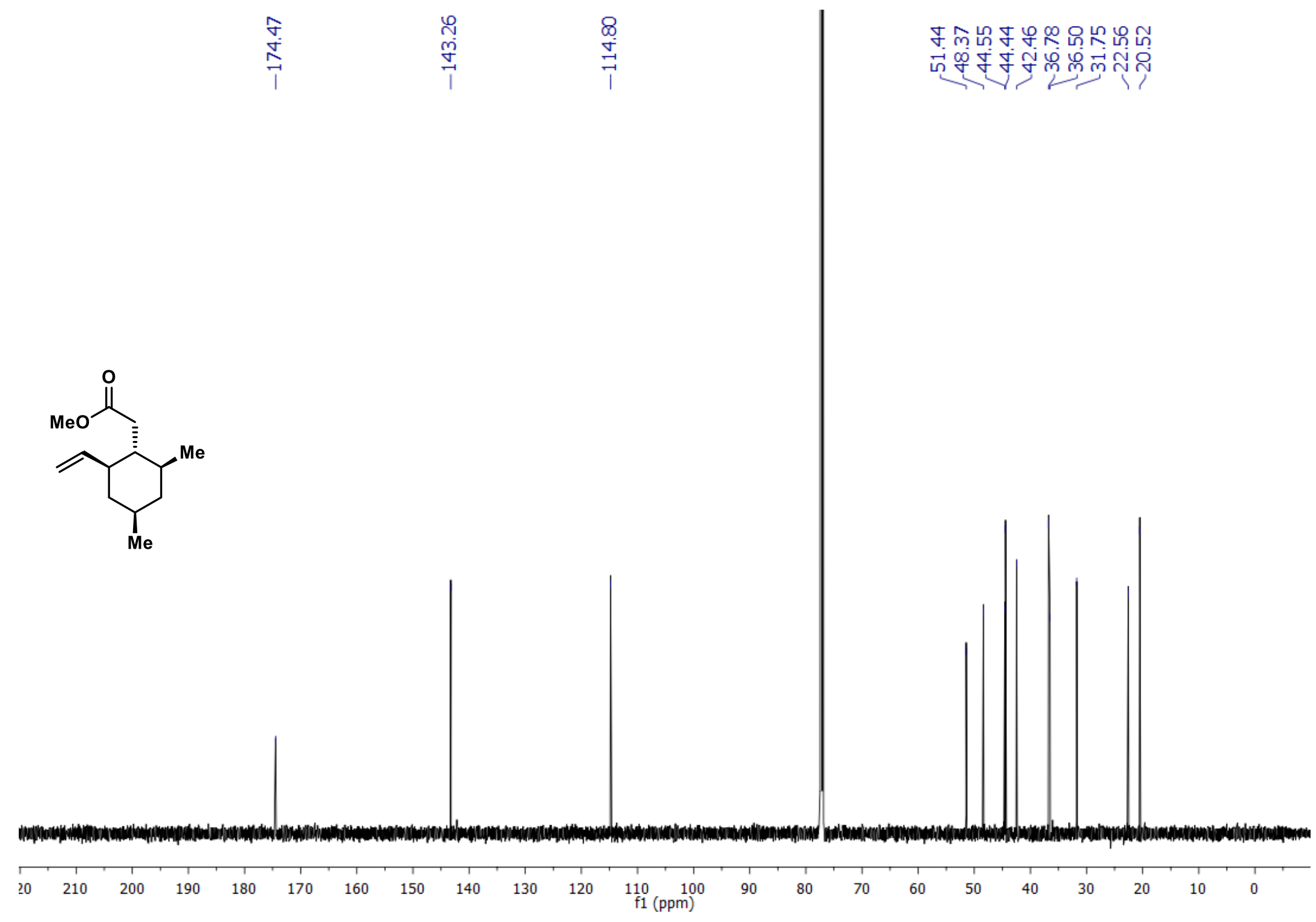


\section{Compound $6{ }^{1} \mathrm{H}$ NMR}

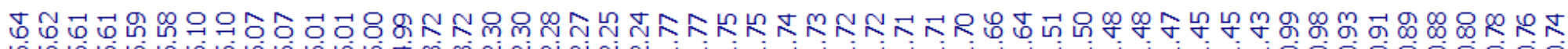

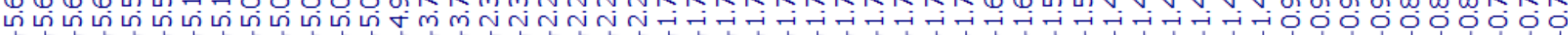<smiles>C=CC1CC([N+](=O)[O-])C[C@H](C)[C@H]1C(C(=O)O)C(=O)O</smiles>
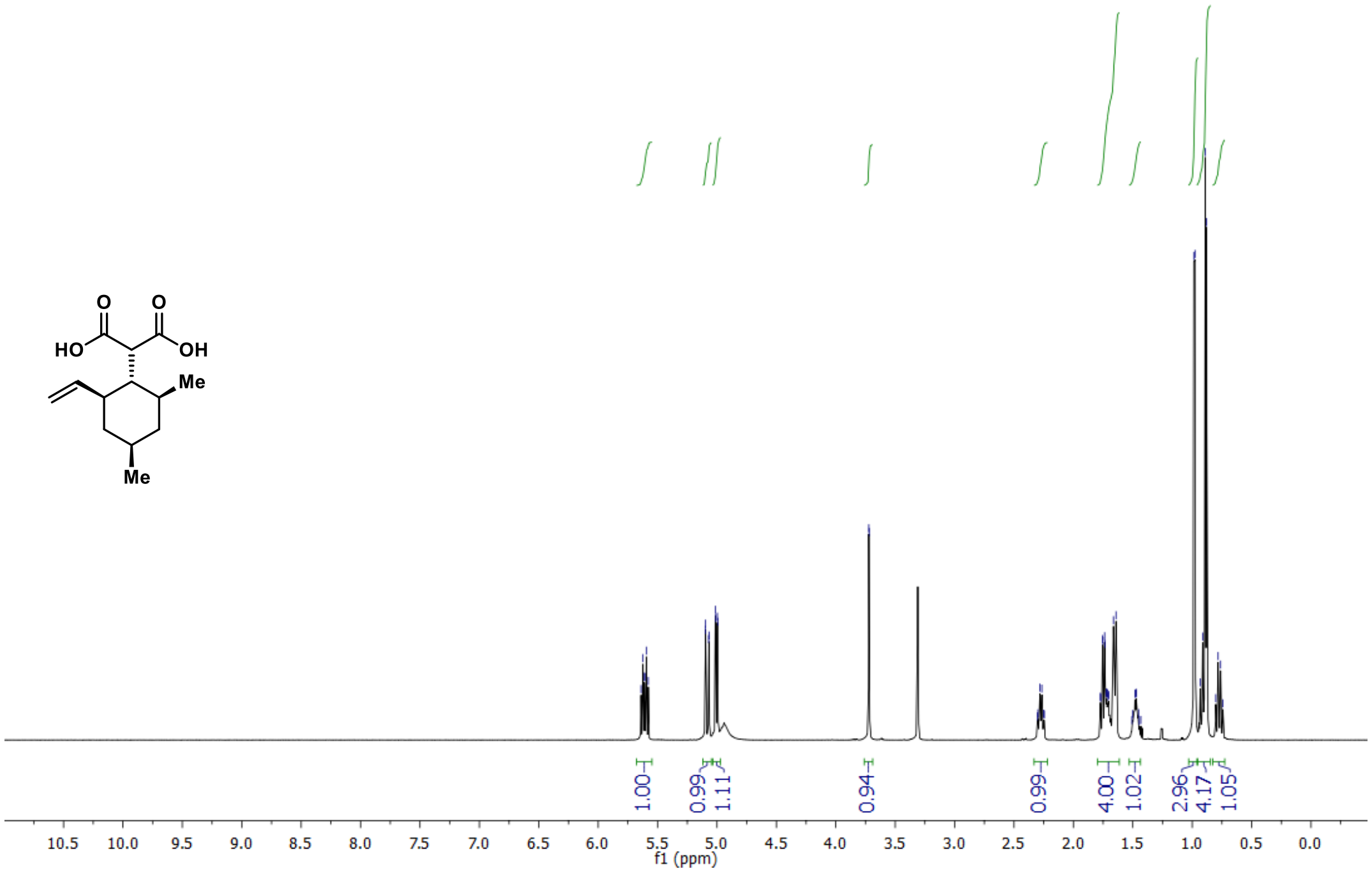


\section{Compound $6{ }^{13} \mathrm{C}$ NMR}

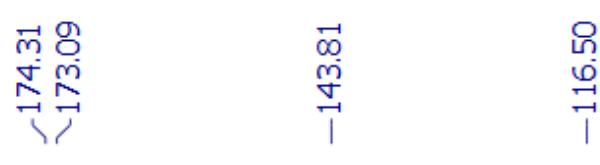

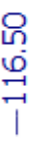

นํำ

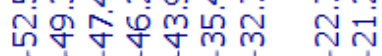<smiles>C=CC1CC([N+](=O)[O-])C[C@H](N)C1C(C(=O)O)C(=O)O</smiles>

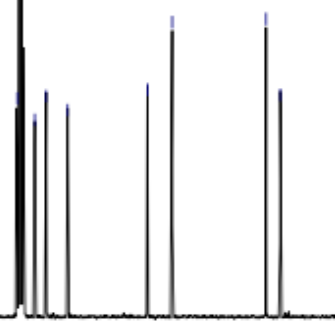

210 


\section{Compound 6 HSQC}

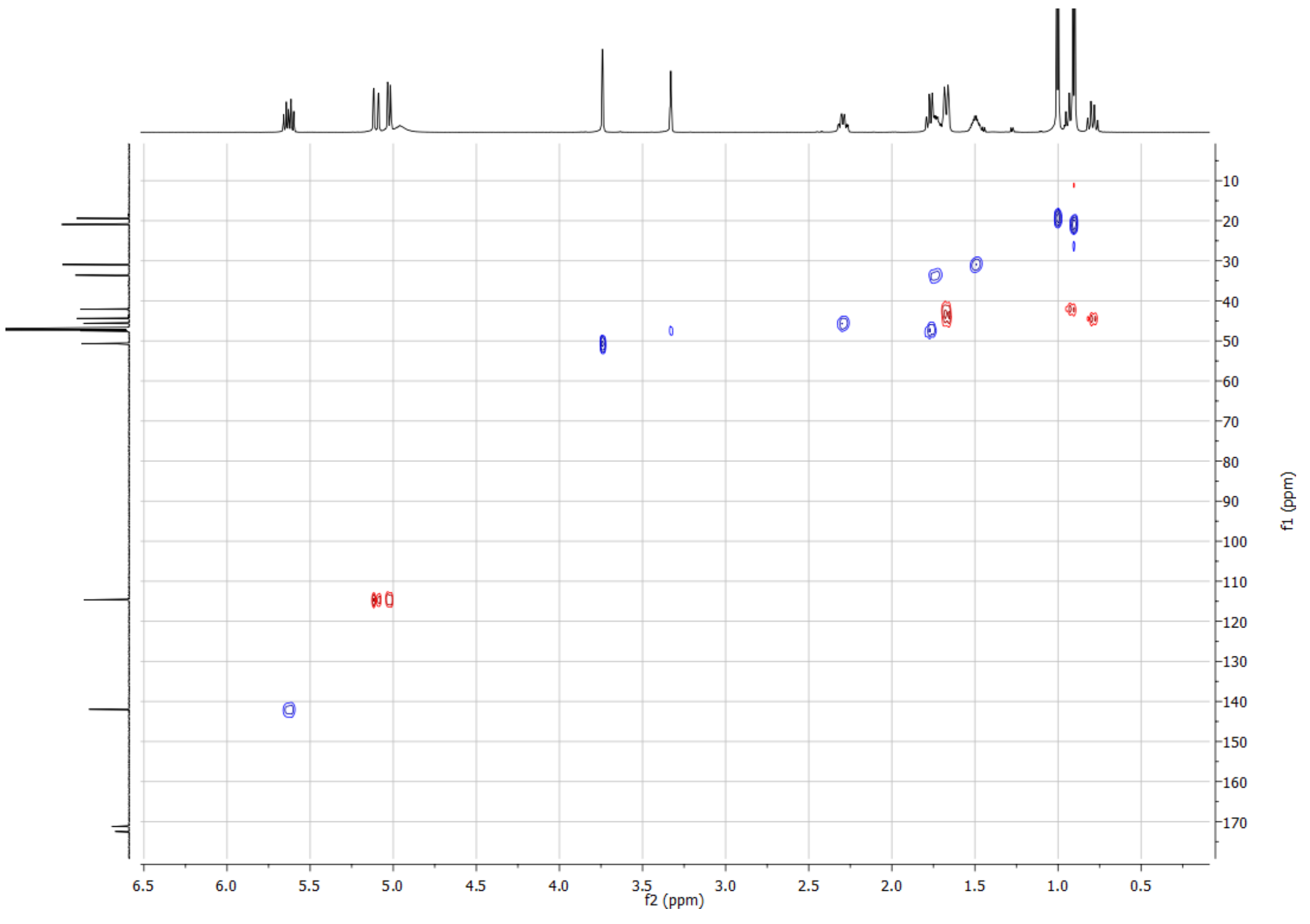




\section{Compound $17{ }^{1} \mathrm{H}$ NMR}

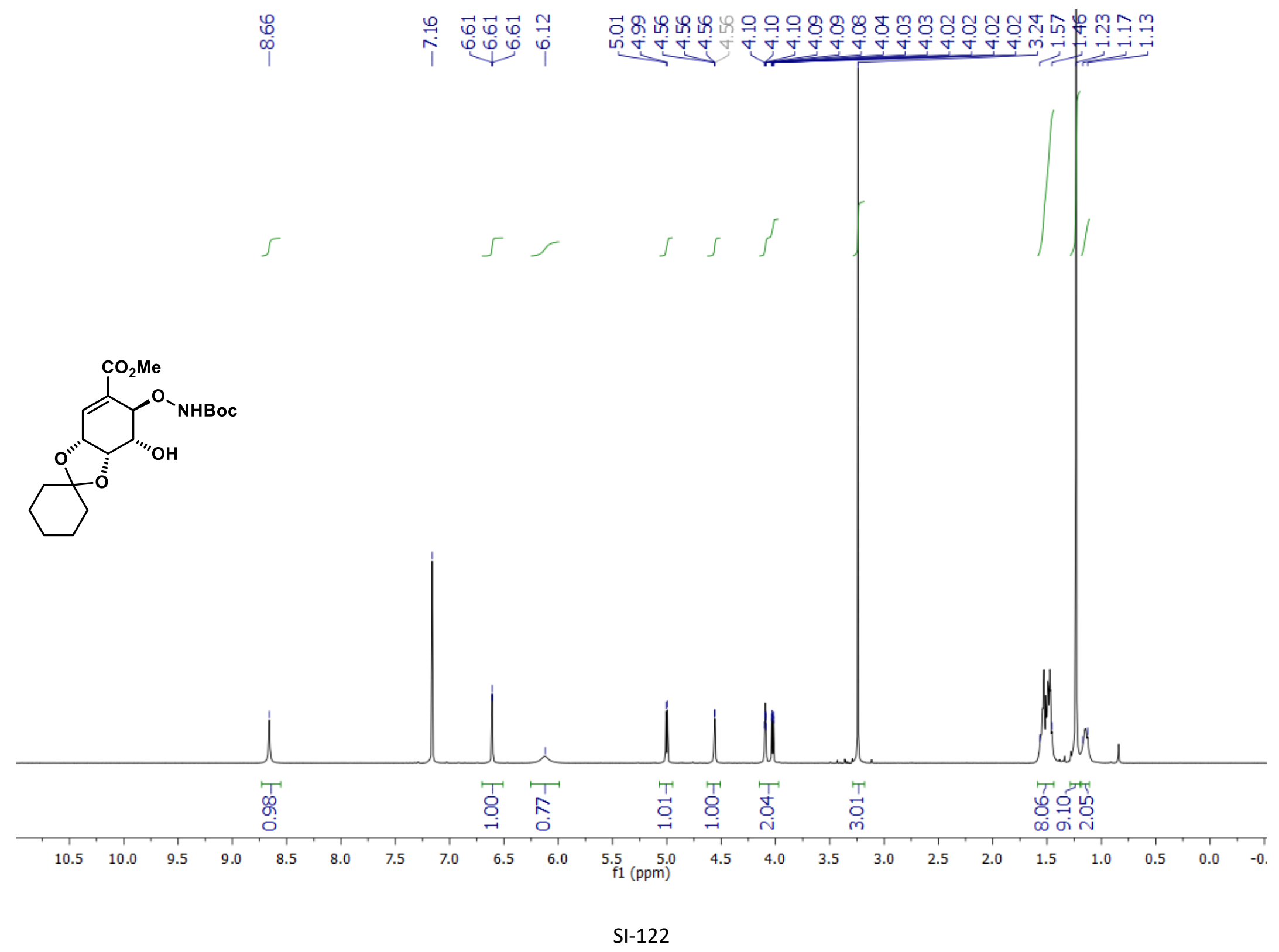




\section{Compound $17{ }^{13} \mathrm{C}$ NMR}

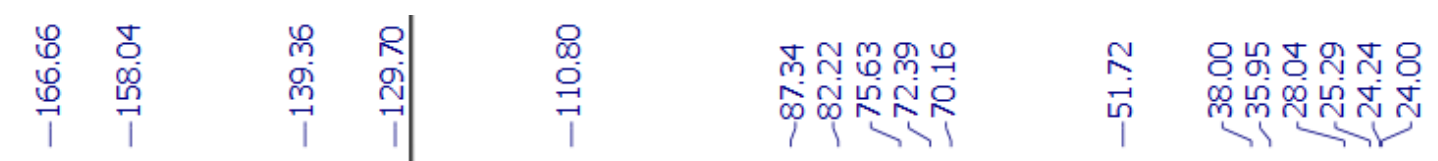
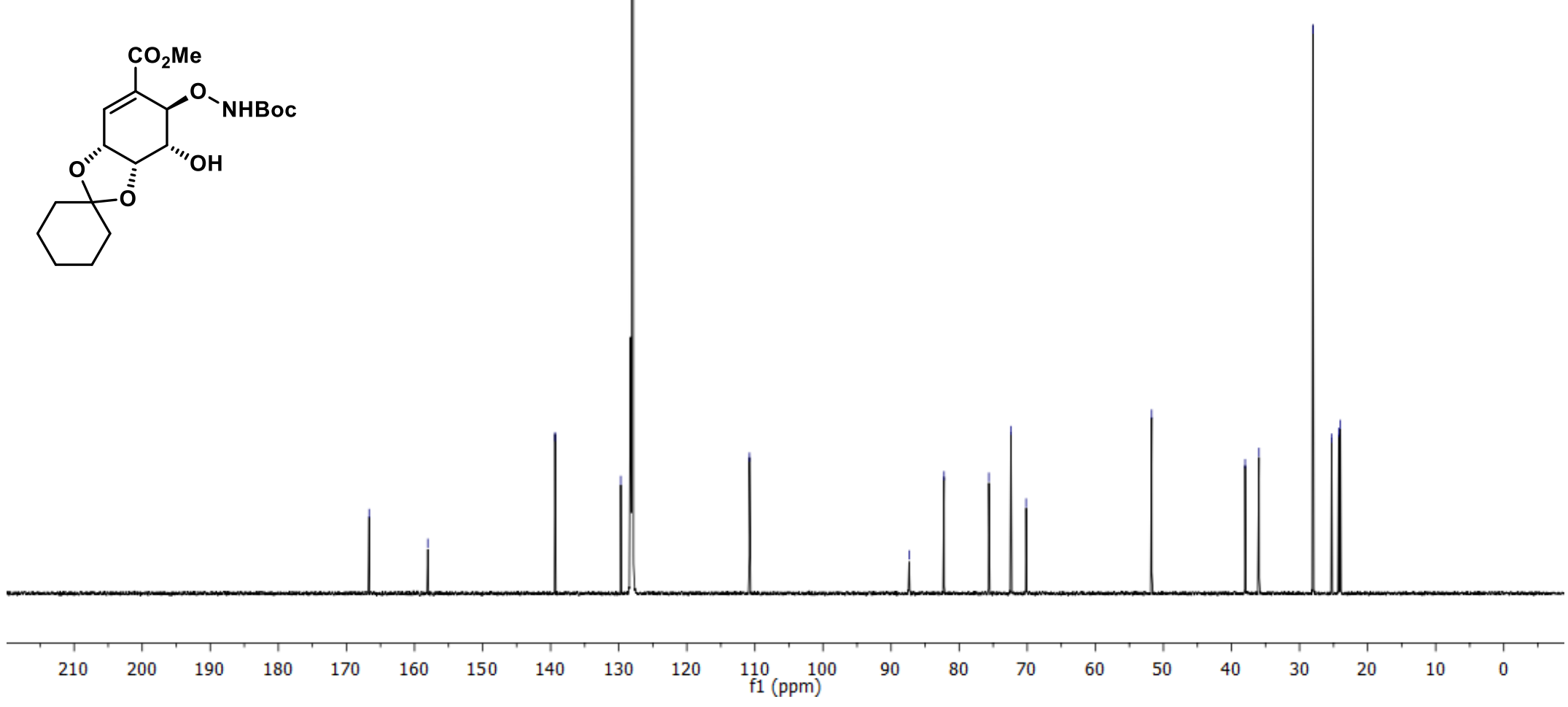


\section{Compound 17 COSY}

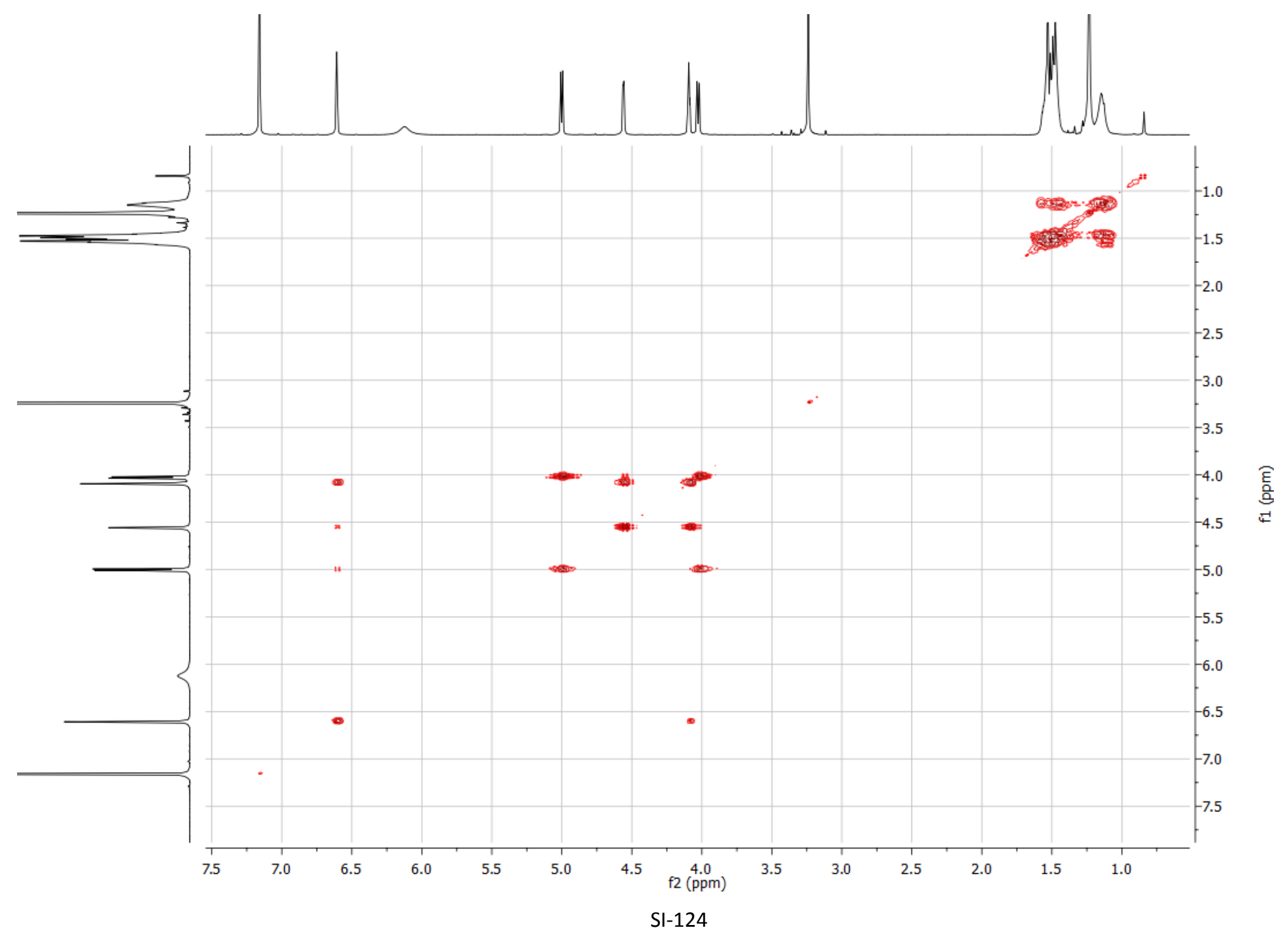




\section{Compound 17 HSQC}

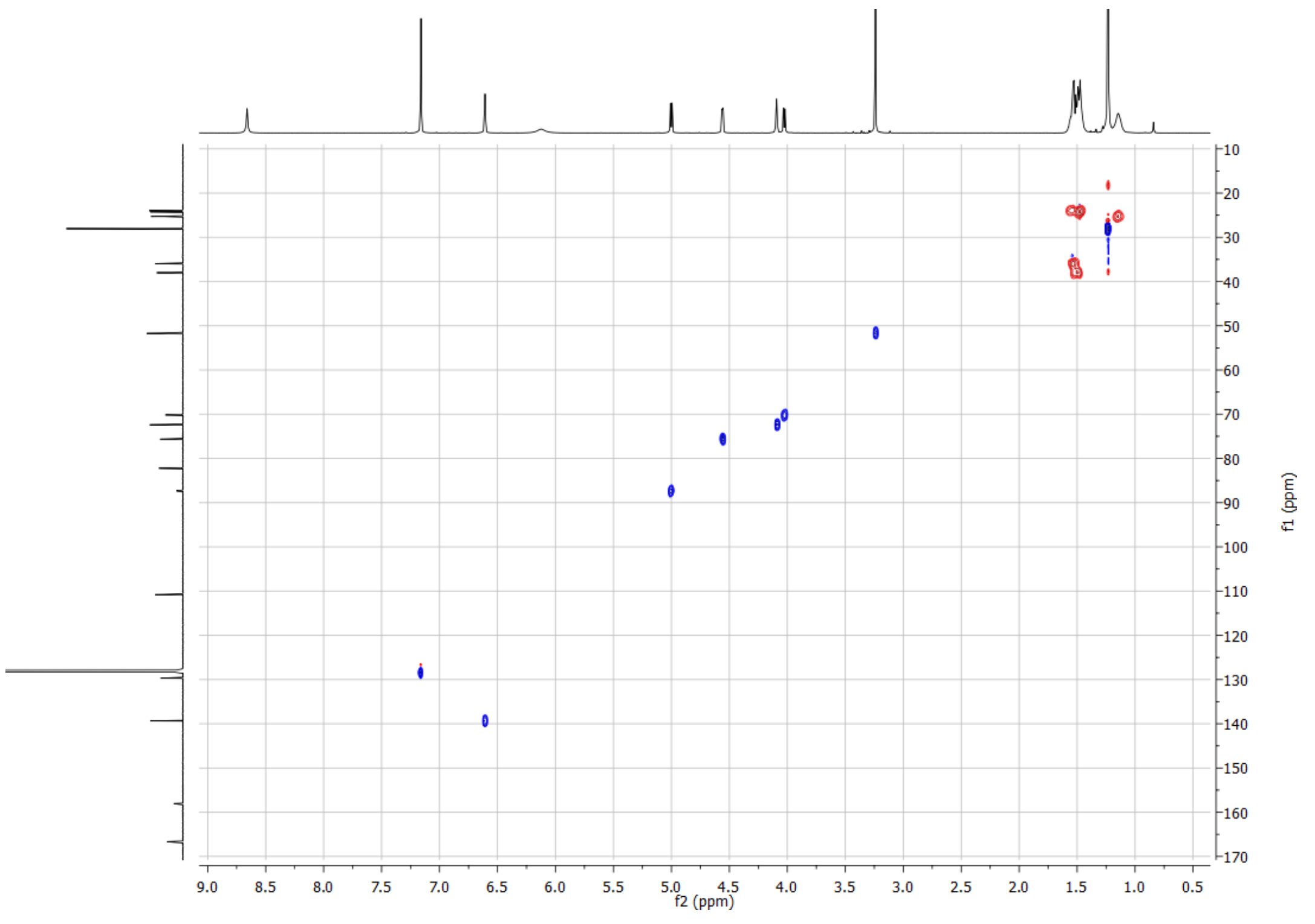




\section{Compound 17 NOESY}

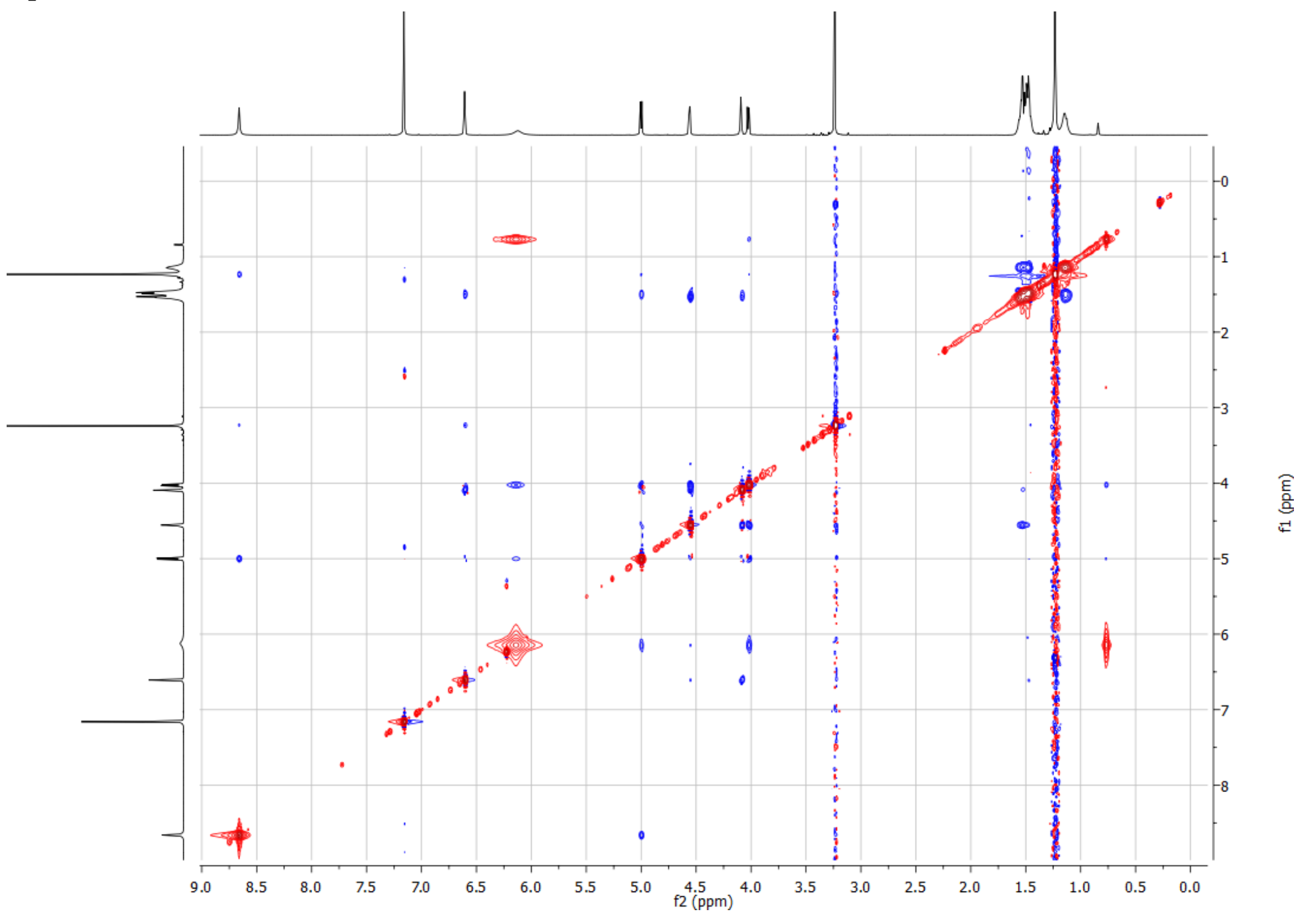




\section{Compound 17 HMBC}

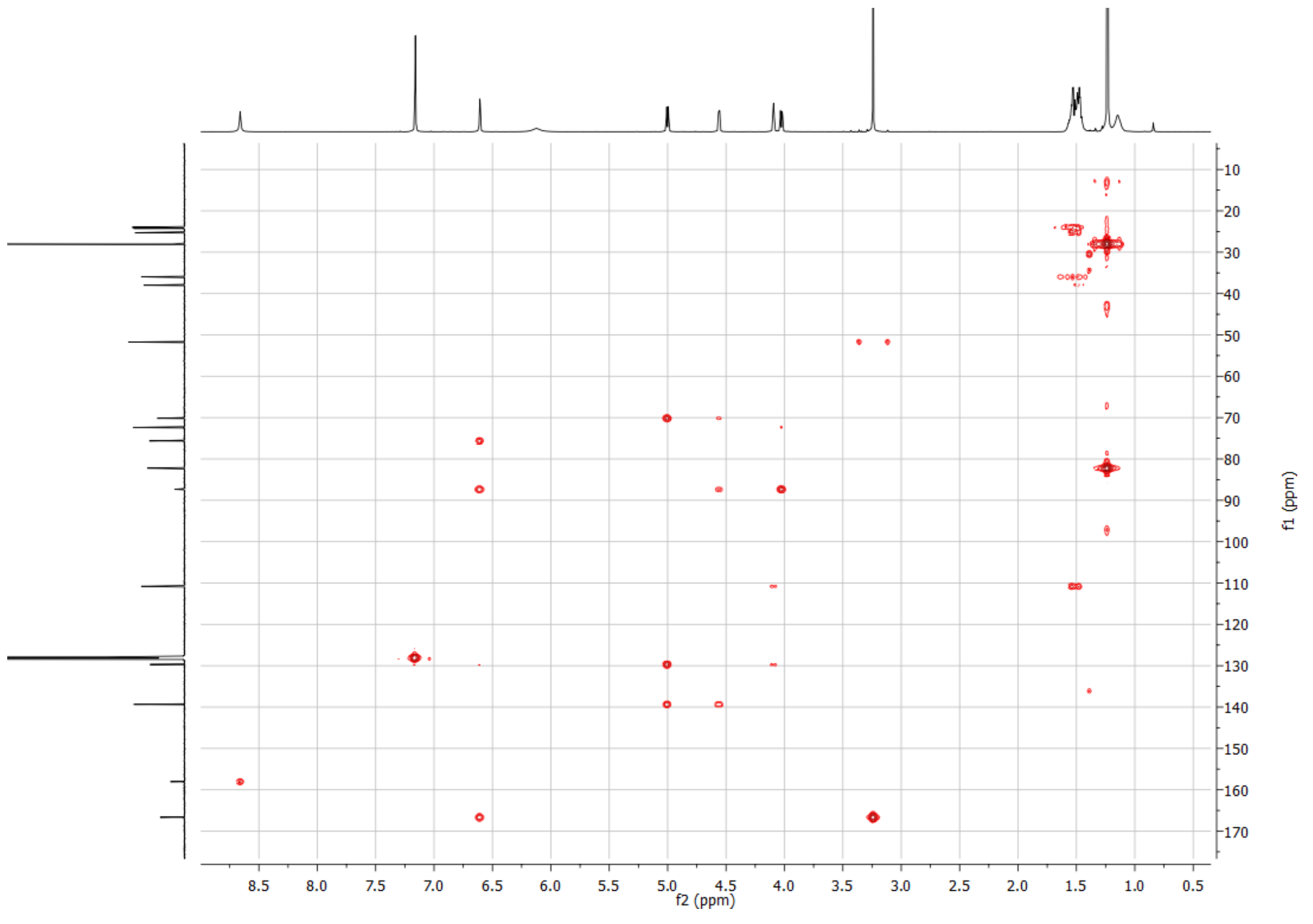




\section{Compound SI-13 ${ }^{1} \mathrm{H}$ NMR}

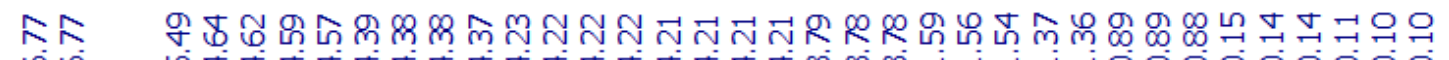

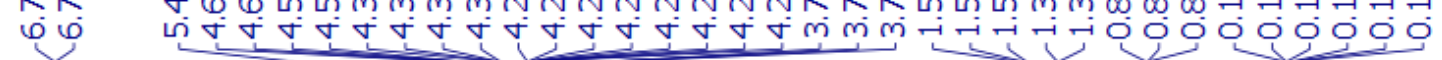
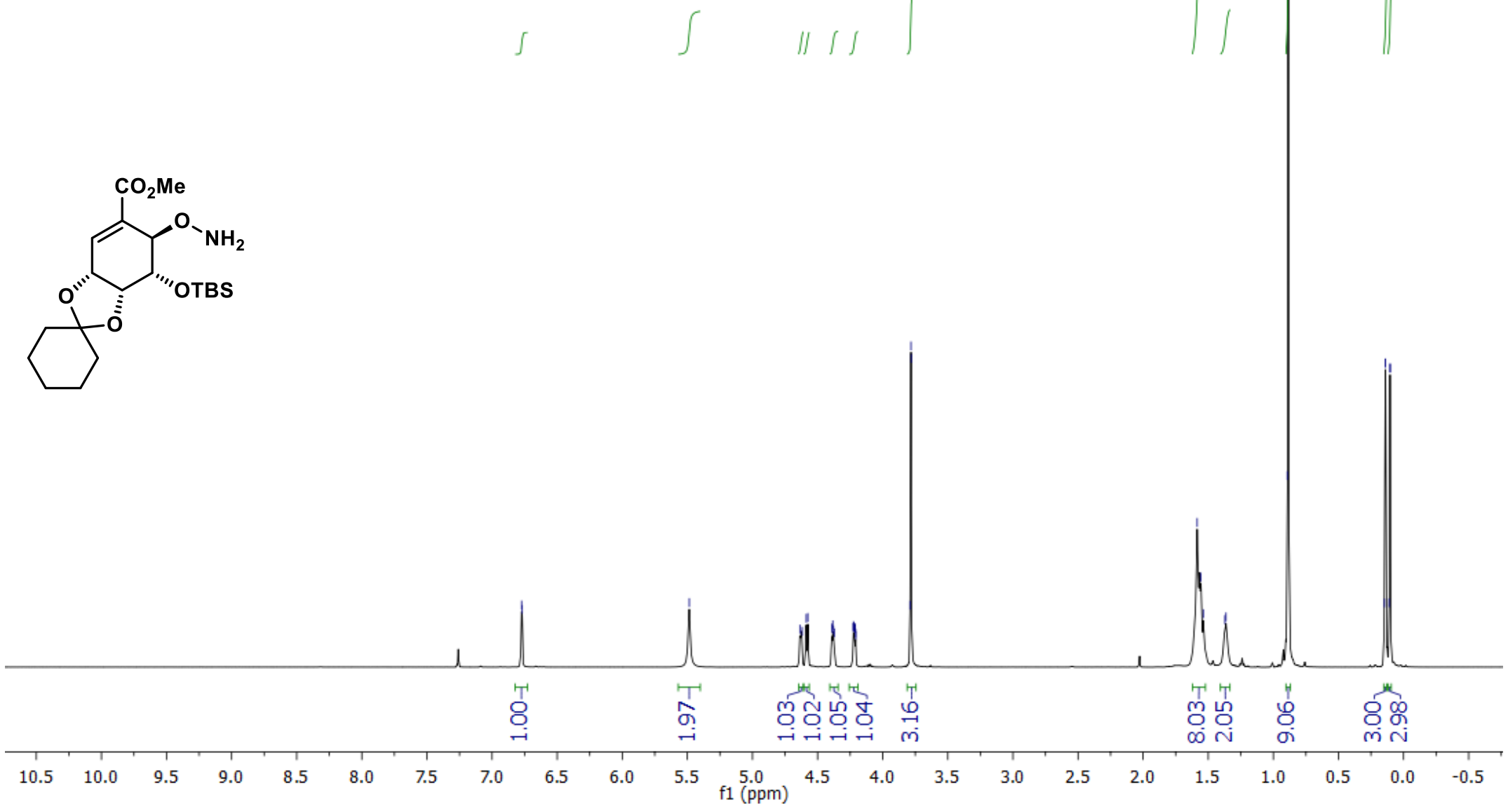


\section{Compound SI-13 ${ }^{13} \mathrm{C}$ NMR}

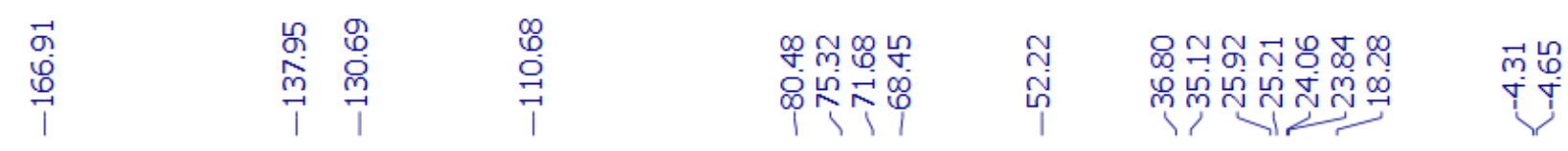

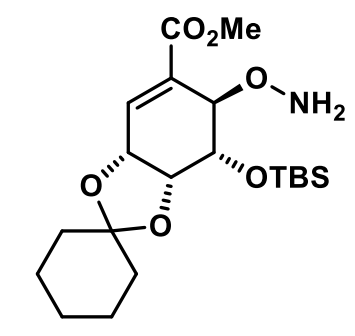

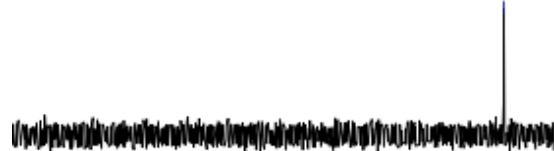

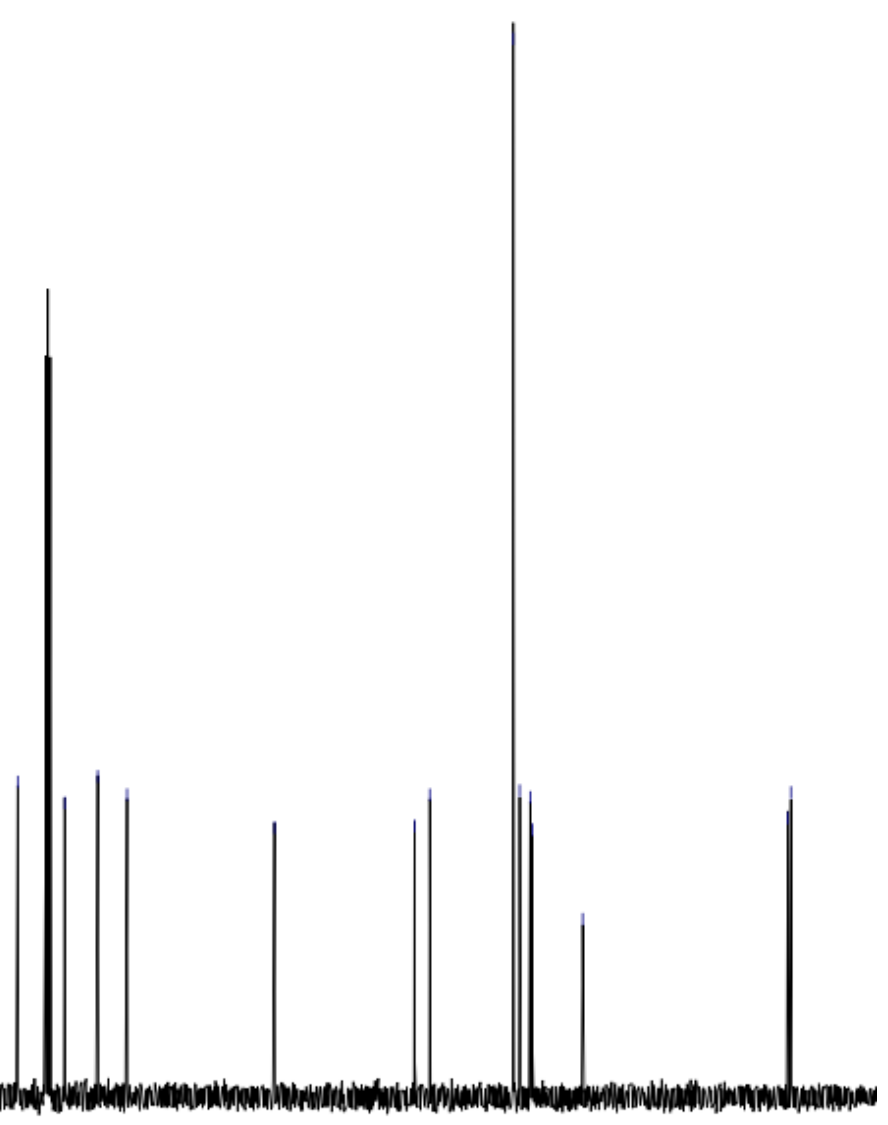

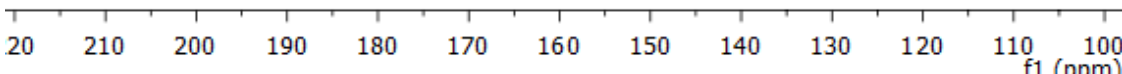

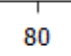

60

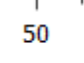

40

30

10

$-10$

SI-129 


\section{Compound $5{ }^{1} \mathrm{H}$ NMR}

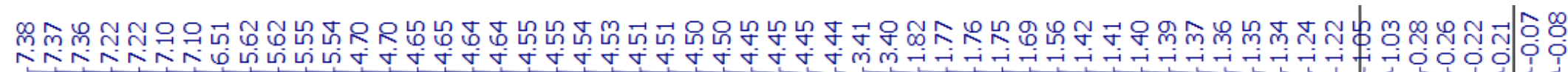

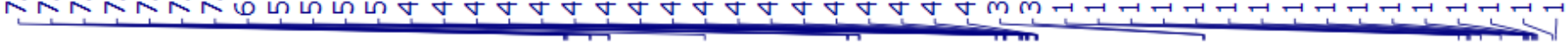
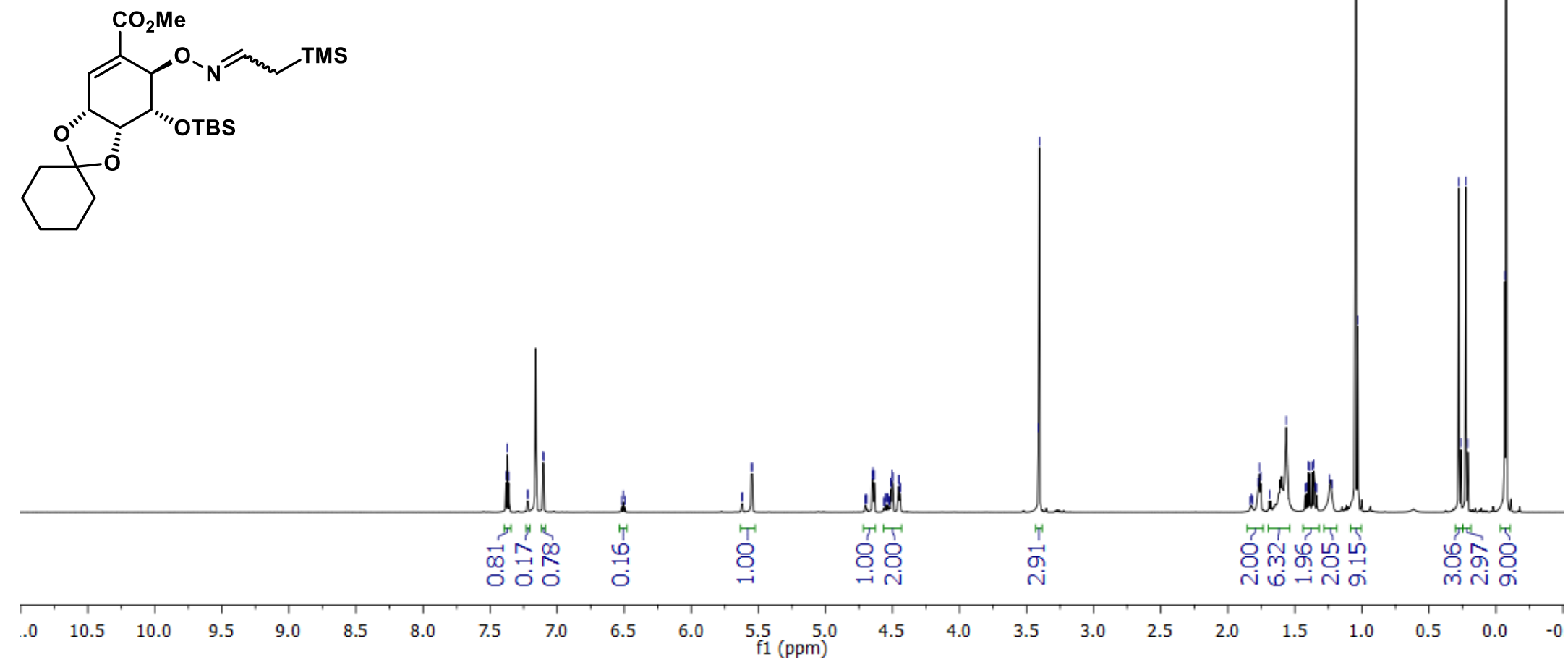


\section{Compound $5{ }^{13} \mathrm{C}$ NMR}

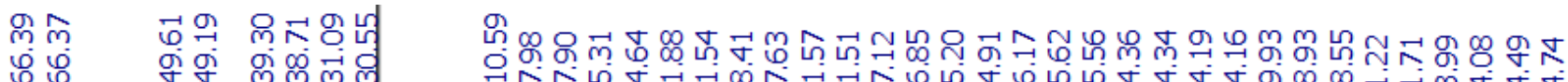

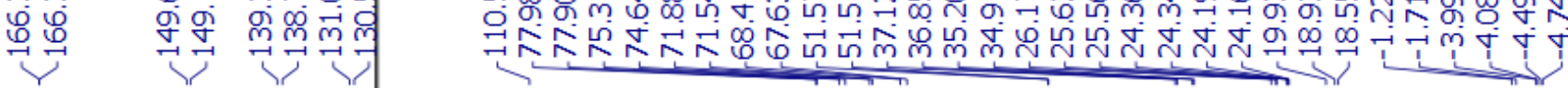

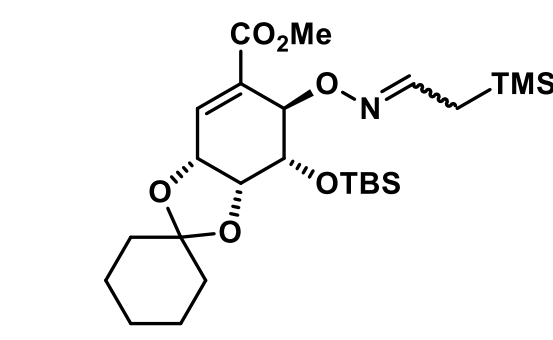

$210 \quad 200 \quad 190 \quad 180$
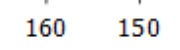

$110 \quad 100$

80

20 


\section{Compound des-TMS-5 ${ }^{1} \mathrm{H}$ NMR}

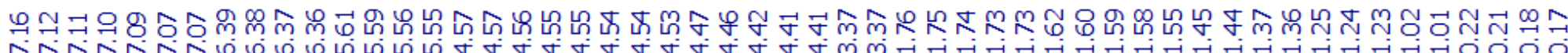

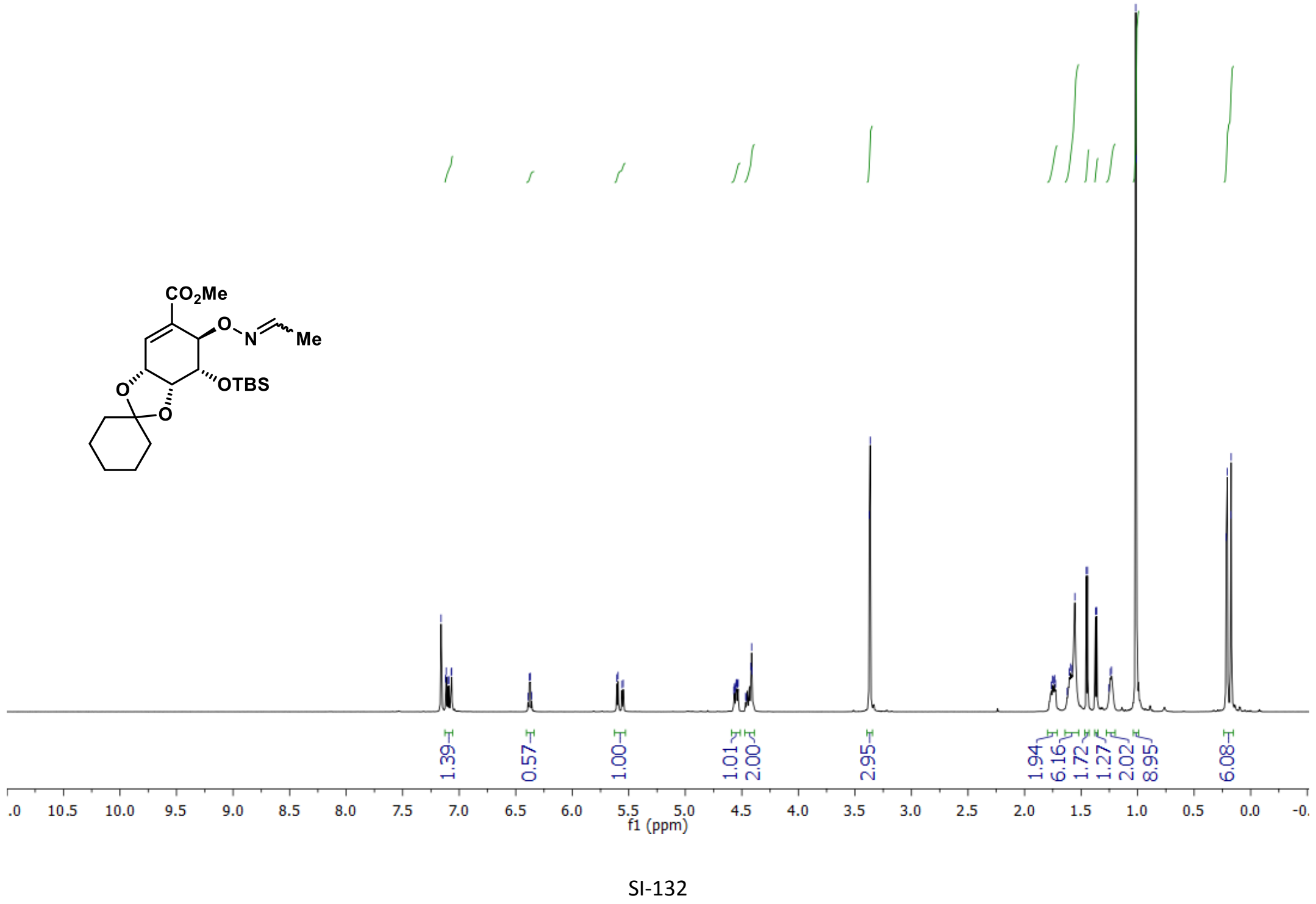




\section{Compound des-TMS-5 ${ }^{13} \mathrm{C}$ NMR}

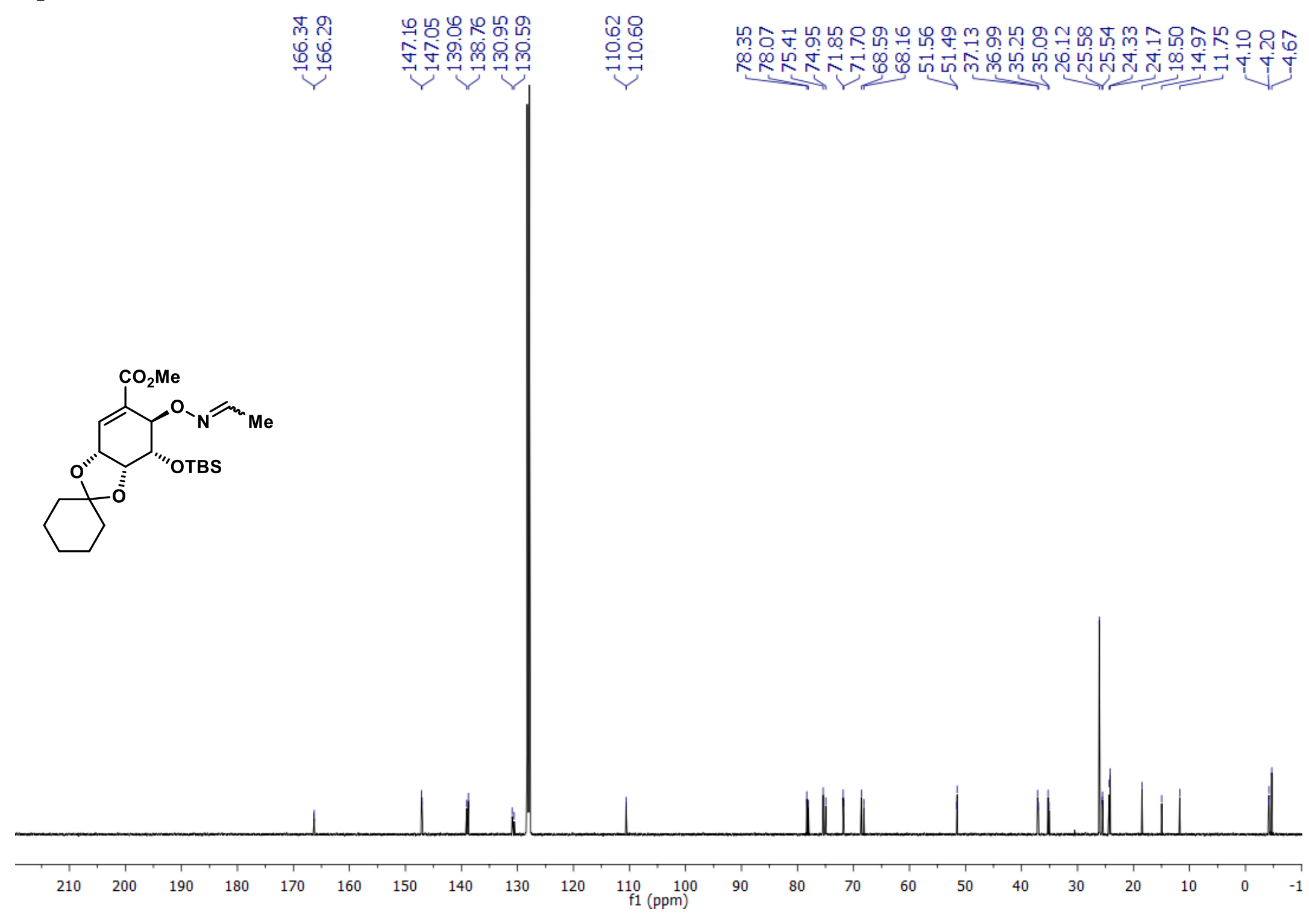




\section{Compound $18{ }^{1} \mathrm{H}$ NMR}

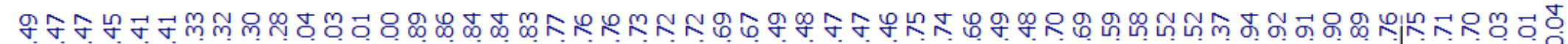

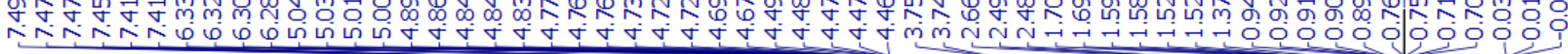
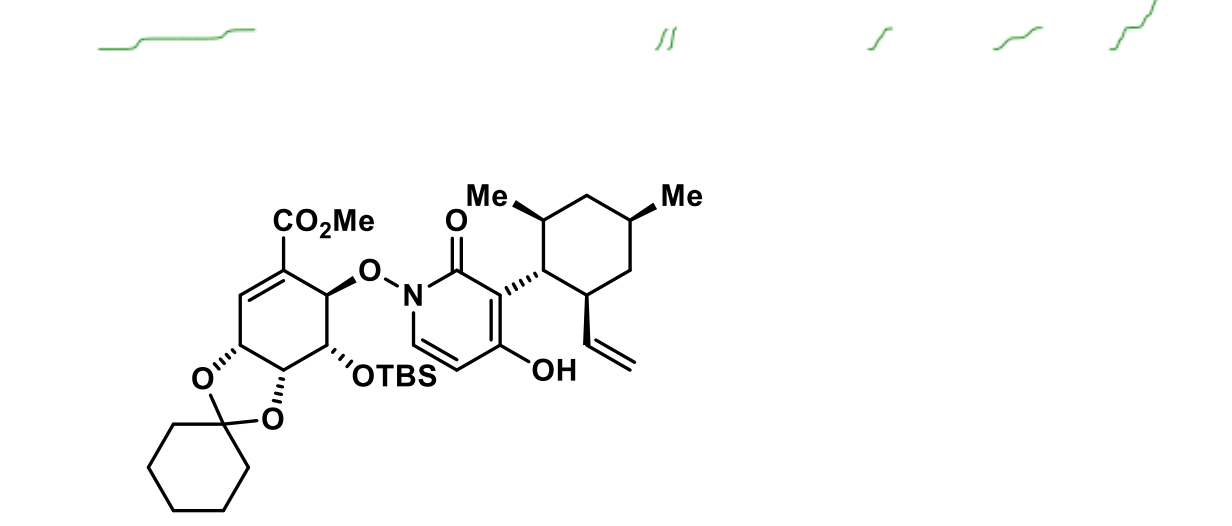


\section{Compound $18{ }^{13} \mathrm{C}$ NMR}

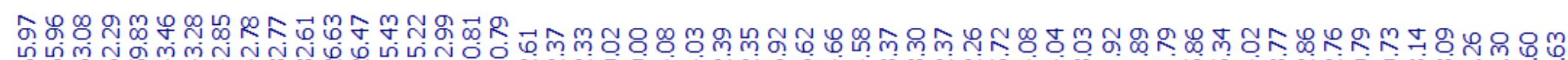

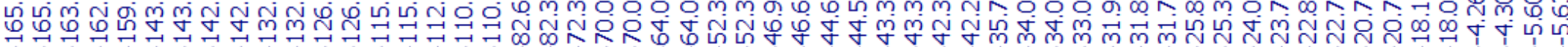

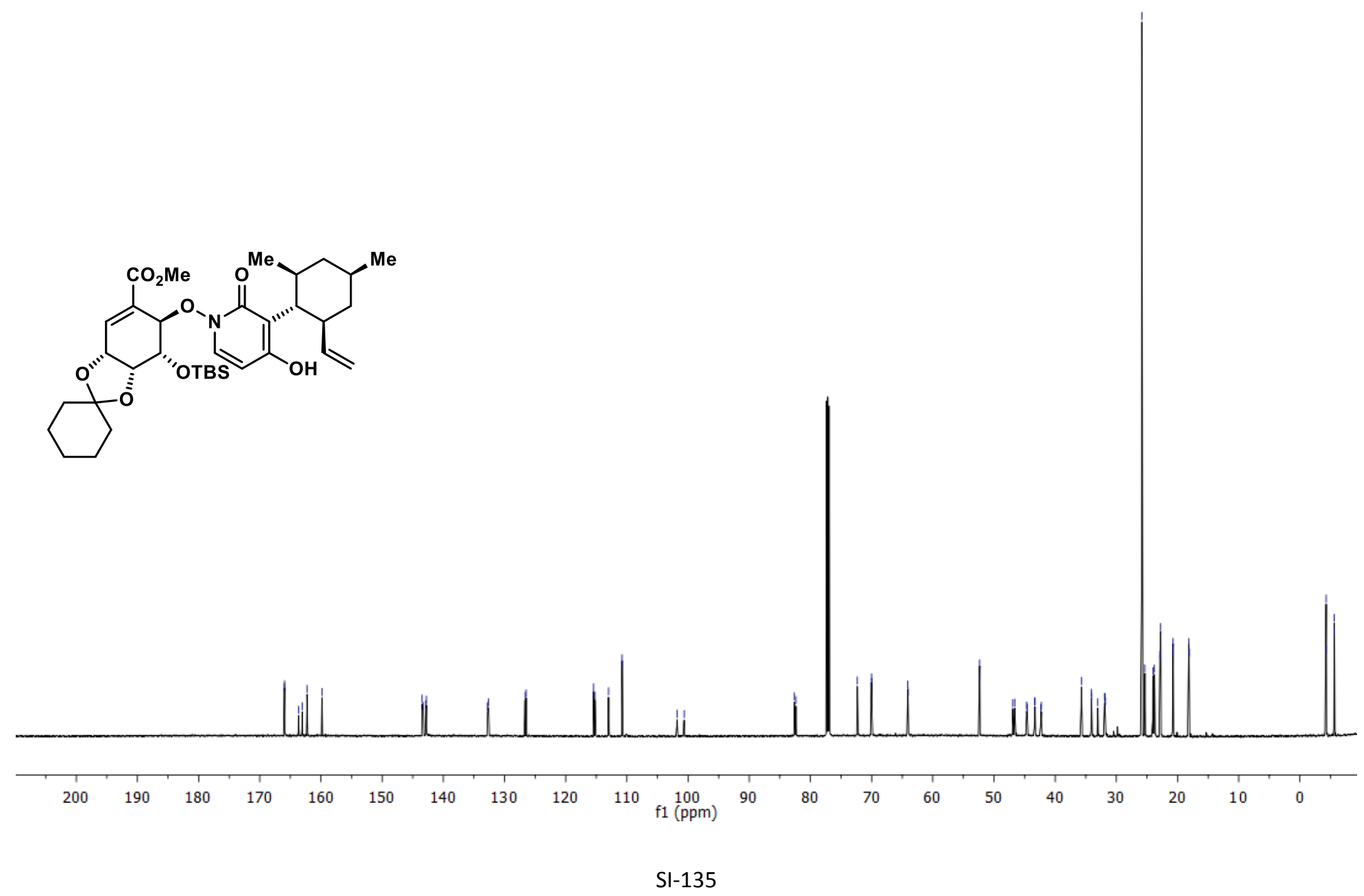




\section{Compound $18{ }^{1} \mathrm{H}$ NMR}

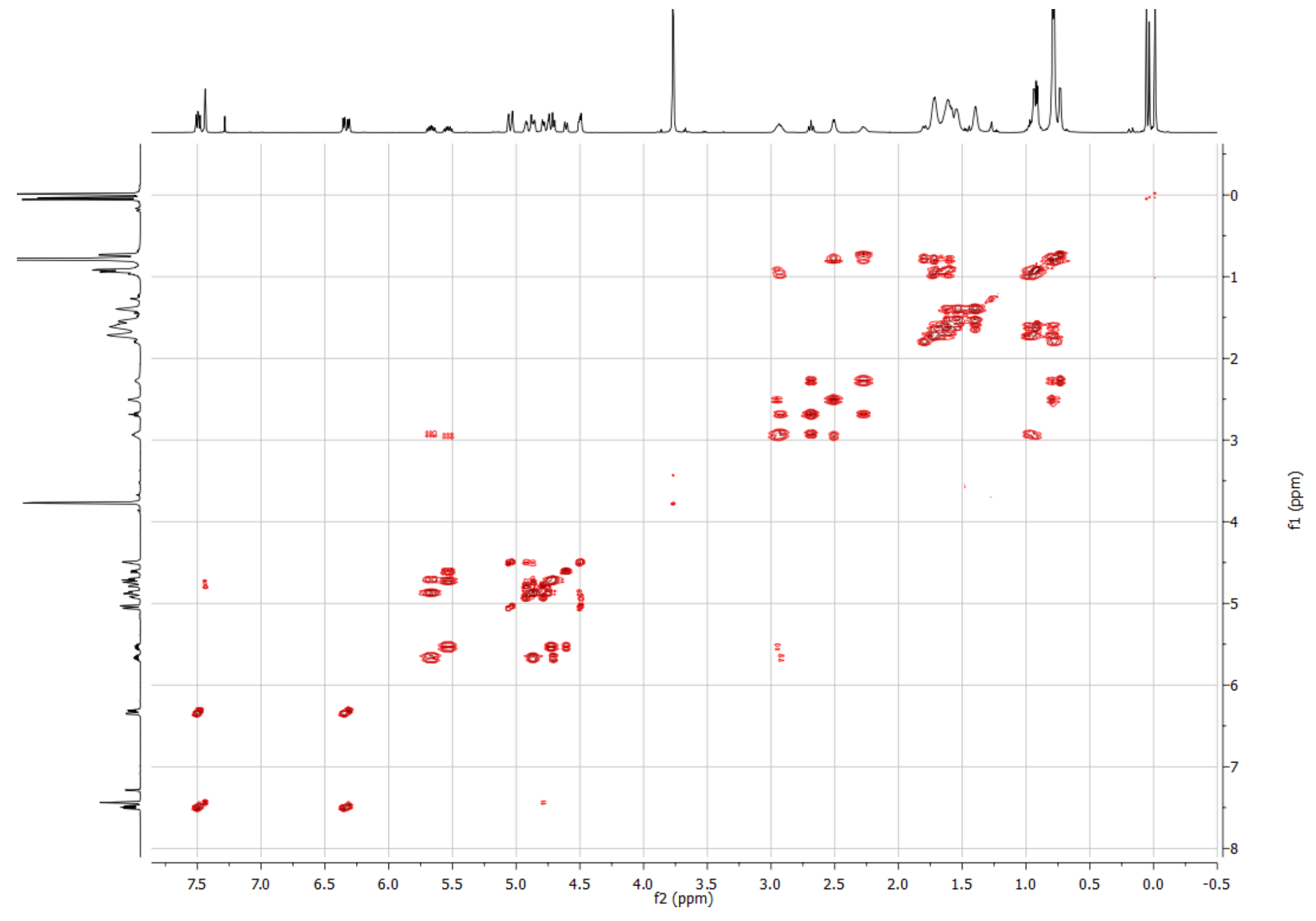




\section{Compound $18{ }^{13} \mathrm{C}$ NMR}

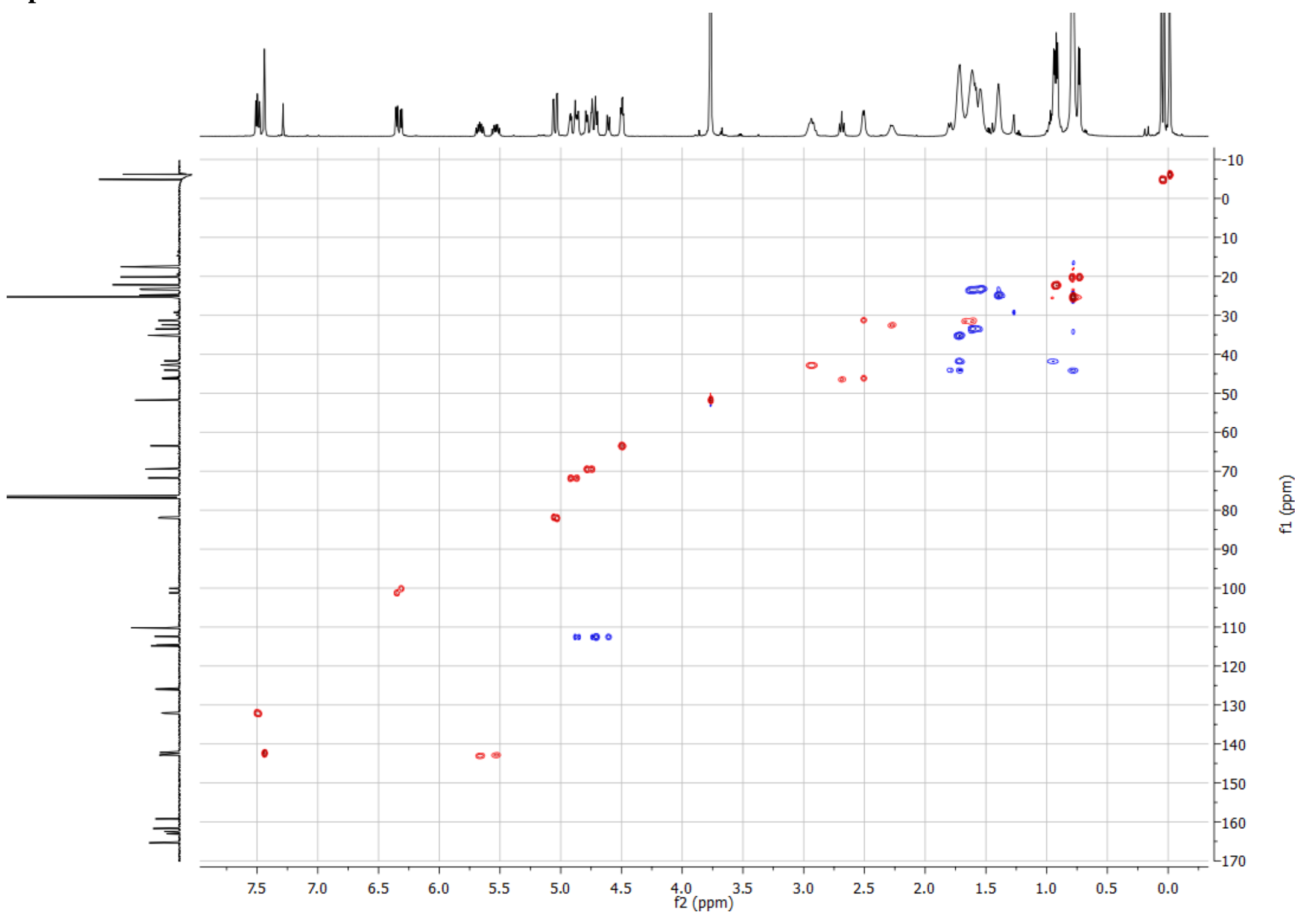




\section{Compound $1{ }^{1} \mathrm{H}$ NMR}

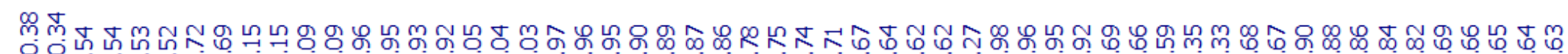

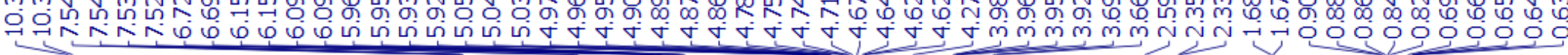

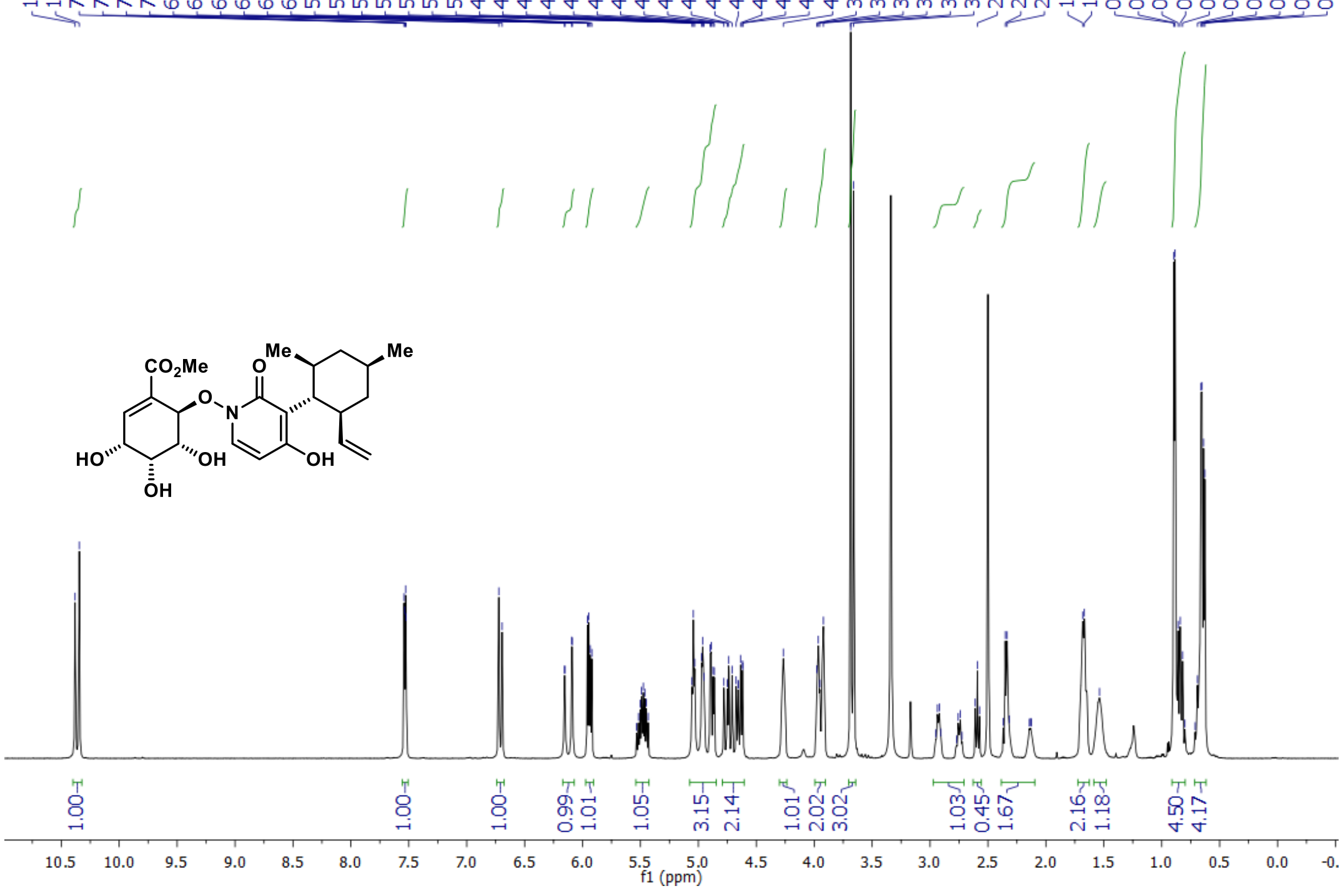




\section{Compound $1{ }^{13} \mathrm{C}$ NMR}

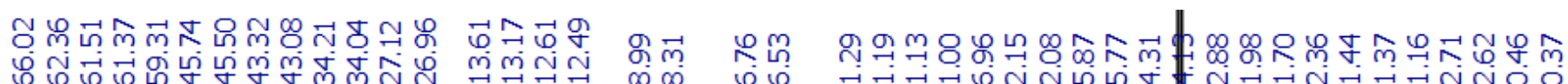

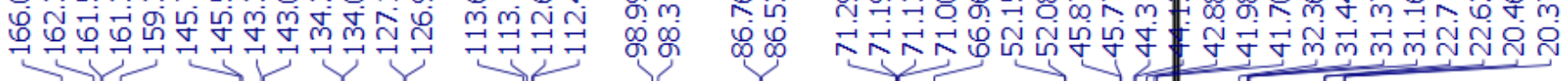
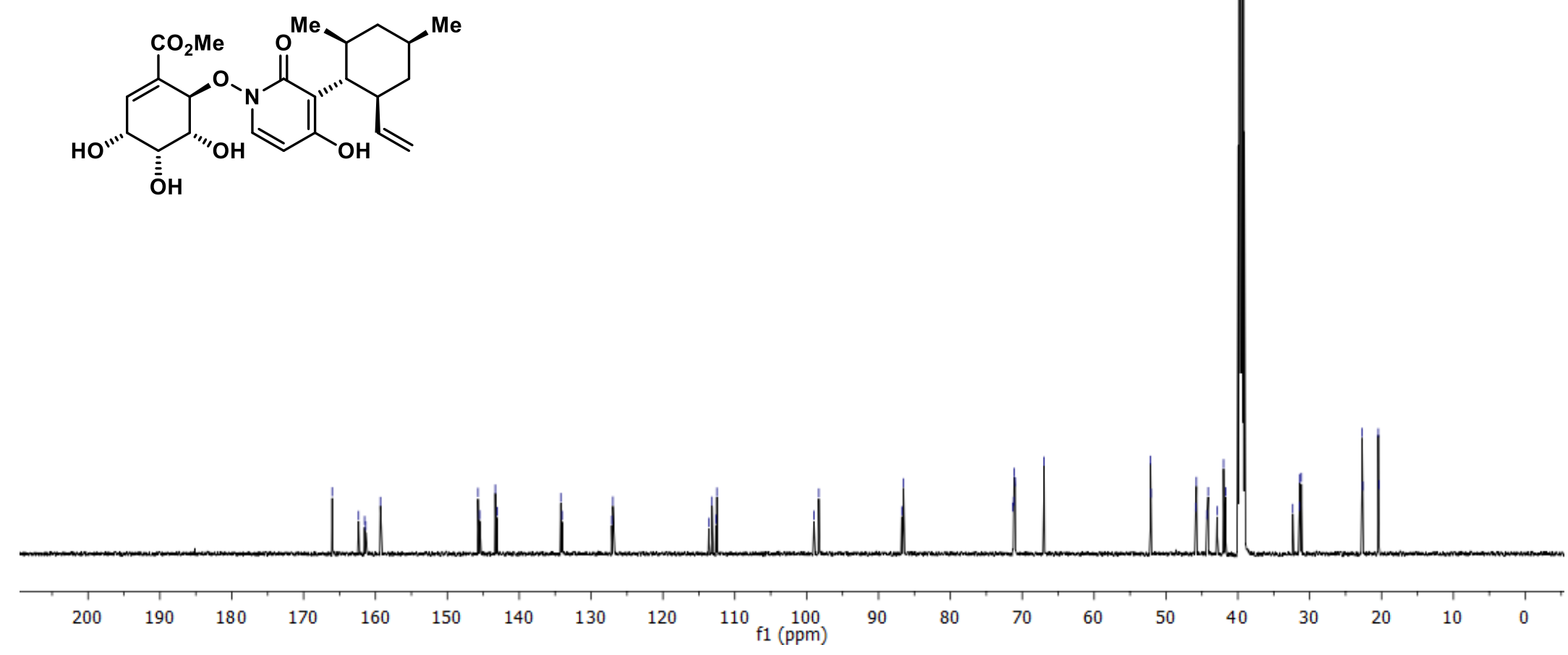


\section{Compound 1 HSQC}

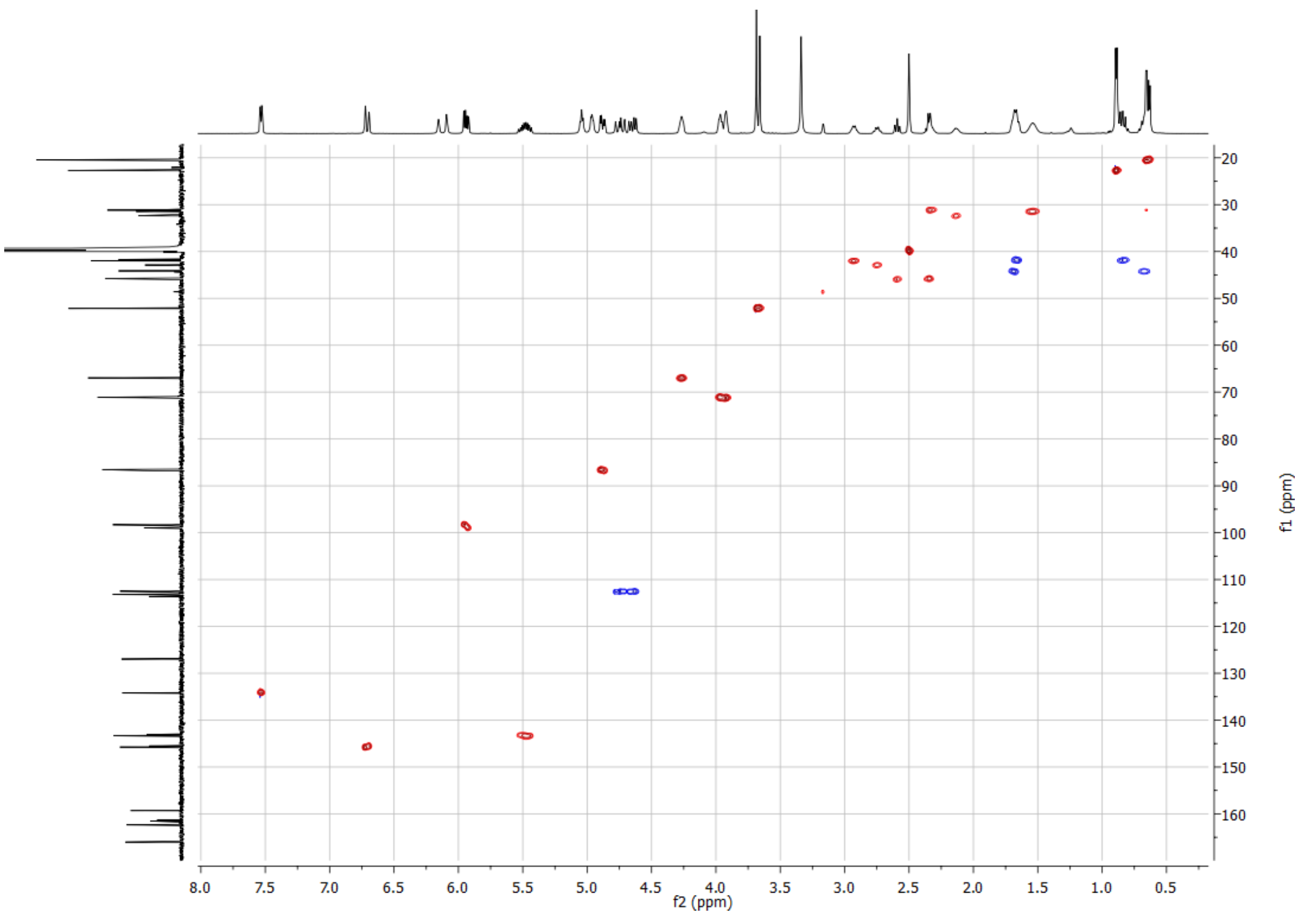




\section{Compound 10a ${ }^{1} \mathrm{H}$ NMR}

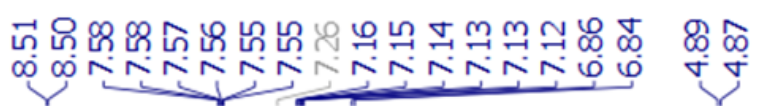

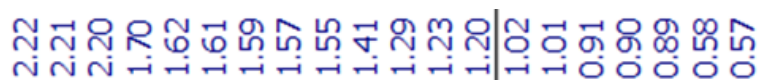
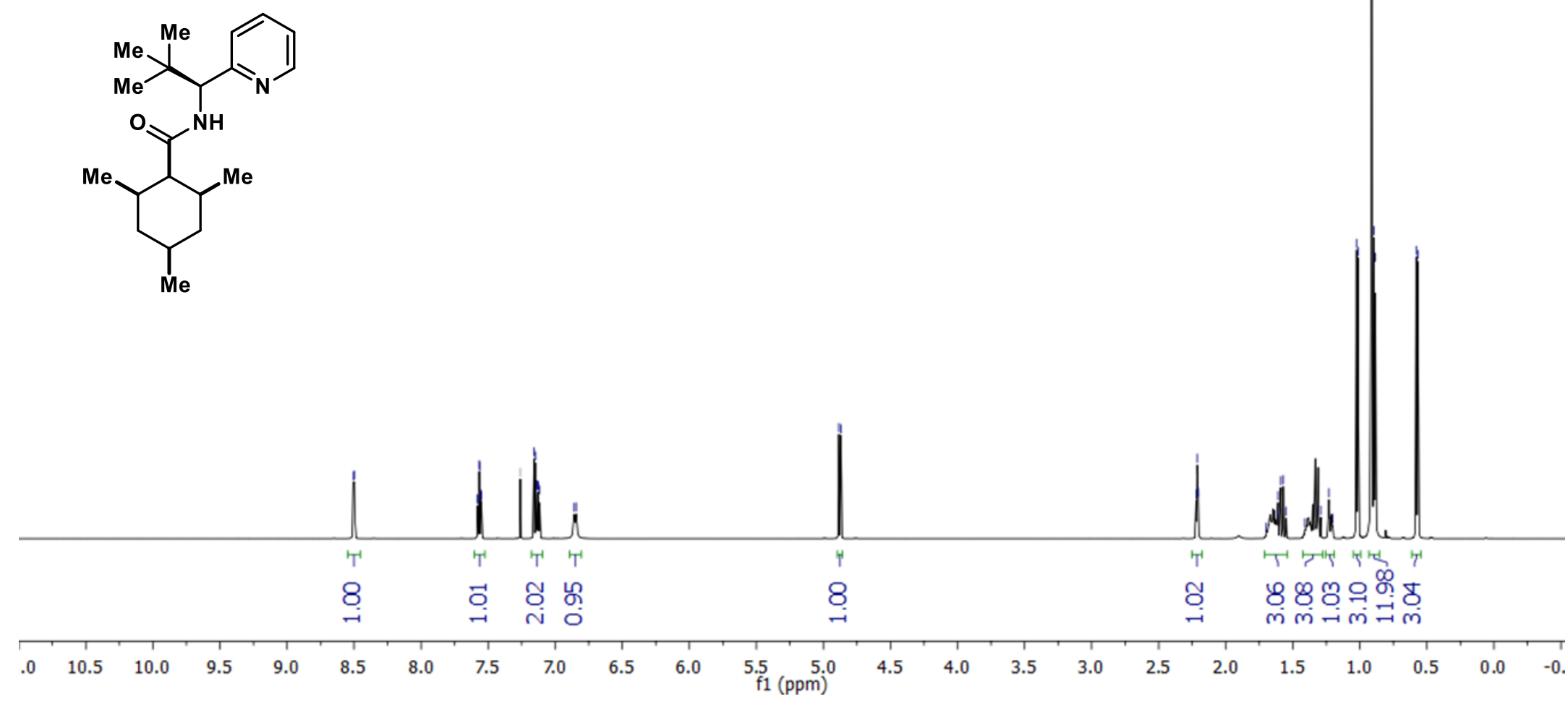

SI-141 


\section{Compound 10a ${ }^{13} \mathrm{C}$ NMR}

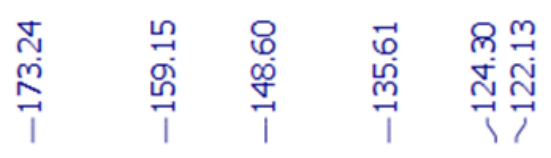

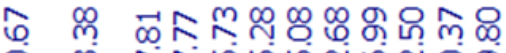

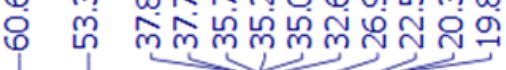

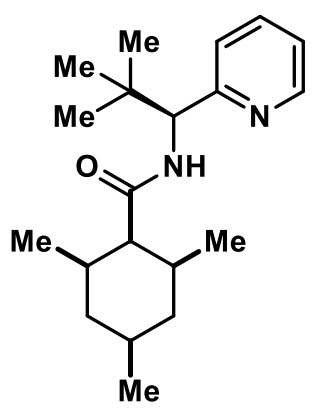

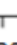

200

170

130

${ }_{\mathrm{f} 1}^{110}(\mathrm{ppm})$

90

80

60

50

30

20

SI-142 


\section{Compound 10b ${ }^{1} \mathrm{H}$ NMR}

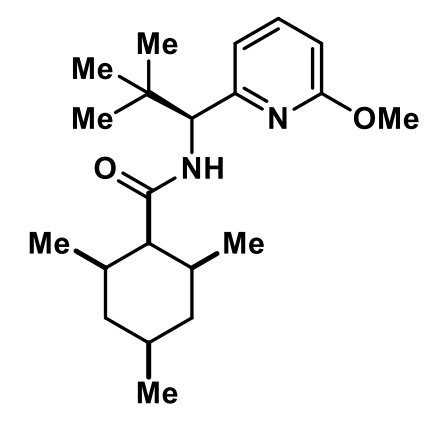

SI-143 


\section{Compound 10b ${ }^{13} \mathrm{C}$ NMR}

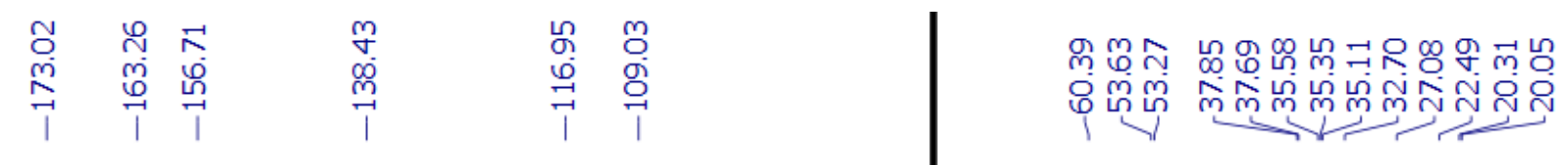

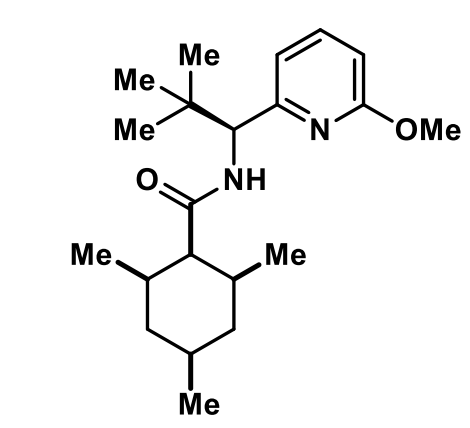

(n)

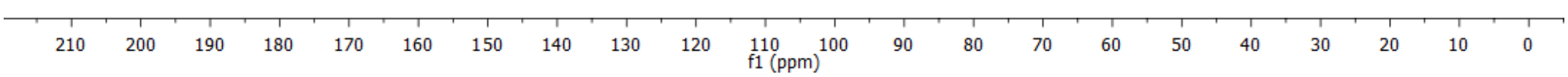

SI-144 


\section{Compound 10c ${ }^{1} \mathrm{H}$ NMR}

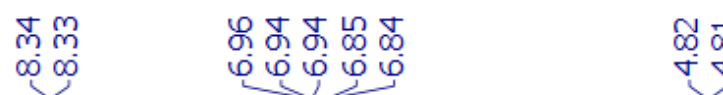

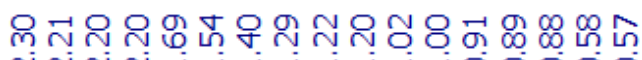
$\varphi \dot{\varphi} \varphi \dot{0}$
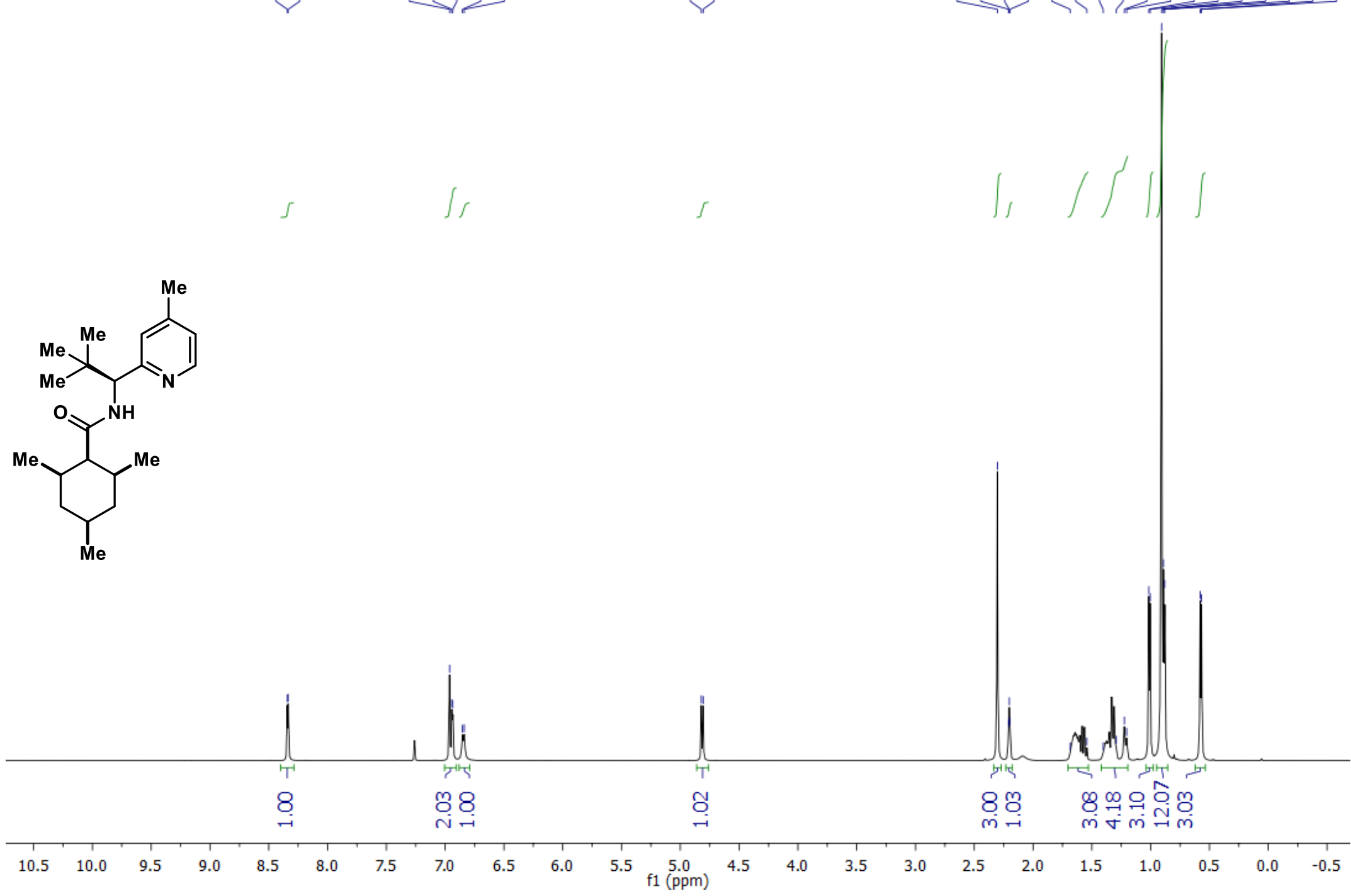


\section{Compound $10 \mathrm{c}^{13} \mathrm{C}$ NMR}

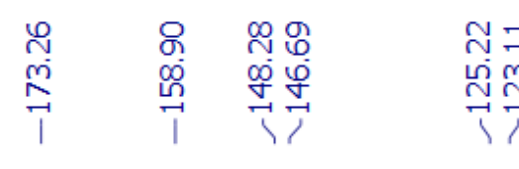

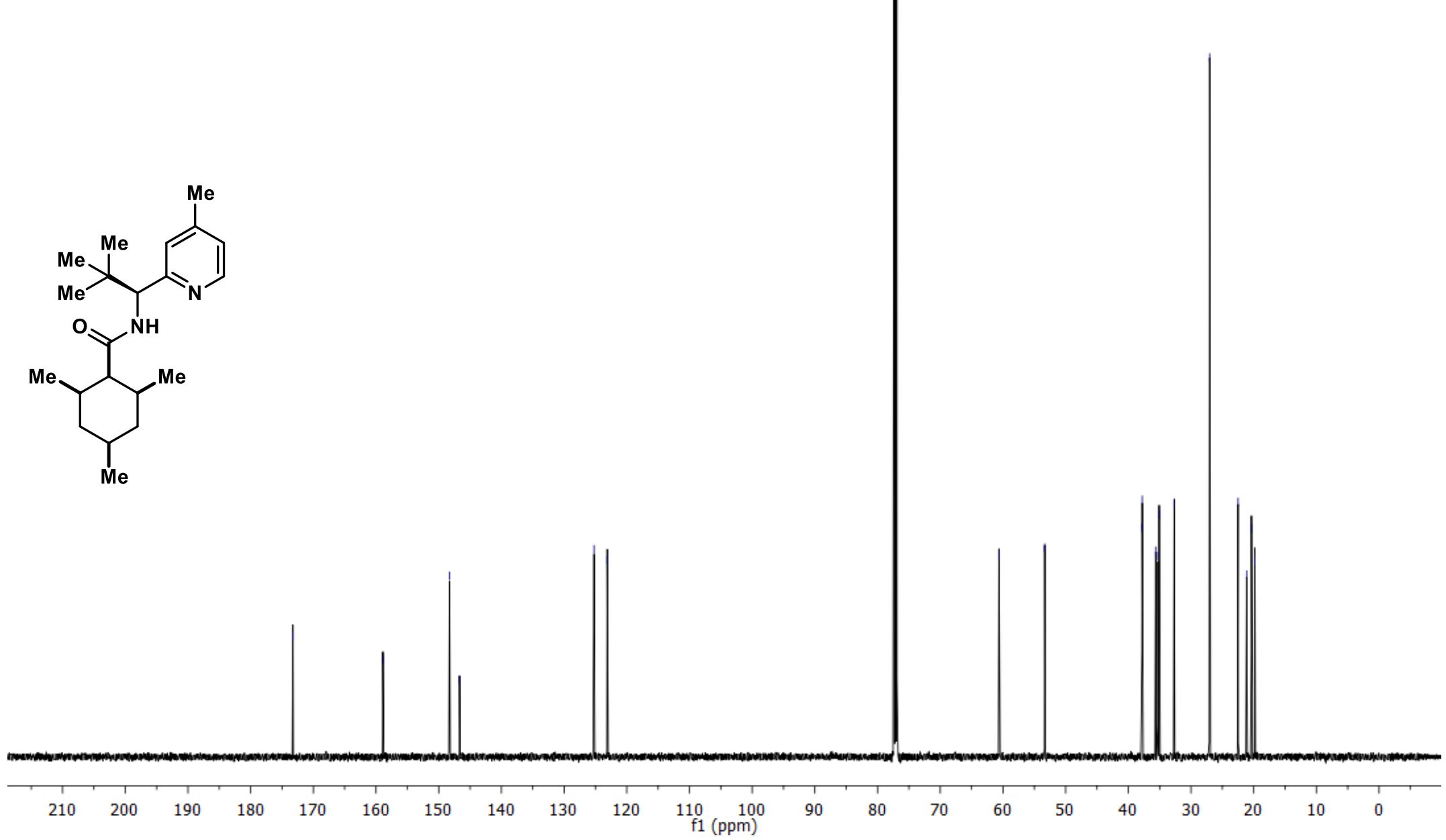




\section{Compound 10e ${ }^{1} \mathrm{H}$ NMR}

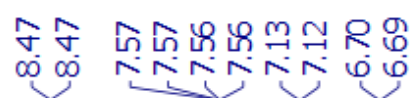
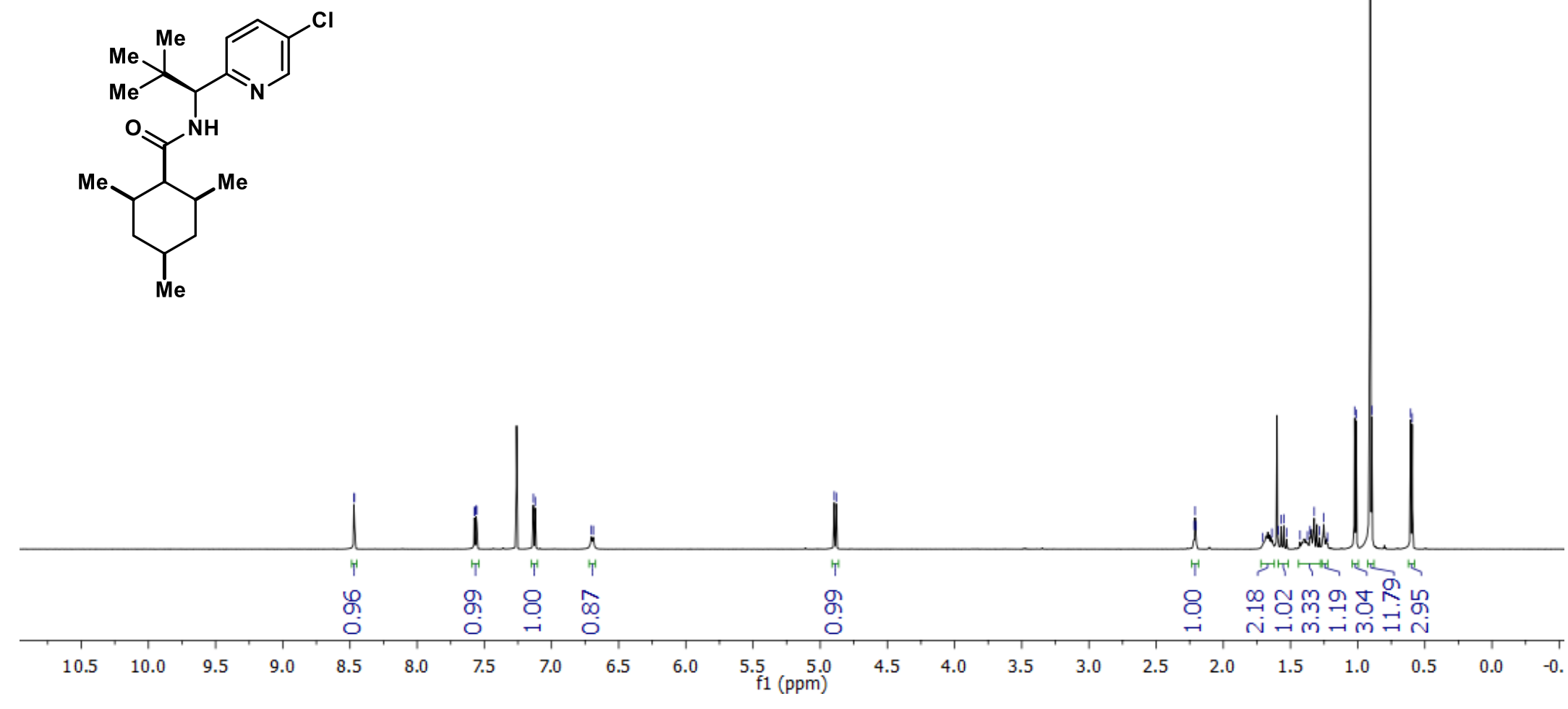


\section{Compound 10e ${ }^{13} \mathrm{C}$ NMR}
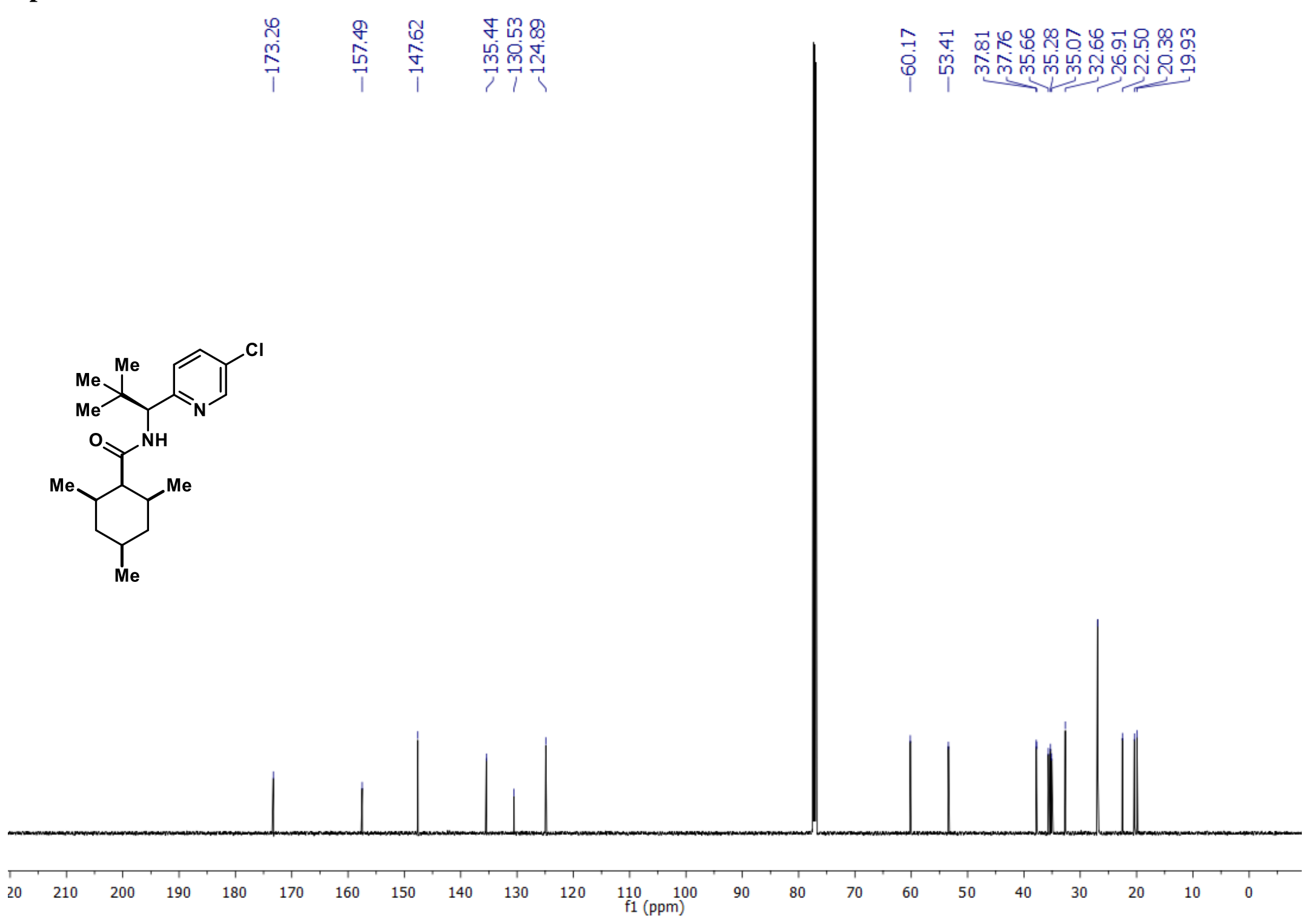


\section{Compound 10f ${ }^{1} \mathrm{H}$ NMR}

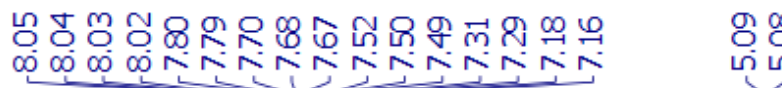

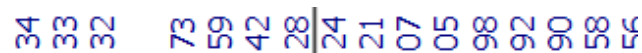

NNN Thitititiolo

$\iiint \iiint$

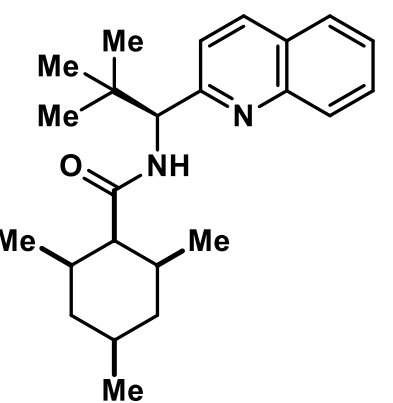




\section{Compound 10f ${ }^{13} \mathrm{C}$ NMR}

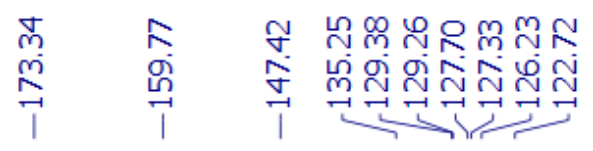

J $\quad$ 寸

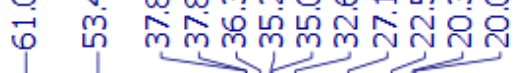

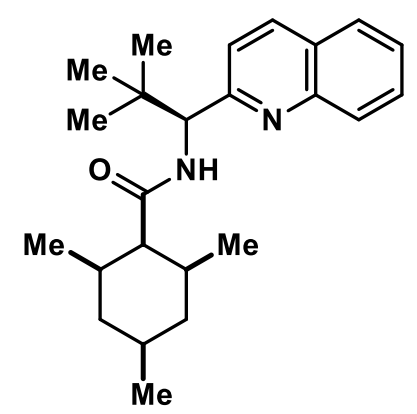

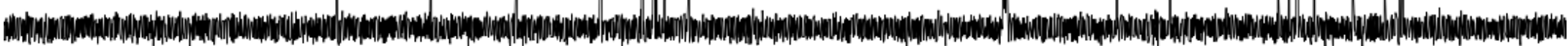

$\begin{array}{llllllllll}20 & 210 & 200 & 190 & 180 & 170 & 160 & 150 & 140 & 130\end{array}$

$\mathrm{f} 1(\mathrm{ppm}) \mathrm{100}$

$80 \quad 70$

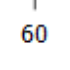

$50 \quad 40$

30

SI-150 


\section{Compound SI-4 ${ }^{1} \mathrm{H}$ NMR}

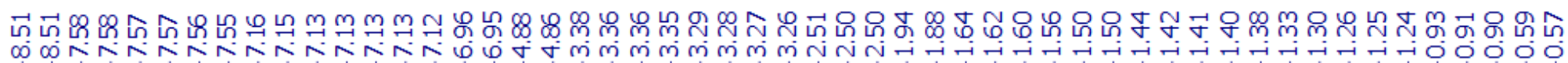
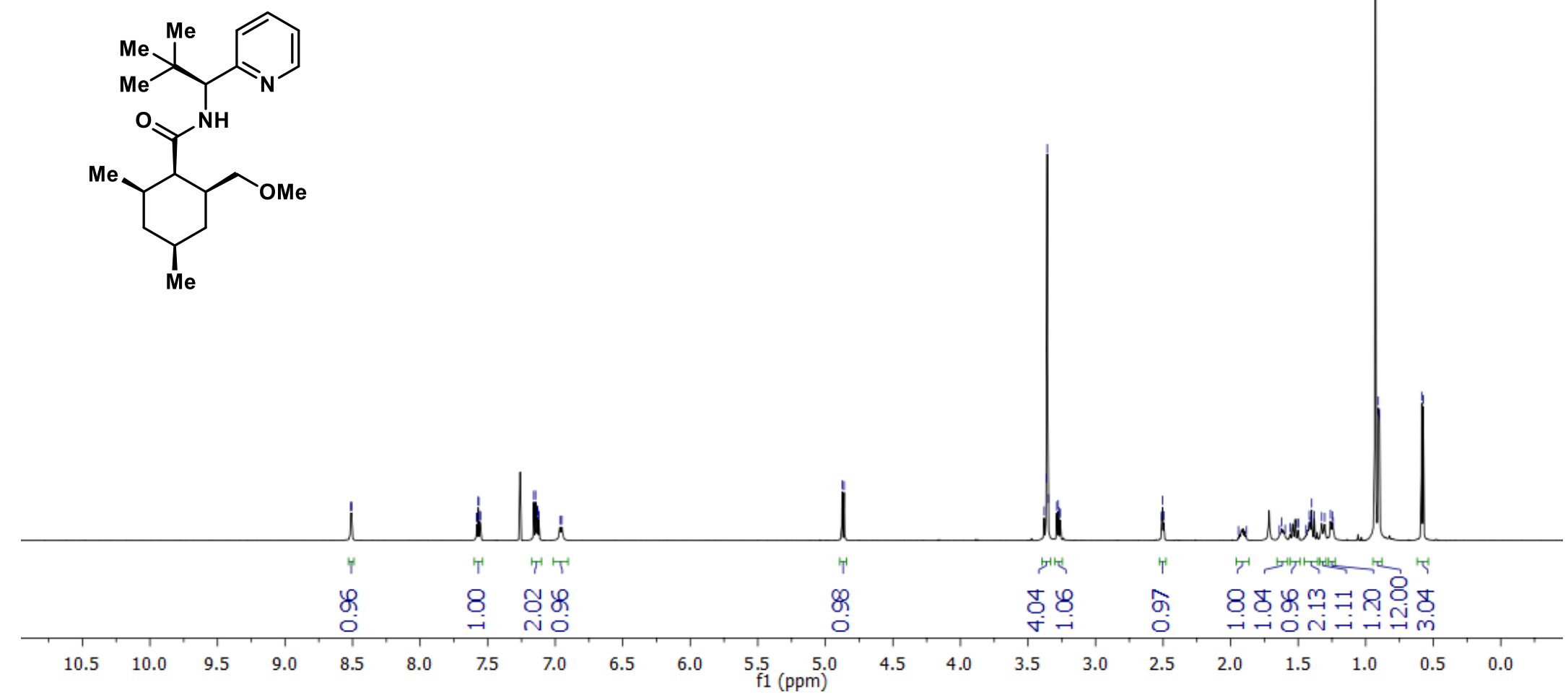


\section{Compound SI-4 ${ }^{13} \mathrm{C}$ NMR}

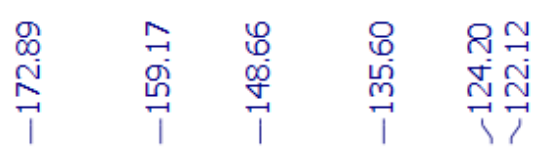

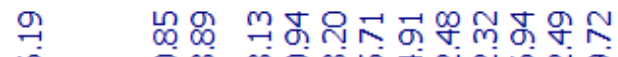

잉

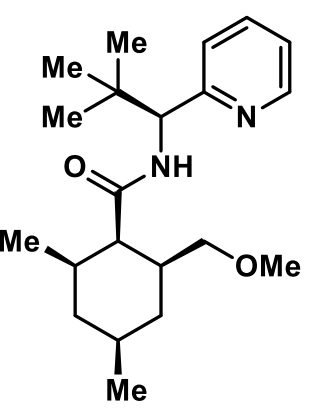

$\begin{array}{llllllllll}200 & 190 & 180 & 170 & 160 & 150 & 140 & 130 & 120 & \begin{array}{l}110 \\ \mathrm{f} 1(\mathrm{ppm})\end{array}\end{array}$

90

80

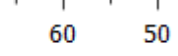




\section{Compound SI-5 ${ }^{1} \mathrm{H}$ NMR}

있

$\infty$

హิ फ़
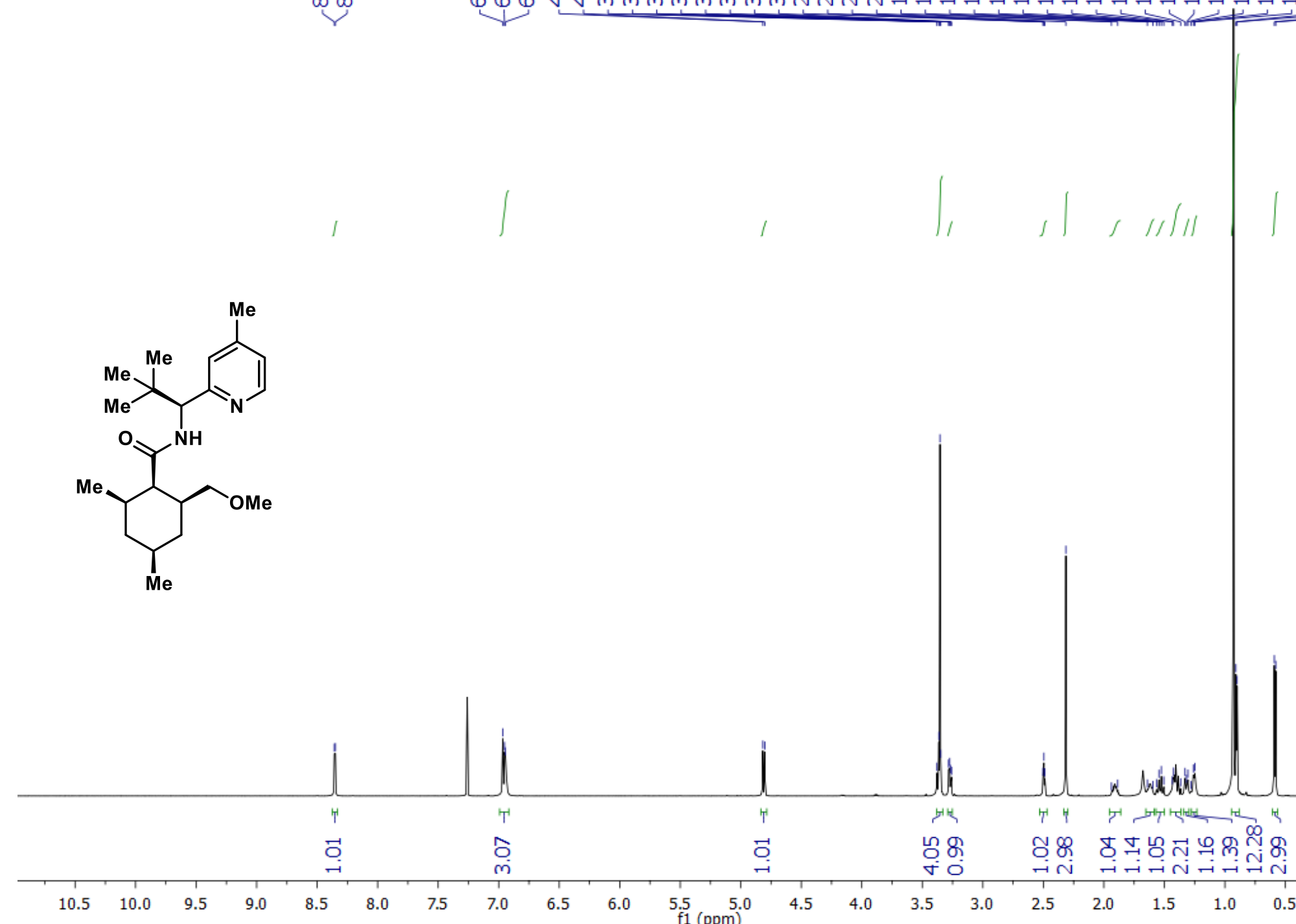

$\begin{array}{lllllllllll}10.5 & 10.0 & 9.5 & 9.0 & 8.5 & 8.0 & 7.5 & 7.0 & 6.5 & 6.0 & 5.5 \\ & & & & & & & & & & \\ \text { f1 (ppm) }\end{array}$ 


\section{Compound SI-5 ${ }^{13} \mathrm{C}$ NMR}

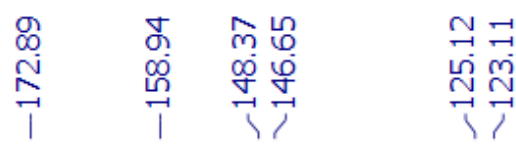

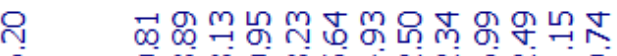

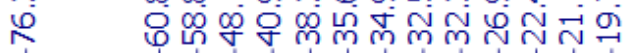
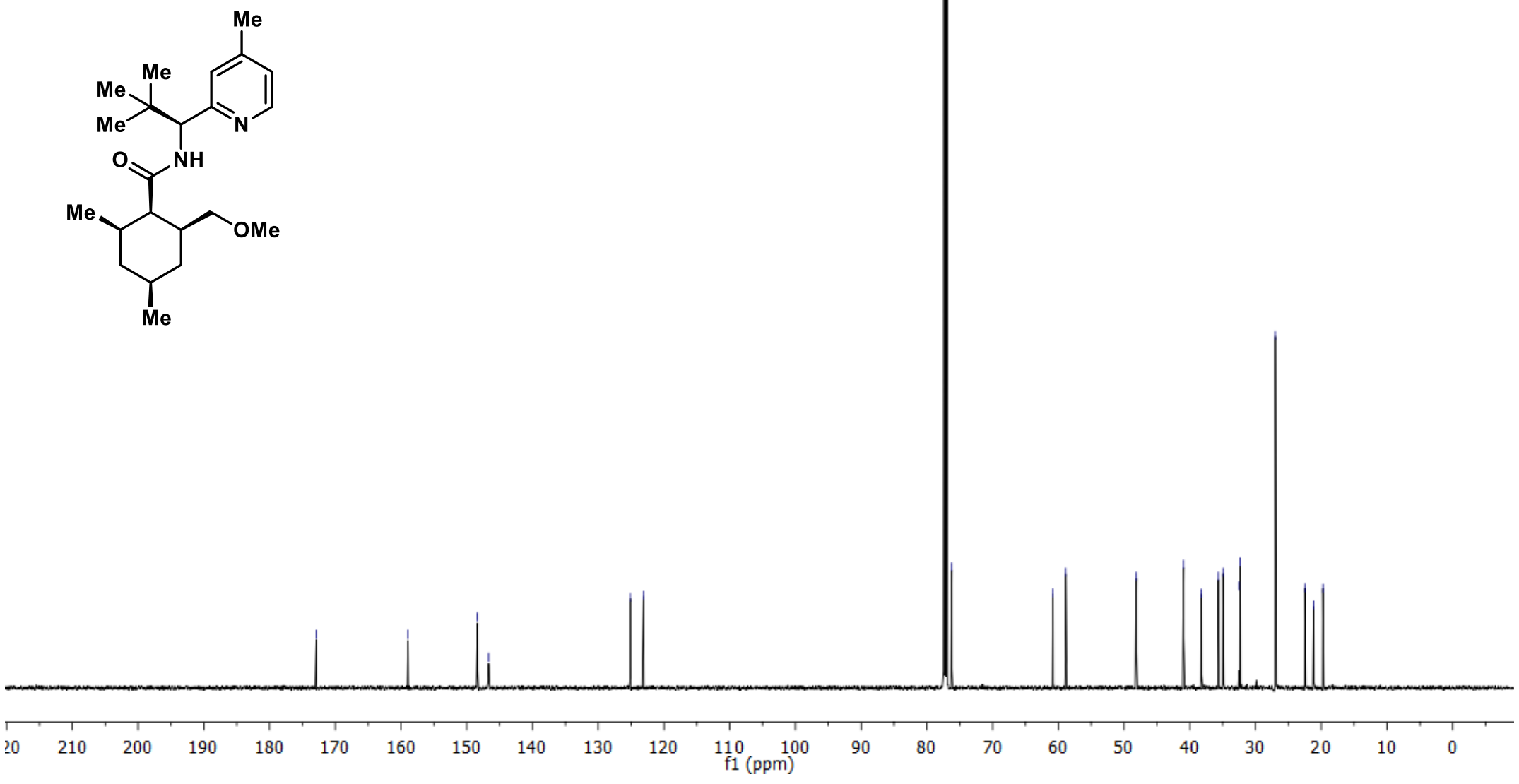


\section{Compound SI-7 ${ }^{1}$ H NMR}

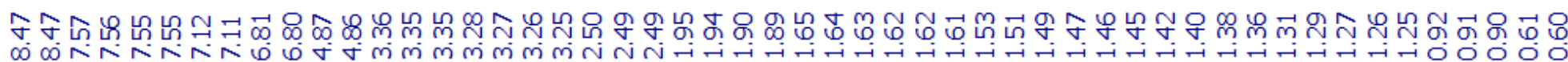

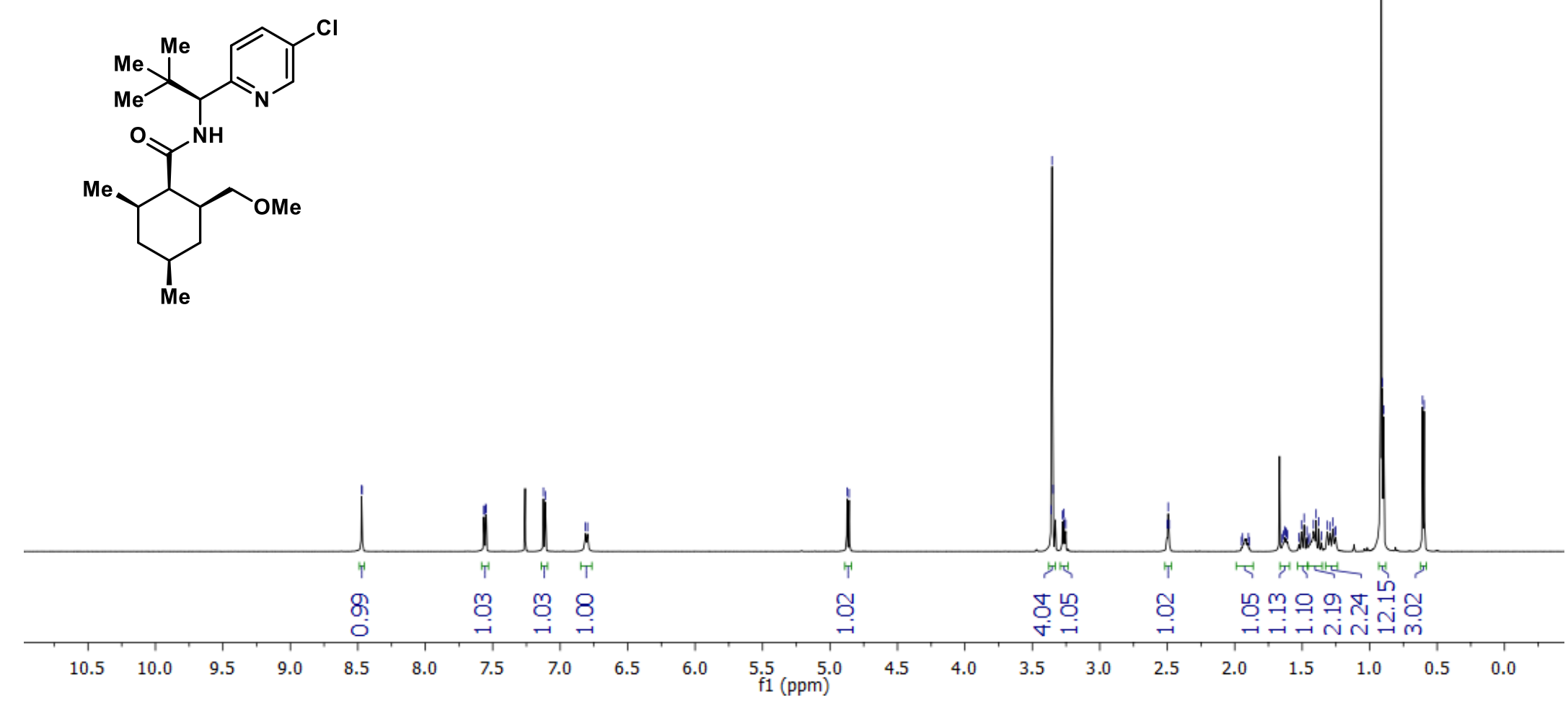




\section{Compound SI-7 ${ }^{13} \mathrm{C}$ NMR}

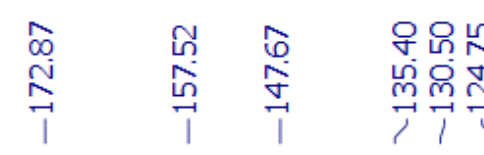

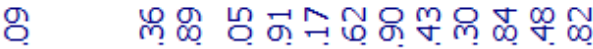

ڤ.
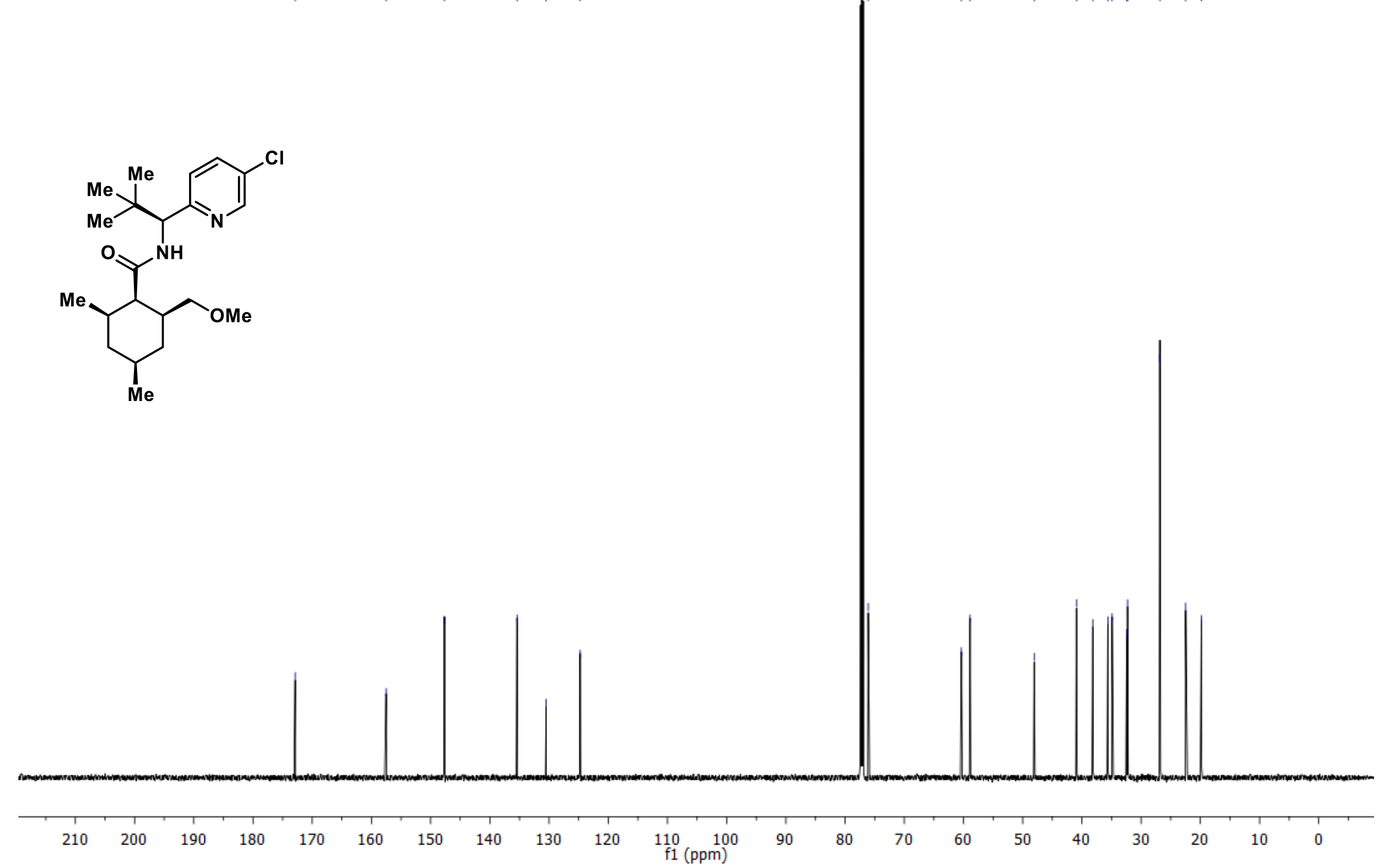


\section{Compound SI-8 ${ }^{1} \mathrm{H}$ NMR}

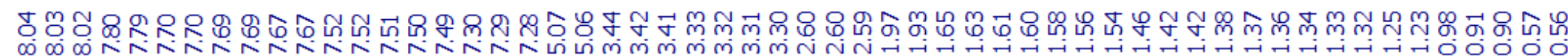
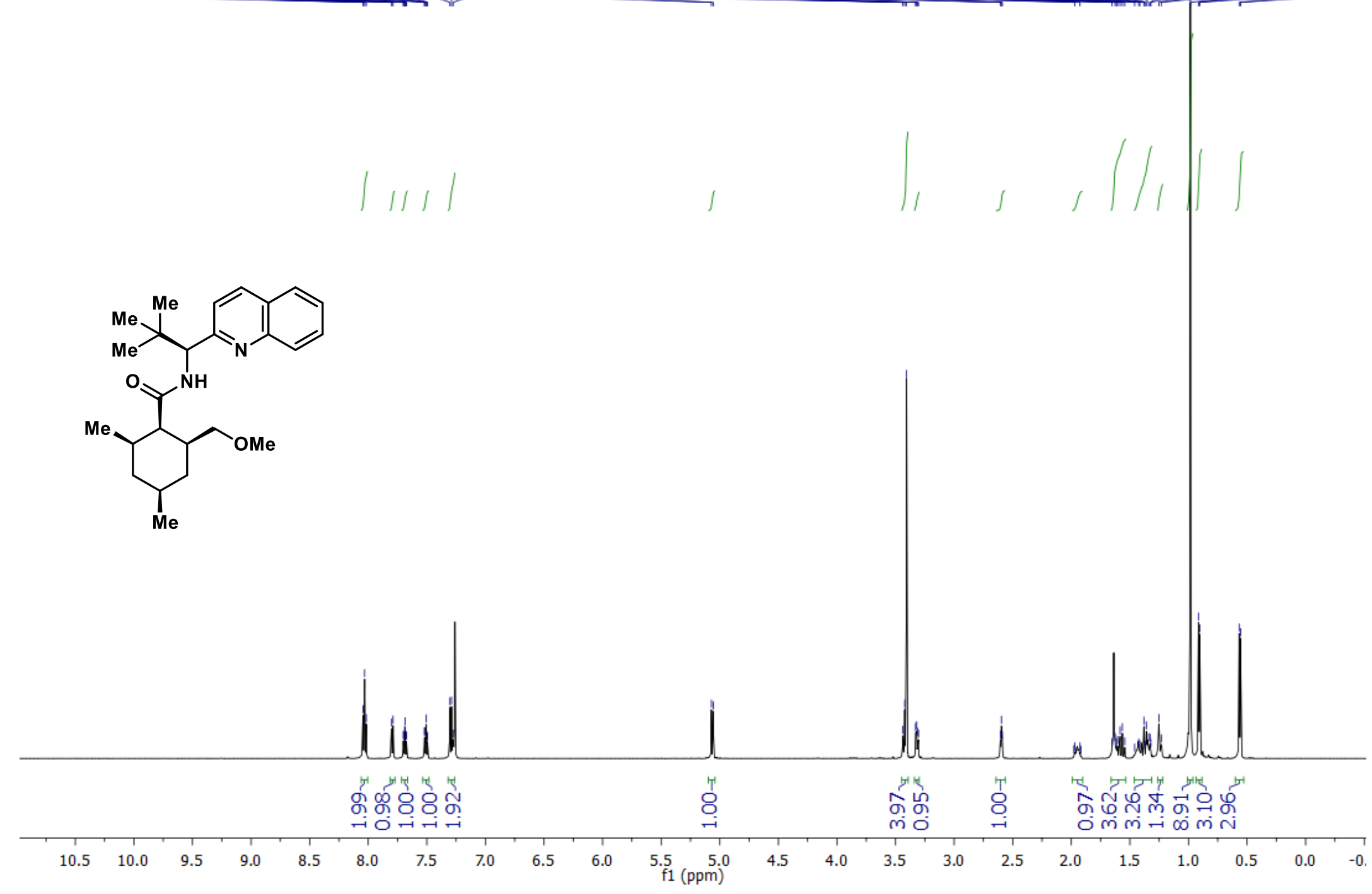


\section{Compound SI-8 ${ }^{13} \mathrm{C}$ NMR}
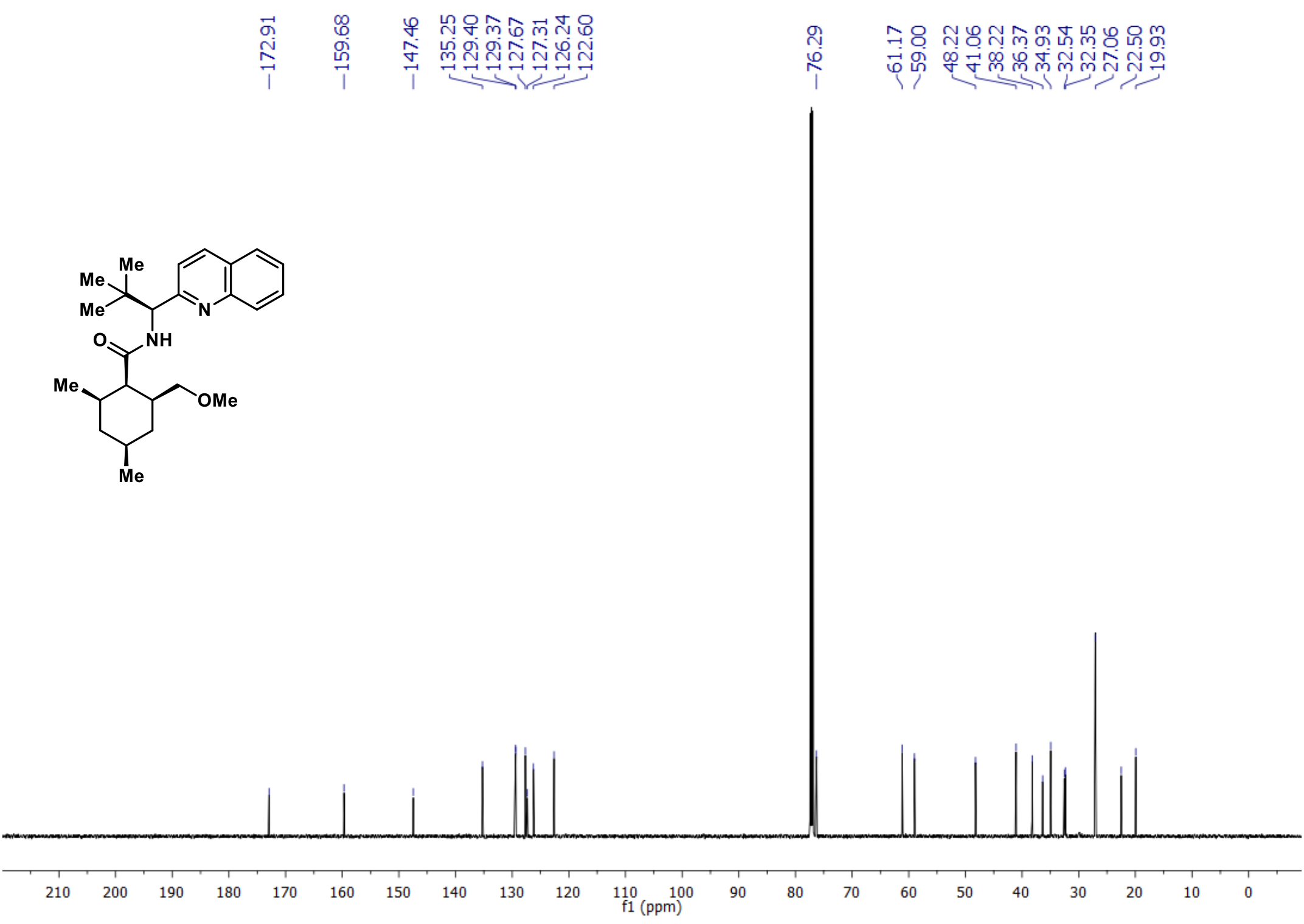


\section{Compound SI-2 ${ }^{1} \mathrm{H}$ NMR}

न

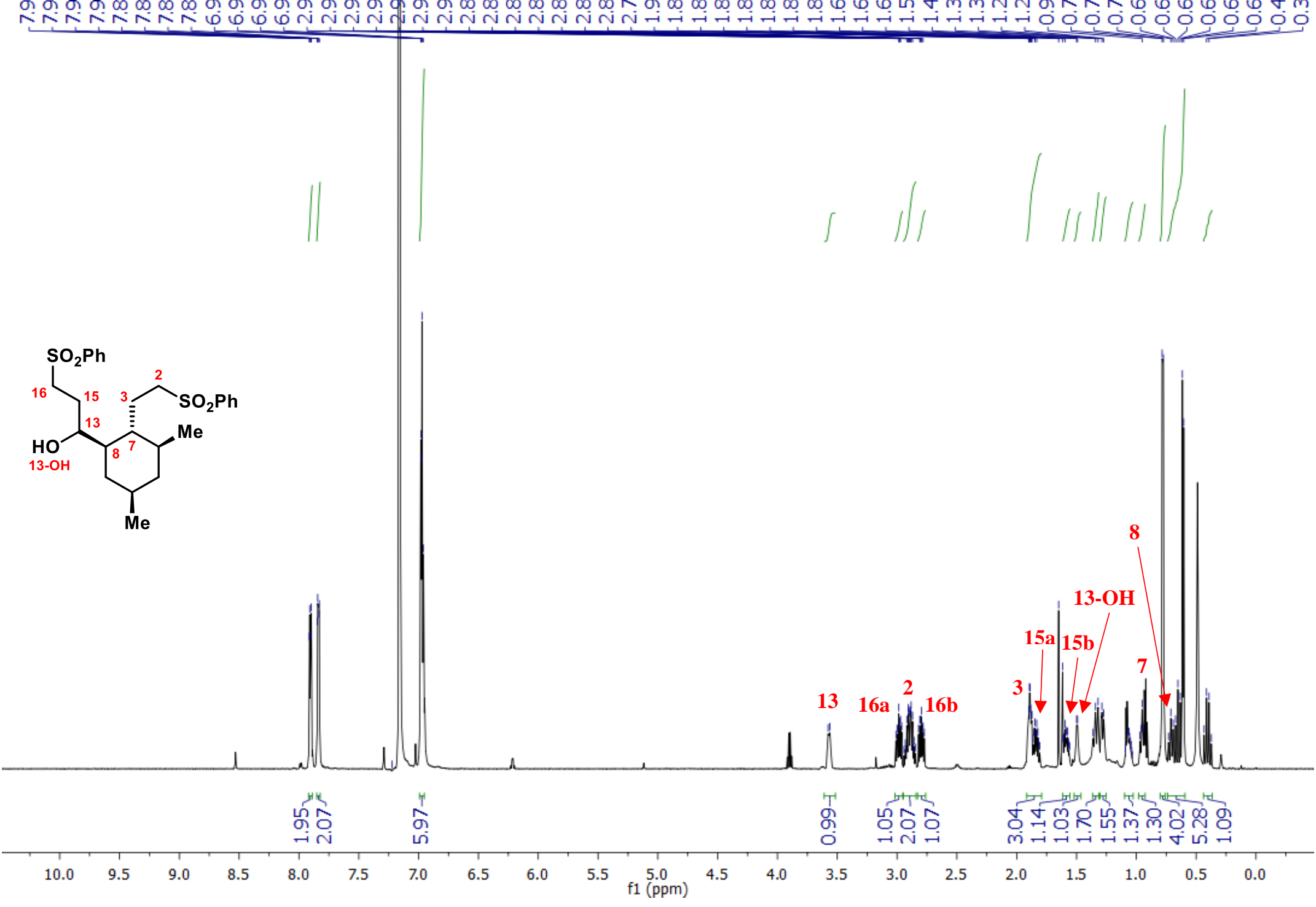




\section{Compound SI-2 ${ }^{13} \mathrm{C}$ NMR}
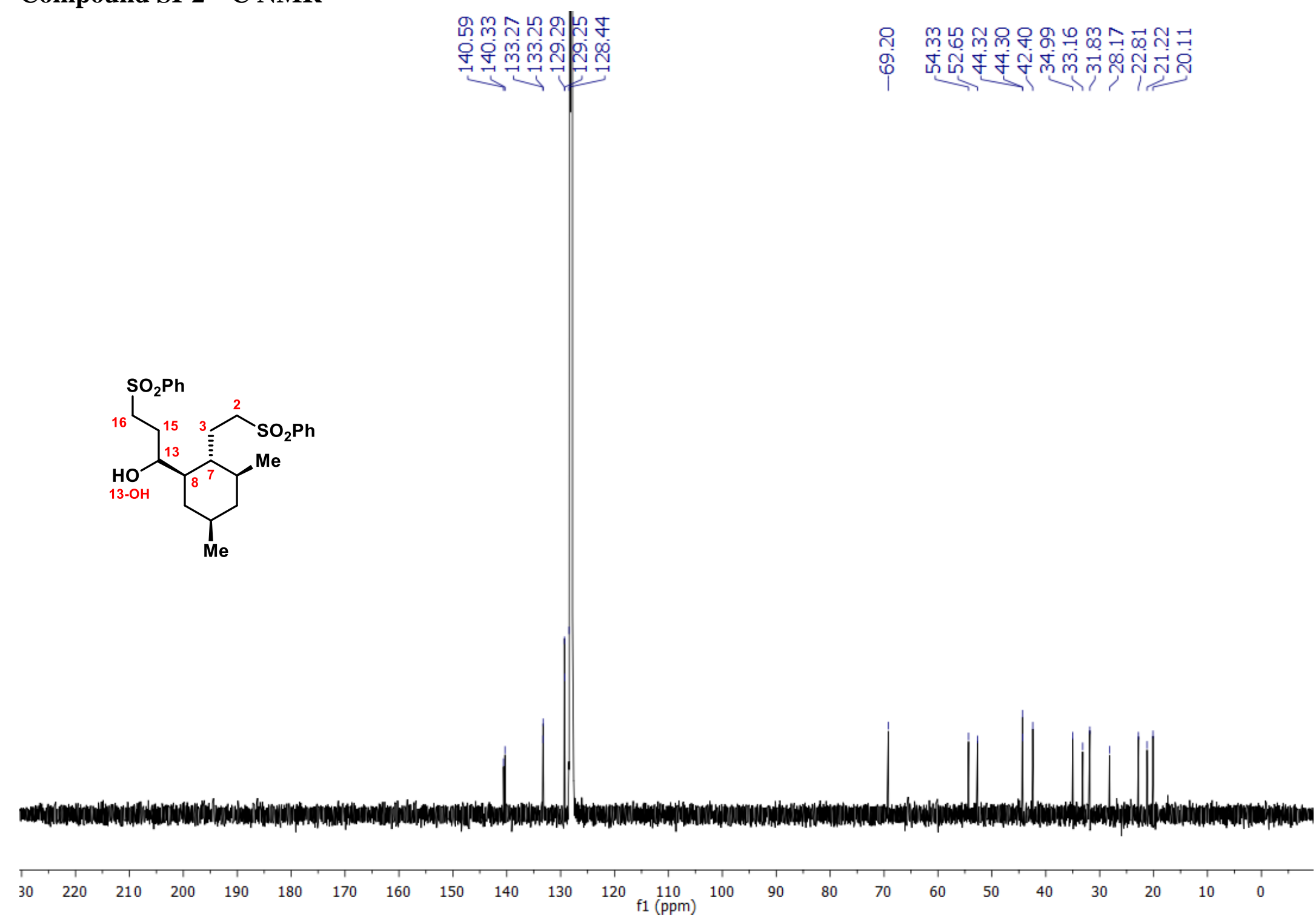

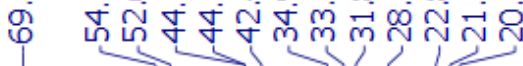




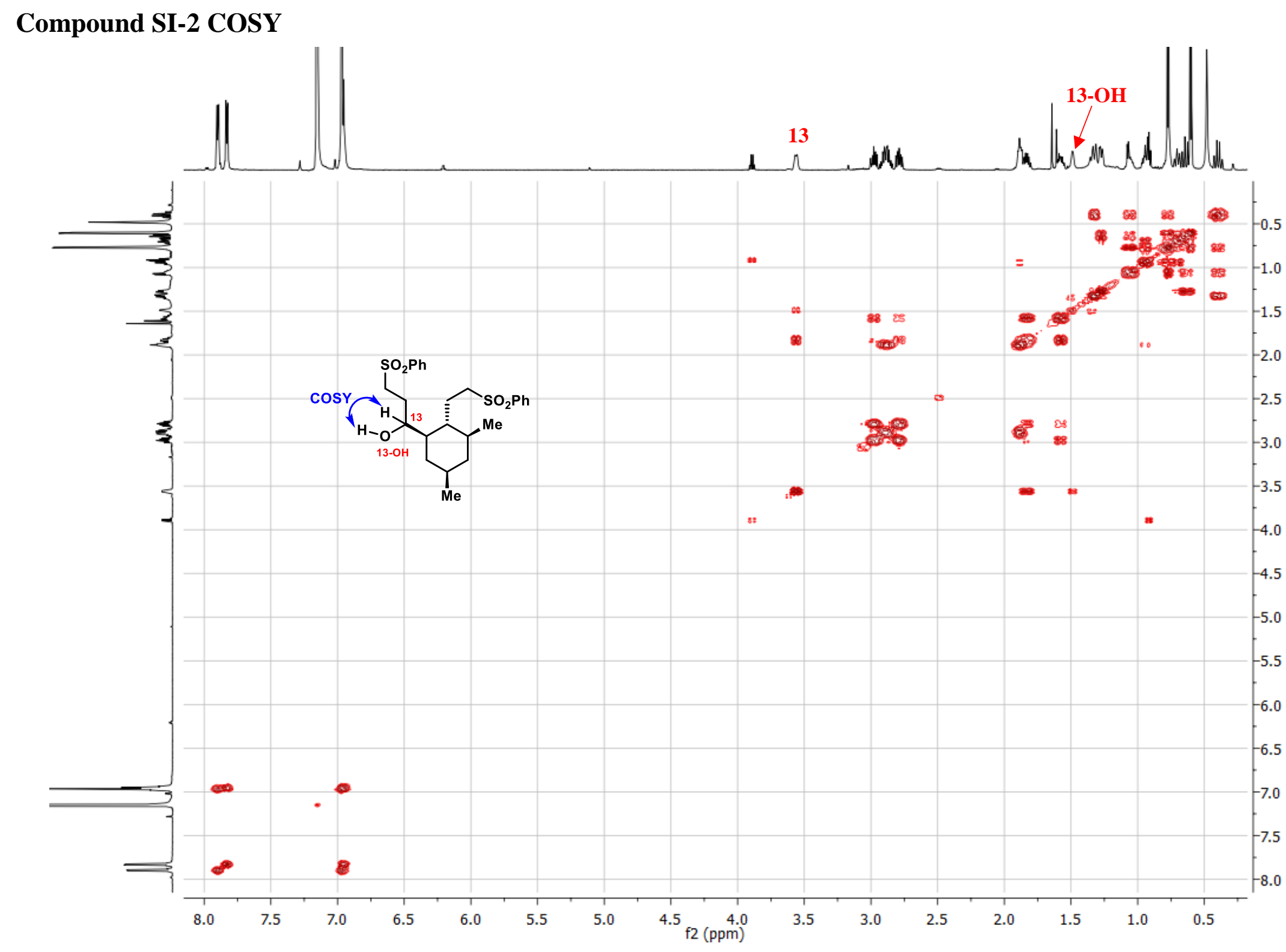




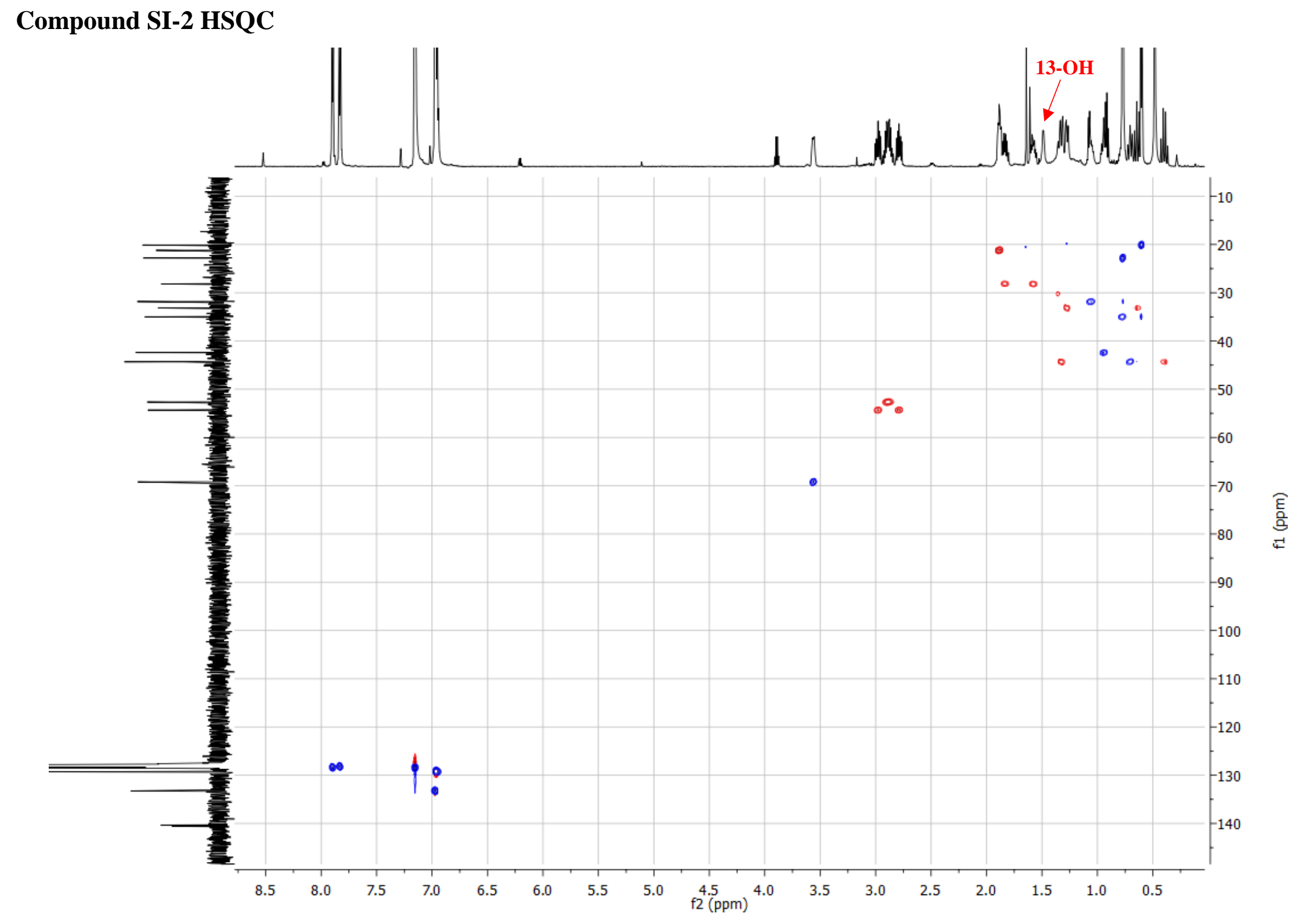

SI-162 